\title{
Zielorientierungen und schulisches Lernen am Grundschulübergang
}

\author{
Dissertation \\ zur Erlangung des Doktorgrades \\ der Sozialwissenschaftlichen Fakultät \\ der Georg-August-Universität Göttingen
}

vorgelegt von

Isabell Paulick

geboren in Altdöbern

Göttingen 2011 
Erstgutachter: $\quad$ Prof. Dr. Rainer Watermann

Zweitgutachter: $\quad$ Prof. Dr. Matthias Nückles

Tag der mündlichen Prüfung: 10.11.2011 


\section{Danke}

Diese Dissertation wurde durch ein Stipendium im Rahmen des Graduiertenkollegs „Passungsverhältnisse schulischen Lernens“ von der Deutschen Forschungsgemeinschaft gefördert. Für die finanzielle und materielle Unterstützung bedanke ich mich sehr. Die Grundlage dieser Dissertation bilden die Daten der TIMSS-Übergangsstudie, welche mir zur Analyse bereitgestellt wurden. In diesem Zusammenhang möchte ich mich rechtherzlich bei Herrn Prof. Dr. Rainer Watermann bedanken.

Mein besonderer Dank gilt meinen beiden Betreuern Herrn Prof. Dr. Rainer Watermann und Herrn Prof. Dr. Matthias Nückles. Herrn Watermann danke ich insbesondere für die wertvollen methodischen und inhaltlichen Hinweise und Anregungen während der gesamten Promotionszeit. Bei Herrn Nückles bedanke ich mich für die hilfreichen Anmerkungen und Kommentare aus einem anderen Blickwinkel.

Ebenso bedanke ich mich bei meinen Kolleginnen und Kollegen am Graduiertenkolleg für die gemeinsame Zeit in Göttingen. Danke euch allen. Besonders möchte ich mich bei Natalia Schlichter für die Unterstützung und die anregenden Gespräche bedanken. Mit ihrer herzlichen und aufbauenden Art hat sie mir allzu oft auch aus schwierigen Phasen geholfen. Ich nehme viele schöne Erinnerungen mit. Danke!

Ein großer Dank geht auch an Dr. Andrew Elliot für die großartigen Monate an der University of Rochester. Dadurch hatte ich die Möglichkeit, einen Einblick in Andrews beeindruckende Arbeit zu bekommen und mich mit ihm und seinem gesamten Team auszutauschen. Vielen Dank für die Gastfreudschaft und für die vielen Eindrücke. Es war eine unvergesslich schöne Zeit.

Ganz besonders danke ich meinen Eltern Marion und Karl-Ernst Paulick für die Unterstützung und Hilfe in allen Bereichen meines Lebens. Schließlich möchte ich mich bei all meinen Freunden für die Unterstützung und Abwechslung bedanken. 


\section{Inhalt}

Verzeichnis der Tabellen ......................................................................................................VIII

Verzeichnis der Abbildungen .......................................................................................................IX

Verzeichnis des Anhangs .................................................................................................................. X

1 Einleitung............................................................................................................................... 1

2 Theoretischer Hintergrund ........................................................................................ 7

$2.1 \quad$ Der Grundschulübergang ....................................................................................... 7

2.1.1 Das Schulsystem der Bundesrepublik Deutschland ......................................... 7

2.1.2 Der Übergang aus Schülersicht ................................................................... 9

2.1.3 Überblick über empirische Studien zum Grundschulübergang........................ 11

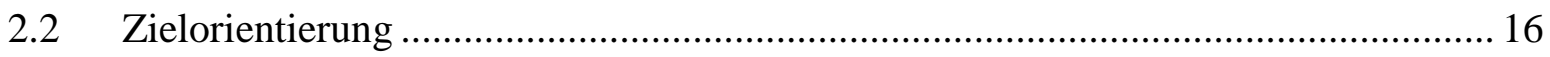

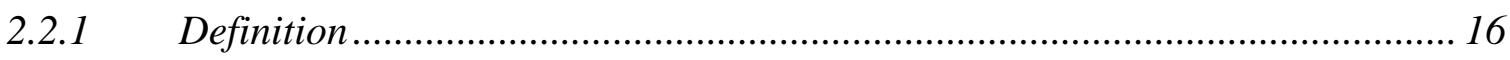

2.2.2 Zielorientierungen und Selbstregulation ........................................................ 18

2.2.3 Zusammenhang mit verschiedenen Variablen ................................................ 19

2.2.3.1 Motivationale, kognitive und behaviorale Variablen ........................................................ 19

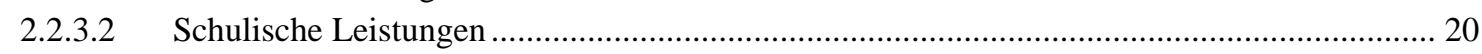

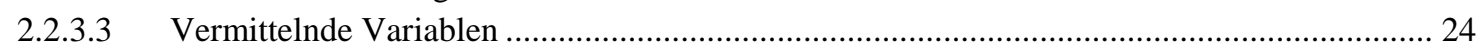

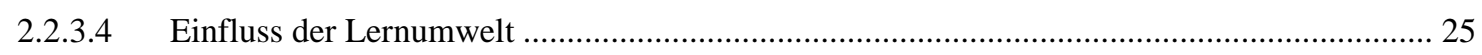

2.2.4 Entwicklung von Zielorientierungen ……………………………………..... 26

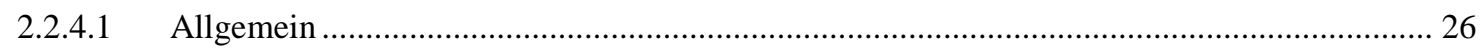

2.2.4.2 Am Übergang zur Sekundarstufe I .................................................................................. 28

2.2.5 Forschungsdesiderat hinsichtlich der Zielorientierungen am

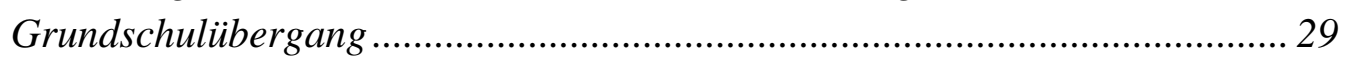

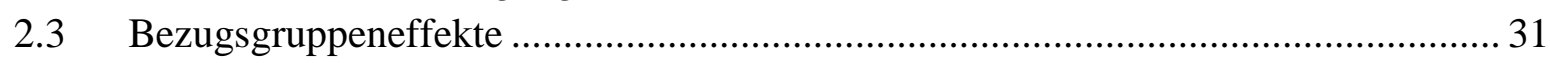

2.3.1 Der Big-Fish-Little-Pond-Effekt.................................................................. 31

2.3.2 Der Basking-In-Reflected-Glory-Effekt ....................................................... 33

2.3.3 Forschungsdesiderat hinsichtlich Bezugsgruppeneffekten bei

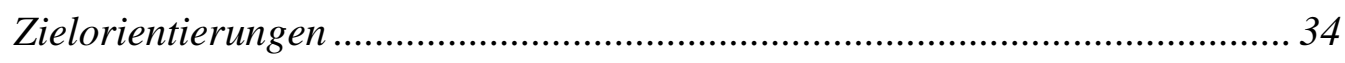

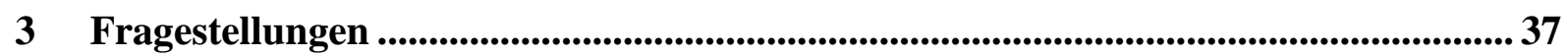

3.1 Ableitung der Fragestellungen.......................................................................... 37

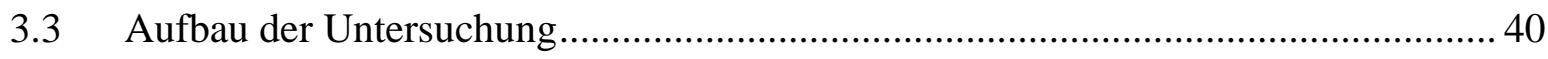

4 Zielorientierungen und schulische Leistungen am Grundschulübergang (Studien

1 und 2).................................................................................................................................4 47

4.1 Zusammenfassung und Abstract...................................................................... 47

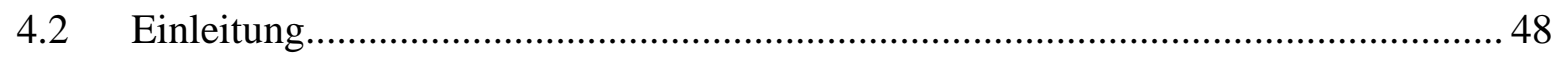

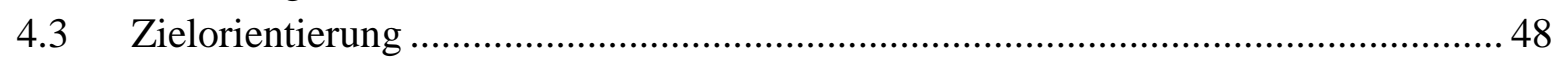

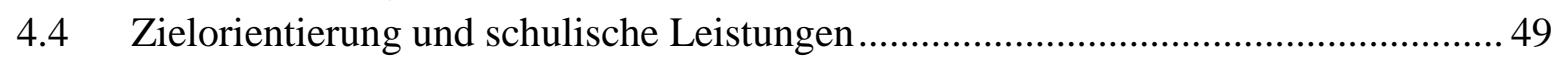

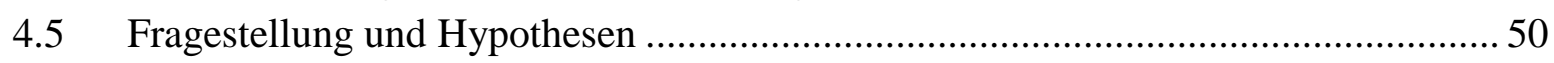

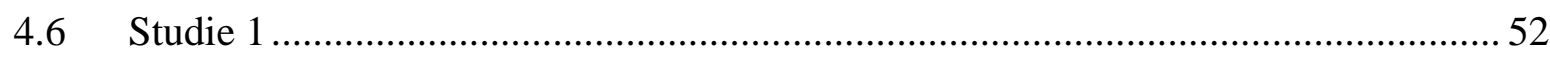

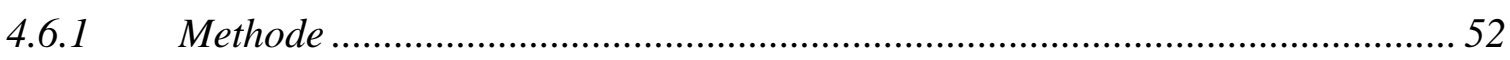




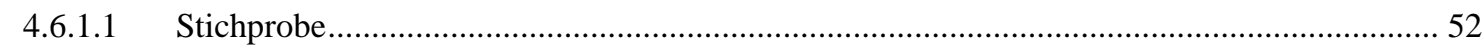

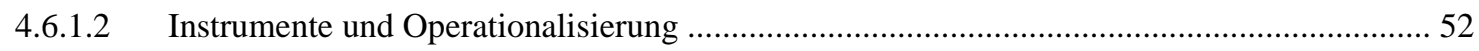

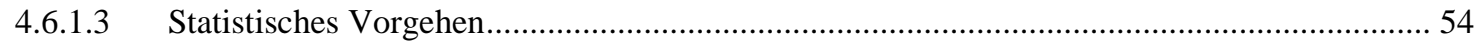

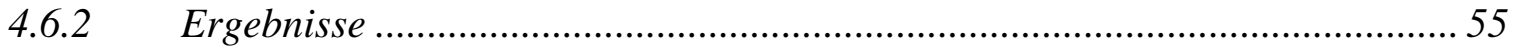

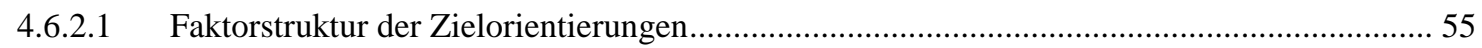

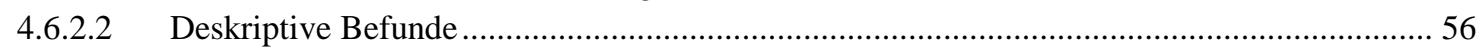

4.6.2.3 Zusammenhang zwischen Zielorientierungen und Schulleistungen................................... 56

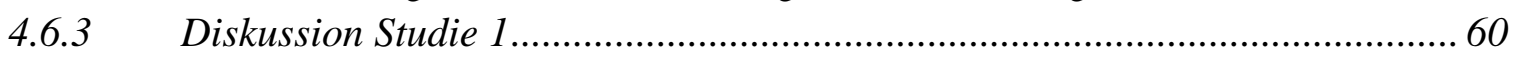

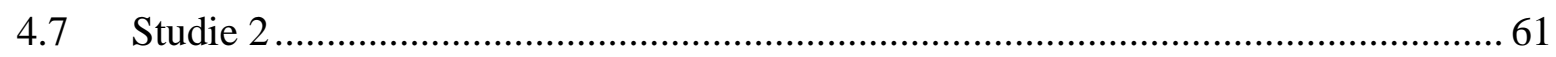

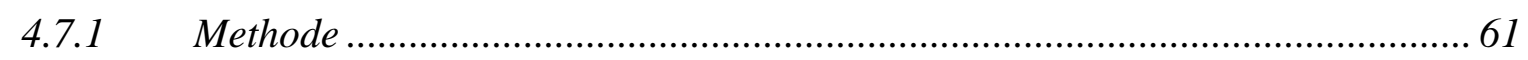

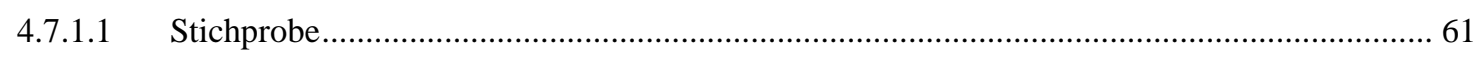

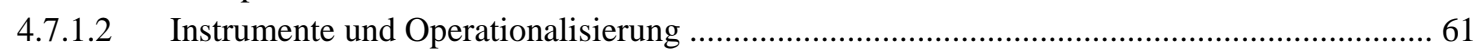

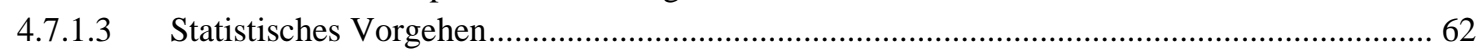

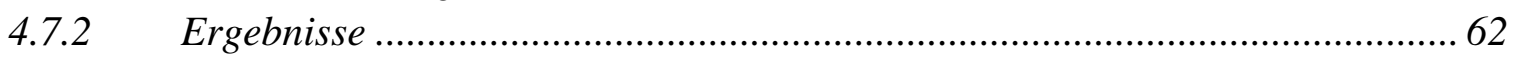

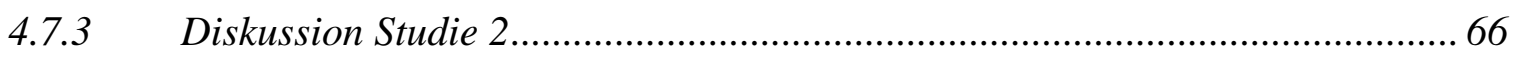

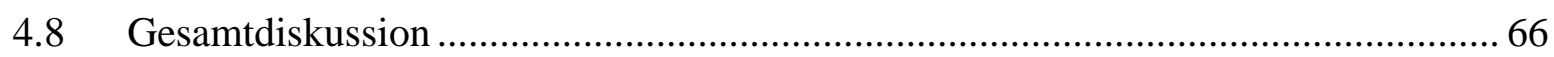

5 Achievement goals and school achievement: The transition to different school

tracks in secondary school (Studie 3) ................................................................................. 71

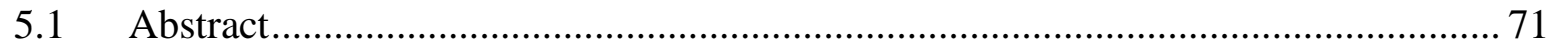

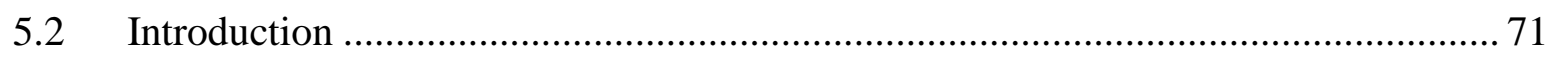

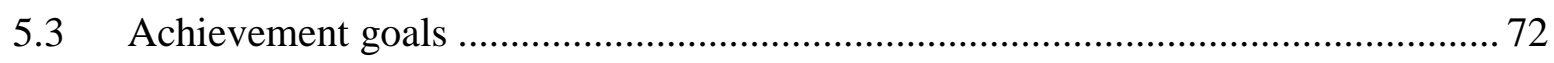

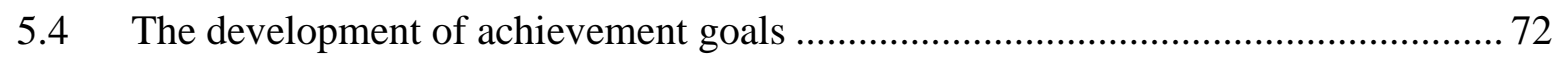

5.5 The relation between achievement goals and school achievement ........................... 75

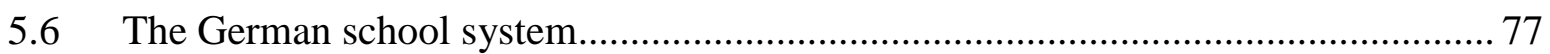

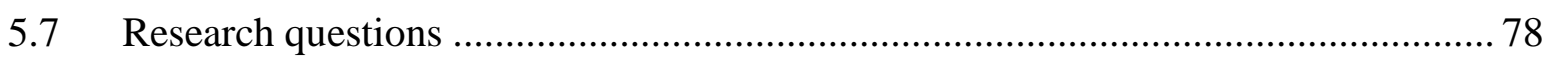

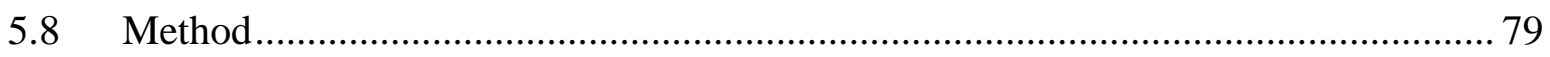

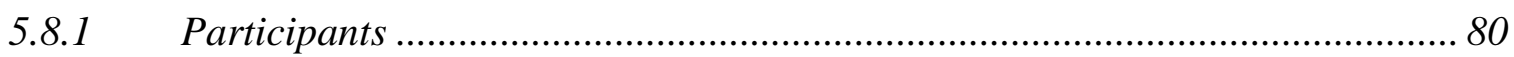

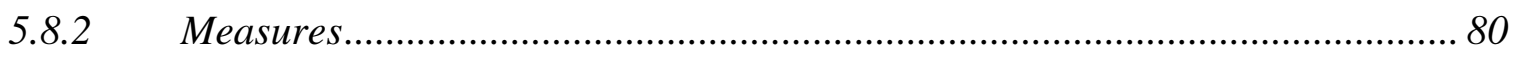

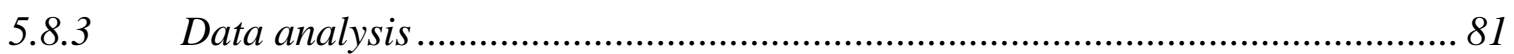

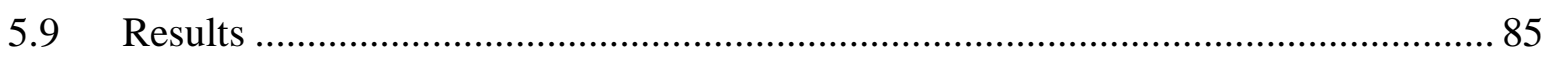

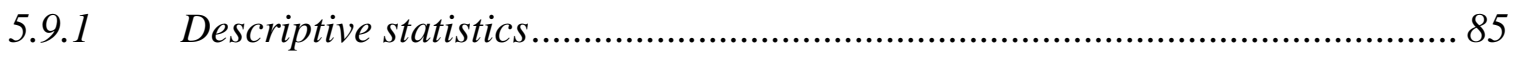

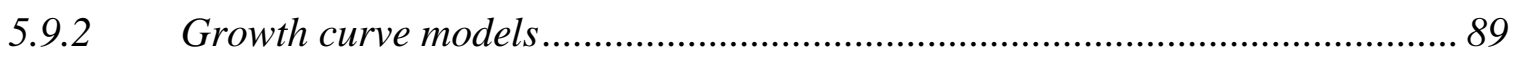

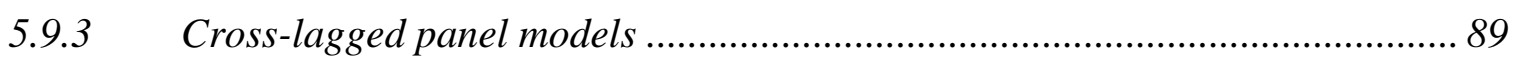

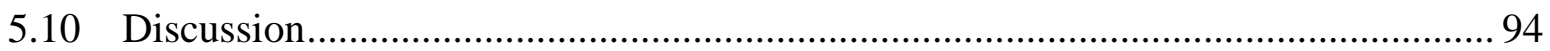

6 Effects of tracking on students's self-concept, intrinsic motivation, and achievement goals during the transition to secondary school (Studie 4) .................... 99

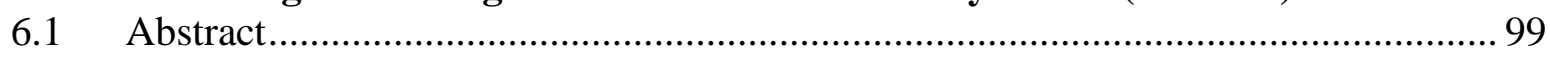

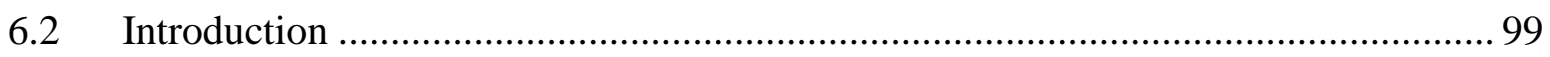

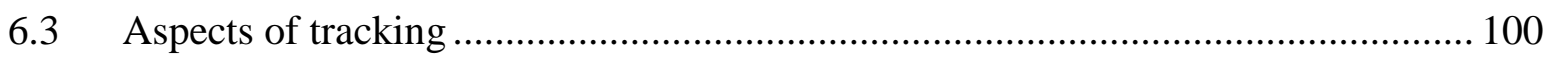

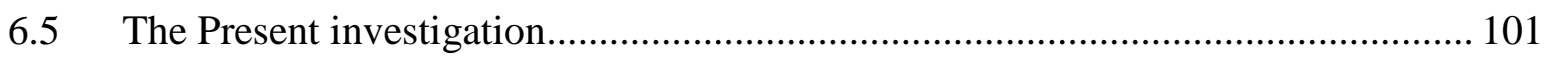

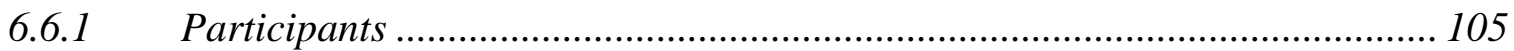

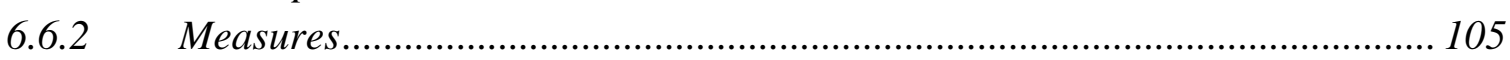

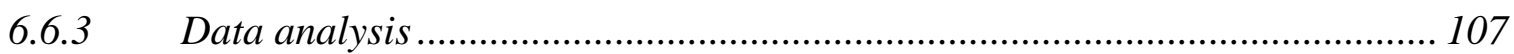

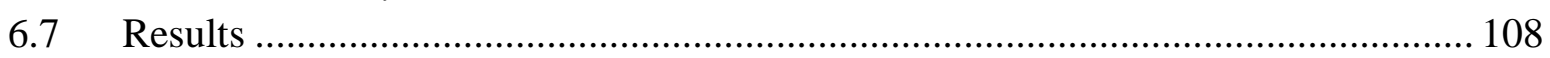




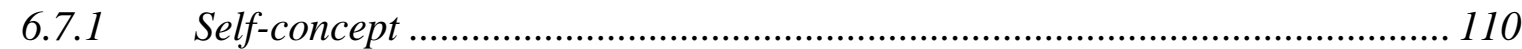

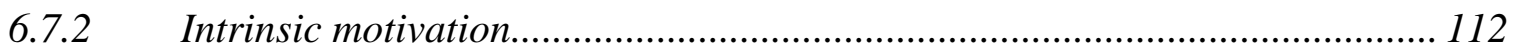

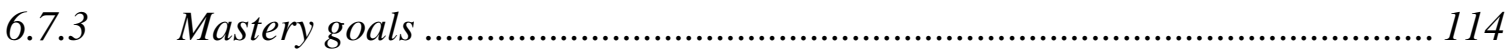

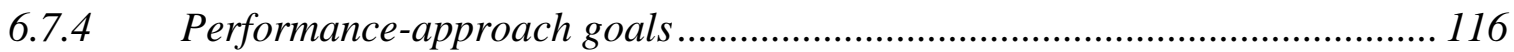

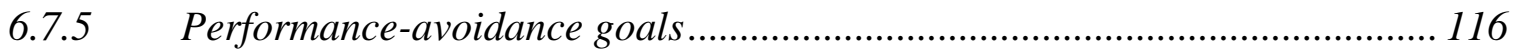

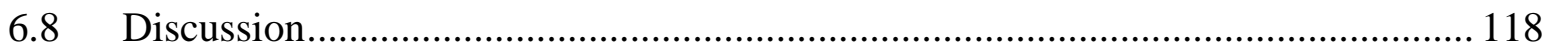

7 Zusammenfassende Schlussdiskussion und Ausblick ................................................. 123

7.1 Zusammenfassung der zentralen Ergebnisse ...................................................... 123

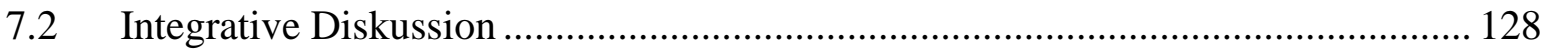

7.3 Grenzen der Arbeit und Ansätze für die weitere Forschung ................................ 140

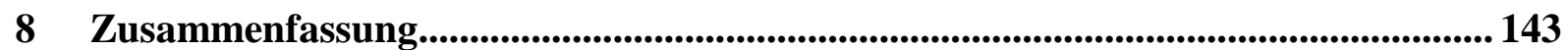

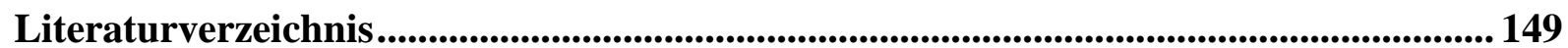

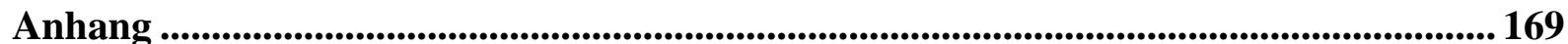




\section{Verzeichnis der Tabellen}

Tabelle 1 Deskriptive Statistiken und Interkorrelationen (Produkt-Moment-

Korrelationen)

Tabelle 2 Zusammenfassung der multiplen linearen Regression zur Vorhersage der schulischen Leistungen (standardisierte Koeffizienten).....

Tabelle 3 Deskriptive Statistiken und Interkorrelationen (Produkt-Moment-

Korrelationen)

Tabelle 4 Zusammenfassung der multiplen linearen Regression zur Vorhersage der schulischen Leistungen (standardisierte Koeffizienten).....

Tabelle 5 Descriptive statistics and reliabilities among achievement goals and GPAs at academic and non-academic tracks from $\mathrm{T} 1$ to $\mathrm{T} 3$

Tabelle 6 Pearson-correlation coefficients among measures.

Tabelle 7 Test of model fit and chi-square difference test for the cross-lagged panel

models

Tabelle 8 Unstandardized and standardized path coefficients for the final two-group

cross-lagged panel model

Tabelle 9 Descriptive statistics for self-concept, intrinsic motivation, achievement goal variables, test scores, and GPAs

Tabelle 10 Results of the conditional latent growth curve models for

students`self-concept

Tabelle 11 Results of the conditional latent growth curve models for students' intrinsic motivation

Tabelle 12 Results of the conditional latent growth curve models for students' mastery goals.

Tabelle 13 Results of the conditional latent growth curve models for students'

performance-approach

Tabelle 14 Results of the conditional latent growth curve models for students' performance-avoidance goals 


\section{Verzeichnis der Abbildungen}

Abbildung 1 A priori model of causal paths between GPAs (GPA) and achievement goals (goal). For clarity, only a general achievement goal factor was pictured (goal) rather than all three types of achievement goals (mastery, performance-approach and performance-avoidance goals). Achievement goals were inferred from four indicators for mastery/performance-approach goals and two indicators from performance-avoidance goals.

Abbildung 2 Means of achievement goals and GPA from grades four to six for academic and non-academic tracks. T1 $=$ End of grade four. T2 $=$ Middle of grade five. $\mathrm{T} 3=$ Beginning of grade six. 


\section{Verzeichnis des Anhangs}

Anhang A: Itemübersicht und Ergebnisse der konfimatorischen Faktorenanalyse

(standardisierte Faktorladungen) 169

Anhang B: Itemübersicht Studie 1: Intrinsische Motivation, akademisches Selbstkonzept und Leistungsangst 170

Anhang C: Übersicht der eingesetzten SELLMO-Items 171 


\section{$1 \quad$ Einleitung}

Zielorientierungen gelten als ein bedeutsames Konzept für schulisches Lernen (vgl. z.B. Schunk, Pintrich \& Meece, 2008). Dabei wird unter dem Konzept der Zielorientierung die motivationale Ausrichtung von Personen in Lern- und Leistungskontexten verstanden, welche eine wichtige Determinante des Leistungshandelns darstellt (z.B. Harackiewicz, Barron, Tauer \& Elliot, 2002). Diesbezüglich werden verschiedene Formen von Zielorientierungen unterschieden. So kann zum einen der Fokus auf Kompetenzsteigerung liegen (Lernzie$\left.1 \mathrm{e}^{1}\right)$, zum anderen auf das Demonstrieren eigener Kompetenzen bzw. das Verbergen fehlender Kompetenzen (Annäherungs- bzw. Vermeidungsleistungsziele). Die unterschiedliche motivationale Ausrichtung zieht dabei Unterschiede im Erleben und Lernverhalten der Personen nach sich, was sich wiederum auf den Lernerfolg und die Leistungsentwicklung auswirken kann (Ames, 1992; Dweck \& Leggett, 1988; Nicholls, 1984). So erwiesen sich Lernziele häufig als adaptiv bzw. förderlich in Bezug auf schulisches Lernen, wohingegen das Zusammenhangsmuster für Leistungsziele (insbesondere für Vermeidungsleistungsziele) eher in die entgegengesetzte Richtung deutete (z.B. Elliot \& Church, 1997; Harackiewicz, Barron, Carter, Lehto \& Elliot, 1997; Lemos, 1999).

Trotz umfangreicher Forschung zum Thema Zielorientierung besteht in vielen Bereichen Forschungsbedarf, insbesondere was die Untersuchung von Zielorientierungen bei jungen Schülern ${ }^{2}$ der Primarstufe bzw. zu Beginn der Sekundarstufe I betrifft. Ein großer Teil der Zielorientierungsforschung bezog sich bislang auf ältere Schüler (weiterführende Schule bzw. Studenten) (siehe Metaanalyse von Hulleman, Schrager, Bodmann \& Harackiewicz, 2010). Die Erforschung von Zielorientierungen im Primarbereich wurde hingegen oftmals vernachlässigt. Zwar finden sich insbesondere im anglo-amerikanischen Raum zahlreiche Studien zum Grundschulübergang (z.B. Anderman \& Midgley, 2004; Wigfield, Eccles, McIver, Reuman \& Midgley, 1991), allerdings wurde wenig Gewicht auf Zielorientierungen gelegt. Vielmehr standen beispielsweise das Fähigkeitsselbstkonzept, die intrinsische Motivation

\footnotetext{
${ }^{1}$ Für die Begriffe Lernzielorientierung, Annäherungsleistungszielorientierung und Vermeidungsleistungszielorientierung werden in der vorliegenden Dissertation die kürzeren Formen Lernziele, Annäherungsleistungsziele und Vermeidungsleistungsziele synonym verwendet.

${ }^{2}$ In dieser Dissertation wird aus Gründen der besseren Lesbarkeit nur die männliche Geschlechtsform gewählt; es sind jedoch stets beide Geschlechter gemeint.
} 
oder die Schulleistungen im Zentrum der Untersuchungen. Aber auch im deutsch-sprachigen Raum ist der Forschungsstand zu Zielorientierungen am Ende der Grundschulzeit bzw. zu Beginn der Sekundarstufe I lückenhaft. So bezogen sich die im deutschen Sprachraum durchgeführten Untersuchungen an dieser Altersgruppe entweder auf sehr kleine, homogene Stichproben (Finsterwald, 2006, Schöne, 2007) oder lediglich auf einzelne Schulfächer (Schwinger \& Wild, 2006). Des Weiteren wurde der Grundschulübergang in dem Zusammenhang nur unzureichend in den Blick genommen.

Der Übergang von der Grundschule auf die weiterführende Schule stellt jedoch ein bedeutsames und wichtiges Ereignis im Leben eines jeden Schülern dar, welches mit zahlreichen Veränderungen einhergeht. Die Bewältigung des Grundschulübergangs gestaltet sich allerdings nicht für jeden Schüler in befriedigender Weise, weshalb diesbezüglich in der Literatur oft von einem „kritischen Lebensereignis“ (Filipp, 1995) gesprochen wird. Im internationalen Vergleich stellt der Grundschulübergang in der Bundesrepublik Deutschland eine Besonderheit in mindestens zwei Punkten dar. Anders als in vielen anderen Ländern wechseln die bundesdeutschen Schüler zum einen bereits nach Klassenstufe vier ${ }^{3}$ auf die weiterführende Schule. Zum anderen gliedert sich das deutsche Sekundarstufensystem in verschiedene Schulformen. Die Dreigliedigkeit mit der Einteilung in Gymnasium, Realschule und Hauptschule ist das wohl bedeutendste Merkmal des deutschen Schulsystems mit für die Schüler weitreichenden Konsequenzen für ihre weitere Laufbahn. So unterscheiden sich die Bildungswege an den jeweiligen Schulformen deutlich in ihrem Anspruchsniveau und in den für die spätere berufliche Karriere relevanten Perspektiven. Dabei basiert die Übergangsempfehlung größtenteils auf den in der Grundschule erbrachten Leistungen der Schüler, was eine damit einhergehende Leistungshomogenisierung und eine Selektion der Schüler in der Bundesrepublik zu einem sehr frühen Zeitpunkt bedeutet.

In zahlreichen Untersuchungen wurde mit dem Grundschulübergang ein Absinken der Lernmotivation verbunden (z.B. Anderman \& Maehr, 1994; Eccles \& Midgley, 1989; Watermann, Klingebiel \& Kurtz, 2010). Durch die sich im Laufe der Entwicklung verändernden Schülerbedürfnisse in Richtung eines zunehmenden Wunsches nach Selbstbestimmung und den mit dem Grundschulübergang einhergehenden Veränderungen der Lernumwelten - oftmals in eine tendenziell entgegengesetzte Richtung - kann es zu einer fehlenden Passung zwischen Schülerbedürfnissen und der Lernumgebung kommen (stage-environment-fit theory,

\footnotetext{
${ }^{3}$ Eine Ausnahme bilden die Bundesländer Berlin und Brandenburg, in denen die Grundschulzeit sechs Jahre beträgt.
} 
Eccles et al., 1993). Dies kann eine Abnahme motivationsbezogener Schülermerkmale verursachen. In diesem Zusammenhang stellt die Erforschung der Entwicklung von Zielorientierungen - welche ein motivationsbezogenes Schülermerkmal darstellen - am Übergang von der Grundschule auf die verschiedenen Schulformen der Sekundarstufe I ein Forschungsdesiderat dar, welches mit der vorliegenden Arbeit behoben werden soll.

Ziel dieser Dissertation soll es sein, zum einen die Bedeutung von Zielorientierungen am Ende der Grundschulzeit zu untersuchen und zum anderen die Entwicklung dieser am Übergang zu den verschiedenen Schulformen zu analysieren. Weiterhin soll die Frage nach dem Zusammenhang zwischen Zielorientierungen und schulischen Leistungen sowohl vor als auch nach dem Grundschulübergang beantwortet werden. Auch diesbezüglich soll untersucht werden, ob schulformspezifische Unterschiede bestehen.

Da sich der Grundschulübergang als ein bedeutsames Lebensereignis mit unter Umständen weitreichenden Auswirkungen auf motivationale, emotionale und kognitive Merkmale der Schüler erwiesen hat, soll in Kapitel 2.1 zunächst genauer auf das bundesdeutsche Schulsystem eingegangen werden. Dabei werden die für das deutsche Schulsystem charakteristischen Merkmale hervorgehoben, der Grundschulübergang aus Schülersicht beleuchtet und ein kurzer Überblick über empirische Studien zu diesem Thema gegeben. Im Kapitel 2.2 soll dann allgemein das Konstrukt der Zielorientierungen und der Zusammenhang zwischen Zielorientierungen und verschiedenen Variablen des schulischen Lernens dargestellt werden. Weiterhin wird der Forschungsstand zu Zielorientierungen im Primarbereich bzw. zur Entwicklung von Zielorientierungen allgemein und am Grundschulübergang erläutert.

Die Untersuchung von Bezugsgruppeneffekten am Grundschulübergang ist ein weit erforschtes Feld. Diesbezüglich wurden unter anderem Fähigkeitsselbstkonzept, intrinsische Motivation, Interesse oder Bildungsaspirationen in den Blick genommen (z.B. Marsh, Köller \& Baumert, 2001; Trautwein, Lüdtke, Marsh, Köller \& Baumert, 2006), jedoch kaum Merkmale darüber hinaus. So besteht Forschungsbedarf darin, mögliche Effekte eines Bezugsgruppenwechsels auf andere Variablen des schulischen Lernens wie beispielsweise Zielorientierungen auszuweiten. Da Zielorientierungen zum einen als motivationale Ausrichtungen von Personen gelten und sie zum anderen oftmals ein Zusammenhang mit Selbstkonzept, intrinsische Motivation oder Interesse aufweisen, liegt die Vermutung nahe, dass sich ein Bezugsgruppenwechsel, wie er mit dem Grundschulübergang verbunden ist, ähnlich wie auf Selbstkonzept oder Interesse möglicherweise auch auf Zielorientierungen an der jeweiligen Schulform auswirkt. Welche Formen von Bezugsgruppeneffekten Einfluss auf die Entwicklung von Zielorientierungen nehmen könnten, wird in Kapitel 2.3 näher erläutert. Zum einen wird hier- 
bei auf den Big-Fish-Little-Pond-Effekt (BFLPE; Marsh, 1987) und zum anderen auf den Basking-in-Reflected-Glory-Effekt (Cialdini et al., 1976) eingegangen. Ausgehend von den theoretischen Aspekten und den empirischen Befunden werden in Kapitel 3 die für die vorliegende Arbeit zentralen Fragestellungen abgeleitet und spezifiziert. Anschließend soll die Beantwortung der jeweiligen Forschungsfragen Inhalt der folgenden Kapitel sein. So steht die Reliabilität und Konstruktvalidität von Zielorientierungen und deren Zusammenhang mit schulischen Leistungen am Ende der Grundschulzeit im Zentrum der ersten Studie von Kapitel 4, bevor in Studie 2 die Replikation der Befunde angestrebt wird. Lassen sich Zielorientierungen bereits am Ende der Grundschulzeit bzw. zu Beginn der Sekundarstufe I reliabel und valide messen und in welchem Zusammenhang stehen sie mit schulischen Leistungen? In Kapitel 5 (Studie 3) soll der Frage nachgegangen werden, wie sich Zielorientierungen am Grundschulübergang entwickeln und ob sich schulformspezifische Unterschiede im Entwicklungsmuster ergeben. Zudem ist die Analyse eines sich potenziell verändernden Zusammenhangmusters zwischen Zielorientierungen und schulischen Leistungen Teil dieses Kapitels. Lassen sich auch hier schulformspezifische Unterschiede nachweisen? In Kapitel 6 soll untersucht werden, ob sich auch bei Zielorientierungen ein Bezugsgruppeneffekt zeigt (Studie 4). Dazu werden zunächst die Auswirkungen des Grundschulübergangs auf das Fähigkeitsselbstkonzept und die intrinsische Motivation der Schüler analysiert, bevor die Zielorientierungen in den Blick genommen werden. Eine weitere Forschungsfrage, welcher in Kapitel 6 nachgegangen werden soll, ist die Frage, ob sich der Bezugsgruppeneffekt durch die Kontrolle der Schulnoten in Klassenstufe fünf erklären lässt. Da einige Studien nachweisen konnten, dass der BFLPE über die Schulnoten vermittelt wird (z.B. Trautwein, Lüdtke, Marsh et al., 2006), soll auch in der vorliegenden Arbeit ein möglicher mediierender Effekt der Schulnoten untersucht werden.

Diese vier Studien bilden den Kern der vorliegenden Arbeit und wurden so verfasst, dass sie in Fachzeitschriften zur Veröffentlichung eingereicht werden können. Der Beitrag, der aus den - in Kapitel 4 - vorgestellten Studien 1 und 2 hervorgegangen ist, wurde in der Zeitschrift Unterrichtswissenschaft zur Begutachtung eingereicht. Studien 3 und 4 (Kapitel 5 und 6) wurden in englischer Sprache verfasst, sodass diese beiden Beiträge in internationalen Fachzeitschriften zur Begutachtung eingereicht werden können bzw. wurde die in Kapitel 5 vorgestellte Studie 3 bereits bei der Zeitschrift Contemporary Educational Psychology eingereicht. Die zentralen Ergebnisse der vier Studien werden in Kapitel 7 zusammengefasst und integrativ diskutiert. Abschließend werden die Grenzen der Arbeit aufgezeigt und ein Ausblick für zukünftige Forschung gegeben. 


\section{Theoretischer Hintergrund}

\subsection{Der Grundschulübergang}

Übergänge stellen wichtige und oftmals unausweichliche Lebensereignisse eines jeden Menschen dar. Angefangen im sehr frühen Kindesalter mit dem Übergang in eine Kindertagesstätte bis hin ins hohe Erwachsenenalter mit dem Ausscheiden aus dem Berufsleben in den Ruhestand, ist das Leben von zahlreichen Übergängen geprägt. Nicht selten sind diese Übergänge gesetzlich normiert, institutionalisiert und mitunter ritualisiert, weswegen auch von normativ kritischen Lebensereignissen gesprochen wird (Beelmann, 2000; Tippelt, 2004).

Übergänge sind durch substantielle Veränderungen der Umgebung gekennzeichnet und erfordern eine Anpassung an die veränderte Umgebung. Diese Anpassung wird von der Interaktion zwischen dem Individuum und der neuen Umwelt beeinflusst. Wichtige Faktoren stellen dabei zum einen der Grad der Veränderung durch den Übergang dar und zum anderen persönliche Eigenschaften des Individuums (z.B. Persönlichkeit, Ressourcen, Fähigkeiten und Vorstellungen) (Kelly, Ryan, Altman \& Stelzner, 1993). Diese Faktoren tragen dazu bei, dass eine Anpassung an die veränderte Umgebung gelingt oder scheitert.

Die schulischen Übergänge spielen eine wichtige und wegweisende Rolle im Lebenslauf eines jeden. Besonders der Übergang von der Grundschule auf die weiterführende Schule gilt als eine entscheidende Weichenstellung für den späteren beruflichen Werdegang. Dies lässt sich am engen Zusammenhang zwischen Bildungsabschluss und dem beruflichen Statuserwerb (Tippelt, 2000) ablesen.

\subsubsection{Das Schulsystem der Bundesrepublik Deutschland}

Der Übergang von der Grundschule auf die weiterführende Schule findet in Deutschland für alle Schüler nach Vollendung der vierten Klassenstufe statt ${ }^{4}$ und ist gesetzlich vorgeschrieben. Das Besondere am deutschen Schulsystem stellen hierbei zum einen der relativ frühe Wechsel und zum anderen die Leistungsselektivität des Übertritts in das weiterführende Schulwesen dar. Nach der einheitlichen Grundschule differenziert sich das Schulsystem dreigliedrig, was eine sehr frühe Sondierung der Schüler auf unterschiedlich anspruchsvolle und

\footnotetext{
${ }^{4}$ Ausnahmen bilden die Bundesländer Berlin und Brandenburg.
} 
perspektivenreiche Bildungswege bedeutet und weit reichende Folgen für die Schülerbiografie hat (Kramer, Helsper, Thiersch \& Ziems, 2009). Dabei sind die Regelungen für den Übergang in die weiterführende Schule für die 16 Bundesländer uneinheitlich. Länderspezifische Schulgesetzte sind für die Aufgaben, Strukturen und Inhalte der Schulen verantwortlich (Leschinsky, 2005). Aus diesem Grund ist auch das Schulsystem nicht einheitlich. Grundsätzlich findet man in vielen Bundesländern ein dreigliedriges System mit den Schulformen Hauptschule, Realschule und Gymnasium. Weit verbreitet sind auch Schulen, welche mehrere Bildungsgänge (Hauptschul-, Realschulzweig) anbieten ${ }^{5}$. Eine weitere Schulform stellt die Gesamtschule dar, welche ursprünglich als Ersatz für das dreigliedrige Schulsystem in den 1960er Jahren gegründet wurde, heute jedoch eher in einem Wettbewerb mit diesem steht. Die Gesamtschule vereint sowohl Haupt- und Realschule als auch Gymnasium und ist durch eine vielfältigere und flexible Unterrichtsorganisation innerhalb der Schule gekennzeichnet, welche Jahrgangsklassen, Unterricht in Fachleistungskursen, Wahlpflichtveranstaltungen und Wahlangebote umfasst (Köller, 2005).

Der Übertritt in die verschiedenen Schulformen ${ }^{6}$ der weiterführenden Schule stellt ein Ereignis mit enormer Tragweite dar und gilt als folgenreichstes Beispiel schulischer Differenzierung. Die frühe Leistungsdifferenzierung geht mit dem Erwerb unterschiedlicher Abschlusszertifikate einher, welche über die nachfolgenden Ausbildungsoptionen den beruflichen Werdegang beeinflussen (Maaz et al., 2008). Anders als ursprünglich beabsichtigt, führt die mit der Leistungsdifferenzierung verbundene Homogenisierung oftmals nicht dazu, dass die Schüler bestmöglich gefördert werden, sondern dass frühzeitig Lebenschancen auf der Grundlage kritikwürdiger Übertrittsverfahren vergeben werden (Baeriswyl, Trautwein, Wandeler \& Lüdtke, 2009). Die Ergebnisse einiger Schulleistungsstudien belegen hingegen, dass sich die Leistungsverteilungen an den verschiedenen Schulformen substanziell überlappen, da die Bewertungsstandards innerhalb und zwischen den Schulformen uneinheitlich sind (Baumer, Trautwein, Artelt, 2003). Als umso gravierender können die Folgen der Übergangs-

\footnotetext{
${ }^{5}$ Die Bezeichnungen der Schulen mit mehreren Bildungsgängen unterscheiden sich zwischen den Bundesländern: Mittelschule (Sachsen), Regelschule (Thüringen), Erweiterte Realschule (Saarland), Sekundarschule (Bremen, Sachsen-Anhalt), Integrierte Haupt- und Realschule (Hamburg), Verbundene oder Zusammengefasste Haupt- und Realschule (Berlin, Hessen, Mecklenburg-Vorpommern, Niedersachsen), Regionale Schule (Mecklenburg-Vorpommern, Rheinland-Pfalz), Oberschule (Brandenburg), Duale Oberschule (Rheinland-Pfalz), Regionalschule (Schleswig-Holstein), Gemeinschaftsschule (Schleswig-Holstein) (Quelle: Ständige Konferenz der Kultusminister der Länder in der Bundesrepublik Deutschland, 2010).

${ }^{6}$ Im Folgenden wird die Bezeichnung Schulform synonym sowohl für die verschiedenen Schulformen (Hauptschule, Realschule, Gymnasium) als auch für Schulen mit mehreren Bildungsgängen verwendet.
} 
entscheidung eingeschätzt werden. Diese Übergangsentscheidung kann auf Lehrerempfehlungen, dem Elternwillen oder speziellen Bewährungs- und Beratungsformen basieren, wobei auch hier Unterschiede zwischen den Bundesländern bestehen (für detailliertere Informationen siehe Kropf, Gresch \& Maaz, 2010). In 12 von 16 Bundesländern liegt die Entscheidung bei den Eltern, auch wenn der Elternwille durch einige institutionelle Regelungen eingeschränkt sein kann (Jonkmann, Maaz, McElvany \& Baumert, 2010; van Ophuysen, 2006). So geben die Grundschullehrer eine unverbindliche Empfehlung für eine geeignete Schulform, worauf die Eltern ihre Entscheidung stützen. Da allerdings der reine Elternwille bei der Entscheidung für oder gegen eine Schulform umstritten ist, existiert dieser in Reinform nur noch in wenigen Bundesländern (Rheinland-Pfalz, Nordrhein-Westfalen, MecklenburgVorpommern). In vielen Bundesländern (Niedersachsen, Schleswig-Holstein, Hessen, Bremen, Berlin) besteht die Pflicht zu Beratungsgesprächen zwischen Schule und Eltern. In Baden-Württemberg, Bayern, Saarland, Thüringen und Sachsen basiert die Übergangsempfehlung auf dem Notendurchschnitt und einer Aufnahmeprüfung (Kramer et al., 2009; Liegmann \& Lumer, 2004). Ein Großteil der Eltern (ca. drei Viertel) folgt dabei den Empfehlungen der Grundschullehrer (Bellenberg, 2005).

Insgesamt stellt sich das deutsche Schulsystem als sehr komplex und heterogen dar. Die unterschiedlichen länderspezifischen Übergangsregelungen und die zahlreichen zwischen den Bundesländern variierenden Schulformen stoßen nicht selten auf Kritik, da die Wahl einer geeigneten Schulform dadurch erheblich erschwert wird.

\subsubsection{Der Übergang aus Schülersicht}

Beim Übergang von der Grundschule auf die weiterführende Schule befinden sich die Schüler in einem Entwicklungsabschnitt (Alter ca. 10 Jahre), welcher als „relativ einheitliche, undramatische, mehr oder minder kontinuierliche Wachstums- und Bildungsperiode körperlicher, geistiger wie persönlicher Merkmale“ (Weinert \& Stefanek, 1997, S. 439) verstanden wird und somit keine entwicklungsbedingten Einschnitte bietet. Erst der Grundschulübergang stellt einen solchen Einschnitt dar.

Dieser Übergang ist mit zahlreichen z.B. strukturell-organisatorischen und sozialen Veränderungen verbunden. So findet beispielsweise ein Wechsel vom Klassen- zum Fachlehrerprinzip statt, die Leistungsanforderungen an die Schüler steigen, die Klassengröße und die Anzahl der Schulfächer erhöhen sich und die Schüler sehen sich mit neuen didaktischmethodischen und pädagogischen Konzepten konfrontiert. Diese zahlreichen Veränderungen 
erfordern ein hohes Maß an Anpassung. Da die Entwicklung der Schüler hingegen sehr individuell verläuft und eine große Heterogenität innerhalb der Lerngruppen besteht, erfolgt der Übergang für einige Schüler zu einem falschem Zeitpunkt (Weitzel, 2004). Dies kann bei diesen Schülern dazu führen, dass sie Gefühle von Angst und Bedrohung entwickeln. Aus diesem Grund wird in der Literatur im Zusammenhang mit dem Grundschulübergang häufig von einem „kritischen Lebensereignis“ gesprochen (Filipp, 1995; Sirsch, 2000, 2003).

Unter einem kritischen Lebensereignis wird ein Ereignis verstanden, welches durch Veränderungen der (sozialen) Lebenssituation der Person gekennzeichnet ist und entsprechende Anpassungsleistungen erfordert (Filipp, 1995). Folgende drei Aspekte sind nach Filipp zentrale Aspekte kritischer Lebensereignisse: 1. Sie stellen „die raumzeitliche, punktuelle Verdichtung eines Geschehensablaufs innerhalb und außerhalb der Person dar“, sind 2. „Stadien des relativen Ungleichgewichts“ (S. 24) und 3. werden von affektiven Reaktionen begleitet. In Bezug auf den Grundschulübergang lassen sich diese drei Merkmale wie folgt anwenden: Der Übergang lässt sich zeitlich relativ eng eingrenzen, wobei dieser nicht erst mit dem tatsächlichen Schulwechsel stattfindet, sondern bereits eher mit der Übergangsempfehlung bzw. sobald die Schüler ihre Leistungen als wichtig und bedeutsam für den Schulwechsel wahrnehmen. Auch kann der Grundschulübergang als eine Zeit des relativen Ungleichgewichts betrachtet werden, wenn man die zahlreichen Veränderungen, welche mit dem Übergang verbunden sind, bedenkt. Dieses Ungleichgewicht besteht laut Filipp im Passungsgefüge zwischen Person und Umwelt. Wenn die interne Kongruenz in einem Person-Umwelt-Gefüge, welche ein ,adaptives Funktionieren“ (S. 24) im jeweiligen Umweltkontext ermöglicht, ein Mindestmaß unterschreitet, bedarf es einer Neuorganisation dieses Person-Umwelt-Gefüges. Die Ursache für das Ungleichgewicht kann dabei sowohl in der Person als auch in der Umwelt liegen. Da die Neuorganisation des Person-Umwelt-Gefüges qualitativ-struktureller Veränderungen in der Beziehung zwischen Person und Umwelt bedarf und die vorhandenen Verhaltenssysteme der Person gegebenenfalls nicht immer ausreichen, wird von einem kritischen Ereignis gesprochen. Durch die zahlreichen Veränderungen, die mit dem Grundschulübergang einhergehen, kann insbesondere nach dem Schulwechsel davon ausgegangen werden, dass ein Ungleichgewicht zwischen dem Schüler und seiner Umwelt entsteht. Die sich in der Grundschulzeit bewährten Verhaltensweisen und Handlungsroutinen müssen nun hinterfragt bzw. an die neue Schulsituation angepasst werden. Dies kann zum einen durch eine flexible Zielanpassung (akkomodative Prozesse) vonstattengehen, wonach die Schüler die eigenen Handlungsweisen bzw. Erwartungen in der Lernsituation verändern (Brandstädter, 2007), zum anderen aber auch durch eine hartnäckige Zielverfolgung (Assimilation), bei welcher 
eine Veränderung oder Beeinflussung der neuen Lernumgebung angestrebt wird. Eine weitere Möglichkeit der Anpassung ist aber auch die bewusste Wahl einer vermeintlich zu den individuellen Bedürfnissen passende Schule.

Auch Filipps dritter Aspekt eines kritischen Lebensereignisses, die Tatsache der emotionalen Nichtgleichgültigkeit, lässt sich auf den Grundschulübergang anwenden, denn auch dieser ist oftmals mit affektiven Reaktionen verbunden. Dabei müssen die affektiven Reaktionen nicht zwangsläufig negativ sein, wie der Begriff kritisch vermuten lässt, sondern können durchaus von positiver Qualität sein. Demnach lässt sich der Grundschulübergang unter entwicklungspsychologischer Perspektive als eine normativ kritische Entwicklungsaufgabe mit potentiell positiven und negativen Auswirkungen auf das Individuum definieren.

\subsection{3 Überblick über empirische Studien zum Grundschulübergang}

Anschließend soll ein Überblick über empirische Studien zum Grundschulübergang gegeben werden. Dabei sind Studien aus dem anglo-amerikanischen Raum besonders zahlreich. Die groß angelegte Michigan Study of Adolescent Life Transition (MSALT) stellt in diesem Zusammenhang die wohl einflussreichste Studie zum Grundschulübergang dar. Über 3000 Schüler wurden über einen Zeitraum von vier Jahren (ab Klassenstufe sechs) beobachtet. Ziel der Studie der Forschergruppe um Eccles, Midgley und Wigfield war es, den Einfluss von Veränderungen in schulischer als auch familiärer Umwelt auf die Entwicklung unterschiedlicher leistungsbezogener motivationaler, behavioraler und emotionaler Variablen der Schüler zu untersuchen. In zahlreichen Publikationen (z.B. Midgley, Feldlaufer \& Eccles, 1989; Wigfield et al., 1991) wurde der Einfluss des Übergangs von der Grundschule (elementary school) zur weiterführenden Schule (junior high school) dargestellt. Das zentrale Ergebnis der Untersuchungen war, dass nach dem Grundschulübergang bei vielen Schülern eine Minderung u.a. der intrinsischen Motivation, des Fähigkeitsselbstkonzepts, des Interesses und des Selbstvertrauens eingesetzt hat. Als Erklärung wurden zum einen entwicklungsbedingte Veränderungen, zum anderen kontextuelle, die Sekundarstufe betreffende Veränderungen der Lernumwelt verwendet. Zudem wird der Zeitpunkt des Übergangs (nach Klassenstufe sechs) in einem Alter der Schüler von zwölf Jahren von der Forschergruppe als ungünstig eingestuft, da mit einsetzender Pubertät das Bedürfnis nach Selbstbestimmung steigt (selfdetermination theory, Deci \& Ryan, 1985). Dieses Bedürfnis kann jedoch von der Sekundarschule oft nur unzureichend befriedigt werden, da sich die Eigenschaften der weiterführenden Schule vor allem durch ein höheres Maß an Strukturierung, Kontrolle, Regeln und Disziplin 
auszeichnen, was dem steigenden Bedürfnis nach Autonomie entgegensteht. Zudem wird das Schulklima formeller und unpersönlicher, was im Widerspruch zu dem Bedürfnis der Schüler nach sozialer Eingebundenheit und persönlicher Zuwendung steht. Hinzukommt, dass durch gesteigerte Leistungsanforderungen an der weiterführenden Schule dem Bedürfnis nach Kompetenzerleben auf Schülerseite oftmals nicht gerecht wird. Die fehlende Passung zwischen den sich verändernden Schülerbedürfnissen auf der einen Seite und der Lernumwelt an der Sekundarschule auf der anderen Seite wurde in zahlreichen Studien, mit dem Ziel einer Optimierung dieser Passung, untersucht (stage-environment-fit; Eccles et al., 1993). Soll es zu einer pädagogisch wünschenswerten Entwicklung beispielsweise der intrinsischen Motivation oder des Fähigkeitsselbstkonzepts der Schüler kommen, ist es von großer Bedeutung, dass die Lernumwelt den Bedürfnissen der Schüler gerecht wird.

Das US-amerikanische Schulsystem unterscheidet sich in vielen Punkten von dem bundesdeutschen Schulsystem, daher können die Befunde der MSALT-Studie nicht ohne weiteres auf die hiesigen Schüler übertragen werden. Zum einen findet der Wechsel von der Grundschule auf die weiterführende Schule in Deutschland in den meisten Bundesländern bereits nach der vierten und nicht wie in vielen US-Bundesstaaten nach der sechsten Klassenstufe statt. Zum anderen ist das Schulsystem der Bundesrepublik durch seine Dreigliedrigkeit und die damit einhergehende Leistungsselektivität charakterisiert, im US-amerikanischen Raum findet hingegen keine leistungsbasierte Selektion in verschiedene Schulformen statt. Ungeachtet der Leistungen der Schüler findet je nach Schulbezirk ein Wechsel zur junior high school bzw. zur middle school statt. Dort werden die Schüler in für ihr Alter entsprechenden Schulstufen gemeinsam unterrichtet. In den USA sind im Vergleich zur Bundesrepublik Deutschland „within-class, couse-level, and implicit school-level tracking“ (Maaz, Trautwein, Lüdtke \& Baumert, 2008, S. 100) üblicher. Somit lassen sich das US-amerikanische und das bundesdeutsche Schulsystem insbesondere in Hinblick auf den Grundschulübergang nur eingeschränkt gleichsetzen.

Im deutschsprachigen Raum wurde der Übergang zur weiterführenden Schule weniger untersucht, wobei in jüngerer Zeit die Studien zu diesem Thema zunehmen, nicht zuletzt auch aus Kritik am dreigliedrigen Schulsystem. Die im Folgenden vorgestellten Untersuchungen beziehen sich auf den Grundschulübergang aus emotional-motivationaler und leistungsbezogener Sicht. Es wird sich lediglich auf Studien bezogen, welche mindestens einen der für die Fragestellung der vorliegenden Arbeit relevanten motivationalen, emotionalen oder kognitiven Merkmale betrachten. 
Buff (1991) untersuchte das Fähigkeitsselbstkonzept von Schweizer Schülern am Übergang von der Grundschule auf die zweigliedrige Sekundarstufe I im Kanton Bern. Bereits vor dem Grundschulübergang verfügten künftige Schüler der höheren Schulform über ein ausgeprägteres Selbstkonzept als künftige Schüler der niedrigeren Schulform. Nach dem Wechsel auf die weiterführende Schule sank jedoch das Selbstkonzept bei Schülern der höheren Schulform, wohingegen das Selbstkonzept bei Schülern der anderen Schulform anstieg.

In einer österreichischen Studie von Sirsch (2000) wurde die subjektive Bedeutung des Grundschulübergangs betrachtet. Das Schulsystem in Österreich ist in einigen Bereichen gut mit dem bundesdeutschen Schulsystem vergleichbar. Der Grundschulübergang findet ebenfalls nach der vierten Klassenstufe statt und die Schüler wechseln in ein mehrgliedriges Sekundarschulsystem: entweder in die Hauptschule, in die Unterstufe einer allgemeinbildenden höheren Schule oder in die Mittelschule. Das zentrale Ergebnis der Studie war, dass für viele Schüler der Übergang mit einer positiven emotionalen Haltung und einer höheren Herausforderung sowie geringen Bedrohung verbunden wurde. Das Erleben der Schüler war dabei zum einen von der zukünftigen Schulform und zum anderen von individuellen Veranlagungen und Persönlichkeitsmerkmalen abhängig.

Der Einfluss des Grundschulübergangs auf die psychische Anpassung der Schüler wurde von Elben, Lohaus, Ball und Klein-Heßling (2003) untersucht. Dabei wurden am Ende der Grundschulzeit und zu Beginn der Sekundarstufe I somatische und psychische Symptome der Schüler erfasst. Insgesamt kam es zu einer Abnahme der Symptome nach dem Grundschulübergang unabhängig vom besuchten Schultyp. Zudem zeigte sich ein geschlechterspezifischer Unterschied: die Symptomatik sank bei Schülerinnen stärker ab als bei Schülern. Die Ergebnisse deuteten auf einen Erholungseffekt hin, welcher durch die Schulferien begünstigt wurde.

Valtin und Wagner (2004) untersuchten in ihrer Längsschnittstudie SABA (Schulische Adaptation und Bildungsaspiration) die Auswirkungen des Schulwechsels auf die Entwicklung leistungsbezogener Persönlichkeitsmerkmale von Schülern der Klassenstufe vier bis sieben. Die Studie ergab, dass sich Schüler unterschiedlicher Schulformen in ihren Entwicklungsverläufen signifikant voneinander unterschieden. So verfügten angehende Gymnasiasten in der vierten Klassenstufe über ein höheres Fähigkeitsselbstkonzept und Selbstwertgefühl, günstigere Attributionsmuster bei Misserfolgen und weniger Leistungsangst als angehende Hauptschüler. Die Werte der Schüler an den verschiedenen Schulformen glichen sich nach dem Grundschulübergang (in der siebten Klassenstufe) jedoch an, sodass die Persönlichkeitsentwicklung von Hauptschülern insgesamt positiver verlief. 
Im Dortmunder DfG-Projekt „Übergang von der Grundschule zur weiterführenden Schule“ entstanden eine Reihe von Publikationen, welche sich mit dem Grundschulübergang beschäftigt haben. Beispielsweise untersuchten Harazd und Schürer (2006) die Entwicklung von Schulfreude am Grundschulübergang. Insgesamt zeigten die Befunde, dass die Schulfreude von zukünftigen Hauptschülern in Klassenstufe vier am geringsten war, dass diese nach dem Übergang in Klassenstufe fünf allerdings im Vergleich zu Gesamtschülern und Gymnasiasten am größten war. Als bedeutsame Prädiktoren erwiesen sich dabei die Prüfungsangst der Schüler, ihr Verhältnis zur Lehrkraft, das Fähigkeitsselbstkonzept und das Klassenklima. Nach dem Wechsel auf die weiterführende Schule hatten das Fähigkeitsselbstkonzept und das Schulklima allerdings keinen Effekt mehr auf die Schulfreude der Schüler.

Van Ophuysen (2008) beschäftigte sich im Rahmen des Dortmunder DfG-Projekts mit der Entwicklung der affektiven Einstellung von Schülern am Grundschulübergang (Klassenstufe vier bis sieben). Die affektive Einstellung der Schüler verbesserte sich jeweils nach den Ferien, die Ergebnisse deuten somit auf einen Erholungseffekt. Dieser Effekt war nach dem Grundschulübergang noch größer und ging signifikant über die ferienbedingten Erholungseffekte hinaus. Dabei konnte der Effekt an allen Schulformen nachgewiesen werden. Insgesamt war die Schulfreude der zukünftigen Gymnasiasten in der vierten Klassenstufe größer als die der späteren Hauptschüler. Am Ende der Untersuchung in der siebten Klassenstufe gab es allerdings keine bedeutsamen Unterschiede mehr zwischen Schülern der jeweiligen Schulformen.

Finsterwald (2006) untersuchte die motivationale Bewältigung des Grundschulübergangs von $N=184$ Schülern und fand bereits vor dem Übergang ein günstigeres adaptives Leistungshandeln bei zukünftigen Gymnasiasten (hohes Vertrauen in die eigenen Fähigkeiten, hohe schulische Leistungen, hingegen gering ausgeprägte Hilflosigkeit und leistungsbezogenen Ängste) im Vergleich zu künftigen Gesamt- oder Realschülern. In Bezug auf motivationale Bedingungsfaktoren (Zielorientierungen) zeigten künftige Realschüler ein eher ungünstiges Bild, bei ihnen waren Annäherungsleistungsziele am stärksten ausgeprägt. Nach dem Grundschulübergang zeigte sich bei den Gymnasiasten hinsichtlich der verschiedenen Variablen eines adaptiven Leistungshandelns eine Verschlechterung, wohingegen der Übergang für die Gesamtschüler kurz- und langfristig am positivsten verlief.

In einer mikrogenetischen Längsschnittstudie von Aust, Watermann und Grube (2010) wurde die Entwicklung des Fähigkeitsselbstkonzepts kurze Zeit nach dem Grundschulübergang zu zwei verschiedenen Schulformen (Gymnasium, Integrierte Gesamtschule) untersucht. Das zentrale Ergebnis der Studie war, dass bereits kurze Zeit nach dem Übergang das Selbst- 
konzept am Gymnasium stärker als an der Gesamtschule abnahm (Big-Fish-Little-PondEffekt; BFLPE; Marsh, 1987), jedoch näherten sich die Selbstkonzepte in den beiden Gruppen im weiteren Verlauf an. Lernziele und auch (auf dem Gymnasium) Annäherungsleistungsziele hingen zu Beginn der Untersuchung positiv mit dem Selbstkonzept zusammen. Außerdem zeigte sich in der Gesamtschule ein positiver Zusammenhang zwischen Vermeidungsleistungszielen und der Selbstkonzeptentwicklung.

In der groß angelegten Längsschnittstudie Der Übergang von der Grundschule in die weiterführende Schule - Leistungsgerechtigkeit und regionale, soziale und ethnisch-kulturelle Disparitäten (Maaz, Baumert, Gresch \& McElvany, 2010), welche eine Erweiterung der Trend in International Mathematics and Science Study 2007 darstellt und am Berliner MaxPlanck-Institut für Bildungsforschung durchgeführt wurde, war es erstmals möglich, bundesweit repräsentative Daten für den Übergang in die weiterführende Schule zu erfassen. Ziel dieser Studie war es, Übergangsentscheidungen am Ende der Grundschulzeit in Bezug auf verschiedene Zusammenhangsfaktoren (z.B. bisheriges Leistungs- und Arbeitsverhalten der Schüler, elterliche Willensbildung in Abhängigkeit von der sozialen und ethnisch-kulturellen Herkunft oder des schulischen Beratungsprozesses) und die Verarbeitung des Übergangsprozesses durch Schüler und Eltern zu untersuchen. In Bezug auf verschiedene Schülermerkmale (emotionales Erleben und motivationale Bewältigung des Grundschulübergangs) zeigte sich, dass insgesamt Emotionen der Herausforderung gegenüber Emotionen der Bedrohung bei Schülern vor dem Übergang überwogen und dass Schüler mit einer Gymnasialempfehlung über ein geringeres Bedrohungserleben berichteten (Kurtz, Watermann, Klingebiel \& Szczesny, 2010), was die Ergebnisse von van Ophuysen (2006) und Sirsch (2006) bestätigte. Zudem konnte in der Studie von Watermann, Kurtz und Klingebiel (2010) nachgewiesen werden, dass sich der Bezugsgruppenwechsel negativ auf akademisches Selbstkonzept und intrinsische Motivation von Schülern, die auf ein Gymnasium wechselten, auswirkte. An dieser Schulform sanken Selbstkonzept und Motivation stärker als an den anderen Schulformen.

Die vorgestellten deutschsprachigen Studien verbindet, dass sie allesamt die Leistungsdifferenzierung des bundesdeutschen Schulsystems am Grundschulübergang in ihre Untersuchungen einbezogen haben. Dabei standen verschiedene motivationale, emotionale und kognitive Variablen im Zentrum der Studien, hingegen nahmen nur wenige der Untersuchungen Zielorientierungen, als einen bedeutsamen Teil der motivationaler Variablen, in den Blick. Aus diesem Grund soll der Fokus der vorliegenden Arbeit auf Zielorientierungen am Grundschulübergang liegen. Im Folgenden wird das Konstrukt ausführlicher dargestellt. 


\section{$2.2 \quad$ Zielorientierung}

\subsubsection{Definition}

Zielorientierungen (achievement goals) stellen ein weit erforschtes Konstrukt in der pädagogisch-psychologischen Forschung der letzten Jahrzehnte dar. Bereits in den 1970er Jahren entwickelten sich drei größere theoretische Ansätze in diesem Bereich: die Ansätze der Arbeitsgruppen um Carol Dweck (Dweck, 1986) und Carole Ames (Ames, 1984), sowie der aus der Entwicklungspsychologie stammende Ansatz der Arbeitsgruppe um John G. Nicholls (Nicholls, 1978, 1984). Oberflächlich betrachtet ähneln sich diese drei Ansätze sehr. Sie alle gehen von zwei Formen der Ziele aus: auf der einen Seite stehen die Aufgabe und der Kompetenzzuwachs im Vordergrund, auf der anderen Seite Wettbewerb und die eigenen Fähigkeiten. Bei genauerer Betrachtung der theoretischen Konzepte der jeweiligen Arbeitsgruppen können allerdings nur wenige Gemeinsamkeiten gefunden werden. Auch in der neueren Forschung zum Thema Zielorientierung besteht oftmals noch Uneinigkeit darüber, was genau unter diesem Konzept zu verstehen ist und wie die einzelnen Formen der Zielorientierung zu definieren sind.

In der vorliegenden Dissertation wird sich auf die folgende Definition von Zielorientierung bezogen: Zielorientierung ist eine ,future-focused cognitive representation that guides behavior to a competence-related end state that the individual is committed to either approach or avoid“ (Hulleman et al., 2010, S. 423). Sie geben Auskunft darüber, warum Personen in leistungsthematischen Situationen versuchen etwas zu erreichen (Schunk et al., 2008).

Sowohl in früheren als auch in aktuelleren Studien wurden bzw. werden Zielorientierungen als Personenmerkmale und/oder als aktuelle motivationale Zustände von Personen betrachtet (Köller, 1998, Schöne, 2007). In der vorliegenden Untersuchung werden Zielorientierungen als habituelle Merkmale mit kontextueller Sensitivität angesehen (Pintrich, 2000b). Personen streben demnach über einen längeren Zeitraum wiederholt in Lernsituationen bestimmte Ziele an, welche allerdings je nach Kontext variieren können.

Es lassen sich zwei grundlegende Dimensionen von Zielen unterscheiden, welche in den verschiedenen Zieltheorien (engl. goal theories) mit unterschiedlichen Begriffen belegt sind, sich aber dennoch auf vergleichbare Sachverhalte beziehen. Dweck (1986) und Dweck \& Leggett (1988) verwenden die Begriffe Lern- und Leistungsziele (learning goals vs. performance goals). Ames und Ames (1984) sprechen von Bewältigungs- und Leistungszielen (mastery goals vs. performance goals) und Nicholls $(1984,1992)$ von Aufgaben- und Ichori- 
entierung (task orientation vs. ego orientation). In der Bedeutung der verschiedenen Termini existieren zwar feine Differenzen, dennoch werden sie in den meisten Fällen synonym verwendet. Ihnen ist gemein, dass sie stets eine motivationale Orientierung, die zum einen auf die Verbesserung der eigenen Kompetenz und zum anderen auf die Darstellung der eigenen Kompetenz bzw. das Verschleiern fehlender Kompetenz gerichtet ist. Im Folgenden werden die Begriffe Lern- und Leistungsziel(-orientierung) verwendet, da sich diese Bezeichnungen in der pädagogisch-psychologischen Forschung des letzten Jahrzehnts durchgesetzt haben.

Bei Lernzielen stehen die Aufgabe an sich und das Streben nach Kompetenzzuwachs im Vordergrund. Für lernzielorientierte Schüler sind das Lernen, Verstehen, Entwickeln von Kompetenzen und Meistern von Aufgaben von zentraler Bedeutung. Bei Leistungszielen ist hingegen der soziale Vergleich der eigenen Leistungen vordergründig. Leistungszielorientierten Schülern ist es wichtig, welchen Eindruck die eigenen Fähigkeiten auf andere Schüler machen (Schunk et al., 2008). Dabei können zum einen das Bestreben nach Kompetenzdemonstration und die Zurschaustellung der eigenen Fähigkeiten und Leistungen (Annäherungsleistungsziele) zentral sein und zum anderen das Vermeiden von negativer Kompetenzbewertung und das Verbergen fehlenden Könnens und Wissens (Vermeidungsleistungsziele). Die Unterteilung in Annäherungs- und Vermeidungsleistungsziele (Elliot, 1997; Elliot \& Harackiewicz, 1996; Elliot \& McGregor, 2001) stellt eine wichtige Erweiterung des Zielorientierungskonzepts dar, welche bedeutend zur Klärung der oftmals inkonsistenten Befundlage der Leistungsziele beigetragen hat (Elliot, 1997, 1999).

Einige neuere Studien nehmen bei Lernzielen ebenfalls die Unterteilung in Annäherungs- und Vermeidungslernziele vor (Bong, 2009; Elliot, 1999; Linnenbrink \& Pintrich, 2000, 2002, Pintrich, 2000b). Unter einer Vermeidungslernzielorientierung wird hierbei zum einen das Vermeiden eines nicht maximalen Kompetenzzuwachses und zum anderen das Bestreben, Kompetenzverlust zu vermeiden, etwa Gelerntes nicht zu vergessen, verstanden. Der Forschungsstand dazu ist allerdings gering und es besteht Uneinigkeit über die grundsätzliche Existenz von Vermeidungslernzielen bei jungen Schülern (Grundschule, Sekundarstufe I), da bei diesen der Kompetenzzuwachs die Regel ist, nicht aber die Abnahme von Fähigkeiten (wie dies im Alter eher der Fall ist) (Schöne, 2007). Aus diesem Grund wird in der vorliegenden Arbeit ebenfalls auf diese Trennung verzichtet.

In einer aktuellen Studie von Elliot, Murayama und Pekrun (2011) wird zudem ein neuer Ansatz der Einteilung von Zielorientierungen untersucht $(3 \times 2$ achievement goal model). So weesen die Autoren in ihrer Untersuchung nach, dass sowohl task-based, self-based als auch other-based Zielorientierungen existieren. Diese Formen von Zielorientierungen un- 
terscheiden sich in ihrer Definition und Bewertung von Kompetenz. So beziehen sich taskbased Zielorientierungen auf den Lerngegenstand an sich, wohingegen bei self-based Zielorientierungen intrapersonale und bei other-based Zielorientierungen interpersonale Vergleiche im Vordergrund stehen. Zudem wird auch hier die Einteilung in eine Annäherungs- und eine Vermeidungskomponente vorgenommen, sodass sich insgesamt sechs Formen von Zielorientierungen ergeben: task-approach, task-avoidance, self-approach, self-avoidance, otherapproach und other-avoidance. In der bisherigen Forschung wurden task- und self-based Zielorientierungen nicht voneinander getrennt betrachtet, sondern gemeinsam unter dem Begriff Lernziele subsummiert. Da es sich bei dieser Untersuchung jedoch um eine aktuelle Studie handelt, wessen Annahmen bislang in keiner weiteren Studie repliziert wurden, wird sich in der vorliegenden Arbeit auf das trichotome Modell der Zielorientierungen mit der Einteilung in Lern-, Annäherungsleistungs- und Vermeidungsleistungsziele bezogen.

\subsubsection{Zielorientierungen und Selbstregulation}

Um erfolgreich lernen zu können und den steigenden Anforderungen der Schule und der Umwelt entsprechen zu können, ist die Fähigkeit, das eigene Lernen zu organisieren und $\mathrm{zu}$ regulieren, besonders wichtig. Unter Selbstregulation versteht man einen aktiven, konstruktiven Prozess, bei welchem sich der Schüler Ziele für das Lernen setzt und seine Kognition, Motivation und Verhalten überwacht, reguliert und kontrolliert, um diese Ziele zu erreichen (Pintrich, 2000b). Selbstreguliertes Lernen ist ein Prozess, bei welchem Gedanken, Gefühle und Handlungen auf die individuellen Ziele abgestimmt werden (Hasselhorn \& Labuhn, 2008). Es entwickelten sich zahlreiche Modelle aus einer Vielzahl von theoretischen Perspektiven. Zielorientierungen stellen dabei einen wichtigen Bestandteil des selbstregulierten Lernens dar. Sie sind im zyklischen Phasenmodell von Zimmerman (1998; Zimmerman \& Campillo, 2003) Teil der motivationalen Überzeugungen der Vorbereitungsphase. Auch in dem Rahmenmodell von Pintrich (2000b) stellen Zielorientierungen ein zentrales Konstrukt dar. Sie sind der Grund dafür, warum sich der Lernende mit bestimmten Aufgaben beschäftigt, sich besonders anstrengt oder warum er gute Noten bekommen möchte. In diesem Kontext hinterfragt Pintrich die Beziehung zwischen der Motivation, bzw. den Zielorientierungen und der Selbstregulation, wobei er die positive Bedeutung der Lernziele aus der Sicht der Selbstregulation betont. Im Gegensatz zu den Leistungszielen zeigen lernzielorientierte Schüler positivere Ergebnisse bezüglich der Strategienutzung während des Monitorings und der 
Kontrolle. Außerdem zeigen sie positivere Selbstwirksamkeitserwartungen und eine bessere Zeit- und Anstrengungseinteilung (Pintrich, 2000b).

Demnach stellt das Streben nach Lern- und Leistungszielen einen zentralen Aspekt des selbstregulierten Lernens dar, welcher analog zu dem Setzten von Zielen in den oben vorgestellten Modellen zur Selbstregulation ist.

\subsubsection{Zusammenhang mit verschiedenen Variablen}

\subsubsection{Motivationale, kognitive und behaviorale Variablen}

Generell konnte in der Forschung zum Thema Zielorientierung und dem Zusammenhang $\mathrm{zu}$ verschiedenen motivationalen, emotionalen und kognitiven Variablen in zahlreichen Untersuchungen gezeigt werden, dass ein positiver Zusammenhang zwischen Lernzielen und Variablen besteht, welche als besonders adaptiv für das Lern- und Leistungsverhalten gelten (Lemos, 1999). So zeigte sich, dass lernzielorientierte Schüler u.a. über hohe Selbstwirksamkeitserwartungen (Ames, 1992; Pintrich, 2000a), hohes Wohlbefinden (Ames, 1992), Selbstkonzept (z.B. Harackiewicz, Barron, Pintrich, Elliot \& Thrash, 2002) und intrinsische Motivation (Ames, 1992; Harackiewicz, Barron \& Elliot, 1998; Rawsthorne \& Elliot, 1999) verfügen. Außerdem wurde nachgewiesen, dass Schüler mit ausgeprägten Lernzielen einen günstigen Attributionsstil aufweisen (Ames, 1992; Dweck \& Leggett, 1988). Sie verbinden die erbrachte Leistung, den Erfolg bzw. Misserfolg verstärkt mit der dafür aufgebrachten Anstrengung. Des Weiteren weisen lernzielorientierte Schüler eine positive Einstellung zum Lernen auf, sie berichten häufiger über positive Affekte wie Freude und Zufriedenheit (Ames, 1992; Harackiewicz, Barron, Tauer, Carter \& Elliot, 2000; Harackiewicz, et al., 2002; Rawsthorne \& Elliot, 1999). Beim Lernen setzten sie vermehrt Tiefenverarbeitungsstrategien wie Elaborations- und Transformationsstrategien ein (Bandalos, Finney \& Geske, 2003; Pintrich, 1999; Wolter, Yu \& Pintrich, 1996) und zeigen adaptives Hilfesuchen (Karabenick, 2004; Linnenbrink, 2005; Ryan, Pintrich \& Midgley, 2001). Zudem bevorzugen sie herausfordernde Aufgaben, zeigen eine hohe Risikobereitschaft (Dweck, 2000; Dweck \& Leggett, 1988) und ein großes Interesse am Lerngegenstand (Spinath \& Schöne, 2003).

Im Gegensatz dazu sind die Befunde zum Zusammenhang von Leistungszielen und verschiedenen motivationalen, emotionalen und kognitiven Variablen weniger konsistent. Zunächst wurde in zahlreichen Untersuchungen angenommen, dass diese Form der Zielorien- 
tierung negativ mit verschiedenen Variablen des adaptiven Lernens zusammenhängt, wie zum Beispiel intrinsischer Motivation und Selbstwirksamkeitserwartung (Elliot \& Church, 1997). Allerdings wurden auch gegenteilige Ergebnisse gefunden, was auf eine fehlende Differenzierung nach Annäherungs- und Vermeidungsleistungszielen zurückgeführt wurde. So besteht in neueren Untersuchungen weitestgehend Einigkeit über die negativen Auswirkungen bzw. Zusammenhänge hoher Vermeidungsleistungsziele mit adaptivem Lernverhalten. Vermeidungsleistungszielorientierte Schüler verfügen über geringere Selbstwirksamkeitserwartungen (Middleton \& Midgley, 1997; Skaalvik, 1997), über eine geringere intrinsische Motivation (Rawsthorne \& Elliot, 1999) und über einen maladaptiven Attributionsstil (Ames, 1992; Dweck \& Leggett, 1988). Sie sehen Erfolg bzw. Misserfolg eher in ihren Fähigkeiten begründet. Misserfolg wird auf Inkompetenz zurückgeführt. Zudem wurden Verbindungen zu Bedrohungsgefühlen, Ängstlichkeit (Linnenbrink, 2005; Middleton \& Midgley, 1997; Skaalvik, 1997), einem geringerem Interesse am Lerngegenstand (Rawsthorne \& Elliot, 1999), der Nutzung von leistungshinderlichen Lernstrategien wie Oberflächenstrategien (Midgley \& Urdan, 2001), der Bevorzugung von leichten Aufgaben (Pintrich, 2000a) und einer geringeren Einschätzung der eigenen Kompetenzen (Elliot \& Sheldon, 1998) gefunden.

Leistungsziele hängen allerdings nicht zwingend mit negativen Konsequenzen zusammen. So haben sich Annäherungsleistungsziele als adaptiv beispielsweise in Bezug auf Anstrengungsbereitschaft (Elliot, \& McGregor \& Gable, 1999), Interesse, intrinsischer Motivation und Wertschätzung (Bong, 2001; Skaalvik, 1997; Wolters et al., 1996) erwiesen. Zu anderen Variablen wie der Nutzung von Lernstrategien (Middleton \& Midgley, 1997; Wolters et al., 1996) oder Selbstwirksamkeitserwartung (Anderman \& Midgley, 1997; Middleton \& Midgley, 1997) sind die Befunde hingegen uneinheitlich.

\subsubsection{Schulische Leistungen}

Zahlreiche Untersuchungen haben sich mit dem Zusammenhang zwischen Zielorientierungen und schulischen Leistungen beschäftigt. Oftmals haben sich hierbei Lernziele als leistungsförderlich erwiesen (Church, Elliot \& Gable, 2001; Diseth \& Kobbeltvedt, 2010; Elliot \& McGregor, 1999; Green \& Miller, 1996; Lau \& Nie, 2008; Yeo, Loft, Xiao \& Kiewitz, 2009). In einigen Studien wurden allerdings auch Null-Korrelationen gefunden (Brett \& VandeWalle, 1999; Senko \& Harackiewicz, 2005). Linnenbrink-Garcia, Tyson und Patall (2008) konnten in ihrem Review von mehr als 90 Studien zum Zusammenhang zwischen Zielorientierungen und schulischen Leistungen bei ca. 40 Prozent der Untersuchungen 
eine positive Verbindung zwischen Lernzielen und Leistungen und bei lediglich fünf Prozent eine negative Verbindung nachweisen. Das Zusammenhangsmuster scheint allerdings je nach Aufgabenform, Alter der untersuchten Personen und Art der Analyse zu variieren. So haben sich Lernziele als besonders adaptiv bei jungen Schülern (im Vergleich zu Studierenden) (Kaplan \& Maehr, 1999; Midgley \& Urdan, 1995; Roeser, Midgley \& Urdan, 1996; Ryan, Patrick \& Shim, 2005; Wentzel, 1993; Wolter et al., 1996) und in Lernsituationen erwiesen, in welchen konzeptuelles Lernen an Stelle von bloßem Auswendiglernen zentral ist (Pekrun, Elliot \& Meier, 2009). Bei Studierenden konnte hingegen konsistent kein Zusammenhang zu Leistungen nachgewiesen werden (Harackiewicz, Barron, Pintrich et al., 2002).

Ein gegenteiliges Ergebnis, also ein negativer Zusammenhang mit schulischen Leistungen, wurde oftmals für Vermeidungsleistungsziele gefunden (Church et al., 2001; Diseth \& Kobbeltvedt, 2010; Elliot \& McGregor, 2001; Finney, Pieper, \& Barron, 2004; Vansteenkiste et al., 2004; Wolters, 2004). Aber auch hier lassen sich einige Studien mit NullKorrelationen nennen (Sideridis, 2005; Tanaka \& Yamauchi, 2001).

Köller (1998) untersuchte den Zusammenhang zwischen Zielorientierungen und Schulleistung mit Hilfe curricular valider Tests bei Schülern der siebten Jahrgangsstufe im Längsschnitt und wies bei lernzielorientierten (aufgabenorientierten) Schülern höhere Lernraten nach als bei leistungszielorientierten (ichorientierten) Schülern.

Bei Annäherungsleistungszielen sind die Befunde weniger einheitlich. Wie auch schon beim Zusammenhang mit einigen adaptiven Kognitionen und Verhaltensweisen, zeigt diese Form der Zielorientierung oftmals eine positive Verbindung mit schulischen Leistungen (Elliot \& Church, 1997; Elliot et al. , 1999; Harackiewicz et al., 1997; Harackiewicz, Barron \& Elliot, 1998; Harackiewicz et al., 2000; Spinath, Stiensmeiser-Pelster, Schöne \& Dickhäuser, 2002; Urdan, 2004; Null-Korrelationen siehe: Lee, Sheldon \& Turban, 2003; Pajares \& Valiante, 2001). Auch bei den Annäherungsleistungszielen konnten Linnenbrink-Garcia, Tyson und Patall (2008) in ihrem Review von mehr als 90 Studien bei 40 Prozent der betrachteten Untersuchungen einen positiven Zusammenhang mit schulischen Leistungen finden und bei fünf Prozent einen negativen. Diese Form der Zielorientierung scheint demnach sowohl positive (mit der Annäherungskomponente verbundene Aspekte wie z.B. Anstrengung, Persistenz) als auch negative Elemente (Fokus auf externe Vergleiche) zu vereinen. Die positiven Elemente werden oftmals von den negativen ausgeglichen. So haben Midgley, Kaplan und Middleton (2001) in ihrem Review zusammengefasst, dass sich Annäherungsleistungsziele unter bestimmten Bedingungen (in kompetitiven Lernumwelten) und für bestimmte Schüler (Jungen, ältere Schüler) adaptiv auswirken. Bei jungen Schülern scheint diese Form der Ziel- 
orientierung hingegen keinen Einfluss auf die schulischen Leistungen zu nehmen (Pajares, Britner \& Valiante, 2000; Pajares \& Valiante, 2001) oder sogar potentiell maladaptiv zu sein (Middleton, Kaplan \& Midgley, 2004).

Der Forschungsstand zum Zusammenhang von Zielorientierungen und verschiedenen motivationalen, emotionalen und kognitiven Variablen belegt demnach, dass Lern- und Leistungsziele unterschiedliche Ergebnisvariablen begünstigen. So scheinen sich Lernziele hauptsächlich positiv auf das Ausmaß an Interesse, intrinsischer Motivation, Anstrengung und Ausdauer auszuwirken (Barron \& Harackiewicz, 2001; Elliot et al., 1999; Harackiewicz, Barron, Tauer \& Elliot, 2002), wohingegen Annäherungsleistungsziele besonders bei älteren Schülern und Studierenden leistungsfördernd sind (Harackiewicz, Barron, Pintrich et al., 2002; Harackiewicz, Durik, Barron, Linnenbrink \& Tauer, 2008; Hulleman, Durik, Schweigert \& Harackiewicz, 2008). Auch wenn in einigen Untersuchungen kein direkter Zusammenhang zwischen Lernzielen und schulischen Leistungen nachgewiesen werden konnte, können dennoch indirekte Effekte auf die Leistung auftreten, beispielsweise vermittelt über Anstrengung, Persistenz und positive Emotionen (Daniels et al., 2008; Wild \& Möller, 2009). Die Betrachtung von Moderator- und/oder Mediatorvariablen könnte zur Klärung der uneinheitlichen Forschungsergebnisse demnach nicht nur bei Lernzielen, sondern auch bei Leistungszielen einen Beitrag leisten (Pintrich, 2000a).

Die inkonsistente Befundlage zum Zusammenhang zwischen Zielorientierungen und Leistungen lassen sich zum einen mit Differenzen zwischen experimentellen und Feldstudien und zu anderen mit Differenzen zwischen Untersuchungen an Schülern und Studierenden erklären (Midgley et al., 2001; Utman, 1997). Des Weiteren bestehen in den vorgestellten Untersuchungen Diskrepanzen hinsichtlich der Operationalisierung der Zielorientierungen (Grant \& Dweck, 2003; Harackiewicz, Barron, Pintrich et al., 2002). So haben Hulleman et al. (2010) nach Sichtung von 243 Studien, welche Zielorientierungen erhoben haben (bis zum Jahr 2006 publizierte als auch unpublizierte Studien), nachweisen können, dass Differenzen, welche das Konzept und die Operationalisierung von Zielorientierungen betreffen, existieren und dass diese Differenzen die Beziehung zu verschiedenen Variablen beeinflussen können: „Clearly, achievement researchers are using the same label for different constructs“ (Hulleman et al., 2010, S. 441). Die Autoren haben verschiedene Komponenten der jeweiligen Zielorientierungen definiert, welche sich auf unterschiedliche Konstrukte beziehen. So konnten sie eine Vielzahl von verschiedenen Komponenten der Lernziele (u.a. Interesse, Neugierde, Verbesserung, Herausforderung) identifizieren, sowie drei bzw. vier verschiedene 
Komponenten der Annäherungsleistungsziele und der Vermeidungsleistungsziele (auf die eigene Erscheinung bezogene, normative, evaluative Komponenten). Diese verschiedenen Komponenten hängen unterschiedlich u.a. mit schulischen Leistungen zusammen. So konnten für die Annäherungsleistungsziele bei Items, welche unter die normative Komponente zusammengefasst werden konnten (bessere Leistungen als andere zu erbringen), positive Korrelationen zu Leistungen nachgewiesen werden, wohingegen bei Items, welche eine auf die eigenen Erscheinung bezogene oder evaluative Komponente enthielten, negative Korrelationen gefunden wurden (Hulleman et al., 2010).

Des Weiteren haben Hulleman et al. (2010) festgestellt, dass bei einem nicht unbedeutenden Teil der untersuchten Studien Items eingesetzt wurden, welche sich nicht explizit auf Zielorientierungen bezogen haben, sondern eher allgemein formuliert waren, wie beispielsweise „I do my work because I'm interested in it.” (S. 423). Dies war bei 52 Prozent der Lernzielitems, bei 29 Prozent der Annäherungsleistungszielitems und bei 64 Prozent der Vermeidungsleistungszielitems der untersuchten Studien der Fall. Diese Items haben stattdessen die Präferenz für Herausforderungen und das Interesse allgemein erhoben. Waren die Items der Lernziele beispielsweise so formuliert, dass sie sich auf bestimmte Zielorientierungen bezogen (z.B. „An important reason why I do my class work is because I like to learn new things.”), ergaben sich keinerlei Zusammenhänge zu den Leistungen. Beinhalteten die Items hingegen keine „goal-relevant language“ („I like learning new things form physical education, even if I make mistakes”), zeigten sich positive Korrelationen zu den Leistungen.

Des Weiteren fanden die Autoren Variabilität in der Messung der einzelnen Komponenten sowohl zwischen als auch innerhalb der jeweiligen Untersuchungen. Demzufolge ist es nicht verwunderlich, dass die Befundlage zu diesem Thema inkonsistent ist. Der Zusammenhang zwischen Zielorientierungen und verschiedenen Variablen hängt demnach entscheidend davon ab, wie Zielorientierungen definiert und operationalisiert werden. Einschränkend muss jedoch angemerkt werden, dass von den 243 Studien aus der Metaanalyse lediglich 20 Untersuchungen an Grundschulen stattfanden. Ein Großteil der Studien fand stattdessen mit Studierenden statt. Die berichteten Befunde lassen sich demnach nicht ohne Weiteres auf jüngere Schüler übertragen.

Wie die Metaanalyse von Hulleman et al. (2010) verdeutlich hat, wurde nur ein sehr geringer Anteil der bisherigen Untersuchungen zu Zielorientierungen an Schülern im Grundschulbereich durchgeführt. Das Zusammenhangsmuster zwischen Zielorientierungen und schulischen Leistungen scheint allerdings vom Alter der untersuchten Schüler abhängig zu sein und sich im Laufe der Schulzeit zu verändern (Bong, 2009). In ihrer Untersuchung an 1 
196 Koreanischen Schülern von Klassenstufe eins bis neun konnte Bong keine Korrelation zwischen Zielorientierungen und schulischen Leistungen bei sehr jungen Schülern (1./2. Klassenstufe) finden. Erst bei etwas älteren Schülern ließen sich ein geringer positiver Zusammenhang zwischen Lernzielen und ein geringer negativer Zusammenhang zwischen Vermeidungsleistungszielen und Schulleistungen feststellen. Ab Klassenstufe fünf zeigten auch die Annäherungsleistungsziele positive Korrelationen zu den Schulleistungen.

In der Studie von Lau und Nie (2008) konnte ein geringer positiver Zusammenhang der Lernziele $(r=.19, p<.01)$ sowie ein geringer negativer Zusammenhang der Vermeidungsleistungsziele $(r=-.27, p<.01)$, jedoch kein Zusammenhang der Annäherungsleistungsziele mit den schulischen Noten in der Grundschule (elementary school, fünfte Klassenstufe) nachgewiesen werden. Einen ähnlichen Befund konnten auch Anderman und Anderman (1999) finden. In ihrer Untersuchung hingen Leistungsziele ebenfalls gering negativ mit den Noten zusammen $(r=-.13, p<.01)$. Andere Untersuchungen konnten hingegen keinen Zusammenhang zwischen Zielorientierungen und schulischen Leistungen in der Grundschule nachweisen (z.B. Anderman \& Midgley, 1997; Finsterwald, 2006).

Ähnlich wie bei Bong (2009) stellten sich auch in anderen Untersuchungen (z.B. Midgley et al.; 2001) Annäherungsleistungsziele als leistungsförderlich bei älteren Schülern oder Studierenden heraus, wohingegen sich Lernziele eher bei jüngeren Schülern adaptiv erwiesen (Pekrun et al.; 2009). Shim, Ryan und Anderson (2008) kamen zu dem Schluss, dass „the consequences of achievement goals may change as students mature and move through the educational system“ (S. 656). Mit dem Übergang von der Grundschule zur weiterführenden Schule ändert sich besonders in Deutschland der Schulkontext erheblich, welcher mit steigenden Leistungsanforderungen verbunden ist. Lernziele könnten in diesen kompetitiveren Lernkontexten weniger adaptiv in Bezug auf schulische Leistungen sein als Annäherungsleistungsziele.

\subsubsection{Vermittelnde Variablen}

In der Literatur wird der Zusammenhang zwischen Zielorientierungen und Schulleistungen vor allem mit dem Einsatz bestimmter Lernstrategien begründet (z.B. Köller, 1998). So wird angenommen, dass hohe Lernziele mit der aktiven Nutzung von Tiefenverarbeitungsstrategien einhergehen, wohingegen hohe Leistungsziele (nicht differenziert nach Annäherungs- und Vermeidungsleistungsziele) verstärkt mit der Anwendung von Oberflächenstrategien verbunden sind (u.a. Bouffard, Boisvert, Vazeau \& Larouche, 1995). 
Weitere Variablen, in denen sich Unterschiede in Abhängigkeit der Zielorientierung zeigen, sind beispielsweise Emotionen. Pekrun und Mitarbeiter betonen die Rolle von Zielorientierungen für Emotionen beim Lernen, wobei Lernziele mit positiven Emotionen (z.B. Freude) und Vermeidungsleistungsziele mit negativen Emotionen (z.B. Leistungsangst) in Verbindung gebracht werden (Pekrun, Elliot \& Maier, 2006).

Des Weiteren haben sich Unterschiede in den Attributionsstilen der Schüler in Abhängigkeit von der jeweiligen Zielorientierung gezeigt. Schüler mit hohen Leistungszielen führen ihre eigene Leistung vermehrt auf die eigene Fähigkeit zurück, wohingegen Schüler mit hohen Lernzielen oftmals die investierte Anstrengung als Ursache für die eigene Leistung betrachten (Ames, 1984). Da die intrinsische Motivation (Elliot \& Church, 1997; Meece, Blumenfeld \& Hoyle, 1988) und das Interesse am Lerngegenstand (Harackiewicz et al., 1997) bei lernzielorientierten Schülern oftmals ausgeprägter ist, könnte davon ausgegangen werden, dass sich diese Schüler verstärkt aktiv und kognitiv mit Aufgaben beschäftigen, über eine höhere Persistenz verfügen sowie tiefere Informationsverarbeitungsprozesse anwenden als leistungszielorientierte Schüler (Ames \& Archer, 1988; Miller, Behrens, Greene \& Newman, 1993; Nolen, 1988). Zudem zeigen sie bei auftretenden Schwierigkeiten eher hilfesuchendes Verhalten (Butler \& Neuman, 1995).

\subsubsection{Einfluss der Lernumwelt}

Welche Bedeutung die Lernumwelt für den Zusammenhang von Zielorientierungen zu verschiedenen motivationalen, emotionalen und kognitiven Merkmalen hat, wurde bislang nur wenig untersucht. In Studien, in denen unterschiedliche Charakteristika der Lernumwelt bei der Untersuchung von Zielorientierungen betrachtet wurden, bezogen sich hauptsächlich auf ältere Schüler bzw. auf Studierende (z.B. Harackiewicz et al., 1997). Midgley, Kaplan \& Middleton (2001) kamen nach Sichtung zahlreicher Untersuchungen zum Schluss, dass sich die Konsequenzen von Zielorientierungen im Laufe der Schulzeit ändern. Lernziele verlieren demnach im Laufe der Schulzeit ihre leistungsförderliche Wirkung, wohingegen der Zusammenhang zwischen Leistungszielen und Schulleistungen positiv und bedeutsamer wird. So sind Lernziele insbesondere bei sehr jungen Schülern (Grundschule) adaptiv und wirken sich positiv auf schulische Leistungen aus (Pekrun et al., 2009). Im Laufe der Schulzeit (weiterführenden Schule, Universität) wird dieser positive Zusammenhang jedoch immer kleiner bzw. verschwindet. Anders soll es sich bei den Annäherungsleistungszielen verhalten. bei 
sehr jungen Schülern wirken sich diese eher negativ auf die Schulleistungen aus ${ }^{7}$ (z.B. Anderman \& Anderman, 1999), im Laufe der Schulzeit bzw. auch darüber hinaus (Universität, College) werden sie jedoch adaptiver, was bedeutet, dass der Zusammenhang zu den Leistungen positiv wird (Harackiewicz et al., 1997).

Middleton, Kaplan und Midgley (2004) argumentieren, dass Annäherungsleistungsziele eher maladaptiv bei jungen Schülern sind, da die Lernumwelt an Grund- bzw. weiterführenden Schulen weniger kompetitiv ist als an der Universität (Middleton \& Midgley, 1997). Auch Harackiewicz et al. (1997) kommen zu einer ähnlichen Schlussfolgerung. In ihrer Untersuchung an undergraduates konnte ein positiver Zusammenhang zwischen Leistungszielen und Kursendnoten nachgewiesen werden. Sie argumentieren, dass dieser positive Zusammenhang dadurch entstanden ist, dass die Lernumwelt an colleges als kompetitiv und leistungsorientiert charakterisiert werden kann, was Leistungsziele adaptiver erscheinen lässt.

\subsubsection{Entwicklung von Zielorientierungen}

\subsubsection{Allgemein}

Um die Entwicklung von Zielorientierungen zu erklären, werden zwei Ansätze herangezogen. Zum einen wird Nicholls Theorie von Anstrengung und Begabung (1984, 1990) betrachtet und zum anderen Dwecks Implizite Theorie über die Modifizierbarkeit von Persönlichkeitseigenschaften (Dweck, 1986; Dweck \& Leggett, 1988). Beiden Theorien ist gemein, dass sie die Veränderungen im Verständnis von Fähigkeit bzw. Begabung zur Erklärung der Entwicklung von Zielorientierungen betrachten.

Der entwicklungspsychologische Ansatz von Nicholls (1984, 1990) vertritt die Annahme, dass sich die individuellen Konzepte des Fähigkeitsbegriffs mit zunehmendem Alter verändern und diese Konzepte Einfluss auf die motivationale Entwicklung nehmen. Bis zum Ende der Grundschulzeit verfügen die Schüler demnach noch über ein undifferenziertes Fähigkeitskonzept, sie unterscheiden nicht zwischen Anstrengung und Fähigkeit und sind der Ansicht, dass Personen, die sich mehr anstrengen, fähiger sind. Die Schüler verfügen zu diesem Zeitpunkt über eine individuelle Bezugsnormorientierung. Per Definition sind Kinder in dieser Entwicklungsphase lernzielorientiert (Köller, 1998; Schwinger \& Wild, 2006). Im Laufe ihrer kognitiven Entwicklung bildet sich allmählich ein differenziertes Fähigkeitskonzept heraus, sodass Kinder zunehmend zwischen Fähigkeit und Anstrengung unterscheiden (Ruble

\footnotetext{
${ }^{7}$ Wie gezeigt werden konnte, ist der Forschungsstand dazu allerdings inkonsistent.
} 
\& Frey, 1991). Im Alter von ungefähr sieben bis neun Jahren sind sie demnach in der Lage ihre Leistungen mit denen anderer zu vergleichen und zu bewerten. Dieser entwicklungspsychologisch bedingte Anstieg der Leistungsziele wurde allerdings bisher nicht empirisch belegt. Nicholls lässt ebenfalls offen, wann dieser Differenzierungsprozess einsetzt und ob er irreversibel ist.

In der Arbeitsgruppe um Dweck und Leggett (1988) wurden als zentrale Ursachen für die Entwicklung von Zielorientierungen naive Theorien über die Veränderbarkeit von Intelligenz herangezogen. So haben junge Kinder oftmals die Vorstellung, dass Intelligenz veränderbar sei, was einer incremental theory gleichkommt. Diese Kinder sollten eher Lernziele herausbilden, wohingegen Kinder, welche die Vorstellung haben, dass Intelligenz etwas Unveränderbares ist (entity theory), eher Leistungsziele entwickeln sollten. Dweck und Leggett (1988) konnten diese Annahmen in einer Fragebogenstudie weitestgehend bestätigen. Ein sehr großer Teil der Probanden (82\%), die der entity theory anhingen, entwickelten Leistungsziele, nur 18 Prozent Lernziele. Bei Personen mit einer incremental theory entwickelten 61 Prozent Lernziele und 39 Prozent Leistungsziele.

Ausgehend von den vorgestellten Ansätzen zur Entwicklung der Zielorientierung ist anzunehmen, dass Grundschüler überwiegend lernzielorientiert sind und erst im Laufe der kognitiven Entwicklung die Leistungszielorientierung zunimmt. Viele Studien, die sich mit Zielorientierungen in der Grundschule beschäftigten, konnten die Annahmen von Nicholls bestätigen. Sie fanden tatsächlich hohe Lernziele in der Grundschule (Anderman \& Anderman, 1999; Anderman \& Midgley, 1997; Bong, 2009; Finsterwald, 2006; Schwinger \& Wild, 2006; Shim et al., 2008). In einigen der Untersuchungen kam es im weiteren Verlauf zu einer Abnahme der Lernziele (Anderman \& Anderman, 1999; Anderman \& Midgley, 1997) und zu einer Zunahme der Leistungsziele (Anderman \& Anderman, 1999). Schwinger und Wild (2006) untersuchten die Zielprofile der Schüler von der dritten bis zur fünften Klassenstufe und fanden ein eher instabiles Zielprofil am Ende der Grundschulzeit vor.

Insgesamt scheinen Zielorientierungen bei Schülern im Grundschulalter jedoch weniger differenziert zu sein als bei älteren Schülern. So konnte Bong (2001) in ihrer Untersuchung an koreanischen Schülern der Sekundarstufe nachweisen, dass Zielorientierungen von jüngeren Schülern (middle school) höher miteinander korrelierten als Zielorientierungen von älteren Schülern (high school). Ähnliche Befunde konnten auch bei Grundschülern und Studierenden gefunden werden (Ross, Shannon, Salisbury-Glennon \& Guerino, 2002). Der Differenzierungsgrad der Zielorientierungen bzw. der motivationalen Orientierungen scheint bei 
jungen Schülern noch wenig ausgeprägt zu sein und sich erst mit zunehmendem Alter zu erhöhen (Marsh, Craven \& Debus, 1991).

\subsubsection{Am Übergang zur Sekundarstufe I}

Der Forschungsstand zur Entwicklung von Zielorientierungen allgemein ist sehr umfangreich (z. B. Ames \& Archer, 1988; Anderman, Austin \& Johnson, 2002; Köller, 1998), vergleichsweise wenige Untersuchungen lassen sich hingegen zur Entwicklung von Zielorientierungen am Übergang von der Grundschule in die Sekundarstufe I finden. Besonders im deutschsprachigen Raum ist die Forschungslage zu diesem Thema nicht befriedigend. Studien aus dem anglo-amerikanischen Raum (z.B. Anderman \& Anderman, 1999; Anderman et al., 2002; Anderman \& Midgley, 2004) belegen ein Absinken der Lernziele und ein Ansteigen der Leistungsziele beim Übergang von der Grundschule (elementary school) zur weiterführenden Schule (middle school). In einer anderen Studie von Anderman, Maehr und Midgley (1999) wurde der Übergang von der fünften in die sechste Klassenstufe untersucht und ebenfalls eine Verringerung der Lernziele sowie eine schulfach- und geschlechtsabhängige Entwicklung der Leistungsziele festgestellt. Auch Middleton et al. (2004) untersuchten die Entwicklung bzw. Stabilität von Zielorientierungen am Übergang zu der weiterführenden Schule. Sie wiesen eine moderate Stabilität der Lern- und Leistungsziele über die Zeit (sechste bis siebte Klassenstufe) nach. In anderen Untersuchungen (z.B. Bong, 2009; Shim et al., 2008) konnte hingegen eine Abnahme sowohl von Lern- als auch von Leistungszielen am Grundschulübergang belegen.

Da sich der Übergang von der Grundschule auf die weiterführende Schule im angloamerikanischen Raum in den meisten Staaten erst nach der sechsten Klassenstufe vollzieht, lassen sich die genannten Studien nicht ohne weiteres auf das deutsche Schulsystem übertragen, da hier der Schulwechsel schon nach der vierten Klassenstufe stattfindet. Die Forschung zur Entwicklung von Zielorientierungen am Grundschulübergang im deutschsprachigen Raum ist allerdings weniger umfangreich. Es lassen sich nur wenige Studien hierzu finden. Schwinger und Wild (2006) befassten sich mit der Entwicklung der Zielorientierungen von der dritten bis zur fünften Klassenstufe im Fach Mathematik. Die Autoren konnten den oben postulierten Trend zu einer vermehrten Leistungszielorientierung nach dem Schulwechsel nicht bestätigen. Insgesamt wurde für die Mehrheit der untersuchten Schüler ein eher instabiles Zielprofil am Ende der Grundschulzeit bzw. zu Beginn der Sekundarstufe I nachgewiesen. Allerdings konnte keine erhöhte Fluktuation in den Zielprofilen in der Zeit nach dem Über- 
gang festgestellt werden, sodass nicht von einem übergangsbedingten Effekt auf die Zielorientierungen ausgegangen wurde.

Finsterwald (2006) untersuchte die Bedingungsfaktoren eines erfolgreichen Übergangs auf weiterführende Schulen $(N=184)$ zu drei Messzeitpunkten (Ende Klassenstufe vier, Mitte und Ende Klassenstufe fünf) und konnte dabei ein Absinken aller drei Formen der Zielorientierungen nachweisen.

Einschränkend muss angemerkt werden, dass die vorgestellten Untersuchungen größtenteils mit Schülern im Alter ab ca. 12 Jahren (fünfte Klassenstufe) durchgeführt wurden. Lediglich die Studien von Schwinger und Wild (2006) und Finsterwald (2006) befassten sich mit jüngeren Schülern (dritte/ vierte Klassenstufe), allerdings fanden hier die Untersuchungen an keiner repräsentativen Stichprobe und im Fall von Schwinger und Wild (2006) zudem domänenspezifisch statt.

\subsubsection{Forschungsdesiderat hinsichtlich der Zielorientierungen am Grundschulübergang}

Die vorgestellten Studien machen deutlich, dass es eine Reihe an Untersuchungen zum generellen Grundschulübergang gibt. Zudem existieren zahlreiche Studien zur Entwicklung von Zielorientierungen und deren Zusammenhang zu motivationalen, emotionalen und kognitiven Merkmalen. Allerdings besteht großer Bedarf an Untersuchungen, welche die Entwicklung der Zielorientierungen am Grundschulübergang zu den verschiedenen Schulformen betrachten. Da in vielen Studien ein Zusammenhang zwischen Zielorientierungen und verschiedenen lern- und leistungsbezogenen Merkmalen nachgewiesen werden konnte und sich in vielen Fällen eine leistungsförderliche Wirkung vor allem der Lernziele gezeigt hat, ist davon auszugehen, dass Zielorientierungen für den schulischen Erfolg eine bedeutsame Rolle spielen. Ob sie allerdings bereits am Grundschulübergang ein wichtiger Faktor sind, welcher sich möglicherweise begünstigend auf die Bewältigung des Übergangs auswirkt, wurde bis dato nicht in ausreichendem Maße erforscht.

Da ein Großteil der Studien zum Thema Zielorientierung an college-Studenten im USamerikanischen Raum durchgeführt wurde und es nur vergleichsweise wenige Untersuchungen an jüngeren Schülern gibt - insbesondere an Grundschülern im deutschsprachigen Raum ist bislang unklar, ob sich die in Studien an älteren Schülern nachgewiesenen Zusammenhangsmuster der Zielorientierungen bereits bei Grundschülern nachweisen lassen. Wie gezeigt werden konnte, ist die Befundlage zum Zusammenhang zwischen Zielorientierungen und verschiedenen motivationalen, emotionalen und kognitiven Merkmalen insbesondere bei Schü- 
lern aus dem Grund- bzw. Sekundarschulbereich inkonsistent. Es bedarf demnach einer detaillierten und strukturierten Betrachtung dessen.

Dabei stellt die Untersuchung von Zielorientierungen an einer repräsentativen Stichprobe ein weiteres Forschungsdesiderat dar. Meist bezogen sich die vorgestellten Studien lediglich auf regional begrenzte Stichproben, welche wenig generalisierbar sind.

Ungeklärt ist bislang auch, ob Zielorientierungen als Teil motivationaler Orientierungen ähnlich wie die Motivation am Grundschulübergang absinken und inwieweit die Passung zwischen den steigenden Bedürfnissen der Schüler nach Selbstwirksamkeit und den veränderten Merkmalen des Klassenraums nach dem Grundschulübergang die Entwicklung von Zielorientierungen beeinflusst. Bis dato sind keine Untersuchungen diesbezüglich im deutschsprachigen Raum bekannt. Zudem ist bislang offen, welche Rolle Zielorientierungen am Grundschulübergang zu den verschiedenen Schulformen des bundesdeutschen dreigliedrigen Schulwesens zukommt. Somit soll ein besonderes Augenmerk auf die differenzielle Entwicklung von Zielorientierungen an den verschiedenen Schulformen gelegt werden. Mit dem Wechsel der Bezugsgruppe nach dem Übergang stellt sich die Frage, inwieweit diese die Entwicklung der Zielorientierungen - ähnlich wie dies oftmals beim Fähigkeitsselbstkonzept, dem Interesse oder der intrinsischen Motivation nachgewiesen werden konnte - beeinflusst. Dazu werden im Folgenden die Effekte der Bezugsgruppe vorgestellt. 


\subsection{Bezugsgruppeneffekte}

Mit den besonderen Merkmalen des leistungsdifferenzierenden bundesdeutschen Schulsystems stellt sich in der vorliegenden Arbeit die Frage, inwieweit die Merkmale dieses Schulsystems einen Einfluss auf die Entwicklung von Zielorientierungen und das Zusammenhangsmuster dieser mit verschiedenen Variablen schulischen Lernens ausüben. Mit dem Wechsel von der Grundschule auf die weiterführende Schule geht auch ein Wechsel der Bezugsgruppe einher. Der Einfluss der Bezugsgruppe auf die Entwicklung beispielsweise des Fähigkeitsselbstkonzepts, des Interesses, der intrinsischen Motivation oder Bildungsaspirationen konnte in zahlreichen Studien nachgewiesen werden (z.B. Chiu et al., 2008; Marsh et al., 2001; Marsh, Kong \& Hau, 2000; Marsh \& O’Mara, 2010; Trautwein, Lüdtke, Marsh et al., 2006). Bezugsgruppeneffekte basieren auf sozialen Vergleichsprozessen (frame-of-reference effects; Marsh, 1986). Schüler vergleichen die eigenen Leistungen mit denen ihrer Mitschüler und generieren ihre selbstbezogenen Einschätzungen (z.B. Selbstkonzept, Selbstwert) und Einstellungen (z.B. Motivation, Interesse) aus diesem Vergleich. Der Einfluss der Bezugsgruppe kann sich dabei auf unterschiedliche Weise auswirken. Im Folgenden soll zum einen auf den Big-Fish-Little-Pond-Effekt sowie den Basking-In-Reflected-Glory-Effekt eingegangen werden.

\subsubsection{Der Big-Fish-Little-Pond-Effekt}

Beim Big-Fish-Little-Pond-Effekt (BFLPE; Marsh, 1987, 1990; Marsh \& Craven, 1997; Marsh et al., 2000) wird davon ausgegangen, dass ein negativer Zusammenhang zwischen der mittleren Leistungsstärke einer Lerngruppe und dem Selbstkonzept der einzelnen Schüler besteht. Dieser Zusammenhang kommt durch soziale Vergleichsprozesse der Schüler untereinander zustande. Dabei kann das Resultat dieser Vergleichsprozesse für Schüler mit gleicher schulischer Leistung unterschiedlich ausfallen. So wird das Selbstkonzept eines Schülers mit mittlerer Leistungsstärke in einer leistungsstarken Lerngruppe (wie beispielsweise am Gymnasium) negativ beeinflusst werden, da die eigene Leistung des Schülers wahrscheinlich eher am unteren Ende der Leistungsverteilung der Lerngruppe sein wird. Dieser Schüler wird vermutlich die Erfahrung machen, zu den leistungsschwächsten Schülern der Lerngruppe zugehören. Umgekehrt verhält es sich mit einem Schüler mittlerer Leistungsstärke in leistungsschwachen Lerngruppen. Da sich dieser Schüler eher am oberen Ende der Leis- 
tungsverteilung befinden wird und zu den leistungsstärksten Schülern der Lerngruppe gehört, wird dessen Selbstkonzept gestärkt.

Dieser Kontrasteffekt konnte im deutschen Schulsystem wiederholt nachgewiesen werden (z.B. Aust et al., 2010; Köller, 2004; Schwarzer, Lange \& Jerusalem, 1982; Watermann et al., 2010; für die Grundschule vgl. Zeinz \& Köller, 2006). Die Forschergruppe um Trautwein, Lüdtke und Köller konnte in ihrer PISA-Erweiterungsstudie den negativen Bezugsgruppeneffekt in Hinblick auf Selbstkonzept, Selbstwert, Interesse und Motivation bei Schülern der neunten Klassenstufen in Mathematik belegen (z.B. Trautwein, Köller, Lüdtke \& Baumert, 2005; Trautwein, Lüdtke, Marsh et al., 2006; Trautwein, Lüdtke, Köller \& Baumert, 2006). Am Grundschulübergang wurde der BFLPE erstmals von Schwarzer et al. (1982) gezeigt. Das Fähigkeitsselbstkonzept von Schülern, die auf das Gymnasium wechselten, war beim Schulübergang höher als bei Schülern, die auf die Hauptschule wechselten. Innerhalb der ersten Monate an der weiterführenden Schule verringerte sich dieser Unterschied allerdings, sodass kaum noch Unterschiede im Selbstkonzept der Schüler bestanden. Daraus lässt sich schließen, dass sich die Schüler innerhalb einer eng umgrenzten Lerngruppe vergleichen und weniger darüber hinaus mit der gesamten Altersgruppe.

Zu einem ähnlichen Ergebnis kam Buff (1991) in seiner Untersuchung des Fähigkeitsselbstkonzepts am Grundschulübergang. Auch er konnte ein Absinken des Selbstkonzepts bei Schülern, welche auf die höhere Schulform wechselten (zweigliedrige Sekundarstufe I im Schweizer Kanton Bern), und ein Ansteigen bei Schülern der niedrigeren Schulform nachweisen. Zudem konnte Buff belegen, dass bereits vor dem Grundschulübergang Unterschiede in der Ausprägung des Fähigkeitsselbstkonzepts bei den Schülern bestanden. So verfügten künftige Schüler der höheren Schulform über ein ausgeprägteres Selbstkonzept als künftige Schüler der niedrigeren Schulform.

Ebenso konnten Valtin und Wagner (2004) in ihrer Längsschnittstudie am Grundschulübergang nachweisen, dass verglichen mit künftigen Hauptschülern künftige Gymnasiasten in der vierten Klassenstufe über - im Zusammenhang mit schulischem Lernen - positivere Schülermerkmale verfügten (höheres Fähigkeitsselbstkonzept und Selbstwertgefühl, günstigere Misserfolgsattributionen, weniger Leistungsangst). Nach dem Wechsel auf die weiterführende Schule glichen sich diese Werte jedoch an, was für den BFLPE spricht.

Aust, Watermann und Grube (2010) nahmen ebenfalls den Grundschulübergang in Bezug auf Bezugsgruppeneffekten beim Fähigkeitsselbstkonzept von Schülern in den Blick. Ergebnis ihrer mikrogenetischen Studie war, dass der BFLPE bereits kurze Zeit nach dem Übergang auf das Gymnasium auftrat, es konnte hingegen kein Bezugsgruppeneffekt an der 
Gesamtschule nachgewiesen werden. Zudem kam es in den leistungsstarken Schwerpunktklassen zu einer Abnahme des allgemeinen Selbstkonzepts, nicht jedoch des fachspezifischen Selbstkonzepts in Mathematik und Deutsch (Aust, Watermann \& Grube, 2009).

Des Weiteren wiesen Watermann, Kurtz und Klingebiel (2010) einen negativen Bezugsgruppeneffekt sowohl beim akademischen Selbstkonzept als auch bei der intrinsischen Motivation bei Schülern nach, die auf ein Gymnasium wechselten, wobei sich die Geschwindigkeit des Eintretens des BFLPEs deutlich zwischen den beiden Merkmalen unterschied. So nahm das Selbstkonzept der Schüler wesentlich stärker im ersten Halbjahr an der weiterführenden Schule ab als die intrinsische Motivation.

\subsubsection{Der Basking-In-Reflected-Glory-Effekt}

Ein weiterer Bezugsgruppeneffekt stellt der Basking-In-Reflected-Glory-Effekt dar (BIRGE; Cialdini et al., 1976; Marsh, 1984; Marsh et al., 2000). Dieser Assimilationseffekt zeigt in leistungsstarken Lerngruppen dem BFLPE entgegengesetzte Auswirkungen auf beispielsweise das Selbstkonzept der Schüler. Das positive Ansehen der Gruppe wirkt sich positiv auf das Selbstkonzept der Schüler aus. In leistungsstarken prestigereichen Gruppen (wie beispielsweise am Gymnasium) könnten sich demnach selbstbezogene Einschätzungen und Einstellungen bei Schülern günstiger entwickeln als in leistungsschwachen und weniger prestigereichen Lerngruppen.

Insgesamt ist die Anzahl der Untersuchungen zum BIRGE jedoch weniger umfangreich als zum BFLPE. Auch ist die Anzahl der Studien, die beide Bezugsgruppeneffekte gemeinsam untersucht haben, gering. In der Studie von Marsh et al. (2000), in welcher sowohl der Kontrast- als auch der Assimilationseffekt bei Schülern der high school in Hong Kong untersucht wurde, hat sich der BFLPE gegenüber dem BIRGE als dominierender Effekt herausgestellt. In einer deutschen Studie von Trautwein, Köller, Lüdtke und Baumert (2005) wurden ebenfalls beide Bezugsgruppeneffekte gemeinsam untersucht. Dazu wurde das Selbstkonzept von Schülern in mathematischen Leistungskursen der gymnasialen Oberstufe betrachtet. Anders als bei Marsh et al. (2000) dominierte in dieser Untersuchung nicht der negative BFLPE sondern der positive BIRGE. Der Assimilationseffekt wirkte sich demnach positiv auf das Selbstkonzept der Schüler im Leistungskurs Mathematik aus. In einer aktuelleren Studie von Preckel und Brüll (2010), in welcher das Fähigkeitsselbstkonzept von Schülern der fünften Klassenstufe untersucht wurde, konnte sowohl der BFLPE als auch der BIRGE in 
ähnlicher Ausprägung nachgewiesen werden. Keiner der beiden Effekte dominierte demnach das Selbstkonzept der Schüler.

Trautwein, Lüdtke, Marsh und Nagy (2009) untersuchten ebenfalls sowohl den BFLPE als auch den BIRGE. Das Ergebnis ihrer drei Studien zum mathematischen Selbstkonzept von Schülern der Sekundarstufe II war, dass in allen drei Studien der BFLPE und der BIRGE auf Individualebene belegt werden konnte. Der Assimilationseffekt ließ sich zudem in zwei der drei Studien auf Klassenebene nachweisen.

\subsubsection{Forschungsdesiderat hinsichtlich Bezugsgruppeneffekten bei Zielorientierungen}

Die vorgestellten Untersuchungen zu Bezugsgruppeneffekten haben sich ausschließlich auf Selbstkonzept, Selbstwert, intrinsische Motivation, Interesse oder Bildungsaspirationen bezogen. Bislang liegen keine Studien zu anderen Variablen vor. Aus diesem Grund wird von Seiten einiger Forscher seit geraumer Zeit eine Ausweitung der Untersuchungen zu Bezugsgruppeneffekten auf weitere Variablen gefordert (Trautwein \& Lüdtke, 2005). Da soziale Vergleichsprozesse eine zentrale Rolle bei der Entstehung von Kontrast- bzw. Assimilationseffekten spielen, sollte sich eine Ausweitung der Untersuchungen auf eine Variable beziehen, bei welcher diese Vergleichsprozesse ebenfalls eine Rolle spielen. Zielorientierungen scheinen dazu besonders geeignet zu sein, da bei diesen - insbesondere bei Leistungszielen - den sozialen Vergleichen eine wesentliche Bedeutung zukommt. Des Weiteren stellen Zielorientierungen einen zentralen Bestandteil der Motivation dar, wie es in Kapitel 2.2.2 bereits erläutert wurde. Der Effekt der Bezugsgruppe auf die Motivation konnte in zahlreichen Untersuchungen belegt werden, demnach könnte vermutet werden, dass sich dieser Effekt auch bei Zielorientierungen zeigt.

Bislang sind keine Studien bekannt, die Bezugsgruppeneffekte bei Zielorientierungen untersucht haben. Auch diesem Forschungsdesiderat soll in der vorliegenden Arbeit nachgegangen werden. Vor dem Hintergrund des Übergangs von der Grundschule auf die weiterführende Schule, welcher mit einem Wechsel der Bezugsgruppe einhergeht, erscheint die Untersuchung der Effekte, die dieser Bezugsgruppenwechsel auf verschiedene Variablen haben kann auch für Zielorientierungen sinnvoll. Da Zielorientierungen als habituelle Merkmale mit kontextueller Sensitivität angesehen werden, können diese demnach je nach Kontext variieren und sich über die Zeit verändern. Inwieweit die Lernumwelt in Form der verschiedenen Schulformen einen Einfluss auf die Veränderung der Zielorientierungen und den Zusammen- 
hang derer zu schulischen Leistungen hat, soll in der vorliegenden Dissertation u.a. untersucht werden. 


\section{$3 \quad$ Fragestellungen}

\subsection{Ableitung der Fragestellungen}

Zielorientierungen stellen ein im pädagogisch-psychologischen Kontext weit erforschtes Konstrukt dar. Sie gelten als eine wichtige Leistungsdeterminante und stehen bedeutsam im Zusammenhang mit verschiedenen motivationalen, emotionalen und kognitiven Merkmalen. Als besonders förderlich in Hinblick auf schulisches Lernen scheinen dabei Lernziele zu sein. Im Gegensatz dazu werden Vermeidungsleistungsziele als eher maladaptiv angesehen. So konnte in zahlreichen Untersuchungen ein negativer Zusammenhang zu intrinsischer Motivation, Selbstkonzept, Selbstwirksamkeitserwartungen, bzw. ein positiver Zusammenhang zu negativen Emotionen wie beispielsweise Ängstlichkeit belegt werden. Die Befundlage zu Annäherungsleistungszielen stellt sich hingegen weniger eindeutig dar. Die Auswirkungen dieser Form der Zielorientierung variieren je nach eingesetztem Instrument und untersuchter Altersgruppe. Die Forschungsergebnisse erwecken den Eindruck, dass Annäherungsleistungsziele mit zunehmendem Alter der Schüler bzw. Studierenden adaptiver und somit leistungsförderlicher werden. Die Lernumwelt scheint in diesem Kontext eine wichtige Rolle zu spielen. Doch wie sieht es bei jüngeren Schülern aus? Insbesondere im deutschsprachigen Raum mangelt es an aussagekräftigen Studien mit repräsentativen Stichproben zu diesem Thema. Aus diesem Grund wird in der vorliegenden Dissertation in Studie 1 zuerst auf grundlegende das Instrument zur Erfassung der Zielorientierungen betreffende - Aspekte eingegangen.

1. Im Zentrum der ersten Studie soll dabei die Frage stehen, inwieweit sich Zielorientierungen bereits in der Grundschule reliabel und valide messen lassen

2. und in welchem Zusammenhang diese mit schulischen Leistungen am Ende der Grundschulzeit in Klassenstufe vier stehen.

Da diese Fragestellung an Schülern in Klassenstufe vier mit einer repräsentativen Stichprobe und mit dem eingesetzten Instrument noch nicht in ausreichender Weise in der Bundesrepublik untersucht wurde, soll diesem Forschungsdesiderat in Studie 1 nachgegangen werden (Kapitel 4). Studie 1 stellt somit die Grundlage für die weiteren, auf den Ergebnissen aufbauenden, Analysen dar. 
3. Zudem wird der Frage nachgegangen, ob sich die Zusammenhänge zwischen Zielorientierungen und schulischen Leistungen unterscheiden, wenn curricular valide Schulleistungstest bzw. Ziffernoten verwendet werden.

Erwartet wird, dass Zielorientierungen höher mit Noten zusammenhängen, da in die Notenvergabe über die objektiv gemessene Schulleistung hinaus andere Aspekte, wie beispielsweise Motivation (wozu auch Zielorientierungen zu zählen sind), Arbeits- und Lernverhalten, einfließen (Helmke, 2009; Köller, 1998).

Um auszuschließen, dass es sich in Studie 1 um für das eingesetzte Instrument zur Erfassung der Zielorientierungen spezifische Ergebnisse handelt, wird eine Replikation der Befunde an einer anderen Stichprobe und mit einem anderen Instrument angestrebt (Studie 2). Dazu soll auch hier das Zusammenhangsmuster zwischen Zielorientierungen und schulischen Leistungen in Form von Schulnoten und standardisierten Testleistungen analysiert werden.

Auf der Grundlage der Ergebnisse aus Studie 1 wird dann die Entwicklung der Zielorientierungen am Übergang von der Grundschule (Klassenstufe vier) auf die weiterführende Schule (bis Klassenstufe sechs) in Studie 3 untersucht (Kapitel 5). Der Grundschulübergang stellt für viele Schüler ein kritisches Lebensereignis dar, welches mit zahlreichen Veränderungen verbunden ist. So ändern sich die Mitschüler und die Lehrkräfte, der Schulalltag und nicht zuletzt die Anforderungen, die an die Schüler gestellt werden. Der Übergang wird demnach als eine Zeit des Umbruchs und der Veränderungen angesehen, welcher nicht selten mit einer Abnahme der Lernmotivation auf Seiten der Schüler einhergeht. Besonders im angloamerikanischen Raum ist die Anzahl der Studien zum Grundschulübergang groß. Da sich das Schulsystem in der Bundesrepublik allerdings in vielen Punkten von dem US-amerikanischen unterscheidet und keine aussagekräftigen Untersuchungen zur Entwicklung von Zielorientierungen am Grundschulübergang im deutschsprachigen Raum vorliegen, soll diese Forschungslücke in der vorliegenden Arbeit geschlossen werden.

4. Die zentrale Frage ist dabei, wie sich Zielorientierungen von Klassenstufe vier bis sechs entwickeln.

5. Da sich die Lernumwelten der einzelnen Schulformen in zahlreichen Punkten voneinander unterscheiden, soll auch geprüft werden, ob es schulformspezifische Unterschiede in der Entwicklung von Zielorientierungen gibt. 
6. Eine weitere Fragestellung in Studie 3 bezieht sich auf den Zusammenhang zwischen Zielorientierungen und Schulleistungen in Form von Schulnoten. Dabei ist zum einen von Interesse, ob sich das Zusammenhangsmuster am Übergang zur weiterführenden Schule verändert und zum anderen ob sich schulformspezifische Unterschiede zeigen.

$\mathrm{Da}$ in einigen Studien postuliert wurde, dass sich das Zusammenhangsmuster zwischen Zielorientierungen und schulischen Leistungen im Laufe der Schulzeit verändert und (Annäherungs-)Leistungsziele im Gegensatz zu Lernzielen adaptiver werden, wird vermutet, dass Lernziele im Gegensatz zu Leistungszielen insbesondere an der Grundschule eine leistungsförderliche Wirkung haben. Nach dem Grundschulübergang könnten Lernziele jedoch weniger bedeutsam für schulischen Erfolg werden. Stattdessen könnten Annäherungsleistungsziele adaptiver werden und schulische Leistungen positiv vorhersagen. Auch in Studie 3 soll der Einfluss der Schulform auf den Zusammenhang zwischen Zielorientierungen und schulischen Leistungen untersucht werden. Da die Lernumwelt am Gymnasium als herausfordernder und kognitiv aktivierender beschrieben werden kann, wird vermutet, dass sich die potenziell positive Wirkung von Lernzielen in dieser Lernumwelt besonders zeigt.

Mit dem Grundschulübergang ändern sich nicht nur räumliche und anforderungsbezogene Merkmale, es findet auch ein Bezugsgruppenwechsel statt, welcher in der Literatur insbesondere in Bezug auf das Fähigkeitsselbstkonzept, Selbstwert, intrinsische Motivation und Interesse untersucht wurde. So kann zum einen ein negativer Zusammenhang zwischen der mittleren Leistungsstärke der Bezugsgruppe und den verschiedenen Variablen bestehen (BigFish-Little-Pond-Effekt), zum anderen kann sich aber auch das hohe Prestige einer leistungsstarken Bezugsgruppe positiv auf u.a. das Selbstkonzept der Schüler auswirken (Basking-InReflected-Glory-Effekt). Da diese Kontrast- bzw. Assimilationseffekte auf der Basis sozialer Vergleichsprozesse entstehen und auch bei Zielorientierungen soziale Vergleiche eine wichtige Rolle spielen und Zielorientierungen zudem einen bedeutsamen Bestandteil der Motivation darstellen, soll in Studie 4 (Kapitel 6) die Forschung zu Bezugsgruppeneffekten auf Zielorientierungen ausgeweitert werden.

7. Im Zentrum von Studie 4 soll dabei die Frage stehen, inwieweit sich Bezugsgruppeneffekte nach dem Grundschulübergang auch bei Zielorientierungen unter Kontrolle der schulischen Leistungen in Form von Schulnoten und curricular validen Testleistungen finden lassen. 
Es werden Unterschiede bei den jeweiligen Zielorientierungen erwartet. So wird vermutet, dass die Entwicklung der Lernziele negativ am Gymnasium verläuft und sich somit der BFLPE auf die Lernziele dieser Schüler auswirkt.

8. Eine weitere zentrale Frage in Studie 4 ist, ob sich die Bezugsgruppeneffekte durch Kontrolle der Schulnoten nach dem Übergang in Klassenstufe fünf erklären lassen.

Da die mittlere Leistungsstärke der Gruppe den BFLPE verursacht, wird vermutet, dass sich dieser bei vergleichbarer Leistungsstärke der Schüler verringert bzw. verschwindet.

\subsection{Aufbau der Untersuchung}

Um die abgeleiteten Fragestellungen im befriedigenden Maße beantworten zu können, bedarf es eines längsschnittlichen Untersuchungsdesigns, welches nicht erst nach dem Grundschulübergang einsetzt, sondern bereits in der Grundschule bevor die Schüler auf die weiterführende Schule wechseln. Dieser Bedarf ergibt sich daraus, weil zum einen in Studie 1 die Konstruktvalidität von Zielorientierungen und der Zusammenhang derer zu schulischen Leistungen am Ende der Grundschulzeit untersucht und zum anderen weil die Entwicklung von Zielorientierungen am Übergang zu den verschiedenen Schulformen des deutschen Schulsystems analysiert werden soll. Um den potenziellen Einfluss der Schulformen auf die Entwicklung von Zielorientierungen und deren Zusammenhang mit schulischen Leistungen zu untersuchen, ist es notwendig die Schüler bereits vor dem Grundschulübergang zu befragen, um auszuschließen, dass mögliche Unterschiede bereits vor dem Schulwechsel bestanden und nicht auf die jeweilige Schulform bzw. Bezugsgruppe zurückzuführen sind.

Die Übergangsstudie, welche eine Erweiterung der Trends in International Mathematics and Science Study (TIMSS) 2007 darstellt und in Kooperation zwischen dem Max-Planck-Institut für Bildungsforschung (MPIB Berlin), dem Arbeitsbereich Empirische Bildungsforschung der Freien Universität Berlin, der Universität Potsdam, dem Institut für Schulentwicklungsforschung (Technische Universität Dortmund) und dem Institut zur Qualitätsentwicklung im Bildungswesen (Humboldt-Universität zu Berlin) entstanden ist, stellt eine Längsschnittstudie beginnend in Klassenstufe vier dar (für weiterführende Informationen siehe Maaz et al., 2010). Die Teilnahme Deutschlands an der TIMS-Studie 2007 bot eine ideale Gelegenheit mit einem geringen Zusatzaufwand eine Reihe von - den Grundschulübergang 
betreffende - Merkmalen zu erfassen. So wurden u.a. schülerbezogene Persönlichkeitsmerkmale wie beispielsweise intrinsische Motivation, Selbstkonzept, Leistungsangst, aber auch Zielorientierungen vor sowie auch nach dem Grundschulübergang erhoben. Des Weiteren wurden zusätzlich zu den TIMSS-Testleistungen die Schulnoten der Schüler erfasst.

Die Untersuchungspopulation entsprach weitestgehend der der TIMS-Studie: alle Schüler, die im Schuljahr 2006/07 eine vierte Klasse auf einer Regelschule besuchten und in einem Bundesland wohnten, in welchem der Grundschulübergang nach der vierten Klassenstufe stattfand $^{8}$, waren Teil der Grundgesamtheit (siehe Becker et al., 2010 für weiterführende Angaben zur Stichprobenziehung). Der Zeitraum der Befragungen der Schüler der TIMSSÜbergangsstudie erstreckte sich von April/Mai 2007 bis September/Oktober 2008 (insgesamt sechs Erhebungen, wobei die Schüler lediglich zu vier Zeitpunkten befragt wurden). Die TIMSS-Testdurchführung fand am Ende der vierten Klassenstufe an zwei Testtagen (April/Mail 2007) statt. Am ersten Testtag wurden die Mathematik- und Naturwissenschaftstests und der erste Teil der Schülerfragebögen (internationaler TIMSS-Fragebogen und TIMSSÜbergangsstudie-Fragebogen) ausgefüllt. Am zweiten Testtag wurden die Aufgaben des IQB zu den nationalen Bildungsstandards im Fach Deutsch bearbeitet sowie ein kognitiver Grundfähigkeitstest und die nationale Ergänzung des TIMSS-Schülerfragebogens eingesetzt.

Die Analysen der vorliegenden Arbeit basieren auf insgesamt drei Messzeitpunkten. Der erste Messzeitpunkt lag am Ende der vierten Klassenstufe und die beiden anderen in der Mitte der Klassenstufe fünf bzw. zu Beginn der Klassenstufe sechs. Insgesamt nahmen an der Untersuchung $N=5242$ Schülerinnen (49,4\%) und Schüler aus 253 Schulen teil (eine Klasse pro Schule), wobei 13,9 Prozent der Schüler einen Migrationshintergrund aufwiesen (ein Elternteil oder beide Elternteile nicht in Deutschland geboren). Das Alter der untersuchten Schüler lag zum ersten Messzeitpunkt bei $M=10.42$ Jahren $(S D=0.50)$.

Wie auch in anderen Längsschnittstudien kam es in der TIMSS-Übergangsstudie zu Datenausfällen. Von den insgesamt 5242 Schülern gaben 2212 der Eltern (42.2 \%) ihre Adresse an und konnten somit für die postalischen Nachbefragungen kontaktiert werden. An den Befragungen nach dem Grundschulübergang nahmen circa 75 Prozent der Haushalte in der Mitte der Klassenstufe fünf und etwa 65 Prozent zu Beginn der sechsten Klassenstufe teil. Somit lag die Beteiligung an den Befragungen nach dem Übergang bei circa 80 Prozent der

\footnotetext{
${ }^{8}$ Insgesamt nahmen somit 13 Bundesländer an der Untersuchung teil. Berlin, Brandenburg und MecklenburgVorpommern wurden ausgeschlossen, da in diesen Bundesländern der Grundschulübergang in der Regel erst nach der sechsten Klassenstufe stattfindet.
} 
Haushalte, von denen Angaben zur Adresse vorlagen (für weiterführende Angaben siehe Becker et al., 2010, S. 115ff.).

Für die längsschnittlichen Analysen (Studie 3 und 4) wurden die Daten von $N=1646$ Schülerinnen $(49,4 \%)$ und Schülern ausgewertet, wobei lediglich Schüler berücksichtigt wurden, welche an der Erhebung in Klassenstufe vier und an mindestens einer der beiden anderen Erhebungen in Klassenstufe fünf oder sechs teilnahmen. Die Verteilung der Schüler auf die verschiedenen Schulformen gestaltete sich wie folgt: Gymnasium: $n=928$, Realschule: $n$ =436, Hauptschule: $n=158$, andere Schulformen: $n=124)$. Da die Stichprobengrößen der Real-, Hauptschule und den anderen Schulformen relativ klein waren, wurden diese Schulformen zusammengefasst $(n=718)$.

Um die Repräsentativität der Stichprobe einzuschätzen, wurden Selektivitätsanalysen durchgeführt (für weiterführende Informationen siehe Becker et al., 2010, S. 118f.). Die Ergebnisse der Analysen belegen, dass die Längsschnittstichprobe (Teilnahme Klassenstufe fünf und sechs) eine positive Auswahl darstellt. So liegt eine Überrepräsentierung von Schülern vor, welche eine Gymnasialempfehlung am Ende der Grundschule hatten. Zudem verfügten die Schüler der Längsschnittstichprobe in Klassenstufe 4 über bessere Schulnoten in Deutsch, Mathematik und Sachunterricht sowie über bessere Testleistungen in Deutsch, Mathematik und Naturwissenschaften (etwa eine halbe Standardabweichung). Ebenso war die sozioökonomische Stellung der Eltern in der Längsschnittstichprobe etwa eine halbe Standardabweichung höher.

Zweifaktorielle Varianzanalysen mit den Faktoren Übergangsempfehlung und Zugehörigkeit zur Längsschnittstichprobe und den Kriterien Noten, Testleistungen und sozioökonomische Stellung der Eltern erbrachten keinen signifikanten Interaktionseffekt (Laufbahnempfehlung $\times$ Zugehörigkeit zur Längsschnittstichprobe). Somit liegt zwar ein systematischer Dropout vor, jedoch unterscheiden sich die Schülergruppen mit unterschiedlichen Übergangsempfehlungen nicht systematisch voneinander. Zieht man die Schulformentscheidung der Eltern anstelle der Übergangsempfehlungen am Ende von Klasse vier heran, ergeben sich ähnliche Ergebnisse. Im Hinblick auf die Schulleistung und die soziale Herkunft sprechen die Befunde für eine in etwa vergleichbare Panelmortalität zwischen den Schulformen, wenn man von einer hohen Übereinstimmung zwischen Schulformentscheidung und deren Realisierung ausgeht (siehe Becker et al., 2010, S. 118f.).

Im Schülerfragebogen der TIMSS-Übergangsstudie wurde eine Reihe von Schülermerkmalen erfasst. Im Folgenden werden die für die vorliegende Arbeit relevanten Merkmale 
erläutert. Dabei handelt es sich um Zielorientierungen, intrinsische Motivation, akademisches Selbstkonzept und Leistungsangst.

Die Zielorientierungen wurden mit ursprünglich elf Items erhoben. Dabei handelte es sich bei den Items der (Annäherungs-)Lernziele um vier Items, welche aus den Patterns of Adaptive Learning Scales (PALS; revised version; Midgley et al., 2000) stammen und ins Deutsche übersetzt wurden. Die vier Items der Annäherungsleistungsziele basierten auf Items aus der deutschen Fassung der Motivational Orientation Scales von Köller und Baumert (1998) (Original von Nicholls, Patashnick \& Nolen, 1985). Die drei Items der Vermeidungsleistungsziele beruhten auf einer Eigenentwicklung von Schwinger und Wild (2006). Aufgrund komplexer Faktorladungen eines Items in einer explorativen Faktorenanalyse wurde dieses jedoch aus den weiteren Analysen ausgeschlossen. Die jeweiligen Items der Zielorientierungen wurden bereichsübergreifend formuliert (nicht auf die einzelnen Schulfächer bezogen) und waren auf einer vierstufigen Likert-Skala $(1=$ stimmt genau bis $4=$ stimmt gar nicht) einzuschätzen (Itemübersicht siehe Anhang A). In der Originalversion des PALS wurde ein fünfstufigen Antwortformat gewählt, jedoch kann es bei diesem Antwortformat - aufgrund der neutralen Mittelkategorie - zu einer Verzerrung der Interpretation der Befunde kommen (z.B. Moosbrugger \& Kelava, 2008, S. 53f.). Aus diesem Grund wurde sich für ein vierstufiges Antwortformat entschieden.

Die Erfassung der intrinsischen Motivation der Schüler erfolgte mittels acht Items, von denen vier epistemische Neugier (Berlyne, 1978; Litman \& Spielberger, 2003) und vier Lernfreude maßen (Itemübersicht siehe Anhang B). Da sich die beiden Dimensionen empirisch nicht trennen ließen, wurde in der postalischen Befragung eine gekürzte Version der Skala eingesetzt. Ein Beispielitem lautet: „Ich bin immer ganz neugierig, wenn ich Neues lernen kann“. Die Items wurden eigens für die TIMSS-Übergangsstudie entwickelt und pilotiert. Das Antwortformat war vierstufig ( 1 = stimmt gar nicht bis $4=$ stimmt genau). Die internen Konsistenzen lagen bei $\alpha=.81 \mathrm{zu} \mathrm{T} 1$, bei $\alpha=.89$ zu T2 und bei $\alpha=.80 \mathrm{zu}$ T3.

Zur Erfassung des akademischen Selbstkonzepts der Schüler wurden drei Items (z.B.: „Ich bin in den meisten Schulfächern gut.“) verwendet, welche eine Eigenentwicklung der TIMSS-Übergangsstudie in Anlehnung an den Self Description Questionnaire (SDQ, Marsh, 1990) darstellen. Die Items kamen bereits bei PISA 2000 zum Einsatz (vgl. Kunter et al., 2002). Das Antwortformat war vierstufig ( $1=$ stimmt gar nicht bis $4=$ stimmt genau) und die internen Konsistenzen lagen bei $\alpha=.81 \mathrm{zu} \mathrm{T} 1$, bei $\alpha=.82 \mathrm{zu}$ T2 und bei $\alpha=.83 \mathrm{zu}$ T3.

Die Leistungsangst der Schüler wurde mittels neun Items erfasst, mit welchen nach der Gefühlslage während der letzten Klassenarbeit gefragt wurde. Dabei bezogen sich vier 
Items auf worry-Kognitionen (Eigenentwicklung vgl. Helmke, 1992; z.B.: „Ich dachte daran, was ich alles nicht kann.“) und fünf Items auf die Wahrnehmung physiologischer Erregung (emotionality) (deutsche Fassung des Test Anxiety Inventory, vgl. Hodapp, Laux \& Spielberger, 1982; z.B.: „Das Herz schlug mir bis zum Hals.“). Das Antwortformat war vierstufig (1 = stimmt gar nicht bis $4=$ stimmt genau). Die interne Konsistenz lag zu T1 bei $\alpha=.91$.

Als Indikator für die Schulleistung der Schüler wurden die TIMSS-Testergebnisse aus den Bereichen Naturwissenschaften und Mathematik herangezogen. Der Leistungstest Naturwissenschaften bestand aus insgesamt 174 Testaufgaben (93 im Multiple-Choice-Format, 81 in einem kurzen offenen Antwortformat, $\alpha=.80$ ). Dabei bezogen sich die Aufgaben inhaltlich auf die Bereiche Biologie (43\%), Physik (37\%) und Chemie (21\%). Zudem beinhaltete der Test Aufgaben zum Reproduzieren (4\%), Anwenden (26\%) und Problemlösen (20\%) (vgl. Bonsen, Lintorf, Bos \& Frey, 2008).

Der Mathematiktest setzte sich aus insgesamt 179 Aufgaben zusammen (96 MultipleChoice-Items und 83 Aufgaben im offenem Antwortformat, $\alpha=.83$ ). Inhaltlich teilten sich die Aufgaben wie folgt auf: Arithmetik (52\%), Geometrie/Messen (34 \%) und Daten (15\%). Zudem enthielt der Test Items der kognitiven Anforderungsbereiche Reproduzieren (39 \%), Anwenden (39\%) und Problemlösen (22 \%) (vgl. Bonsen et al., 2008).

Die Ergebnisse der Tests Naturwissenschaften und Mathematik wurden raschskaliert und auf eine Metrik von $M=150$ und $S D=10$ standardisiert. Die Aufgaben verteilten sich auf 14 Blöcke mit jeweils 10 bis 15 Aufgaben (Multi-Matrix-Design), wobei jeder Schüler ein Testheft mit je zwei Mathematik- und zwei Sachunterrichtsblöcken bearbeitete (ca. 20 Minuten pro Block).

Die Deutschleistungen wurden vom Institut zur Qualitätsentwicklung im Bildungswesen im Zuge der Graduierung der Bildungsstandards für Grundschulen erfasst. Dazu wurden 446 Items $(\alpha=.81)$ entwickelt, welche sich auf die von der Kultusministerkonferenz 2004 verabschiedeten Bildungsstandards für das Fach Deutsch in der Primarstufe (Jahrgangsstufe vier) bezogen. Die Items wurden pilotiert und parallel zu TIMSS normiert (Metrik von $M=$ 150 und $S D=10$ ). Theoretisch lassen sich die eingesetzten Items auf die vier Kompetenzbereiche Lesen, Hören, Sprachgebrauch und Rechtschreibung zuordnen. Jeder Schüler bearbeitete Aufgaben aus zwei bis drei Kompetenzbereichen (Bearbeitungsdauer: ca. 40 Minuten) (siehe Becker et al., 2010, S. 112).

Neben den TIMSS-Testleistungen aus den Bereichen Naturwissenschaften, Mathematik und Deutsch wurden die Schulnoten zu allen drei Messzeitpunkten in den Fächern Deutsch, Mathematik und erste Fremdsprache bzw. zusätzlich im Fach Sachunterricht in 
Klassenstufe vier erhoben. Die Notenangaben stammen von der jeweiligen Schule und wurden direkt aus der Schülerteilnahmeliste übernommen.

Zudem wurde die kognitive Grundfähigkeit der Schüler erfasst, da diese allgemein als relevanter Prädiktor von Schulleistungen gilt. Dazu wurde der Subtest N2 des Kognitiven Fähigkeits-Tests für vierte Klassen (KFT 4 R; Heller \& Perleth, 2000) in zwei parallelen Versionen eingesetzt (Version A: $\alpha=.92$, Version B: $\alpha=.93$ ). Die Aufgabe bestand darin, Analogien zwischen zwei Figurenpaaren zu bilden.

Wie in den meisten Untersuchungen, wies auch der verwendete Datensatz fehlende Werte auf. Dabei lag der Anteil fehlender Werte bei den untersuchten Variablen des Schülerfragebogens zu T1 bei 17 Prozent und bei den Testleistungen in Mathematik, Naturwissenschaft und den kognitiven Grundfähigkeiten bei unter fünf Prozent. Bei den Deutschleistungen lag der Anteil fehlender Werte bei 35,8 Prozent, da diese nur bei zwei Dritteln der Stichprobe erfasst wurden. Die fehlenden Werte wurden mit Hilfe multipler Imputationen (vgl. Graham, Cumsille \& Elek-Fisk, 2003) unter Einbeziehung weiterer Hintergrundmerkmale (z.B. Geschlecht, Bundesland, Laufbahnempfehlung, soziale Herkunft) geschätzt. Anders als bei fallweisem oder paarweisem Ausschluss von Personen mit fehlenden Werten führt das Verfahren der Multiple Imputation zu erwartungstreueren und effizienteren Parameterschätzungen (Little \& Rubin, 2002). Um die Prüfung der faktoriellen Validität mit allen Schülern durchführen zu können, wurden fehlende Werte der Zielorientierungen auf Itemebene ersetzt, bei allen anderen Skalen auf Skalenebene. In Studie 1 kam das Programm Amelia II (Version:1.2-13, Honaker, King \& Blackwell, 2006) zum Einsatz, mit welchem fünf vollständige Datensätze erzeugt wurden. Auf Grundlage der Berechnungsvorschrift von Rubin (1987) wurden die fünf Datensätze von dem Statistikprogramm Mplus 6.1 (Muthén \& Muthén, 19982010) zu einer Gesamtschätzung kombiniert. In Studien 3 und 4 erfolgten die Multiplen Imputationen mit Mplus 6.1 bzw. Mplus 6.11 (Muthén \& Muthén, 1998-2011). Auch in diesen Fällen wurden fünf vollständige Datensätze erstellt, welche bei den Analysen von Mplus zu einer Gesamtschätzung kombiniert wurden. 


\title{
4 Zielorientierungen und schulische Leistungen am Grundschulüber- gang (Studien 1 und 2)
}

\subsection{Zusammenfassung und Abstract}

Zusammenfassung. Die vorliegende Arbeit beschäftigte sich mit der Frage, in welchem Zusammenhang Zielorientierungen und Schulleistungen von Schülern am Ende der vierten bzw. zu Beginn der fünften Jahrgangsstufe stehen. Die Daten von zwei Studien (Studie 1: $N=5$ 242; Studie $2 N:=294$ ) wurden analysiert, in denen Zielorientierungen mit zwei unterschiedlichen Instrumenten und Schulleistungen über Noten hinaus mit curricular validen Leistungstests gemessen wurden. Während die Lernziel- und die Vermeidungsleistungsziele in keinem bzw. nur sehr schwachem Zusammenhang mit den Schulleistungen standen, hingen die Annäherungsleistungsziele in beiden Studien negativ mit den Schulleistungen zusammen. Die Befunde der Testleistungen und Noten unterschieden sich nur gering. Zusammenfassend deuten die Befunde darauf hin, dass in dieser Altersgruppe nicht - wie erwartet - die Vermeidungs-, sondern die Annäherungsleistungsziele Ausdruck eines weniger günstigen lernbezogenen Erlebens- und Verhaltensmusters sind.

\begin{abstract}
The present article focused on the relation between students' achievement goals and school achievement at the end of grade four and the beginning of grade five. Data of two studies (study 1: $N=5,242$; study $2 N$ : $=294$ ) were analyzed, in which we measured achievement goals with two different instruments and school achievement with standardized achievement tests in addition to classroom grades. While mastery and performance-avoidance goals showed no or just a small relation to school achievement, were performance-approach goals negatively related to school achievement in both studies. The results for test scores and class grades differed only slightly. In conclusion, these results indicate that, contrary to our expectations, not performance-avoidance goals but performance-approach goals were an expression of a less favorable learning oriented behavior pattern within this age group.
\end{abstract}

\footnotetext{
${ }^{9}$ Dieser Abschnitt basiert auf einem bei der Zeitschrift Unterrichtswissenschaft eingereichten Manuskript (Paulick, Watermann \& Nückles, 2011c).
} 


\subsection{Einleitung}

Die Annahme, dass Zielorientierungen eine bedeutsame Rolle beim Lernen spielen, wird vielfach in der Literatur vertreten (vgl. Schunk et al., 2008, Kap. 5). Unter anderem wird die Bedeutung von Zielorientierungen für Emotionen (Pekrun et al., 2006) sowie für den Einsatz bestimmter Lernstrategien (Bong, 2009; Elliot et al., 1999) als Begründung angeführt. Der Zusammenhang zwischen Zielorientierungen und Schulleistungen wurde in der Primarstufe jedoch bislang kaum untersucht. Vor allem fehlt es an Studien, in denen Schulleistungen mit curricular validen Tests gemessen werden. Ansetzend an den vielfach in der Literatur berichteten Zusammenhängen zwischen Zielorientierungen und Schulleistungen sollen in der vorliegenden Untersuchung, unter Verwendung curricular valider Schulleistungstests, die Zusammenhänge der Variablen am Grundschulübergang geprüft werden. Die Zielorientierungen wurden mit einem national (Skalen zur Erfassung der Lern- und Leistungsmotivation; SELLMO, Spinath et al., 2002) und einem international (Patterns of Adaptive Learning Scales; PALS, Midgley et al., 2000) etablierten Instrument erfasst.

\subsection{Zielorientierung}

Zielorientierungen stellen die motivationale Ausrichtung von Personen in Lern- und Leistungskontexten dar und gelten als eine wichtige Determinante des Leistungshandelns (z.B. Harackiewicz, Barron, Tauer \& Elliot, 2002). Es lassen sich zwei grundlegende Dimensionen von Zielorientierungen unterscheiden: Lern- und Leistungszielorientierungen.

Bei einer Lernzielorientierung strebt die Person primär eine Steigerung der eigenen Kompetenzen an, bei einer Leistungszielorientierung steht hingegen das Demonstrieren der eigenen Leistung vor anderen im Vordergrund. Es wird zusätzlich die Unterteilung in Annäherungs- und Vermeidungsziele (Elliot, 1997) vorgenommen. Unter Annäherungsleistungszielen wird das Streben nach Demonstration der eigenen Fähigkeiten vor anderen verstanden, wohingegen Vermeidungsleistungsziele das Verbergen fehlenden Könnens und Wissens beinhalten.

Zur Erfassung der Zielorientierungen existiert eine Reihe von Instrumenten. Neben den bereits erwähnten SELLMO und PALS lassen sich unter anderem die Skalen zur Erfassung der Zielorientierungen von Köller und Baumert (1998), die Motivational Orientation Scales (MOS) von Nicholls (1989) und der Achievement Goal Questionnaire (AGQ) von Elliot und McGregor (2001) nennen. Mit diesen Instrumenten lassen sich Zielorientierungen 
reliabel messen, lediglich in Bezug auf die Konstrukt- und Kriteriumsvalidität konnte für die im anglo-amerikanischen Raum eingesetzten Instrumente festgestellt werden, dass Unterschiede in der Operationalisierung zu Unterschieden in den Zusammenhängen etwa mit Motivation und Schulleistungen führen (vgl. Hulleman et al., 2010).

Nur wenige dieser Instrumente wurden für den Einsatz an Grundschülern entwickelt. Lediglich die SELLMO und mit Einschränkungen die PALS gelten als valide und reliable Instrumente zur Erhebung von Zielorientierungen bei Schülern der vierten bzw. fünften Klassenstufe.

\subsection{Zielorientierung und schulische Leistungen}

Der Einfluss der Zielorientierung auf Leistungen konnte in zahlreichen Untersuchungen nachgewiesen werden (siehe Metanalyse von Hulleman et al., 2010). Insbesondere zeigten sich Lernziele als leistungsfördernd. Vermeidungsleistungsziele konnten hingegen oftmals mit niedrigeren Leistungen in Zusammenhang gebracht werden. Bei Annäherungsleistungszielen sind die Befunde weniger konsistent. Midgley, Kaplan und Middleton (2001) sind nach Sichtung zahlreicher Untersuchungen $\mathrm{zu}$ dem Ergebnis gekommen, dass unter bestimmten Umständen (z.B. in kompetitiven Lernumwelten) und für bestimmte Personen (z.B. Jungen, ältere Schüler) positive Auswirkungen der Annäherungsleistungsziele auf schulische Leistungen gefunden werden konnten. In der Literatur wird der Zusammenhang zwischen Zielorientierungen und Schulleistungen vor allem mit dem Einsatz bestimmter Lernstrategien begründet (z.B. Köller, 1998). So wird angenommen, dass eine hohe Lernzielorientierung mit der aktiven Nutzung von Tiefenverarbeitungsstrategien einhergeht, wohingegen eine hohe Leistungszielorientierung (nicht differenziert nach Annäherungs- und Vermeidungsleistungsziele) verstärkt mit der Anwendung von Oberflächenstrategien verbunden ist (u.a. Bouffard et al., 1995). Pekrun und Mitarbeiter betonen die Rolle von Zielorientierungen für Emotionen beim Lernen, wobei Lernziele mit positiven Emotionen (z.B. Freude) und Vermeidungsleistungsziele mit negativen Emotionen (z.B. Leistungsangst) in Verbindung gebracht werden (Pekrun et al., 2006).

Für den Primarstufenbereich wurde bislang kein (Anderman \& Midgley, 1997; Finsterwald, 2006) oder nur ein schwacher (z.B. Bong, 2009) Zusammenhang zu den schulischen Leistungen nachgewiesen. Im Zuge der Validierung der SELLMO konnte eine negative Korrelation zwischen Lernzielen und den Noten in der vierten Klassenstufe belegt werden. Mit einer höheren Lernzielorientierung gingen bessere Noten einher (und umgekehrt). In all 
diesen Untersuchungen wurden Ziffernnoten als Indikator der Schulleistung eingesetzt. Köller (1998) prüfte den Zusammenhang zwischen Zielorientierung und Schulleistung mit Hilfe curricular valider Tests bei Schülern der siebten Jahrgangsstufe im Längsschnitt und fand bei lernzielorientierten (aufgabenorientierten) Schülern höhere Lernraten als bei leistungszielorientierten (ichorientierten) Schülern. Es mangelt an Untersuchungen im deutschsprachigen Raum, die den Zusammenhang zwischen Zielorientierungen und Schulleistungen anhand curricular valider Leistungstests im Primarbereich untersucht haben.

\subsection{Fragestellung und Hypothesen}

Folgenden Fragestellungen wird in der vorliegenden Arbeit nachgegangen:

1. Ergibt sich beim Einsatz einer adaptierten Kurzversion der PALS an einer repräsentativen Stichprobe von Viertklässlern die theoretisch postulierte 3Faktorenstruktur (Lern-, Annäherungsleistungs-, Vermeidungsleistungsziele)?

2. In welchem Zusammenhang stehen Zielorientierungen mit selbstbezogenen Fähigkeitskognitionen, mit der Lernmotivation und Emotionen? Um diese Frage nach der Konstruktvalidität zu prüfen, wurden die Zusammenhänge zum akademischen Selbstkonzept, der intrinsischen Motivation und der Leistungsangst betrachtet. Wir erwarteten positive Zusammenhänge zwischen Lernzielen und dem akademischen Selbstkonzept sowie der intrinsischen Motivation und einen negativen Zusammenhang mit der Leistungsangst. In Hinblick auf die Vermeidungsleistungsziele hingegen erwarteten wir einen positiven Zusammenhang mit der Leistungsangst und negative Zusammenhänge mit dem Selbstkonzept und der intrinsischen Motivation. Bei den Annäherungsleistungszielen konnten keine klaren Erwartungen formuliert werden, da die Befundlage hierzu bislang inkonsistent ist.

3. Welcher Zusammenhang besteht zwischen Zielorientierungen und Schulleistungen am Grundschulübergang? Vermutet wurden ein positiver Zusammenhang bei Lernzielen und ein negativer Zusammenhang bei Vermeidungsleistungszielen. Die Hypothese zum Zusammenhang zwischen Annäherungsleistungszielen und Schulleistung konnte aufgrund der inkonsistenten Forschungsergebnisse nicht klar formuliert werden. 
4. Unterscheiden sich die Zusammenhänge zwischen Zielorientierungen und Schulleistungen, wenn man curricular valide Schulleistungstests bzw. Ziffernnoten verwendet? Wir erwarteten höhere Zusammenhänge mit den Noten, da in diese über die objektiv gemessene Schulleistung hinaus auch Aspekte der Motivation (wozu auch die Zielorientierungen zu zählen sind) sowie des Arbeits- und Lernverhaltens einfließen (Helmke, 2009; Köller, 1998).

Zur Beantwortung der Fragen wurden zwei Studien durchgeführt. In Studie 1 sind wir den oben aufgeführten Fragestellungen und Hypothesen anhand einer repräsentativen Stichprobe mit einem ins Deutsche übersetzten und modifizierten Instrument zur Erfassung von Zielorientierungen (PALS) nachgegangen. Studie 2 versuchte eine Replikation der Ergebnisse mit einem im deutschen Sprachraum bereits etablierten Instrument (SELLMO). 


\subsection{Studie 1}

\subsubsection{Methode}

Die Daten der TIMS-Studie wurden analysiert, welche in Kooperation zwischen dem Max-Planck-Institut für Bildungsforschung (MPIB Berlin), dem Arbeitsbereich Empirische Bildungsforschung der Freien Universität Berlin, dem Institut für Schulentwicklungsforschung (Technische Universität Dortmund) und dem Institut zur Qualitätsentwicklung im Bildungswesen (Humboldt-Universität zu Berlin) erhoben wurden und eine Erweiterung des nationalen Teils der Trends in International Mathematics and Science Study (TIMSS) 2007 sind (Bos et. al., 2008; Maaz et al., 2010).

Die schriftliche Befragung (Schulleistungstests und Schülerfragebogen) fand am Ende der vierten Klassenstufe (April/Mai 2007) an zwei Testtagen statt. Am ersten Testtag bearbeiteten die Schüler standardisierte Tests zur Erfassung mathematischer und naturwissenschaftlicher Kompetenzen und anschließend den internationalen Schülerfragebogen (Testzeit: ca. 150 Minuten). Am zweiten Testtag folgten Aufgaben des IQB zu den nationaler Bildungsstandards im Fach Deutsch, ein kognitiver Grundfähigkeitstest und die nationale Ergänzung des TIMSS-Schülerfragebogens (Testzeit: ca. 130 Minuten). In der nationalen Erweiterung der TIMS-Studie wurden die Zielorientierungen sowie weitere emotionale, motivationale und kognitive Merkmale der Schüler erhoben.

\subsubsection{Stichprobe}

Die Stichprobe bestand aus $N=5242$ Schülerinnen (49,4\%) und Schülern aus 253 Grundschulen (eine Klasse pro Schule) bundesweit (ausgenommen Berlin, Brandenburg, Mecklenburg-Vorpommern). Das Alter der untersuchten Schüler lag bei $M=10.42$ Jahren $(S D=0.50) .13,9$ Prozent der Schüler wiesen einen Migrationshintergrund auf (ein Elternteil oder beide Elternteile nicht in Deutschland geboren).

\subsubsection{Instrumente und Operationalisierung}

Zielorientierungen. In Studie 1 haben wir Zielorientierungen mit elf Items erhoben. Dabei wurden zur Erfassung der (Annäherungs-) Lernziele $(\alpha=.70)$ vier ins Deutsche übersetzte Items aus den PALS (revised version; Midgley et al., 2000) verwandt. Annäherungs- 
leistungsziele $(\alpha=.83)$ wurden mit vier Items gemessen, welche auf der deutschen Version der Motivational Orientation Scales (MOS; Köller \& Baumert, 1998; Original von Nicholls, et al., 1985) basierten. Vermeidungsleistungsziele $(\alpha=.59)$ wurden mit zwei Items erfasst, welche auf einer Entwicklung von Schwinger und Wild (2006) basierten. Es handelte sich um ursprünglich elf domänenübergreifend formulierte Items (nicht auf einzelne Schulfächer bezogen), welche auf einer vierstufigen Likert-Skala ( $1=$ stimmt genau bis $4=$ stimmt gar nicht) einzuschätzen waren (Itemübersicht siehe Anhang A). Die Entscheidung für ein vierstufiges anstelle des fünfstufigen Antwortformats, wie es in der Originalversion des PALS verwendet wurde, liegt in der oftmals nachgewiesenen Problematik einer neutralen Mittelkategorie begründet, die zu einer Verzerrung der Interpretation der Befunde führen kann (z.B. Moosbrugger \& Kelava, 2008, S. 53f.). Aufgrund komplexer Faktorladungen eines Items (Vermeidungsleistungsziele) in einer explorativen Faktorenanalyse wurde dieses aus den weiteren Analysen ausgeschlossen.

Schulleistungen. Als Indikator für die Schulleistung galten die TIMSS-Testergebnisse aus den Bereichen Naturwissenschaften (93 Multiple-Choice-Items und 81 Aufgaben im offenem Antwortformat, $\alpha=.80$ ) und Mathematik (96 Multiple-Choice-Items, 83 Items im Kurzantwortformat, $\alpha=.83$ ), welche raschskaliert wurden (Metrik von $M=150$ und $S D=10$ ). Die Aufgaben verteilten sich auf 14 Blöcke mit jeweils 10 bis 15 Aufgaben (Multi-MatrixDesign), wobei jeder Schüler ein Testheft mit je zwei Mathematik- und zwei Sachunterrichtsblöcken bearbeitete (ca. 20 Minuten pro Block). Die Deutschleistungen wurden vom IQB im Zuge der Graduierung der Bildungsstandards für Grundschulen erfasst. Dazu wurden auf Grundlage der von der Kultusministerkonferenz 2004 verabschiedeten Bildungsstandards für das Fach Deutsch in der Primarstufe (Jahrgangsstufe vier) 446 Items $(\alpha=.81)$ entwickelt, pilotiert und parallel zu TIMSS normiert (Metrik von $M=150$ und $S D=10$ ). Diese Items bezogen sich auf die vier Kompetenzbereiche Lesen, Hören, Sprachgebrauch und Rechtschreibung. Jeder Schüler bearbeitete Aufgaben aus zwei bis drei Kompetenzbereichen (Bearbeitungsdauer: ca. 40 Minuten).

Außerdem wurden die letzten Halbjahresnoten der Fächer Deutsch, Mathematik und Sachunterricht erhoben. Die Notenangaben stammen von der jeweiligen Schule und wurden direkt aus der Schülerteilnahmeliste übernommen.

Intrinsische Motivation. Die Skala setzte sich aus acht Items zusammen (Itemübersicht siehe Anhang B), von denen die eine Hälfte epistemische Neugier (Berlyne, 1978; Litman \& Spielberger, 2003) und die andere Hälfte Lernfreude maß. Empirisch ließen sich die beiden Dimensionen nicht trennen. Ein Beispielitem lautet: „Ich bin immer ganz neugierig, wenn ich 
Neues lernen kann“. Das Antwortformat war vierstufig $(1=$ stimmt gar nicht bis $4=$ stimmt genau). Die interne Konsistenz lag bei $\alpha=.81$.

Akademisches Selbstkonzept. Es wurden drei Items (z.B.: „Ich bin in den meisten Schulfächern gut.“; Itemübersicht siehe Anhang B) aus dem Self Description Questionnaire (SDQ, Marsh, 1990) verwendet, welche bereits bei PISA 2000 zum Einsatz kamen (vgl. Kunter et al., 2002). Das Antwortformat war vierstufig $(1=$ stimmt gar nicht bis $4=$ stimmt genau) $(\alpha=.81)$.

Leistungsangst. Die Skala bestand aus neun Items (Itemübersicht siehe Anhang B), mit welchen nach der Gefühlslage während der letzten Klassenarbeit gefragt wurde, wobei vier Items worry-Kognitionen erfassten (Eigenentwicklung vgl. Helmke, 1992; z.B.: „Ich dachte daran, was ich alles nicht kann.") und fünf Items die Wahrnehmung physiologischer Erregung (emotionality) (deutsche Fassung des Test Anxiety Inventory, vgl. Hodapp et al., 1982; z.B.: „Das Herz schlug mir bis zum Hals.“). Das Antwortformat war vierstufig (1 = stimmt gar nicht bis $4=$ stimmt genau). Die interne Konsistenz lag bei $\alpha=.91$.

Kognitive Grundfähigkeit. Es wurde der Subtest N2 des Kognitiven Fähigkeits-Tests für vierte Klassen (KFT 4 R; Heller \& Perleth, 2000) in zwei parallelen Versionen eingesetzt (Version A: $\alpha=.92$, Version B: $\alpha=.93$ ).

\subsubsection{Statistisches Vorgehen}

Die Prüfung der Faktorstruktur der Zielorientierungen wurde mittels konfirmatorischer Faktorenanalysen mit dem Statistikprogramm Mplus 6.1 (Muthén \& Muthén, 1998-2010) vorgenommen. Dabei wurde die Anpassungsgüte von Modellen unterschiedlicher Struktur und Komplexität überprüft. Durch Heranziehung der Anpassungsindizes CFI, TLI, RMSEA und WRMR wurde die relative Güte der ineinander geschachtelten Modelle bestimmt. Folgende Schwellenwerte weisen auf eine sehr gute bzw. gute Modellpassung hin (Yu, 2002): $\mathrm{CFI} \geq .95, \mathrm{TLI} \geq .95, \mathrm{RMSEA} \leq .06$ (bei $\mathrm{N}>250$ ) und WRMR $\leq .95$ bzw. 1.0. Das Modell 1 ging von einer 1-Faktor-Struktur aus. Im Modell 2 folgte die Spezifizierung zweier korrelierender Faktoren (Lern-, Leistungsziele), bevor im Modell 3 die theoretisch postulierte 3Faktor-Struktur mit korrelierenden Faktoren (Lern-, Annäherungsleistungs-, Vermeidungsleistungsziele) spezifiziert wurde. Da es sich um ordinalskalierte Variablen handelt, wurde der Weighted Least-Squares, Mean and Variance-Adjusted-Schätzer (WLSMV) verwendet. Um die geschachtelte Struktur der Daten zu berücksichtigen, wurde der Befehl type = complex verwendet. 
Der Datensatz wies fehlende Werte auf. Bei den Variablen des Schülerfragebogens lag der Anteil bei 17 Prozent, bei den Testleistungen in Mathematik, Naturwissenschaft und den kognitiven Grundfähigkeiten bei unter fünf Prozent. Da die Deutschleistungen nur bei zwei Dritteln der Stichprobe erfasst wurden, betrug der Anteil fehlender Werte 35,8 Prozent. Fehlende Werte wurden mit Hilfe multipler Imputationen (vgl. Graham et al., 2003) unter Einbeziehung weiterer Hintergrundmerkmale (z.B. Geschlecht, Bundesland, Laufbahnempfehlung, soziale Herkunft) geschätzt. Dieses Vorgehen führt zu erwartungstreueren und effizienteren Parameterschätzungen, als wenn Personen mit fehlenden Werten fallweise oder paarweise von den Analysen ausgeschlossen werden (Little \& Rubin, 2002). Um die Prüfung der faktoriellen Validität mit allen Schülern durchführen zu können, wurden fehlende Werte der Zielorientierungen auf Itemebene ersetzt, bei allen anderen Skalen auf Skalenebene. Mit dem Programm Amelia II (Version:1.2-13, Honaker, et al., 2006) wurden fünf vollständige Datensätze erzeugt. Wir berichten jeweils die auf Grundlage der Berechnungsvorschrift von Rubin (1987) von Mplus zu einer Gesamtschätzung kombinierten Ergebnisse.

Da aufgrund der hohen Fallzahlen in Studie 1 auch Korrelationskoeffizienten mit geringer praktischer Relevanz statistisch signifikant wurden, haben wir uns bei der Interpretation der Koeffizienten an Cohen (1988) orientiert: Korrelationskoeffizienten zwischen 0.1 und 0.3 gelten als klein bzw. schwach, zwischen 0.3 und 0.5 als moderat und größer als 0.5 als groß.

\subsubsection{Ergebnisse}

\subsubsection{Faktorstruktur der Zielorientierungen}

Das 1-Faktor-Modell $(\mathrm{CFI}=.77, \mathrm{TLI}=.82, \mathrm{RMSEA}=.21$, WRMR $=8.41)$ und das 2 Faktormodell $(\mathrm{CFI}=.86, \mathrm{TLI}=.90, \mathrm{RMSEA}=.16, \mathrm{WRMR}=6.09)$ erreichten nicht zufriedenstellende Werte. Modell 3 zeigte eine deutliche Verbesserung der Fitstatistiken (CFI = .97, TLI $=.98$, RMSEA $=.07$, WRMR $=2.52)($ siehe Anhang $\mathrm{A}$ für die jeweiligen Faktorladungen). Die latenten Korrelationen betrugen $r=.43, p<.001$ (Lern-/Annäherungsleistungsziele) $r=.72, p<.001$ (Lern-/Vermeidungsleistungsziele) und $r=.53, p<.001$ (Annäherungs-/Vermeidungsleistungsziele). Auf Basis dieser Ergebnisse wurden für die drei Faktoren drei Skalen gebildet, indem die jeweiligen Items rekodiert und für jede Person gemittelt wurden. 


\subsubsection{Deskriptive Befunde}

Die Interkorrelationen zwischen den Zielorientierungen waren positiv und von niedriger bis moderater Größe, wobei sich der höchste Zusammenhang zwischen Lernzielen und der Vermeidungsleistungszielen zeigte (vgl. Tabelle 1). Zur Frage der Konstruktvalidität ergaben sich folgende Ergebnisse: Zum einen korrelierten Lernziele moderat positiv mit dem akademischen Selbstkonzept und der intrinsischen Motivation und nur sehr schwach positiv mit der Leistungsangst. Zum anderen waren die Zusammenhänge zwischen Annäherungsleistungszielen und dem Selbstkonzept sowie der intrinsischen Motivation sehr niedrig, die Beziehung zur Leistungsangst war allerdings niedrig bis moderat positiv. Weiterhin waren die Zusammenhänge zwischen den Vermeidungsleistungszielen und dem Selbstkonzept sowie der intrinsischen Motivation positiv und von einer niedrigen Größenordnung. Die Beziehung zur Leistungsangst war sehr schwach positiv.

\subsubsection{Zusammenhang zwischen Zielorientierungen und Schulleistungen}

Es ergaben sich für die Lernziele Korrelationen nahe Null mit den Testleistungen und sehr schwache negative Korrelationen mit den Noten (vgl. Tabelle 1). Die Größenordnung der Zusammenhänge zwischen Vermeidungsleistungszielen und den Schulleistungen lag bei Null. Allein Annäherungsleistungsziele wiesen niedrige Zusammenhänge mit den Noten und den Testleistungen auf: Je höher die Annäherungsleistungsziele, desto niedriger die Testleistungen und desto schlechter die Schulnoten in allen betrachteten Domänen bzw. Fächern. Für Mathematik war die Korrelation mit den Testleistungen etwas höher als mit den Noten, während sich für Deutsch die Koeffizienten kaum unterschieden. 
Tabelle 1

Deskriptive Statistiken und Interkorrelationen (Produkt-Moment-Korrelationen)

\begin{tabular}{|c|c|c|c|c|c|c|c|c|c|c|c|c|c|c|}
\hline & $M$ & $S D$ & 2 & 3 & 4 & 5 & 6 & 7 & 8 & 9 & 10 & 11 & 12 & 13 \\
\hline ALZ (2) & 2.65 & 0.84 & & .33 & -.22 & -.26 & -.23 & .16 & .25 & .19 & .05 & .13 & .25 & -.23 \\
\hline VLZ (3) & 3.51 & 0.64 & & & -.01 & .01 & .00 & -.02 & -.00 & -.01 & .18 & .20 & .07 & -.02 \\
\hline Testleistung Mathematik (4) & 150 & 10 & & & & .58 & .61 & -.63 & -.59 & -.52 & .27 & .01 & -.34 & .50 \\
\hline Note Mathematik (7) & 2.74 & 0.97 & & & & & & & .67 & .65 & -.42 & -.09 & .35 & -.44 \\
\hline Note Deutsch (8) & 2.73 & 0.91 & & & & & & & & .70 & -.41 & -.12 & .34 & -.41 \\
\hline Note Sachunterricht (9) & 2.49 & 0.90 & & & & & & & & & -.38 & -.10 & .32 & -.38 \\
\hline Selbstkonzept (10) & 3.38 & 0.57 & & & & & & & & & & .40 & -.25 & .17 \\
\hline Kognitive Grundfähigkeit (13) & 15 & 7 & & & & & & & & & & & & \\
\hline
\end{tabular}

Anmerkungen. LZ: Lernziele. ALZ: Annäherungsleistungsziele. VLZ: Vermeidungsleistungsziele. Schulnoten: $1=$ sehr gut bis $6=$ ungenügend. Korrelationskoeffizienten $>.05$ sind statistisch signifikant $(p<.001)$. 
Um den unabhängigen Effekt der einzelnen Zielorientierungen zu bestimmen, wurden multiple lineare Regressionsanalysen mit den Zielorientierungen und der kognitiven Grundfähigkeit als Prädiktoren und den Schulleistungen als Kriterium berechnet. Die kognitive Grundfähigkeit wurde in die Analysen mit aufgenommen, da diese allgemein als relevanter Prädiktor von Schulleistungen gilt. Der negative Effekt der Annäherungsleistungsziele auf die schulischen Leistungen blieb bestehen (Tabelle 2). Weiterhin ergab sich für die Deutschleistung ein schwach positiver Effekt der Vermeidungsleistungsziele. Für die Mathematik- und Sachunterrichtsnote entsprachen die Ergebnisse weitestgehend denen der Deutschnote (ohne Tabelle). Aufgrund des Zusammenhangs zwischen Annäherungs- und Vermeidungsleistungszielen mit Leistungsangst wurde abschließend in einer multiplen Regression geprüft, ob der negative Effekt der Leistungsziele auf die Schulleistungen über die Leistungsangst vermittelt war. Die Befunde wiesen auf eine partielle Vermittlung hin (ohne Tabelle). 
Tabelle 2

Zusammenfassung der multiplen linearen Regression zur Vorhersage der schulischen Leistungen (standardisierte Koeffizienten)

\begin{tabular}{|c|c|c|c|c|c|c|c|c|}
\hline & \multicolumn{2}{|c|}{$\begin{array}{l}\text { Testleistung } \\
\text { Naturwissenschaft }\end{array}$} & \multicolumn{2}{|c|}{$\begin{array}{l}\text { Testleistung } \\
\text { Mathematik }\end{array}$} & \multicolumn{2}{|c|}{$\begin{array}{l}\text { Testleistung } \\
\text { Deutsch }\end{array}$} & \multicolumn{2}{|l|}{$\begin{array}{l}\text { Note } \\
\text { Deutsch }\end{array}$} \\
\hline & $\beta$ & $S E$ & $\beta$ & $S E$ & $\beta$ & $S E$ & $B$ & $S E$ \\
\hline $\mathrm{LZ}$ & -.02 & .02 & -.01 & .02 & .02 & .02 & -.00 & .02 \\
\hline ALZ & $-.14 * * *$ & .02 & $-.11 * * *$ & .02 & $-.17 * * *$ & .02 & $.14^{* * *}$ & .01 \\
\hline VLZ & .04 & .02 & .02 & .02 & $.06 * * *$ & .02 & -.02 & .02 \\
\hline Kognitive Grundfähigkeit & $.30 * * *$ & .01 & $.40 * * *$ & .01 & $.28 * * *$ & .02 & $-.25 * * *$ & .01 \\
\hline $\mathrm{R}^{2}$ & $.27 * * *$ & & $.35 * * *$ & & $.28 * * *$ & & $.37 * * *$ & \\
\hline
\end{tabular}

Anmerkungen. LZ: Lernziele. ALZ: Annäherungsleistungsziele. VLZ: Vermeidungsleistungsziele.SE = Standardfehler. Deutschnote: $1=$ sehr gut bis $6=$ ungenügend.

$* * * p<.001$. 


\subsubsection{Diskussion Studie 1}

Das Ziel von Studie 1 war es, neben der faktoriellen Struktur und der Konstruktvalidität von Zielorientierungen, den Zusammenhang mit Schulleistungen am Ende der Grundschulzeit zu untersuchen. Die Analyse der Faktorstruktur führte zu einer empirischen Bestätigung der theoretisch postulierten 3-Faktorstruktur. Die Zielorientierungen ließen sich faktoriell valide und reliabel messen. Demgegenüber wiesen die Analysen auf eine eingeschränkte Konstruktvalidität der PALS hin. Während die Validitätskoeffizienten für die Lernziele den Erwartungen vor allem im Hinblick auf Selbstkonzept und Motivation entsprachen, war dies für die Vermeidungsleistungsziele nicht der Fall. Diese scheinen am Ende der Grundschulzeit noch nicht Ausdruck eines dysfunktionalen lernbezogenen Verhaltensmusters zu sein, das konsistent mit Merkmalen eines ungünstig verlaufenden Lernprozesses (z.B. einem niedrigen Fähigkeitsselbstkonzept, einer erhöhten Leistungsangst) in Verbindung gebracht werden kann (Helmke, 1992). Betrachtet man den positiven Zusammenhang mit der Leistungsangst, so scheint dies eher für die Annäherungsleistungsziele zuzutreffen.

Im Hinblick auf die Schulleistungen zeigte sich allein mit den Annäherungsleistungszielen ein praktisch bedeutsamer negativer Zusammenhang. Dieser Befund kam unerwartet, da diese Form der Zielorientierung in der bisherigen Forschung zumindest in einen begrenzt positiven Zusammenhang mit den Schulleistungen gebracht werden konnte. Die Lernziele waren wider Erwarten kaum prädiktiv für die Schulleistungen. Für den unerwarteter Weise nicht signifikanten negativen Zusammenhang zwischen Vermeidungsleistungszielen und Schulleistung mag die geringe Validität dieses Merkmals für ein dysfunktionales lernbezogenes Verhaltensmuster verantwortlich sein. 


\subsection{Studie 2}

Um auszuschließen, dass es sich bei den in Studie 1 gefundenen Ergebnissen um für das Instrument spezifische Befunde handelt, haben wir in Studie 2 mittels eines im deutschen Sprachraum erprobten Instruments zur Erfassung von Zielorientierungen (SELLMO) dieselbe Fragestellung untersucht. Dabei ist von besonderem Interesse, ob sich der negative Zusammenhang zwischen Annäherungsleistungszielen und den Schulleistungen an einer anderen Stichprobe und auf Basis eines anderen Instruments replizieren lässt.

\subsubsection{Methode}

Dazu wurden die Daten einer Übergangsstudie analysiert (vgl. Aust et al., 2010), bei welcher in der vierten Woche nach dem Übergang in die fünfte Jahrgangsstufe die Schulleistungen von Schülern mit objektiven Leistungstests und Ziffernnoten sowie deren Zielorientierungen erfasst wurden.

\subsubsection{Stichprobe}

An der Untersuchung nahmen $N=294$ Schülerinnen (51,7 \%) und Schüler des fünften Jahrgangs eines Gymnasiums (fünf Klassen, $n=139$ ) und einer Integrierten Gesamtschule (sechs Klassen, $n=155$ ) einer mittelgroßen deutschen Universitätsstadt teil.

\subsubsection{Instrumente und Operationalisierung}

Zielorientierungen. Es wurden die SELLMO (Spinath et al., 2002) eingesetzt. Der Fragebogen besteht aus unabhängigen Skalen für die Messung von Lern- und Annäherungsbzw. Vermeidungsleistungszielen und Arbeitsvermeidung. Für die nachfolgenden Analysen wurden die Lern- (acht Items, $\alpha=.86$, Beispielitem: „In der Schule geht es mir darum, etwas Interessantes zu lernen.") sowie die Annäherungsleistungs- (sieben Items, $\alpha=.86$, Beispielitem: „In der Schule geht es mir darum, bessere Noten oder Beurteilungen zu bekommen als andere.“) und die Vermeidungsleistungsziele (acht Items, $\alpha=.91$, Beispielitem: „In der Schule geht es mir darum, zu verbergen, dass ich weniger weiß als andere.") verwendet (Itemübersicht siehe Anhang C). Die Items waren auf einer 5-stufigen Ratingskala von stimmt gar nicht bis stimmt genau einzuschätzen. 
Schulleistungen. Die Mathematikleistung wurde mit insgesamt 16 Aufgaben der Hamburger Studie zu „Kompetenzen und Einstellungen von Schülern“ am Ende der Jahrgangsstufe vier (KESS 4; vgl. Bos \& Pietsch, 2005), sowie des Forschungsprojekts „Bildungsverläufe und psychosoziale Entwicklung im Jugend- und jungen Erwachsenenalter“ (BIJU; vgl. Baumert et al., 1996) erfasst. Es handelte sich dabei mehrheitlich um Multiple-Choice-Aufgaben, die durch Aufgaben mit offenem Antwortformat ergänzt wurden. Das Instrument wies ein $\alpha$ von .73 auf.

Zur Erfassung der Leseleistung wurden 16 Aufgaben aus der Internationalen Grundschul-Lese-Untersuchung (IGLU; vgl. Bos et al., 2004) sowie dem Diagnostischen Test Deutsch (Nauck \& Otte, 1980) verwendet. Auch hierbei waren mehrheitlich Multiple-ChoiceAufgaben zu bearbeiten, die anderen Fragen hatten ein offenes Antwortformat $(\alpha=.72)$.

Sowohl der Mathematik- als auch der Lesetest wurden mit dem Computerprogramm ConQuest (vgl. Wu, Adams \& Wilson, 1998) raschskaliert und Itemparameter und WeightedLikelihood-Estimates (WLE; Warm, 1989) als Personenparameter geschätzt (Metrik von $M=$ 100 und $S D=30)$.

Zusätzlich zu den Testleistungen wurden die Endjahresnoten (Klasse vier) in Deutsch, Mathematik und Sachunterricht bei den Schülern des Gymnasiums erhoben. An der Integrierten Gesamtschule war es nicht möglich, diese Informationen zu erhalten.

Kognitive Grundfähigkeit. Die verbale und figurale kognitive Grundfähigkeit wurde mit dem Kognitiven Grundfähigkeitstest für fünfte Klassen (Heller \& Perleth, 2000) erfasst.

\subsubsection{Statistisches Vorgehen}

Die Analysen wurden mit dem Statistikprogramm Mplus 6.1 (Muthén \& Muthén, 1998-2010) durchgeführt. Fehlende Werte (Zielorientierungen: 11,9 \%; Lesetest: 4,2 \%; Mathematiktest: 10,7 \%; Noten: $60 \%$, da nur Angaben vom Gymnasium) wurden auf Basis der Full Information Maximum Likelihood Methode geschätzt.

\subsubsection{Ergebnisse}

Betrachten wir zunächst die Beziehungen zwischen den Zielorientierungen (Tabelle 3). Die beiden Facetten der Leistungsziele korrelierten am höchsten miteinander. Die Lernziele waren mit den Annäherungsleistungszielen gering bis moderat sowie mit den Vermeidungsleistungszielen unkorreliert. Im Hinblick auf die Schulleistungen ergaben sich differen- 
tielle Ergebnisse für Testleistungen und Noten: Insbesondere korrelierten die Leistungen im Lesen und in Mathematik schwach negativ mit den Annäherungsleistungszielen. Die Vermeidungsleistungsziele waren erwartungsgemäß negativ korreliert mit der Leseleistung, nicht jedoch mit der Mathematikleistung. Für das Lesen zeigte sich auch ein erwarteter positiver Zusammenhang mit den Lernzielen. Die Sachunterrichtsnote zeigte den größten Zusammenhang mit beiden Dimensionen der Leistungsziele. Bei der Betrachtung der Zusammenhänge zwischen Zielorientierungen und Schulnoten ist zu berïcksichtigen, dass die Schulnoten nur am Gymnasium erhoben wurden. Die differentiellen Befunde bleiben allerdings robust, auch wenn man die Zusammenhänge mit den Testleistungen nur für das Gymnasium berechnet.

Tabelle 4 gibt die Ergebnisse der multiplen linearen Regression der Schulleistungen auf die Zielorientierungen und die kognitive Grundfähigkeit wieder. Kein Effekt der Zielorientierungen auf die Noten wird signifikant und die kognitive Grundfähigkeit leistet als einzige Variable einen signifikanten Erklärungsbeitrag. Bei den Testleistungen war der Effekt der Lernziele auf die Leseleistung positiv. Der Effekt der Annäherungsleistungsziele auf die Lese- und Mathematikleitsung war negativ. 
Tabelle 3

Deskriptive Statistiken und Interkorrelationen (Produkt-Moment-Korrelationen)

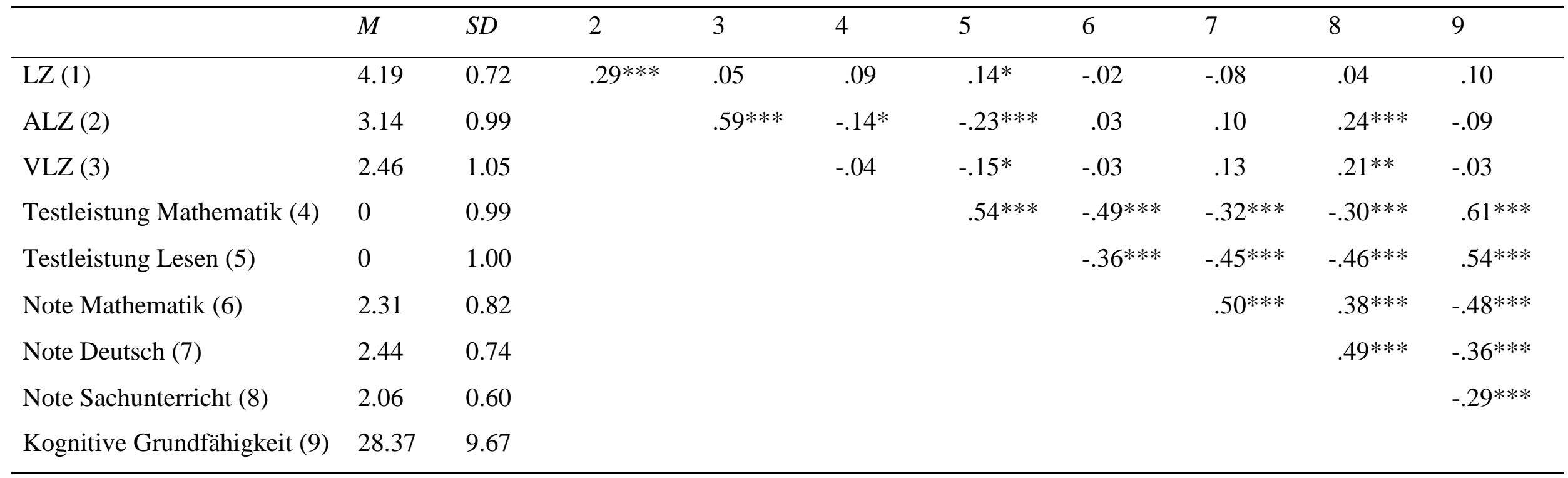

Anmerkungen. LZ: Lernziele. ALZ: Annäherungsleistungsziele. VLZ: Vermeidungsleistungsziele. $N=294$. Schulnoten $(1=$ sehr gut bis $6=$ ungenügend) wurden nur am Gymnasium erhoben $(n=139)$.

$* p<.05 . * * p<.01 . * * * p<.001$. 
Tabelle 4

Zusammenfassung der multiplen linearen Regression zur Vorhersage der schulischen Leistungen (standardisierte Koeffizienten)

\begin{tabular}{|c|c|c|c|c|c|c|c|c|c|c|}
\hline & \multicolumn{2}{|c|}{$\begin{array}{l}\text { Testleistung } \\
\text { Lesen }\end{array}$} & \multicolumn{2}{|c|}{$\begin{array}{l}\text { Testleistung } \\
\text { Mathematik }\end{array}$} & \multicolumn{2}{|l|}{$\begin{array}{l}\text { Note } \\
\text { Deutsch }\end{array}$} & \multicolumn{2}{|c|}{$\begin{array}{l}\text { Note } \\
\text { Mathematik }\end{array}$} & \multicolumn{2}{|c|}{$\begin{array}{l}\text { Note } \\
\text { Sachunterricht }\end{array}$} \\
\hline & $\beta$ & $S E$ & $\beta$ & $S E$ & $\beta$ & $S E$ & $\beta$ & $S E$ & $\beta$ & $S E$ \\
\hline $\mathrm{LZ}$ & $.16^{* * *}$ & .05 & .06 & .05 & -.03 & .08 & .04 & .08 & .02 & .08 \\
\hline ALZ & $-.24 * * *$ & .06 & $-.14^{*}$ & .06 & -.00 & .10 & -.01 & .09 & .13 & .10 \\
\hline VLZ & .00 & .06 & .06 & .06 & .11 & .10 & -.05 & .10 & .12 & .10 \\
\hline Kognitive Grundfähigkeit & $.52 * * *$ & .04 & $.60 * * *$ & .04 & $-.33 * * *$ & .08 & $-.47 * * *$ & .07 & $-.30 * * *$ & .08 \\
\hline $\mathrm{R}^{2}$ & $.36 * * *$ & & $.39 * * *$ & & $.13^{*}$ & & $.22 * * *$ & & $.15 * *$ & \\
\hline
\end{tabular}

Anmerkungen. LZ: Lernziele. ALZ: Annäherungsleistungsziele. VLZ: Vermeidungsleistungsziele. $N=294$. Schulnoten $(1=$ sehr gut bis $6=$ ungenügend) wurden nur am Gymnasium erhoben $(n=139)$. SE $=$ Standardfehler.

$* p<.05 . * * p<.01 . * * * p<.001$. 


\subsubsection{Diskussion Studie 2}

Das Ziel von Studie 2 war, die in Studie 1 gefundenen teils unerwarteten Befunde anhand eines für den deutschen Sprachraum etablierten Instruments zur Erfassung der Zielorientierungen zu replizieren. Von besonderem Interesse war dabei, ob sich der negative Zusammenhang zwischen Annäherungsleistungszielen und Schulleistung auch in Studie 2 finden lässt. Die Ergebnisse bestätigten diesen Zusammenhang, wobei sich der Befund bei Kontrolle der anderen Dimensionen der Zielorientierung und der kognitiven Grundfähigkeit für die Noten nicht mehr zeigte. Im Unterschied zu Studie 1 konnte zum einen die Hypothese bestätigt werden, dass Vermeidungsleistungsziele - bei bivariater Betrachtung - negativ mit Schulleistungen in Beziehung stehen, zum anderen fand sich für die Leseleistung ein positiver Zusammenhang mit den Lernzielen. Die differentiellen Befunde für Noten und Testleistungen sollen hier mit Vorsicht betrachtet werden, da diese zum einen nur für das Gymnasium betrachtet werden konnten und zum anderen bei der Benotung mit Referenzgruppeneffekten gerechnet werden muss.

\subsection{Gesamtdiskussion}

Die vorliegende Arbeit untersuchte den Zusammenhang zwischen Zielorientierungen und Schulleistungen am Grundschulübergang. Im Zentrum stand die Frage, ob Zielorientierungen bei Schülern dieser Altersstufe faktoriell valide und reliabel gemessen werden können und in welchem Zusammenhang diese mit den Schulleistungen stehen. Weiterhin war von Interesse, ob es differentielle Befunde bei der Verwendung von Leistungen in standardisierten Tests oder von Ziffernnoten gibt. Im Unterschied zu bisherigen Studien basierten die Analysen von Studie 1 auf einer für Länder mit vierjähriger Primarstufe repräsentativen Stichprobe von Viertklässlern. In Studie 2 erfolgte eine Replikation der Befunde mit den SELLMO.

Ein erster Befund war, dass Zielorientierungen mit der modifizierten Kurzversion der PALS faktoriell valide und reliabel gemessen werden können. Trotz der geringen Anzahl von Items (besonders für die Vermeidungsleistungsziele) lagen die Reliabilitäten in einem zufriedenstellenden bis sehr guten Bereich. Damit fügen sich die Ergebnisse gut in den erst kürzlich in einer Metaanalyse von Hulleman et al. (2010) vorgestellten Befund ein, dass Zielorientierungen bereits im Primarbereich reliabel gemessen werden können. Ein zweiter Befund wies auf eine eingeschränkte Konstruktvalidität des eingesetzten Instruments im Bereich der Vermeidungsleistungsziele hin. Sowohl der unerwartet moderat positive Zusammenhang mit den 
Lernzielen wie auch die erwartungswidrig positiven Zusammenhänge mit dem Selbstkonzept und der Motivation deuteten darauf hin, dass die Indikatoren der Vermeidungsleistungsziele (möglicherweise noch) nicht als Ausdruck eines dysfunktionalen lernbezogenen Erlebensund Verhaltensmusters aufzufassen sind. Beim Einsatz der SELLMO in Studie 2 deuteten sowohl die Interkorrelationen der Zielorientierungen wie auch die Zusammenhänge mit den Schulleistungen auf eine befriedigendere Konstruktvalidität der Vermeidungsleistungsziele hin. Letztlich bleibt hier jedoch weiterer Forschungsbedarf, da die zur Studie 1 abweichenden Befunde auf das Instrument bzw. auf die Facetten zur Erfassung der Zielorientierungen (vgl. Hulleman et al., 2010), auf Spezifika der Stichprobe oder der Tests zur Erfassung der Schulleistungen zurückgehen können.

Ein dritter Befund betraf den Zusammenhang mit den Schulleistungen. Hier zeigte sich in beiden Studien ein negativer Zusammenhang zwischen Annäherungsleistungszielen und der Schulleistung. Dieser Befund kam unerwartet, da in den meisten bisherigen Untersuchungen diese Form der Zielorientierung entweder keinen (z.B. Finsterwald, 2006) oder einen positiven (z.B. Bong, 2009) Zusammenhang mit den Schulleistungen zeigte. Wie die Richtung des Zusammenhangs dabei aussieht, kann wegen des Querschnittdesigns empirisch nicht geklärt werden. Eine höhere Annäherungsleistungszielorientierung kann zum einen die niedrigeren schulischen Leistungen bedingen, möglicherweise über eine verstärkte Nutzung von Oberflächenstrategien bzw. einen verringerten Einsatz von Tiefenverarbeitungsstrategien. Sie kann zum anderen aber auch Folge der Leistungsbiographie eines Kindes sein (Brophy, 2005). Allerdings würde man bei letzterem - auf Basis erwartungs-mal-wert-theoretischer Überlegungen - einen positiven Zusammenhang zwischen Leistungen und Annäherungsleistungszielen erwarten, d.h. es sollten die leistungsstärkeren Schüler mit höheren subjektiven Erfolgserwartungen sein, die sich verstärkt auch Annäherungsleistungsziele im Unterricht setzen. Da dies nicht der Fall ist, argumentieren wir daher eher für eine die Kompetenzentwicklung hemmende Rolle von Annäherungsleistungszielen in der Grundschule, wie es bereits Finsterwald, Ziegler und Dresel (2009) geschlussfolgert haben. Der positive Zusammenhang zwischen Annäherungsleistungszielen und Leistungsangst deutet darauf hin, dass auch negative Emotionen hier nicht unbedeutend sind. Und in der Tat war der negative Effekt der Vermeidungsleistungsziele partiell durch die Leistungsangst vermittelt. Die Beziehungen zwischen Lernzielen und Schulleistungen waren sehr schwach und meist nicht signifikant. Dieses Ergebnis fügt sich in die Befundlage anderer Untersuchungen an dieser Altersgruppe ein (Bong, 2009; Finsterwald, 2006). Eine mögliche Erklärung dafür, dass Lernziele in diesem Alter noch keinen substanziellen positiven Zusammenhang mit den Schulleistungen auf- 
weisen, könnte darin bestehen, dass Viert- bzw. Fünftklässler vermehrt Oberflächenstrategien nutzen (Hübner, Nückles \& Renkl, 2010) und sich daher die Wirkung des mehr oder weniger effektiven Einsatzes von Tiefenverarbeitungsstrategien auf die Schulleistungen noch nicht zeigen kann. Shim, Ryan und Anderson (2008) argumentieren, dass sich Lernziele möglicherweise deshalb erst in der weiterführenden Schule positiv auf die Schulleistungen auswirkt, weil die Lernumwelten dort herausfordernder seien und ein höheres Maß an Eigenverantwortung abverlangt wird. Allerdings gibt es in der Literatur auch die Position, dass Lernziele weniger Einfluss auf die Leistung, sondern stattdessen auf die Motivation nehmen (Schiefele, 2009). Unser Ergebnis aus Studie 1, in welcher ein straffer Zusammenhang zwischen Lernzielen und intrinsischer Motivation gefunden wurde, stützt dieses Argument.

Inhaltlich sprechen die Befunde beider Studien für einen negativen Zusammenhang zwischen Annäherungsleistungszielen und Schulleistungen am Ende der vierten bzw. zu Beginn der fünften Klassenstufe. Weitere Forschung müsste die Vermittlungsprozesse zwischen Zielorientierungen und Schulleistungen vertiefend analysieren. So wäre etwa zu untersuchen, inwieweit sich Schüler mit unterschiedlichen Zielorientierungen tatsächlich in der Anwendung von Lernstrategien oder in leistungsrelevanten motivationalen und emotionalen Merkmalen unterscheiden. Für die pädagogische Praxis könnte sich die Forderung ableiten, im Unterricht darauf hinzuwirken, dass Annäherungsleistungsziele weniger bedeutsam werden (vgl. Schunk et al., 2008).

Schließlich sind wir der Frage differentieller Befunde bei der Verwendung von Noten und Testleistungen nachgegangen. Belastbare Ergebnisse lieferte hier Studie 1, in der die Lernziele ein wenig enger mit den Noten und die Annäherungsleistungsziele ein wenig enger mit der Mathematikleistung zusammenhingen. Allerdings waren die Unterschiede von geringer praktischer Relevanz.

Abschließend möchten wir einige Grenzen unserer Untersuchung diskutieren. Zum einen wurden die Zielorientierungen bereichsunspezifisch erfasst. Daher ist nicht auszuschließen, dass eine bereichsspezifische Erfassung zu einem differentiellen Zusammenhangsmuster mit den Schulleistungen geführt hätte. Zum anderen wurden in Studie 2 lediglich zwei Schulen (Gymnasium, integrierte Gesamtschule) betrachtet, was bei der Interpretation der Ergebnisse zu bedenken ist. Da die Daten zudem kurze Zeit nach dem Übergang auf die weiterführende Schule erhoben wurden, kann nicht ausgeschlossen werden, dass der Bezugsgruppenwechsel einen Einfluss auf die Ergebnisse hatte. Außerdem basieren die Befunde beider Untersuchungen auf Querschnittdaten, sodass letztlich über die Richtung der gefundenen $\mathrm{Zu}$ sammenhänge nur spekuliert werden kann. Es fehlen Längsschnittstudien in der Grundschule 
bzw. beim Übergang in die weiterführende Schule, in denen der Effekt der Zielorientierungen auf die Leistungsentwicklung untersucht werden kann.

Generell kann man aus unseren Befunden lernen, dass sich Zielorientierungen am Grundschulübergang durchaus reliabel erfassen lassen, dass allerdings die lernpsychologische Bedeutung dieser Konstrukte teilweise offenbar eine andere ist als bei älteren Schülern oder Erwachsenen. Die Ergebnisse zu den Annäherungsleistungszielen lieferten diesbezüglich unerwartete und zugleich eindrucksvolle Belege. 


\section{Achievement goals and school achievement: The transition to different school tracks in secondary school (Studie 3) ${ }^{10}$}

\subsection{Abstract}

The transition from elementary to secondary school can be a source of change in the association between achievement goals and achievement. In Germany, early adolescents are assigned to one of two school tracks, academic or non-academic, that differ markedly in compositional and institutional characteristics. Currently, there is no research examining both the development of achievement goals and the changes in the association between achievement goals and achievement during the transition to these different school tracks. $N=1,646$ students participated in a large-scale, three-wave longitudinal study from grade four to grade six. While results revealed only slight differences between the two school tracks, the three types of achievement goals declined over time. Mastery goals predicted achievement positively, whereas performance-approach goals negatively influenced achievement development (academic tracks). Furthermore, performance-approach goals turned out to be detrimental for young students's school achievement before and after the transition (academic track).

\subsection{Introduction}

The transition from elementary to secondary school, an important and critical event in the life of every child, is often associated with a decline in motivation (e.g., Anderman \& Maehr, 1994; Eccles \& Midgley, 1989). Achievement goals are considered part of motivation because they pertain to self-motivational beliefs in the forethought phase of the cyclical model of self-regulation (Zimmerman, 1998; Zimmerman \& Campillo, 2003) and accordingly refer to processes that precede and prepare self-regulated actions and successful learning.

Although the development of achievement goals has often been investigated during the transition from primary to secondary school, the transition into different school tracks has rarely been studied. The German school system differs from those in Anglo-Saxon countries as the majority of the students are assigned to one of three different school tracks: Hauptschule (low track), Realschule (middle track) or Gymnasium (academic track). Each of these

\footnotetext{
${ }^{10}$ Dieser Abschnitt basiert auf einem bei der Zeitschrift Contemporary Educational Psychology eingereichten Manuskript (Paulick, Watermann \& Nückles, 2011a).
} 
tracks differs markedly from the others in the ability level of the students and the learning environments of the classes. Accordingly, the primary purpose of the current study is to examine the development of achievement goals and the changes in the association between achievement goals and achievement during the transition to different school tracks in secondary school.

\subsection{Achievement goals}

Achievement goals are defined as cognitive representations of competence-relevant possibilities that guide behavior to a competence-related end state that the individual is committed to either approach or avoid (Elliot \& Thrash, 2001; Hulleman et al., 2010). These goals are associated with behavioral and performance variability (McKinney, 2003). Achievement goals are conceptualized as dynamic and changing in relation to the context (Shim et al., 2008).

They are divided into two types of goals that individuals pursue in task/learning contexts: mastery and performance goals (Ames \& Ames, 1984). A mastery goal is the desire to acquire new skills, master new situations, and improve one's competence. Conversely, students with high performance goals seek to demonstrate or prove one's competence to others. Additionally, the distinction between approach and avoidance is made for both mastery and performance goals (Elliot, 1999; Elliot \& McGregor, 2001). Mastery-approach goals are focused on attaining task-based or intrapersonal competence, whereas mastery-avoidance goals are focused on avoiding task-based or intrapersonal incompetence. However, there is little research on mastery-avoidance goals. Attaining normative competence, demonstrating high competence, and gaining positive judgments from others is part of performance-approach goals, whereas avoiding normative incompetence and preventing negative judgments from others is part of performance-avoidance goals (Elliot, 1999).

\subsection{The development of achievement goals}

According to Nicholls $(1984,1990)$, there is an increase in performance goals, which is caused by the cognitive development of the students. He justifies this with the assumption that students have an undifferentiated concept of ability until the end of primary school, and they do not distinguish between effort and ability or make many social comparisons. Rather, they have an individual frame of reference. Children in this stage of development are, per de- 
finition, mastery-goal oriented. During their cognitive development, their concept of ability becomes increasingly differentiated. Students are now able to distinguish between effort and ability and tend to compare their own performance to others. When the same level of performance is obtained with less effort, students now begin to infer higher ability. These changes in the concept of ability and the more salient processes of social comparison make an increase in performance goals more likely.

Another theoretical perspective to explain the development of achievement goals is Dweck's (2000) approach. In her implicit theory of intelligence, individuals hold self-theories regarding whether their personal qualities are fixed or malleable. These self-theories direct them into different behaviors and outcomes. Most young children are incremental theorists; that is, they believe intelligence can be improved by investing effort. For them, it is more important to acquire new competence than to validate their competence. In contrast, children with an entity theory believe that intelligence is something one either possesses or does not possess. For them, it is important to validate their competence. Accordingly, an entity theory is associated with performance goals, whereas an incremental theory is associated with mastery goals. By the time children advance to upper school grades, they are more likely to develop an entity theory of intelligence. Thus, their performance goals should increase.

From a contextual perspective, some researchers suggest that middle-level schools can be a major source of decline in motivation during early adolescence (Eccles, Lord, Roeser, Barber \& Jozefowicz, 1997; Juvonen, Le, Kaganoff, Augustine \& Constant, 2004). The changes in classroom organization, instruction, and climate variables contribute to the decline in student motivation or rather in student achievement goals. As students grow older, their needs change. The desire for autonomy and self-determination as well as peer orientation and identity issues become more important. In light of these needs, the environmental changes that are often associated with the transition to secondary school, such as an emphasis on competition, social comparison, and control, seem especially harmful (Eccles et al., 1993). These changes could result in a developmental mismatch between early adolescent needs and classroom environment, which may cause a decline in achievement goals as a motivational outcome (stage-environment fit; Eccles et al., 1993). This decline might be especially salient for mastery goals as an expression of motivation, while performance goals may increase because they are more likely to be negatively related to motivational outcomes. In a German study by Daniels (2008), a decline in student interest from grade seven to grade ten was found. As the transition from elementary to secondary school occurs in most Anglo-Saxon countries after 
grade six, Daniels' findings referred to students of the same age and can be interpreted as a result of a possible developmental mismatch.

There are several bodies of research on the development of achievement goals during the transition to secondary school, especially in Anglo-Saxon countries (e.g. Anderman \& Midgley, 2004; Eccles \& Midgley, 1990), that reveal inconsistent results. For example, one line of study found a decrease in mastery and an increase in performance goals (e.g., Anderman \& Anderman, 1999; Midgley, Anderman \& Hicks, 1995). However, a second line found a general decline in all types of achievement goals (e.g., Bong, 2009; Shim et al., 2008). Because the transition from elementary to secondary school occurs after grade four in Germany, the results of the presented studies cannot be easily applied to the German school system. There are only a few German studies on the development of achievement goals (Finsterwald, 2006; Köller, 1998; Schwinger \& Wild, 2006). Schwinger and Wild (2006) examined subjectspecific achievement goal profiles in mathematics $(N=208)$ from grade three to grade five, a case where the transition to secondary school occurred after grade four. Their results could not confirm Nicholls' assumptions of a change from mastery to performance goals. Rather, they found a low stability in student goal profiles over time and a small decline in all three types of achievement goals. Finsterwald (2006) also found a decline in all types of achievement goals during the transition from elementary school to secondary school. Köller (1998) examined the development of achievement goals of $N=1,941$ students in different school tracks in grade seven and found that students in academic tracks were less performance-goal oriented than students in non-academic tracks. However, these findings cannot be generalized to younger students during the transition to secondary school.

Most of the presented studies used the Patterns of Adaptive Learning Scales (PALS; Midgley \& Maehr, 1993; Midgley et al., 1995, 2000) to measure achievement goals. Studies (Anderman \& Anderman, 1999; Midgley et al., 1995) that used the original version of the PALS (Midgley \& Maehr, 1993; Midgley et al., 1998) often showed a decline in mastery goals and an increase in performance goals, whereas studies (e.g., Bong, 2009; Finsterwald, 2006; Shim et al., 2008) that used the revised version of the PALS (Midgley et al., 2000) or another instrument to measure achievement goals (e.g., Schwinger \& Wild, 2006 used, among others, a German version of the Motivational Orientation Scales by Köller \& Baumert, 1998; original version by Nicholls et al., 1985) found an overall decline in achievement goals. The inconsistent findings of the current research can be caused by conceptual and measurement differences between the different studies. 


\subsection{The relation between achievement goals and school achievement}

The relation between achievement goals and school achievement was often explained with the use of certain learning strategies (e.g., Bong, 2009; Elliot, et al., 1999; Köller, 1998). Thus, mastery-goal oriented students more often use deep-learning strategies, whereas performance-goal oriented students, not differentiated between approach and avoidance, more frequently use surface learning strategies (e.g., Bouffard et al., 1995; Harackiewicz et al., 2000). In turn, the use of certain learning strategies can have a positive effect on school learning and achievement (Bandalos et al, 2003; Pintrich \& De Groot, 1990). Accordingly, the relation between achievement goals and school achievement could be mediated by the use of learning strategies (Fenollar, Román \& Cuestas, 2007; Greene, Miller, Crowson, Duke \& Akey, 2004, Harackiewicz et al., 2000).

Other variables that showed differences according to achievement goals were emotions. Pekrun and colleagues underlined the role of emotions and achievement goals for school learning. Mastery goals were related to positive emotions, such as enjoyment whereas performance-avoidance goals were related to negative emotions, such as anxiety. Emotions, in turn, have found to be related to school achievement (Pekrun et al., 2006).

Most of the studies on mediating effects between achievement goals and school achievement referred to undergraduates (e.g., Diseth \& Kobbeltvedt, 2010; Elliot \& McGregor, 2001; Fenollar et al., 2007; Pekrun et al., 2006) while only a few studies focused on younger students and those were on students in grade six or junior high school (Bong, 2009; Pintrich, 2000b; Pintrich \& De Groot, 1990; Wolters, 2004). There are currently no known studies on students in grade five or below that investigated the mediating role of certain variables for the relation between achievement goals and school achievement. However, the results of the present are not easily transferable to elementary school students.

Hulleman, Schrager, Bodmann and Harackiewicz (2010) reviewed 243 correlational studies of achievement goals, with only 20 elementary school studies, and found that conceptual and measurement differences between the different studies moderated the association between achievement goals and achievement outcomes. They found a positive correlation between performance-approach goals and performance outcomes when the goal scales consisted of normative items, whereas there was a negative correlation when the goal scales consisted of appearance and evaluative items. The results for mastery goals revealed that there was no relation to achievement when the goal scales contained goal-relevant language, for example, "An important reason why I do my class work is because I like to learn new things". 
However, there was a positive correlation when the scales did not contain goal-relevant language, for example, "I like learning new things from physical education, even if I make mistakes" (Hulleman et al., 2010, pp. 431). The results for performance-avoidance goals have consistently been found to be negatively associated with achievement. Thus, the goal scale chosen, the individual items used to assess (task, potential improvement for mastery; normative and appearance for performance-approach; appearance and fear for performanceavoidance), and the socio-demographic characteristics of the sample (e.g., nationality) influenced the results of the studies. However, only a small number of the reviewed studies included elementary or secondary school students.

Other studies on the relation between achievement goals and achievement have yielded inconsistent findings. Some international researchers, for example, have found a positive association between mastery/performance-approach goals and achievement (e.g., Elliot, Shell, Bouas, Henry \& Maier, 2005; Ryan et al., 2005), while others found no association (Pajares et al., 2000; Pajares \& Valiante, 2001, but see Ryan et al., 2005, for a study documenting a negative association). Other researchers have concluded that the association between achievement goals and achievement changes as students grow older and move through the educational system (Bong, 2009; Midgley et al., 2001; Shim et al., 2008). Mastery goals were more adaptive in the elementary school years (Pekrun et al., 2009); that is, a positive relation to achievement outcomes, and became less adaptive at the secondary or college level, that is, a small positive or no relation to achievement outcomes. Conversely, performanceapproach goals were less adaptive in the early school years (e.g., Anderman \& Anderman, 1999) and became more adaptive at the secondary or college level (Harackiewicz et al., 1997). Middleton, Kaplan \& Midgley (2004) concluded that performance-approach goals may be less adaptive or potentially harmful for school achievement in elementary, middle, and high schools as the context is less competitive at those levels compared to the context of college (Middleton \& Midgley, 1997).

Bong (2009), in her study of 1,196 Korean students from grade one to grade nine, found no relation between achievement goals and achievement in grades one and two but found small positive correlations for mastery goals and small negative correlations for performance-avoidance goals for the higher grades. With respect to performance-approach goals, there was a small positive relation to achievement starting in the upper elementary school (grades five and six) and the middle school (grades seven to nine). There was no significant correlation for the lower grades. Thus, performance-approach goals did not play a significant role in the early school years. 
There are only a few German studies on the relation between achievement goals and school achievement. Köller (1998) found higher learning rates for mastery-goal oriented students than for performance-goal oriented students (grade seven). Paulick, Watermann and Nückles (2011c) and Finsterwald (2006) examined younger students ${ }^{\prime}$ achievement goals during the transition from elementary to secondary school. Paulick, Watermann \& Nückles 2011c) found (in an extension of the TIMS-study 2007) that students in grade four, $N=5,424$, demonstrated a negative association between performance-approach goals and school achievement and only a weak or no association between mastery/performance-avoidance goals and school achievement. Finsterwald (2006) also found no relation between achievement goals and school achievement during the transition. Thus, the research findings, until now, on the relation between achievement goals and school achievement during the transition to secondary school are inconsistent and not satisfying.

\subsection{The German school system}

As far as the transition from primary to secondary school is concerned, the German school system differs at least in two important ways from those in Anglo-Saxon countries. Firstly, in most German federal states, the transition to secondary school occurs after grade four. Secondly, after the transition, the children are separated according to their academic ability, the educational choices of their families, and the recommendations of their teachers. Most of the children are placed into one of three different school tracks: Hauptschule (low track), Realschule (middle track) or Gymnasium (academic track). These tracks are comparable in quantitative terms, such as the number of lessons timetabled, but differ markedly in qualitative terms. Specifically, there are differences among the tracks with respect to the compositional makeup, e.g., mean achievement and parental SES, and the institutional factors, such as lesson design, curriculum, and teacher training. On the one hand, the academic track provides qualitatively better conditions for academic learning because the learning group is positively selected in terms of social background and cognitive abilities (Trautwein, Lüdtke, Marsh et al., 2006) and the instruction is delivered at higher levels of cognitive activation (Kunter \& Baumert, 2006). On the other hand, as a consequence of ability-grouping, performance standards are higher in academic tracks than in non-academic tracks. To reach the performance level of the elementary school, students in the academic track have to exert greater effort and invest more than they did in elementary school. Thus, the learning environment of the academic track is more challenging as a result of a higher level of cognitive activation and 
a more demanding curriculum, and it is more competitive due to higher performance standards. Furthermore, social comparison might be more important compared to the learning environment of the non-academic track. The development of achievement goals during the transition and their role for student learning have been rarely studied taking these different learning environments into account.

\subsection{Research questions}

What do we know about the role of different learning environments for the development of achievement goals and their impact on learning? Maehr and Midgley (1991) suggested that performance goals increase more in learning environments that focus on grades and performance than in learning environments that focus on learning. As the academic track is more focused on grades and performance, one might expect an increase in performance goals and a decrease in mastery goals in these tracks. However, one could also expect an overall decline in all three types of achievement goals in both tracks (academic and nonacademic) because of a possible mismatch between the changing needs of the students and the changes in the secondary school learning environments.

Midgley et al. (2001) further suggested that the consequences of achievement goals may change as the context becomes more competitive. Mastery goals may become less adaptive (no relation to achievement), whereas performance goals may become more adaptive (positive relation to achievement). Therefore, Midgley et al. (2001) referred to a study by Harackiewicz et al. (1997) in which a positive relation between performance goals and the final grade in an introductory psychology class was found. They argued that college learning environments are often competitive and performance-oriented, which in turn leads to advantages of performance goals in these settings. Generally, the learning environment of secondary schools can also be described as more performance-oriented and, in some cases, as more competitive compared with the learning environment of elementary schools. Thus, we could expect that performance-approach goals might be slightly more adaptive in secondary schools than in elementary schools.

Accordingly, we expect differential effects of different school tracks on the impact of achievement goals on student learning.

Thus, our hypotheses and research questions are as follows:

1. How do achievement goals develop after the transition to secondary school? Can we find an overall decline in all three types of achievement goals as in other stu- 
dies that used the revised version of the PALS (Midgley et al., 2000)? Are there any differences between the school tracks?

2. How are achievement goals related to school achievement in elementary school? We expect a positive association between mastery goals and school achievement, whereas performance-approach goals might be less adaptive at this age and negatively associated with school achievement, the same as performance-avoidance goals.

3. How are achievement goals related to the development of school achievement in secondary school? We expect that mastery goals are still positively associated with school achievement after the transition to secondary school. However, do performance-approach goals become more adaptive? Do they lose their detrimental effect or do they predict school achievement positively?

4. As a result of the different learning environments, we assume that there could be differences between the predictive patterns of achievement goals in the different school tracks. Mastery goals should be more adaptive in the more challenging and cognitive activating academic track; that is, we expect a higher association between mastery goals and achievement in the academic track in comparison to nonacademic tracks.

\subsection{Method}

The empirical basis for the present investigation was the Transition Study, a cooperative project of the Max Planck Institute for Human Development in Berlin, the Department of Empirical Research in Education at the Freie Universität Berlin, the University of Potsdam, the Institute for School Development Research at the University of Dortmund, and the Institute for Educational Progress at the Humboldt University in Berlin. Germany's participation in the Trends in Mathematics and Science Study (TIMSS) 2007 of students in the fourth grade (T1) has provided an ideal opportunity to extend scientific knowledge of how students and their parents cope with the process of transition. As such, two further waves of data collection were undertaken after the transition to secondary school - six months later to coincide with the fifth grade mid-term report card [T2] and one year later to coincide with the final fifth grade report card [T3]. The Transition Study was embedded within TIMSS 2007. 


\subsubsection{Participants}

The sample of $N=1,646$ students in 246 schools (Gymnasium [academic track]: $n=$ 928, Realschule [middle track]: $n=436$, Hauptschule [low track]: $n=158$, other school types: $n=124)$ comprised most of the classes participating in the TIMSS. Due to the relatively small sample sizes of the middle and low tracks, we combined the tracks and the other school types into a single non-academic track $(n=718)$. We included students that participated in grade four and in at least one of the other two occasions. Of the sample, 49.4 percent were female, 87.4 percent of the children were born in Germany, and the mean age of the students at T1 was 10.4 years $(S D=0.50)$. Selectivity analyses revealed that the longitudinal sample was positively selected. Students whose parents selected the academic track (Gymnasium) were overrepresented in the longitudinal sample, while students with parents who selected the low track (Hauptschule) were underrepresented. Furthermore, students in the longitudinal sample had better classroom grades in grade four (approximately half of a standard deviation), and the socio-economic position of the parents was half of a standard deviation higher on average. In an additional step, a series of two-factor analyses of variance with the school track decision of the parents and the participation in the longitudinal sample as factors, as well as classroom grades, test scores and social-economic position of the parents as criteria, were conducted. No interaction effect was found, that is, school track decision $\times$ participation in the longitudinal sample, indicating that there was a systematic drop-out, but this drop-out did not differ systematically between students based on different school track decisions.

\subsubsection{Measures}

Achievement goals. Students were given surveys containing ten achievement goal items. Mastery-approach goal items (four items, $\alpha=.70$ ) stem from the revised version of the Patterns of Adaptive Learning Scales (PALS) (Midgley et al., 2000). The items focused on developing academic competence, for example, "It is important to me that I improve my skills", and were translated into German. According to Hulleman et al. (2010), we identified three components of mastery goals: mastering the requirements of the task (two items), improving one's competence, and fulfilling one's potential and learning to as great a degree as possible. We did not measure mastery-avoidance goals, and thus, mastery-approach goals are termed mastery goals, hereafter. Performance-approach goals were measured using four items $(\alpha=.83)$ based on the German version of the Motivational Orientation Scales (MOS) (Köller 
\& Baumert, 1998; original version by Nicholls et al., 1985). The items focused on demonstrating high academic competence relative to classmates, for example, "It is important to me that I know more than other students in my class". According to Hulleman et al. (2010), we identified two components of performance-approach goals: three normative items (trying to look good to others) and one appearance item (trying to do better than others). Performanceavoidance goals were assessed with two items $(\alpha=.59)$ that were based on items developed by Schwinger \& Wild (2006). The items addressed the focus on avoiding the demonstration of incompetence (appearance component). For example, "It is important to me that others do not think I am stupid”. All items were phrased generally and scored on a 4-point Likert-type scale $(1=$ very true through $4=$ not at all true). Items were reverse coded so that high scores on the final scales indicated high levels of achievement goals.

Achievement. Student classroom grades from grade four to grade five were used as a measure of performance achievement. We computed a GPA for three time points (middle of grades four and five, end of grade five) by taking the mean of four subjects (German, math, foreign language, social studies) in grade four and three subjects (German, math, foreign language) in grade five, respectively. The grades ranged from one to six, where one represented the best of all possible grades and six represented the worst. Grades were reverse coded so that higher scores on the final scales indicated higher levels of achievement.

Basic cognitive abilities. We also assessed the basic cognitive abilities of students using a German test of figural cognitive abilities (KFT; Heller \& Perleth, 2000) in two parallel versions (version A: Cronbach's $\alpha=.92$, version B: Cronbach's $\alpha=.93$ ). Students had to draw analogies between figures.

\subsubsection{Data analysis}

Analyses were conducted using the Mplus 6.1 program (Muthén \& Muthén, 19982010). Missing data were multiple imputed with Mplus. Multiple imputation is a procedure in which missing data are imputed based on the available information (Graham et al., 2003). Five complete data sets were created by filling in a random value for each missing data point. Mplus combines the results for each data set into an average result. Other treatments for missing data (e.g., listwise deletion, mean imputation) can lead to a biased statistical inference (Little \& Rubin, 2002). 
In the first step, the invariance of the measurement model (factor loadings) across groups was tested by means of a confirmatory factor analysis (CFA). All correlations between the factors were estimated freely.

To analyze the development of achievement goals, we used growth curve analytic techniques (Latent Growth Curve Models, LGCM; cf. Bollen \& Curran, 2006; Duncan, Duncan \& Strycker, 2006; Meredith \& Tisak, 1990) and compared the different models with each other. LGCM is a tool for analyzing longitudinal data that permits an analysis of change on a latent level whereby random measurement errors are considered. Thus, inter-individual differences of change and different forms of change over time can be taken into account. The slope factor indicates the rate of change per time unit. The interpretation of the slope factor depends on the presumed time function. In a linear growth curve model, a linear growth per time unit is expected. However, this time function is not adequate in every case. In some cases, it can be appropriate to estimate a model with an unspecified growth trend (Meredith \& Tisak, 1990) in which the time function is not fixed a priori but is estimated from the data. For each achievement goal, we specified different growth curve models, starting with a linear growth rate and followed by an unspecified (freed-loading LGCM) growth rate (Bollen \& Curran, 2006, Chap. 4). In the freed-loading LGCM, we fixed the first loading in the slope factor to 0 , the third loading to 1 , and freely estimated the remaining second loading. In addition, we imposed equality restrictions on the residuals of the repeated measures. To test whether coefficients differed across the different school tracks, we ran multi-group models for academic and non-academic tracks. To consider the nested structure of the data, we used the command type = general complex. The analyses were computed with the MLR estimator (maximum likelihood estimation with robust standard errors). As the value of the chi-squared statistic tends to increase along with the sample size, we considered approximate fit indices such as the comparative fit index (CFI), the Tucker-Lewis index (TLI), and the root mean square error of approximation index (RMSEA) for model evaluation. A very good fit is indicated by a value greater than 0.95 for the CFI and TLI and by a value less than 0.06 for the RMSEA (Hu \& Bentler, 1999; Yu, 2002).

To analyze the association between achievement goals and school achievement, we conducted cross-lagged panel models (Kenny, 1975). With regard to the time points T2 and T3, we used school-track centering for the GPA within the non-academic track to take into account the frame-of-reference effect on grading. Achievement goals were modeled as latent variables, constraining the factor loadings for each pair of repeated measures to be homogeneous over time. In addition, correlated errors have been specified for each pair of repeated 
measures (Marsh \& Yeung, 1997). The model consisted of 36 path coefficients relating the 12 constructs (nine achievement goals and three GPAs), whereby 15 of these paths were of critical importance: the six paths leading from each achievement goal to the subsequent GPA and the nine paths leading from the GPA to the subsequent achievement goal (see Figure 1 for a simplified model). To test whether path coefficients differ across the different school tracks, we ran multi-group models for academic and non-academic tracks. Five different cross-lagged panel models (CLPM) with different constraints were compared. All paths were estimated freely in model 1. In model 2, all autoregressive paths (stability coefficients between T1 and $\mathrm{T} 2$ and between $\mathrm{T} 1$ and $\mathrm{T} 3$ ) were constrained to be equal across academic and non-academic tracks. In model 3, all paths from previous GPAs to subsequent achievement goals, and in model 4, all paths from previous achievement goals to subsequent GPAs, were set equal across the different school tracks. In model 5, the paths from previous GPAs to subsequent achievement goals, and vice versa, were constrained to be equal across the school tracks. The model comparison was performed by using chi-square difference tests. In all models, we controlled for gender and cognitive ability. 


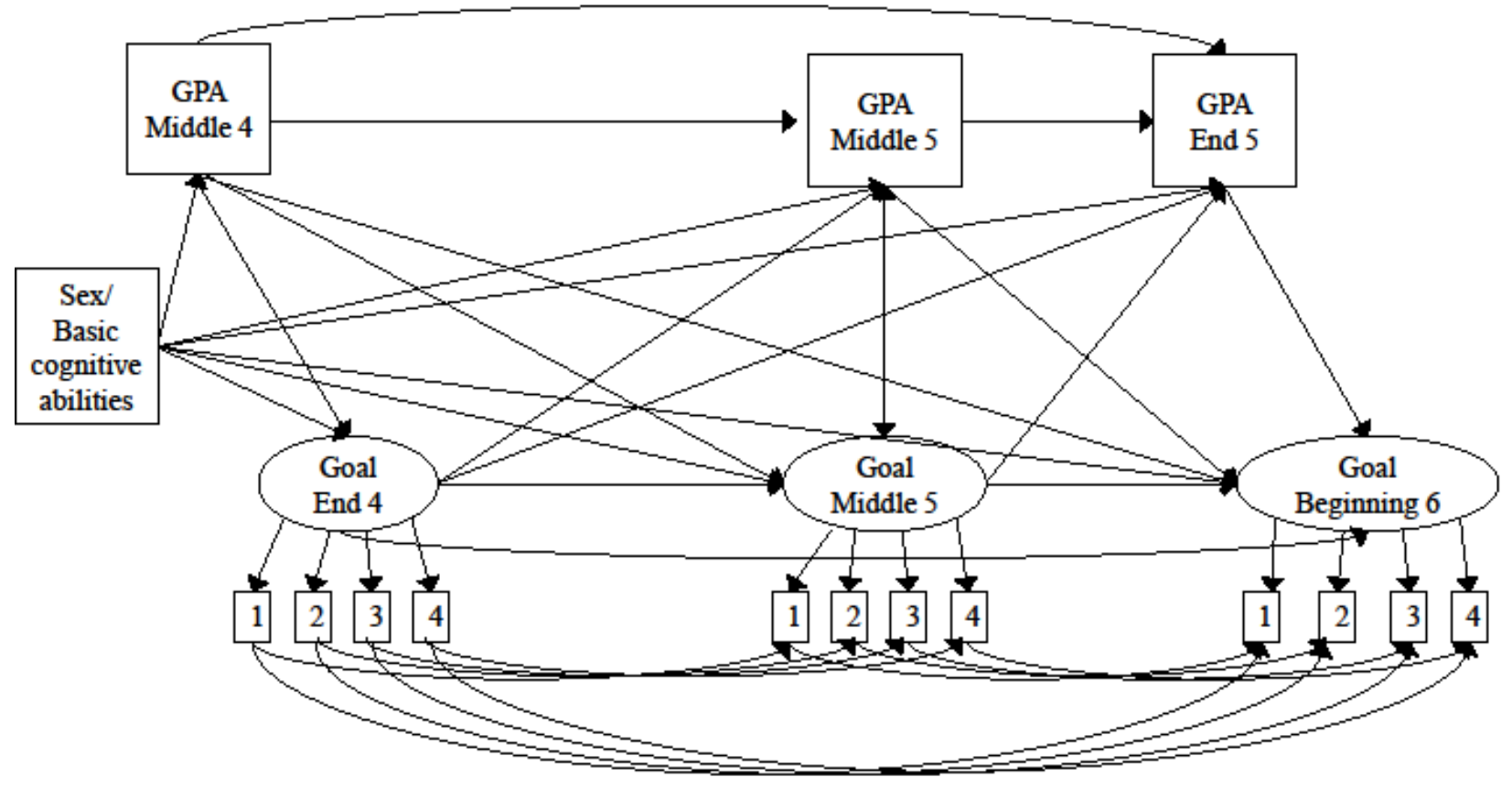

Figure 1. A priori model of causal paths between GPAs (GPA) and achievement goals (goal). For clarity, only a general achievement goal factor was pictured (goal) rather than all three types of achievement goals (mastery, performance-approach and performance-avoidance goals). Achievement goals were inferred from four indicators for mastery/performance-approach goals and two indicators from performance-avoidance goals. 


\subsection{Results}

The results of the CFAs revealed invariance of the measurement model across the groups. The model fit of a model with invariant factor loadings across groups was not significantly worse than a model with variant factor loadings (see Table 7).

\subsubsection{Descriptive statistics}

Table 5 presents means, standard deviations, and internal consistency reliabilities for the three achievement goal scales and the GPAs from T1 to T3 for the academic and nonacademic track. The mean level of all types of achievement goals declined from grade four to grade six in both tracks; thus, there were some differences in the mean levels across the tracks. Mastery goals differed only slightly across school tracks (Figure 2), whereas the mean levels of performance goals were higher for non-academic track students. We tested for the significance of the differences in the mean levels of the achievement goals between the school tracks by comparing two models. In model 1, we equated the means between the school tracks while in model 2, we freely estimated the means. Chi-square difference tests revealed significant mean level differences between the school tracks for mastery goals in grade four, which was slightly higher for the academic track (results of the $\chi^{2}$-difference tests: $X^{2}=6.55, d f=1$, $\mathrm{p}<.01)$. However, the tests did not reveal significant mean level differences for grades five and six (grade five: $X^{2}{ }_{\text {Diff }}=1.11, d f_{\text {Diff }}=1, \mathrm{p}>.05$; grade six: $X^{2}$ Diff $=0.52, d f_{\text {Diff }}=1, \mathrm{p}>$ $.05)$. With respect to the performance goals, the mean levels differed between the school tracks at all three time points (performance-approach goals grade four: $X^{2}$ Diff $=45.60, d f_{\text {Diff }}=$ $1, \mathrm{p}<.001$; grade five: $X^{2}$ Diff $=44.52, d f_{\text {Diff }}=1, \mathrm{p}<.001$; grade six: $X^{2}$ Diff $=36.86, d f_{\text {Diff }}=1$, $\mathrm{p}<.001$; performance-avoidance goals grade four: $X^{2}$ Diff $=20.19, d f_{\text {Diff }}=1, \mathrm{p}<.001$; grade five: $X^{2}$ Diff $=35.71, d f_{\text {Diff }}=1, \mathrm{p}<.001$; grade six: $X^{2}$ Diff $\left.=31.53, d f_{\text {Diff }}=1, \mathrm{p}<.001\right)$, thus indicating slightly lower means for students in the academic track.

As expected, as a result of the different grading practices, school grades declined in the academic track and increased in the non-academic track (see Figure 2). We tested for differences in the grades' mean levels between the school tracks. Chi-squared difference tests revealed significant mean level differences between the school tracks for GPAs in grade four, which were slightly better for the academic track (results of the $\chi^{2}$-difference tests: $X^{2}=$ 675.47, $d f=1, \mathrm{p}<.001)$, but again, there was not a significant difference in grades five and six (grade five: $X^{2}$ Diff $=1.78, d f_{\text {Diff }}=1, \mathrm{p}>.05$; grade six: $X^{2}{ }_{\text {Diff }}=2.64, d f_{\text {Diff }}=1, \mathrm{p}>.05$ ). 
Table 5

Descriptive statistics and reliabilities of achievement goals and GPAs in academic and nonacademic tracks from $T 1$ to $T 3$

\begin{tabular}{|c|c|c|c|c|c|c|}
\hline \multirow[b]{2}{*}{ Scales } & \multicolumn{3}{|c|}{ Academic Track } & \multicolumn{3}{|c|}{ Non-academic Track } \\
\hline & $M$ & $S D$ & $\alpha$ & $M$ & $S D$ & $\alpha$ \\
\hline MAP $4^{\text {th }}$ grade & 3.57 & 0.45 & .74 & 3.51 & 0.49 & .78 \\
\hline MAP $5^{\text {th }}$ grade & 3.46 & 0.46 & .73 & 3.44 & 0.47 & .74 \\
\hline MAP $6^{\text {th }}$ grade & 3.21 & 0.48 & .73 & 3.21 & 0.49 & .73 \\
\hline PAP $4^{\text {th }}$ grade & 2.42 & 0.87 & .84 & 2.72 & 0.82 & .88 \\
\hline PAP $5^{\text {th }}$ grade & 1.88 & 0.74 & .87 & 2.14 & 0.79 & .86 \\
\hline PAP $6^{\text {th }}$ grade & 1.71 & 0.65 & .67 & 1.92 & 0.75 & .88 \\
\hline PAV $4^{\text {th }}$ grade & 2.93 & 0.65 & .77 & 3.08 & 0.66 & .62 \\
\hline PAV $5^{\text {th }}$ grade & 2.50 & 0.76 & .74 & 2.70 & 0.65 & .75 \\
\hline PAV $6^{\text {th }}$ grade & 2.14 & 0.88 & .84 & 2.36 & 0.74 & .85 \\
\hline GPA Middle $4^{\text {th }}$ grade & 4.12 & 0.45 & .68 & 3.20 & 0.63 & .58 \\
\hline GPA Middle $5^{\text {th }}$ grade & 3.61 & 0.68 & .75 & 3.29 & 0.61 & .62 \\
\hline GPA End $5^{\text {th }}$ grade & 3.56 & 0.67 & .75 & 3.28 & 0.61 & .61 \\
\hline
\end{tabular}

Note $. \mathrm{M}=$ Mean. $\mathrm{SD}=$ Standard deviation. $\mathrm{MAP}=$ Mastery goal. PAP $=$ Performanceapproach goal. PAV = Performance-avoidance goal. 
Mastery goals

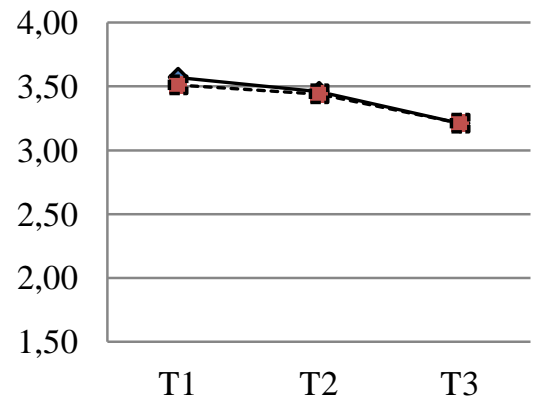

Performance-avoidance goals

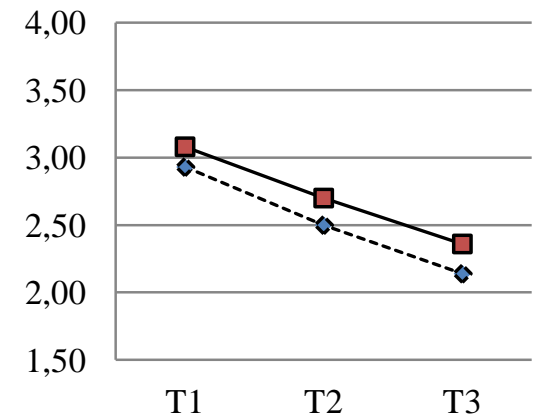

Performance-approach goals

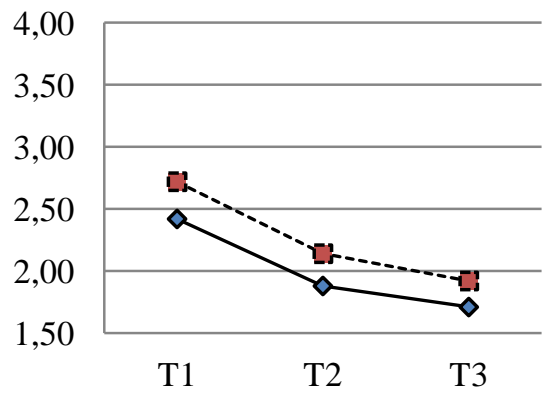

GPA

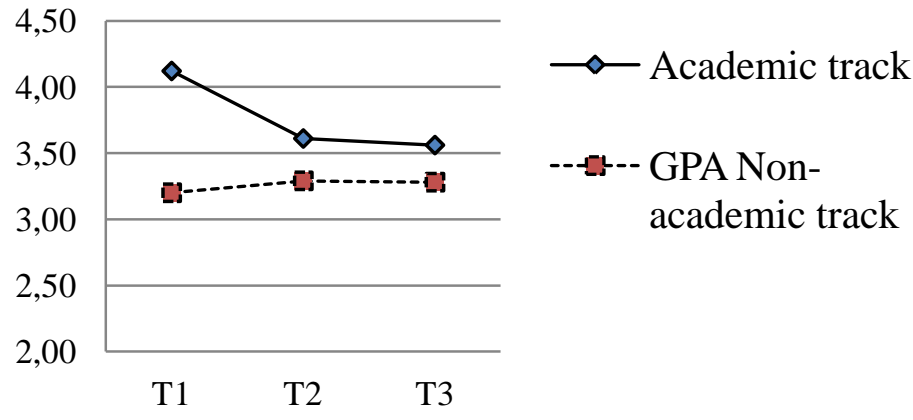

Figure 2. Means of achievement goals and GPA from grades four to six for academic and non-academic tracks. $\mathrm{T} 1=$ End of grade four. $\mathrm{T} 2=$ Middle of grade five. $\mathrm{T} 3=$ Beginning of grade six.

Table 6 presents correlation coefficients among the variables within each school track. Several findings are noteworthy. First, the positive correlation between mastery and performance goals decreased after the transition in both school tracks. Second, there were no or only minimal correlations between achievement goals and GPAs. While the non-academic track student mastery goals showed no significant correlation to GPAs, there was a significant correlation for academic track students in grade six. Performance-approach and performanceavoidance goals showed small negative correlations with GPAs in both tracks. The correlations between performance-approach goals in grade four and GPAs were significant in both school tracks, whereas later correlations were significant only in non-academic tracks. Only performance-avoidance goals in grade four were significantly related to GPAs in nonacademic tracks, whereas in academic tracks, performance-approach goals in grade five were also significantly correlated to GPAs. 
Table 6

Pearson-correlation coefficients of measures

\begin{tabular}{|c|c|c|c|c|c|c|c|c|c|c|c|c|}
\hline Measure & 1 & 2 & 3 & 4 & 5 & 6 & 7 & 8 & 9 & 10 & 11 & 12 \\
\hline 1. MAP $4^{\text {th }}$ grade & - & .37 & .25 & .37 & .16 & .09 & .40 & .18 & .09 & -.02 & .02 & .03 \\
\hline 2. MAP $5^{\text {th }}$ grade & .34 & - & .49 & .18 & .17 & .10 & .16 & .22 & .06 & .01 & .05 & .09 \\
\hline 3. MAP $6^{\text {th }}$ grade & .25 & .29 & - & .10 & .15 & .14 & .10 & .15 & .11 & .08 & .10 & .11 \\
\hline 4. PAP $4^{\text {th }}$ grade & .39 & .22 & .13 & - & .51 & .40 & .81 & .45 & .37 & -.17 & -.18 & -.15 \\
\hline 5. PAP $5^{\text {th }}$ grade & .11 & .24 & .17 & .37 & - & .61 & .42 & .76 & .51 & -.06 & -.09 & -.07 \\
\hline 6. PAP $6^{\text {th }}$ grade & -.00 & .11 & .22 & .32 & .55 & - & .32 & .45 & .76 & -.07 & -.03 & -.02 \\
\hline 7. PAV $4^{\text {th }}$ grade & .42 & .21 & .09 & .81 & .31 & .22 & - & .44 & .34 & -.11 & -.13 & -.11 \\
\hline 8. PAV $5^{\text {th }}$ grade & .10 & .30 & .16 & .20 & .76 & .43 & .27 & - & .47 & -.09 & -.11 & -.11 \\
\hline 9. $\mathrm{PAV} 6^{\text {th }}$ grade & .04 & .14 & .26 & .29 & .50 & .78 & .24 & .46 & - & -.07 & -.06 & -.05 \\
\hline 10. GPA Middle $4^{\text {th }}$ grade & .08 & .02 & .03 & -.16 & -.14 & -.11 & -.06 & -.05 & -.11 & - & .51 & .52 \\
\hline 11. GPA Middle $5^{\text {th }}$ grade & .06 & .03 & .00 & -.11 & -.08 & -.12 & -.03 & -.05 & -.12 & .38 & - & .85 \\
\hline 12. GPA End $5^{\text {th }}$ grade & .07 & .09 & .07 & -.09 & -.10 & -.13 & -.02 & -.06 & -.12 & .40 & .76 & - \\
\hline
\end{tabular}

Note. Academic track students $(n=928)$ are shown above the diagonal. Non-academic track students $(n=718)$ are shown below the diagonal. MAP = Mastery goal. PAP = Performance-approach goal. PAV = Performance-avoidance goal. Coefficients greater than .09 in absolute value for academic track and .11 for non-academic track are statistically significant at $p<.01$. 


\subsubsection{Growth curve models}

To test the development of achievement goals and GPA, we used growth curve analytic techniques and compared growth curve models with two different time functions, that is, linear vs. unspecified. For all three types of achievement goals and for GPAs, a model with an unspecified time function showed a significantly better model fit than a model with a linear time function (results of the $\chi^{2}$-difference tests: mastery goals: $X^{2}$ Diff $=45.17, d f_{\text {Diff }}=1, \mathrm{p}<$ .001 ; performance-approach goals: $X^{2}{ }_{\text {Diff }}=115.19, d f$ Diff $=1, \mathrm{p}<.001$; performanceavoidance goals: $X^{2}$ Diff $=6.73, d f_{\text {Diff }}=1, \mathrm{p}<.001$; GPA: $X^{2}$ Diff $\left.=78.34, d f_{\text {Diff }}=1, \mathrm{p}<.001\right)$. The slope factor of mastery goals (-.278) indicated that 27.8 percent of the change occurred during the first half of the school year in secondary school. With respect to performance goals, the percentage of change during the first half of the school year in grade five was much higher at 73.3 percent for performance-approach goals and 55.5 percent for performanceavoidance goals. The results for student GPAs showed a change of 90.2 percent between T1 and $\mathrm{T} 2$.

In an additional step, we tested for differences in the trajectories (factor loadings, residual variances) and covariances between the intercept and the slope factor of the growth curve models across school tracks. For mastery, performance-approach goals and GPAs, a model with equated factor loadings and residual variances across the school tracks but with differing residual variances of the repeated measures showed the best model fit (mastery goals: $X^{2}=$ 5.09, $d f=4, p>$.05., RMSEA $=.016, \mathrm{CFI}=.998, \mathrm{TLI}=.997$; performance-approach goals: $X^{2}=18.97, d f=4, \mathrm{p}<.001, \mathrm{RMSEA}=.067, \mathrm{CFI}=.984, \mathrm{TLI}=.976 ; \mathrm{GPA}: X^{2}=14.84, d f=4$, $\mathrm{p}<.01, \mathrm{RMSEA}=.057, \mathrm{CFI}=.994, \mathrm{TLI}=991)$. For performance-avoidance goals, a model with equated trajectories and residual variances of the repeated measures and across the groups fit the data best $\left(X^{2}=8.71 d f=6, \mathrm{p}>.05, \mathrm{RMSEA}=.022, \mathrm{CFI}=.996, \mathrm{TLI}=.996\right)$.

\subsubsection{Cross-lagged panel models}

The results of the cross-lagged panel models and the model comparisons revealed that the model fit worsened when equality restrictions across groups were imposed on the autoregressive coefficients as well as on the coefficients from previous achievement goals to subsequent GPAs (see Table 7). Therefore, in an additional step, we tested for differences across the academic and the non-academic track by estimating paths freely, one at a time. A model where all but four paths were constrained to be equal across the school tracks fit the data best: 
the path between performance-approach goals in grade four and GPA in the middle of grade five and the paths between each GPA differed significantly across academic and nonacademic tracks (GPA middle 4 to GPA middle 5: academic track $B=.67, p<.001$, nonacademic track $B=.39, p<.001$; GPA middle 5 to GPA end 5: academic track $B=.77, p<$ .001 , non-academic track $B=.68, p<.001)$. The freely estimated paths between each GPA showed that the GPAs were less stable in non-academic tracks than in academic tracks. 
Table 7

Test of model fit and chi-square difference test for the cross-lagged panel models

\begin{tabular}{|c|c|c|c|c|c|c|c|c|c|c|}
\hline \multirow[t]{2}{*}{ Model } & \multicolumn{4}{|c|}{ Equality constraints across groups } & \multicolumn{6}{|c|}{ Fit indices } \\
\hline & FL & AR & GPA ON AG & AG ON GPA & $\mathrm{X}^{2}$ & df & $\mathrm{p}$ & CFI & TLI & RMSEA \\
\hline & \multicolumn{10}{|c|}{ Confirmatory Factor Analysis (CFA) } \\
\hline M1 & & & & & 1804,16 & 727 & .000 & .946 & .936 & .042 \\
\hline M2 & $\mathrm{x}$ & & & & 1814,44 & 734 & .000 & .946 & .936 & .042 \\
\hline$\Delta \mathrm{M} 2-\mathrm{M} 1$ & & & & & 10,27 & 7 & .174 & & & \\
\hline & Cro & $\overline{\text { agged }}$ & el Model (CLP & & & & & & & \\
\hline M1 & $\mathrm{x}$ & & & & 2544,96 & 980 & .000 & .933 & .919 & .044 \\
\hline M2 & $\mathrm{x}$ & $\mathrm{x}$ & & & 2586,71 & 992 & .000 & .932 & .918 & .044 \\
\hline M3 & $\mathrm{x}$ & $\mathrm{x}$ & $\mathrm{x}$ & & 2604,01 & 1001 & .000 & .931 & .918 & .044 \\
\hline M4 & $\mathrm{x}$ & $\mathrm{x}$ & & $\mathrm{x}$ & 2604,55 & 1007 & .000 & .931 & .918 & .044 \\
\hline M5 & $\mathrm{x}$ & $\mathrm{x}$ & $\mathrm{x}$ & $\mathrm{x}$ & 2621,59 & 1016 & .000 & .930 & .920 & .044 \\
\hline$\Delta \mathrm{M} 2-\mathrm{M} 1$ & & & & & 41,75 & 12,00 & .000 & & & \\
\hline$\Delta \mathrm{M} 3-\mathrm{M} 2$ & & & & & 17,30 & 9,00 & .044 & & & \\
\hline$\Delta \mathrm{M} 4-\mathrm{M} 2$ & & & & & 13,03 & 8,00 & .111 & & & \\
\hline$\Delta \mathrm{M} 5-\mathrm{M} 2$ & & & & & 34,88 & 24,00 & .070 & & & \\
\hline$\Delta \mathrm{M} 5-\mathrm{M} 1$ & & & & & 76,63 & 36,00 & .000 & & & \\
\hline M6 & $\mathrm{x}$ & $\mathrm{x} 1$ & $\mathrm{x} 2$ & $X$ & 2583,57 & 1012 & .000 & .932 & .921 & .043 \\
\hline$\Delta \mathrm{M} 6-\mathrm{M} 1$ & & & & & 38,61 & 32,00 & .195 & & & \\
\hline
\end{tabular}

Note. $\mathrm{FL}=$ Factor loadings, $\mathrm{AR}=$ Autoregressive coefficients, $\mathrm{GPA}=$ Grade Point Average, $\mathrm{AG}=$ Achievement goals, $\mathrm{X}^{2}=\mathrm{Chi}-\mathrm{square}, \mathrm{df}=\mathrm{de}-$ grees of freedom, 1 = free estimation of autoregressive coefficients between grades, 2 = free estimation of GPA (T2) on PAP (T1). 
Table 8 shows the results of the final CLPM while always controlling for the other achievement goals, gender, and basic cognitive abilities. GPA in the middle of grade four predicted mastery goals positively, and performance-approach goals were predicted negatively at the end of grade four. Performance-approach goals in grade four negatively predicted achievement after the transition to the academic track in secondary school. Furthermore, mastery goals predicted later higher school achievement, both in the academic and the nonacademic tracks (cross-lagged effects). Performance-avoidance goals did not predict achievement at any time point or for any school track.

The remaining path coefficients, those relating nonadjacent constructs (e.g., GPA in the middle of grade 4 to achievement goal at the beginning of grade 6), were insignificant. 
Table 8

Unstandardized and standardized path coefficients for the final two-group cross-lagged panel model

\begin{tabular}{|c|c|c|c|}
\hline Paths & & $\begin{array}{l}\text { Unstandardized } \\
\text { estimates }\end{array}$ & $\begin{array}{l}\text { Standardized } \\
\text { estimates }\end{array}$ \\
\hline \multicolumn{4}{|l|}{ Stability } \\
\hline MAP End 4 & $\rightarrow$ MAP Middle 5 & $.43 * * *$ & $.45^{* * *}$ \\
\hline MAP Middle & $\rightarrow$ MAP Beginning 6 & $.59 * * *$ & $.57 * * *$ \\
\hline PAP End 4 & $\rightarrow$ PAP Middle 5 & $.39 * * *$ & $.41 * * *$ \\
\hline PAP Middle 5 & $\rightarrow$ PAP Beginning 6 & $.50 * * *$ & $.55^{* * *}$ \\
\hline PAV End 4 & $\rightarrow$ PAV Middle 5 & $.48 * * *$ & $.36 * * *$ \\
\hline PAV Middle 5 & $\rightarrow$ PAV Beginning 6 & $.56 * * *$ & $.42 * * *$ \\
\hline GPA Middle 4 & $\rightarrow$ GPA Middle 5 & $.67 * * * / .39 * * *$ & $.45 * * * / .37 * * *$ \\
\hline GPA Middle 5 & $\rightarrow$ GPA End 5 & $.77 * * * / .68 * * *$ & $.77 * * * / .70 * * *$ \\
\hline \multicolumn{4}{|c|}{ From achievement goal to GPA } \\
\hline MAP End 4 & $\rightarrow$ GPA Middle 5 & $.14^{*}$ & $.09 *$ \\
\hline PAP End 4 & $\rightarrow$ GPA Middle 5 & $-.10 * * /-.03$ & $-.12 * * /-.04$ \\
\hline PAV End 4 & $\rightarrow$ GPA Middle 5 & .02 & .02 \\
\hline MAP Middle & $\rightarrow$ GPA End 5 & $.12 * * *$ & $.07 * * *$ \\
\hline PAP Middle 5 & $\rightarrow$ GPA End 5 & -.02 & -.02 \\
\hline PAV Middle 5 & $\rightarrow$ GPA End 5 & -.02 & -.02 \\
\hline \multicolumn{4}{|c|}{ From GPA to achievement goal } \\
\hline GPA Middle 4 & $\rightarrow$ MAP End 4 & $.06^{*}$ & $.09 *$ \\
\hline GPA Middle 4 & $\rightarrow$ PAP End 4 & $-.15 * * *$ & $-.11 * * *$ \\
\hline GPA Middle 4 & $\rightarrow$ PAV End 4 & .01 & .01 \\
\hline GPA Middle 5 & $\rightarrow$ MAP Beginning 6 & -.03 & -.04 \\
\hline GPA Middle 5 & $\rightarrow$ PAP Beginning 6 & -.03 & -.03 \\
\hline GPA Middle 5 & $\rightarrow$ PAV Beginning 6 & -.05 & -.04 \\
\hline GPA End 5 & $\rightarrow$ MAP Beginning 6 & .03 & .05 \\
\hline GPA End 5 & $\rightarrow$ PAP Beginning 6 & .03 & .03 \\
\hline GPA End 5 & $\rightarrow$ PAV Beginning 6 & .00 & .00 \\
\hline
\end{tabular}

Note. $\mathrm{MAP}=$ Mastery goal. $\mathrm{PAP}=$ Performance-approach goal. $\mathrm{PAV}=$ Performanceavoidance goal. The paths between the GPAs and the path from PAP End 4 to GPA Middle 5 differed between the academic and non-academic track. The coefficient before the backslash is for the academic track and behind the slash for the non-academic track.

$* p<.05 . * * p<.01 . * * * p<.001$ 


\subsection{Discussion}

Our main goals in this study were to examine the development of achievement goals and the relations between achievement goals and school achievement in two different school tracks (academic vs. non-academic) during the transition from primary to secondary school. Our central research questions and hypotheses were as follows. Can we find an overall decline in all three types of achievement goals? Are there any differences between the school tracks? How are the achievement goals related to school achievement before and after the transition? We expected a positive relation between mastery goals and achievement before and after the transition. For performance goals, a negative association with school achievement was expected in elementary school. Do performance-approach goals become adaptive in secondary schools? Do they predict achievement positively after the transition? Are there any differences between the predictive patterns of achievement goals in the different school tracks?

The results revealed an overall decline in all three types of achievement goals from grades four to six in each school track. There were only slight differences between the school tracks in our study. High-achieving students that moved to an academic track were slightly less performance-goal oriented at all three time points and slightly more mastery-goal oriented at the end of grade four than students who moved to a non-academic track. This result supports the findings of Köller (1998) and Finsterwald (2006). Köller (1998) showed that students in academic tracks were less performance-goal oriented than students in nonacademic tracks, even though he studied seventh graders. Similarly, Finsterwald (2006) proved that performance goals were less salient for academic track students than for middle track students (Realschule) in grade five.

Interestingly, performance goals, especially performance-approach goals, declined much more rapidly than mastery goals $(27.8 \%)$. During the first half of the school year in secondary school, three-fourths of the decline in performance-approach and more than half of the decline in performance-avoidance goals occurred. The transition to secondary school and the changes that come along with that transition seem to have impacted the development of achievement goals, especially performance goals. Performance goals are characterized as more achievement related and susceptible to social comparison processes than mastery goals. Because students's school achievement worsened after the transition to the academic track in secondary school, one could argue that performance goals were especially vulnerable to these changes what resulted in a much faster decline in these type of achievement goal. In contrast, 
for mastery goals intra-individual comparisons are more important, which might have been less influenced by the achievement-related changes than performance goals. Indeed, studies on the impact of the transition to secondary school on different motivational variables have shown that the effect of the transition on students's self-concept occurs faster than on students' intrinsic motivation (e.g., Paulick, Watermann \& Nückles, in preparation).

The results of the LGCMs indicated that a model with equated factor loadings fit the data best for all three types of achievement goals. Thus, the development of achievement goals and the changes that are associated with the transition appear similar in both school tracks. This result suggests that the institutional effect on the development of achievement goals seems to be comparable and independent of the school track. Our results could be an indicator for the assumption that the secondary school could be a source of decline in achievement goals or in motivation more generally during early adolescence (Anderman \& Anderman, 1999; Anderman \& Midgley, 1997). It is conceivable that the students' intrapersonal changes that are associated with the onset of pubertal changes have resulted in the overall decline of achievement goals. The overall decline in all three types of achievement goals could be interpreted as evidence of a mismatch between the needs of early adolescents and the classroom environment of secondary schools (stage-environment fit, Eccles \& Midgley, 1989). This mismatch could be observed in both the academic track and the non-academic track. However, we cannot clarify what specific changes in the classroom environment influenced the development of achievement goals.

Our findings for the development of achievement goals are consistent with the results of other studies that used the revised version of the PALS (Bong, 2009; Finsterwald, 2006; Shim et al., 2008) and also reveal a general decline of all three types of achievement goals. Thus, to ensure that our results are specific for the measurement instrument used, a replication of our findings with another instrument would be necessary and should be conducted in a future study.

Our second research question referred to the relation between achievement goals and school achievement in elementary school. The hypothesis that mastery goals are positively associated with school achievement can be confirmed. The results of the cross-lagged panel models proved that there was a positive relation between mastery goals and school achievement in elementary school. The more mastery-goal oriented the students were in the middle of grade four, the better their grades were at the end of grade four. This positive effect of mastery goals remained significant after the transition to secondary school and was the same in academic and non-academic tracks. The hypothesis that mastery goals are more adaptive in 
the academic tracks could not be confirmed. Mastery goals were related to school achievement independent of the school track. The more cognitively activated learning environment in the academic track did not lead to a higher relation between mastery goals and school achievement.

As expected, performance-approach goals were negatively related to school achievement in elementary school. This type of achievement goal has been proven to be disadvantageous for the achievement of young students. In fact, after the transition to secondary school, low achievement was predicted by performance-approach goals even if only at the academic track. The more performance-approach goal oriented the students were at the end of elementary school, the worse their GPAs later on.

Contrary to our expectations, performance-approach goals did not become more adaptive after the transition to secondary school. This type of achievement goal was still negatively related to academic achievement in the track that we characterized as more competitive the academic track. As suggested by Middleton et al. (2004), we found that this performanceapproach goal had a negative effect on school achievement as early as elementary school or vice versa, that is, school achievement potentially negatively influenced the adoption of performance-approach goals. The direction of this relation is unclear as we assessed the variables shortly after another. We can confirm, however, that performance-approach goals are not adaptive for young students (Midgley et al., 2001). Rather, they are related to lower achievement in learning environments with a high level of cognitive activation and high performance standards.

A possible explanation for the negative effect of performance-approach goals on school achievement could be the use of certain learning strategies. As explained above, performance goals have often been shown to be related to the use of surface-learning strategies (e.g., Ames \& Archer, 1988; Bouffard et al., 1995; Meece et al., 1988; Midgley \& Urdan, 2001; Nolen, 1988; Köller, 1998; Pintrich \& De Groot, 1990). The use of these learning strategies could be especially disadvantageous in learning environments that are highly challenging: a more demanding curriculum or a higher level of cognitive activation during lessons, as is often the case in the academic track. Therefore, the use of deep-processing learning strategies could be more important in academic tracks than in non-academic tracks. Accordingly, a high performance-approach goal orientation could lead to lower school achievement, and vice versa, in highly challenging learning environments mediated by the use of surface-learning strategies. Another possible mediating variable between performance-approach goals and school achievement could be emotions. Negative emotions such as anxiety have been found to 
be related to low achievement (e.g., Pekrun, Goetz, Titz \& Perry, 2002) and to performance goals. Thus, one could argue that the association between performance-approach goals and low achievement could be mediated by negative emotions.

Moreover, our study has shown that grades did not predict achievement goals, which is an interesting finding because some researchers have identified perceived competence as a probable precursor to goal adoption (Elliot \& Church, 1997). It was argued that students with high perceived competence would be likely to endorse approach goals, whereas students with low perceived competence would be likely to adopt avoidance goals. As grades are known to be the most important source for a student's perceived competence, our results do not support this argument with respect to the transition from primary to secondary school.

The effect sizes for achievement goals and school achievement were relatively small. However, we consider the prediction pattern as significant and relevant in view of the fact that the other dimensions of achievement goals, gender and cognitive ability, were controlled at all three time points. Under these conditions, even very small effects should be interpreted as meaningful.

There are a few limitations worthy of consideration. First, we measured achievement goals only as domain-general, not as domain-specific, separately for each school subject. Current research is not clear on this issue. Some researchers are convinced that achievement goals are domain-general (e.g., Köller \& Baumert, 1998; Nicholls, 1992), while others think achievement goals are domain-specific (Middleton \& Midgley, 1997; Miller et al., 1993; Wigfield \& Eccles, 1992; Wigfield et al., 1991). Recent studies differentiated between mastery and performance goals and proved that performance goals are domain-general and mastery goals are domain-specific (Bong, 2001; Sparfeldt, Buch, Wirthwein \& Rost, 2007). Because we did not assess achievement domain-specific goals, we cannot be certain that achievement goals do not differ among single school subjects.

Second, we only assessed grades as indicators of achievement. However, grades are considered less objective than standardized tests. Furthermore, there are differences in teachers' judgments about student academic achievement (grading) between the school tracks that should not be neglected. The reference group plays an important role when students are graded (Südkamp \& Möller, 2009), whereas standardized tests provide more objective information about student achievement. Of great interest would be a replication of our studies using standardized tests instead of grades.

As our study consisted only of three time points, we can draw conclusions only with caution. The results seem to support the assumptions of Midgley et al. (2001) and Grant and 
Dweck (2003) in that the consequences of achievement goals change as students move through the educational system. Thus, our results seem to suggest that the school track does not have a differential impact on the development of achievement goals and only a small impact on the association between achievement goals and achievement. Before drawing conclusions for implications for practice, additional time points should be included.

As Hulleman et al. (2010) showed, the association between achievement goals and achievement depends considerably on the measuring instrument used and the construct behind it. Further research should consider a more in-depth measuring instrument used because it cannot be concluded that the results are independent of the used construct.

Our results extend previous findings that investigated the development of achievement goals and their relation to school achievement because we included different school tracks in our study. We confirmed an overall decline of motivation or, rather, achievement goals during the transition from elementary to secondary school. Furthermore, the results demonstrate that prior mastery goals have significant effects on subsequent school achievement beyond the effects of prior achievement (gender and cognitive abilities), independent of the school track. Our study also demonstrates that performance-approach goals are detrimental for school achievement of young students (elementary school, academic track of secondary school). Future research should identify the characteristics of the learning environment or the reference group that play an important role in the development of achievement goals and their relation to school achievement so as to specifically counteract the decline in motivation that occurs during the transition to secondary school. Thus, the learning environment can be structured in a way that arouses mastery goals, thus supporting the positive effect of this type of achievement goal on student school achievement. 


\section{Effects of tracking on students' self-concept, intrinsic motivation, and achievement goals during the transition to secondary school (Studie 4$)^{11}$}

\subsection{Abstract}

Tracking, or ability grouping, has proved to be a widespread educational practice with far-reaching consequences. Going beyond the focus of prior studies on frame-of-reference effects, the present research examined the effect of between-school tracking not only on students' self-concept and intrinsic motivation but also on students' achievement goals during the transition from elementary school to different school tracks (academic vs. non-academic tracks) in secondary school. Conditional latent growth curve models were conducted using data from a large-scale three-wave assessment examining 1,646 students in grades four to six. Results indicated that, given comparable individual achievements, students's self-concept, intrinsic motivation, and mastery goals decreased significantly more strongly when the students transitioned to academic compared to non-academic tracks. Classroom grades partially mediated the contrast effect on students's self-concept and mastery goals. No frame of reference effect was found for performance goals.

\subsection{Introduction}

Tracking, or ability grouping, is a widespread form of achievement grouping used by many educational systems around the world. A significant body of research has addressed how tracking influences different psychological constructs, such as self-concept, self-esteem, and interest as well as students' achievement (e.g., Chiu et al., 2008; Marsh et al., 2001; Marsh et al., 2000; Trautwein, Lüdtke, Marsh, et al., 2006). Remarkably, there is no known research on the effect of tracking on achievement goals, which are considered a part of motivation (i.e., part of self-motivational beliefs of self-regulation, Zimmerman, 1998). Achievement goals are also related to students' learning in school (see meta-analysis by Hulleman et al., 2010). The present study is designed to correct this omission by investigating the effect of between-school tracking on students's self-concept and intrinsic motivation and on achievement goals during the transition to different school tracks in secondary school.

\footnotetext{
${ }^{11}$ Dieser Abschnitt basiert auf einem in Vorbereitung befindlichen Manuskript (Paulick, Watermann \& Nückles, 2011b).
} 
The transition to secondary school is a source of decline in students' motivation (e.g., Anderman \& Maehr, 1994; Eccles \& Midgley, 1989). Studies on the stage-environment fit theory (Eccles et al., 1997; Juvonen et al., 2004) revealed that changes in classroom variables contributed to the decline in students' motivation. The relevant classroom variables included organizational, instructional, and climate variables. As students grow older, their needs change. The desire for autonomy and self-determination, peer orientation or identity issues become more important. In light of these new needs, the environmental changes that are often associated with the transition to secondary school (e.g., emphasis on competition, social comparison) seem especially harmful. These changes could result in a developmental mismatch between an early adolescent's needs and the classroom environment, and this mismatch may cause a decline in motivational outcomes (Eccles et al., 1993).

\subsection{Aspects of tracking}

Other researchers noted that tracking can have a negative effect on different outcome variables (e.g., Marsh \& Hau, 2003; Trautwein et al., 2005). Streaming, tracking or ability grouping students within an educational system is a widespread phenomenon around the world that is highly controversial among experts. Experts opposed to ability grouping argue that it prevents equality of educational opportunities to many students and limits their life chances. Experts who support ability grouping argue that it allows more effective teaching that is adapted to the ability level of the specific group or track (see Ireson \& Hallam, 2001). Research has shown that ability grouping impacts multiple outcomes, including students' achievement, self-concept, self-esteem, and interest.

Different forms of tracking that should be considered: tracking within or between schools, school types, and classrooms or courses. The present study refers to tracking between schools because this type of tracking is particularly common in Germany. Other countries that use explicit tracking to different school types included the Netherlands and Belgium. In this form of tracking, the prior achievement of students is the most important factor in assigning students to different school tracks, but academic specializations also matter. 


\subsection{Effects of tracking}

Most research on effects of tracking has focused on outcome variables such as students's self-concept (academic or domain-specific), interest or self-esteem. Researchers have found that tracking can have a negative effect on these variables (e.g., Trautwein et al., 2005). The so-called big-fish-little-pond effect (BFLPE; e.g., Marsh, 1987) is a contrast effect that causes a weakening in academic self-concept among students in high-achievement groups. The average achievement level of the track negatively affects students' self-concept. The main reasons for this contrast effect are social comparisons among students (frame-ofreference effects; Marsh, 1986). Students compare their own achievements with the achievements of their schoolmates or classmates and integrate the results of this comparison into their self-concept. In addition to students' self-concept, other studies have identified the BFLPE in students' educational aspirations (Marsh \& O’Mara, 2010) and interest (e.g., Köller, Trautwein, Lüdtke \& Baumert, 2006; Trautwein, Lüdtke, Marsh et al., 2006). In the latter studies, the BFLPE on students' interest was mediated by students' self-concept (Köller, Schnabel \& Baumert, 2000; Trautwein, Lüdtke, Marsh et al., 2006). Thus, students's self-concept appears to be a key variable in educational settings (Marsh, Trautwein, Lüdtke, Köller \& Baumert., 2005).

\subsection{The present investigation}

The present study focused on tracking effects during the transition to different secondary school tracks in Germany. The German school system differs in many ways from the school systems in Anglo-Saxon countries. In most German federal states, the transition from primary to secondary school occurs immediately after completion of grade four, when students are approximately 10 years of age. Students were segregated into groups and transitioned to different school types according to their academic abilities, the educational choices of their families, and the recommendations of their teachers. Most of the children attend one of three different school types: Hauptschule (low track), Realschule (middle track) or Gymnasium (academic track). Academic demand is lowest in the Hauptschule track, intermediate in the Realschule track, and highest in the Gymnasium track. The German "tripartite" system can be described as a form of school-type tracking based on students' achievement differences, and the resultant track has implications for the students' future careers (Trautwein, Lüdtke, Marsh et al., 2006). The different tracks are comparable in quantitative terms (i.e., number of 
lessons timetabled) but differ markedly in qualitative terms. Specifically, the characteristics of the tracks differ in both composition (e.g., mean achievement, parental SES) and institution (e.g., lesson design, curriculum, teacher training). The learning group in the academic track is positively selected in terms of social background and cognitive abilities (Trautwein, Lüdtke, Marsh et al., 2006), and instruction in this group achieves higher levels of cognitive activation. As a consequence of ability-grouping, performance standards are higher in academics track than in non-academic tracks. Thus, the academic track seems to provide qualitatively better conditions for academic learning. Furthermore, the role of social comparisons in the classroom may become more important in academic tracks than in non-academic-tracks.

The first investigation of the effect of tracking on students's self-concept during the transition from elementary school to secondary school in Germany was performed by Schwarzer, Lange and Jerusalem (1982). The self-concept of students who moved to the academic track was higher than the self-concept of students who moved to the non-academic track. After a few months in secondary school, the difference between the two groups' selfconcept decreased until the end of the first school year. Marsh et al. (2001) found the BFLPE occurred within a few weeks after the transition to the academic track. In another study, Watermann, Klingebiel, and Kurtz (2010) noted the BFLPE occurred for both students' selfconcept and their intrinsic motivation after the transition to the academic track.

Trautwein, Lüdtke, Marsh et al. (2006) found that the differences in students's selfconcept and motivation in the different school tracks can be caused by grades. Thus, students in high tracks (Gymnasium) had higher (better) grades than students in low tracks (Hauptschule and Realschule), and they also had a higher self-concept. This higher self-concept in high-achievement students may have been the result of higher grades. In this study of grade nine students, the authors found that controlling for grades reduced the effects of tracking on students' interest and nullified the effects of tracking on students' self-concept. According to these findings, Trautwein, Lüdtke, Marsh et al. (2006) concluded that differences in students' self-concept can be caused by grades. Consistent with Trautwein, Lüdtke, Marsh et al. (2006), Chiu et al. (2008) found that after controlling for students' grades in math tracking no longer affected seventh-grade students' math self-concept. Thus, grades are especially important when investigating the BFLPE.

Most previous research used standardized achievement tests as an indicator of students' level of achievement. Standardized tests were used because they are generally regarded as externally moderated and more objective than teacher-assigned grades. Südkamp and Möller (2009) showed that the reference group played an important role when students were 
graded. "Students with identical performance were graded more favorably in a class with low average achievement than in a class with higher average achievement," (Südkamp \& Möller, 2009, p. 161). Nevertheless, grades are highly important to students' academic self-concept (Skaalvik \& Skaalvik, 2002). Studies have shown that grades have a substantial effect on students' domain-specific academic self-concept and, conversely, that grades are likely to be affected by self-concept (e.g., Marsh \& Craven, 1997; Trautwein, Lüdtke \& Köller \& Baumert, 2006). In contrast to standardized achievement tests, which are an indicator of the overall standing of a group, teacher-assigned grades provide students with easily accessible feedback about their relative standing within a group. This information could be a basis of students's self-evaluation. The importance of grades as a main source of achievement feedback for students indicates that teacher-assigned grades may statistically mediate much of the effect of achievement on students' academic self-concept (Trautwein, Lüdtke, Marsh et al., 2006). Accordingly, the present study is designed to establish the potentially mediating effects of grades on the relationship between tracking and students'self-concept and motivation.

The studies described above mostly measured the effect of tracking on students's selfconcept, self-esteem, interest, educational aspirations or motivation. Some researchers have recently required an extension of the BFLPE on other psychological outcomes (Trautwein \& Lüdtke, 2005). Because social comparison is of great importance to the BFLPE, it is plausible that these effects could be found not only for students' self-concept, interest and motivation but also for their achievement goals. Achievement goals can be considered part of motivation. They have been found to be systematically related to students's self-concept, interest and intrinsic motivation. The present study is designed to investigate students' achievement goals in addition to students's self-concept and intrinsic motivation. The aim of the current study is to determine whether the BFLPE also affects achievement goals.

Achievement goals are defined as cognitive representations of competence-relevant possibilities that guide behavior to a competence-related end state that the individual is committed to either approaching or avoiding (Elliot \& Thrash, 2001; Hulleman et al., 2010). They are divided into two types of goals that individuals pursue in task/learning contexts: mastery goals and performance goals (Ames \& Ames, 1984). Additionally, the distinction between approach and avoidance is made for both mastery and performance goals (Elliot, 1999; Elliot \& McGregor, 2001). In contrast to mastery goals, which include attaining task-based competence or intrapersonal competence, performance goals are characterized by the importance of social comparisons. Performance goals can be further divided into performance-approach goals, which include attaining normative competence and demonstrating high competence, 
and performance-avoidance goals, which include avoiding normative incompetence and preventing negative judgments from others. In many correlational studies, mastery goals were positively related to students' self-concept, self-efficacy, intrinsic motivation, interest, and positive emotions (e.g., enjoyment) (e.g., Ames, 1992; Harackiewicz, Barron, Pintrich et al., 2002; Lemos, 1999; Rawsthorne \& Elliot, 1999). Converse results were frequently found for performance-avoidance goals (e.g., Linnenbrink, 2005; Middleton \& Midgley, 1997; Rawsthorne \& Elliot, 1999; Skaalvik, 1997), while the results for performance-approach goals were inconsistent.

Tracking negatively affects academically-tracked students' experience of competence and positively affects non-academically tracked students' experience of competence. This effect in turn influences students' motivation and self-efficacy (Deci, Vallerand, Pelletier, Ryan, 1991). Based on the effects noted above, it is plausible that academically tracked students have fewer mastery goals than non-academically tracked students because the mastery goals act as an expression of motivation and self-efficacy. Performance goals could also display a tracking effect because the social comparison processes are important for these types of achievement goals. Performance goals differ from mastery goals in that intrapersonal comparisons are more important for mastery goals than for performance goals. Social comparisons among students were the main reasons for contrast effects (Marsh, 1986). These comparisons could lead to a decrease in performance-approach goals and an increase in performanceavoidance goals in academic track students.

The research questions and hypotheses for the current study are as follows:

1. In accordance with the BFLPE, we expect differences in the development of self-concept and intrinsic motivation. Both variables should decrease for the academic track and slightly increase for the non-academic track.

2. Does the school track influence the development of achievement goals in the same way as it affects students' self-concept and intrinsic motivation? It is predicted that the academic track negatively influences the development of mastery goals. That is, development of mastery goals should decrease for the academic track, consistent with the predictions of BFLPE. For performance goals, a decrease is predicted in performance-approach goals, and an increase is predicted in performance-avoiding goals for academic tracks and vice-versa for non-academic-tracks.

3. Can the BFLPE on students' self-concept, intrinsic motivation, and achievement goals be explained by controlling not only for students' achievement in 
grade four but also for students' classroom grades in grade five? We expect that the BFLPE disappears or declines after controlling for students' grades after the transition.

\subsection{Method}

The empirical basis for the present investigation was the Transition Study, a cooperative project of the Max Planck Institute for Human Development in Berlin, the Department of Empirical Research in Education at the Freie Universität Berlin, the University of Potsdam, the Institute for School Development Research at the University of Dortmund, and the Institute for Educational Progress at the Humboldt University in Berlin. Germany participated in the 2007 Trends in Mathematics and Science Study (TIMSS) of students in the fourth grade [T1]. TIMSS has provided an ideal opportunity to extend scientific knowledge of how students and their parents cope with the process of transition. As such, two further waves of data collection were undertaken after the transition to secondary school. Data collection was conducted six months after transition, to coincide with the fifth grade mid-term report card [T2] and one year after transition, to coincide with the final fifth grade report card [T3]. The Transition Study was embedded within TIMSS 2007.

\subsubsection{Participants}

Its sample contained $N=1,646$ students from 246 schools (Gymnasium [academic track]: $n=928$, Realschule [middle track]: $n=436$, Hauptschule [low track]: $n=158$, other school types: $n=124$ ). This sample comprised most of the classes participating in TIMSS. Due to the small sample sizes of the middle and low track, the tracks and the other school types were combined into a non-academic track $(n=718)$. The sample included students who participated in grade four and in at least one of the other two occasions. The sample was 49.4 percent female, 87.4 percent Germany-born and had a mean age of 10.4 years $(S D=0.50)$ at T1.

\subsubsection{Measures}

Academic self-concept. To assess academic self-concept, three items of Marsh's Self Description Questionnaire (SDQ, 1990) were used, for example, "'I learn things quickly in 
most of the school subjects." The items were translated into German and had been used previously in PISA 2000 (Kunter et al., 2002) (Cronbach's $\alpha=.81$ ).

Intrinsic motivation. Intrinsic motivation was measured with four items (following Berlyne, 1978; Litman \& Spielberger, 2003) that were developed and tested for the Transition Study (Cronbach's $\alpha=.85$ ). An example of an intrinsic motivation item was "I enjoy exploring new ideas."

Achievement goals. Students were given surveys containing ten achievement goal items. The four mastery-approach goal items $(\alpha=.70)$ stemmed from the revised version of the Patterns of Adaptive Learning Scales (PALS) (Midgley et al., 2000). The items were translated into German and focused on developing academic competence, for example, "It it important to me that I improve my skills.” Mastery-avoidance goals were not measured, thus, the term mastery goals will refer only to mastery-approach goals. Performance-approach goals were measured with four items $(\alpha=.83)$ based on the German version of the Motivational Orientation Scales (MOS) (Köller \& Baumert, 1998; original version by Nicholls et al., 1985). The items focused on demonstrating high academic competence relative to classmates, e.g., "It is important to me that I know more than other students in my class." Performanceavoidance goals were assessed with two items $(\alpha=.59)$ based on items developed by Schwinger \& Wild (2006). The items focused on avoiding the demonstration of incompetence (appearance component), e.g., "It is important to me that others do not think I am stupid." All items were phrased generally and scored on a 4-point Likert-type scale $(1=$ very true through $4=$ not at all true). Items were reverse coded, so that high scores on the final scales indicated high levels of self-concept, intrinsic motivation and achievement goals.

Achievement. Students' achievement was assessed using the TIMSS Mathematics and Science Test (Mullis et al., 2005). The mathematics test consisted of 177 items (96 multiplechoice, 83 short answer format) and the science test consisted of 175 items (93 multiplechoice, 81 short answer format). The items were administered in a multi-matrix design and distributed over 14 blocks of tasks with 10 to 15 tasks per block. Each student were required to complete one test booklet containing two mathematics task blocks and two science task blocks. Each task block took approximately 20 minutes to complete. The tests were sufficiently reliable (Cronbach's $\alpha=.83$, respectively .80) and valid. Additionally, German achievement was assessed by the Institute for Educational Progress using a standardized German test (Cronbach's $\alpha=.81$ ). Students were required to complete two out of four task blocks. Task blocks included four competence areas: reading, listening, speaking, and writing. The task took approximately 40 minutes to complete. Achievement scores were computed using item 
response theory (IRT) techniques, resulting in a metric with a mean of 150 and a standard deviation of 10 .

Students' classroom grades in grades four and five were also used as a measure of performance attainment. GPA was computed for two time points (the middle of grades four and five) by taking the mean of four subjects (German, math, foreign language, social studies) in grade four and three subjects (German, math, foreign language) in grade five, respectively. The grades ranged from one to six; in which one was the best of all possible grades and six was the worst. Grades were reverse coded so that high scores on the final scales indicated high levels of achievement.

\subsubsection{Data analysis}

Analyses were conducted using the Mplus 6.11 program (Muthén \& Muthén, 19982011). Missing data were multiple imputed with Mplus (scale level). Multiple imputation is a procedure in which missing data are imputed based on the available information (Graham et al., 2003). Five complete data sets were created by filling in a random value for each missing data point. Mplus combined the results for each data set into an average result. Other treatments of missing data (e.g., listwise deletion, mean imputation) can lead to biased statistical inference (Little \& Rubin, 2002).

Growth curve analytic techniques were used to analyze the development of students' self-concept, intrinsic motivation, and achievement goals (Latent Growth Curve Models, LGCM; cf. Bollen \& Curran, 2006; Duncan et al., 2006; Meredith \& Tisak, 1990). Different unconditional models were compared with each other. LGCM is a tool for analyzing longitudinal data. It permits analysis of change on a latent level, by which random measurement errors are considered. Thus, inter-individual differences of change and different forms of change over time can be taken into account. The slope factor indicates the rate of change per time unit. The interpretation of the slope factor depends on the presumed time function. A linear growth per time unit is expected in a linear growth-curve model. However, this time function is not adequate for every case. In some cases, it can be appropriate to estimate a model with an unspecified growth trend (Meredith \& Tisak, 1990) in which the time function is not fixed a priori, but is estimated from the data. For each achievement goal, different growth-curve models were specified, beginning with a linear growth rate and followed by an unspecified (freed-loading LGCM) growth rate (Bollen \& Curran, 2006, Chap. 4). In the freed-loading LGCM, the first loading was fixed in the slope factor to 0 , the third loading was 
fixed to 1 , and the remaining second loading was freely estimated. The estimated value of the second factor loading indicates the amount of change occurring between measurement occasions one and two relative to the amount of change occurring between measurement occasions two and three. For example, a value of .25 means that 25 percent of the amount of change occurred between occasions one and two and 75 percent of the amount of change occurred between occasions two and three. In addition, equality restrictions were imposed on the residuals of the repeated measures. To test whether coefficients differed across the different school tracks, multi-group models were run for academic and non-academic tracks. To consider the nested structure of the data, the command type = general complex was used. The analyses were computed with the MLR estimator (maximum likelihood estimation with robust standard errors). Approximate fit indexes such as the comparative fit index (CFI), the Tucker-Lewis index (TLI), and the root mean square error of approximation (RMSEA) were considered to account for the increase in chi-square values due to increased sample size. A good fit is indicated with a value of greater than 0.95 for the CFI and TLI, and with a value smaller than 0.06 for the RMSEA (Hu \& Bentler, 1999; Yu, 2002).

To test whether track had an effect on the development of students's self-concept, intrinsic motivation, and achievement goals, conditional latent growth curve models were conducted with achievement in grade four (test scores and GPA) as the independent variable (Model M1). In the second model (M2), school track was introduced and in the third model (M3), GPA in grade five was included.

\subsection{Results}

Descriptive statistics were calculated for self-concept, intrinsic motivation, achievement goal variables, test scores, and GPAs. Table 9 provides the means and standard deviations of the different variables for the academic track and the non-academic track. The results revealed a decline in all variables for both tracks. The mean levels of self-concept, intrinsic motivation, and performance goals differed significantly between the two school tracks. Thus, school track appeared to affect the development of the different variables. The means for the test scores and GPAs indicated that students who moved to the academic track performed better than other students. 
Table 9

Descriptive statistics for self-concept, intrinsic motivation, achievement goal variables, test scores, and GPAs

\begin{tabular}{|c|c|c|c|c|}
\hline \multirow[b]{2}{*}{ Variable } & \multicolumn{2}{|c|}{ Academic track } & \multicolumn{2}{|c|}{ Non-academic track } \\
\hline & $M$ & $S D$ & $M$ & $S D$ \\
\hline Self-concept T1 & 3.67 & 0.42 & 3.24 & 0.57 \\
\hline Self-concept T2 & 3.32 & 0.54 & 3.21 & 0.56 \\
\hline Self-concept T3 & 3.26 & 0.53 & 3.14 & 0.55 \\
\hline Intrinsic motivation $\mathrm{T} 1$ & 3.12 & 0.67 & 2.89 & 0.72 \\
\hline Intrinsic motivation $\mathrm{T} 2$ & 2.75 & 0.64 & 2.80 & 0.64 \\
\hline Intrinsic motivation $\mathrm{T} 3$ & 2.55 & 0.64 & 2.55 & 0.67 \\
\hline Mastery goal T1 & 3.56 & 0.46 & 3.51 & 0.48 \\
\hline Mastery goal T2 & 3.46 & 0.46 & 3.44 & 0.47 \\
\hline Mastery goal T3 & 3.20 & 0.48 & 3.22 & 0.48 \\
\hline Performance-approach goal T1 & 2.43 & 0.87 & 2.71 & 0.82 \\
\hline Performance-approach goal T2 & 1.90 & 0.74 & 2.13 & 0.79 \\
\hline Performance-approach goal T3 & 1.71 & 0.67 & 1.91 & 0.76 \\
\hline Performance-avoidance goal T1 & 2.94 & 0.68 & 3.08 & 0.66 \\
\hline Performance-avoidance goal T2 & 2.50 & 0.66 & 2.68 & 0.65 \\
\hline Performance-avoidance goal T3 & 2.16 & 0.70 & 2.35 & 0.75 \\
\hline Test Score Math T1 & 157.42 & 8.20 & 148.83 & 7.96 \\
\hline Test Score Science T1 & 156.28 & 8.25 & 149.42 & 8.69 \\
\hline Test Score German T1 & 156.54 & 7.86 & 148.92 & 8.84 \\
\hline GPA T1 & 4.12 & 0.45 & 3.20 & 0.63 \\
\hline GPA T2 & 3.61 & 0.68 & 3.29 & 0.61 \\
\hline
\end{tabular}

Note. $\mathrm{T} 1=$ End of grade four. $\mathrm{T} 2=$ Middle of grade five. $\mathrm{T} 3=$ Beginning of grade six. $\mathrm{M}=$ Mean. SD = Standard Deviation. 


\subsubsection{Self-concept}

Before testing the effect of school track on students' self-concept, Latent Growth Curve Models (LGCM) were computed to analyze the patterns of development for students' self-concept. Results showed that the best model was a model with unspecified rate of change, and with equal factor loadings and residual variances (within the school track) but differing covariances $\left(X^{2}=16.93, d f=5, \mathrm{RMSEA}=.053, \mathrm{CFI}=.987\right.$, TLI $\left.=984\right)$. Of particular interest, approximately 90 percent of the decline in students's self-concept occurred during the first half of the first year of secondary school.

The results of the conditional LGCMs are displayed in Table 10. As frequently indicated in previous research, individual achievement (GPA) influenced the initial level in grade four and the rate of change in students's self-concept (M1). Students with better GPAs in grade four had a higher self-concept at the end of elementary school but a greater decline in selfconcept after the transition. School track was included in the second model (M2). The results revealed that school track (academic track) positively influenced the intercept factor of students's self-concept and negatively influenced the slope factor. Students who moved to the academic track had a higher self-concept before the transition, but their self-concept declined more after the transition compared with the self-concept of students who moved to the nonacademic track. This effect is an indicator for the BFLPE in the academic track. GPA for grade five was included in the third model (M3). Interestingly, the BFLPE did not disappear after controlling for GPA in grade five. Although the slope coefficient decreased substantially, it remained significant. The test scores were not very important for the initial level or the rate of change in students's self-concept. In contrast, GPA was the most influential predictor for students' self-concept. Furthermore, GPA partially mediated the relationship between school track and students`s self-concept. 
Table 10

Results of the conditional latent growth curve models for students'self-concept

\begin{tabular}{|c|c|c|c|c|c|c|c|c|c|c|c|c|}
\hline & \multicolumn{4}{|l|}{ M1 } & \multicolumn{4}{|l|}{ M2 } & \multicolumn{4}{|l|}{ M3 } \\
\hline & \multicolumn{2}{|l|}{ Intercept } & \multicolumn{2}{|l|}{ Slope } & \multicolumn{2}{|l|}{ Intercept } & \multicolumn{2}{|l|}{ Slope } & \multicolumn{2}{|l|}{ Intercept } & \multicolumn{2}{|l|}{ Slope } \\
\hline & $B$ & $S E$ & $B$ & $S E$ & $B$ & $S E$ & $B$ & $S E$ & $B$ & $S E$ & $B$ & $S E$ \\
\hline Test Score Math T1 & -.003 & .002 & $.007 * * *$ & .002 & $-.003^{*}$ & .002 & $.009 * * *$ & .002 & $-.003^{*}$ & .002 & .004 & .002 \\
\hline Test Score Science T1 & .001 & .002 & .000 & .002 & .000 & .002 & .000 & .002 & .000 & .002 & .001 & .002 \\
\hline GPA T1 & $.388 * * *$ & .026 & $-.224 * * *$ & .032 & $.316^{* * *}$ & .028 & $-.087 *$ & .038 & $.314 * * *$ & .031 & $-.219 * * *$ & .035 \\
\hline GPA T2 & & & & & & & & & .016 & .021 & $.372 * * *$ & .023 \\
\hline $\mathrm{R}^{2}$ & \multicolumn{2}{|c|}{$.423(.040)^{* * *}$} & \multicolumn{2}{|c|}{$.091(.024) * * *$} & \multicolumn{2}{|c|}{$.420(.035) * * *$} & \multicolumn{2}{|c|}{$.169(.031)^{* * *}$} & \multicolumn{2}{|c|}{$.435(.037)^{* * *}$} & \multicolumn{2}{|c|}{$.465(.057)^{* * *}$} \\
\hline
\end{tabular}

Note. Track: academic track (1), non-academic track (0). Unstandardized coefficients.

$* p<.05 . * * p<.01 . * * * p<.001$. 


\subsubsection{Intrinsic motivation}

The results of the LGCMs for students' intrinsic motivations revealed that the best fit model was an unspecified model with equated residual variances over time, but differing factor loadings (intercept and slope) and covariances across the school tracks $\left(X^{2}=10.03, d f=3\right.$, RMSEA $=.053, \mathrm{CFI}=.991, \mathrm{TLI}=982)$. The factor loading of $0.64(\mathrm{~T} 2)$ for academic track revealed that 64 percent of the change in students' intrinsic motivation occurred during the first half of the school year for students in the academic track, whereas the factor loading for the non-academic track was much smaller (0.38).

In a further step, conditional LGCMs were conducted (see Table 11). The results of M1 revealed that GPA in grade four positively influenced the initial level of students' intrinsic motivation and negatively influenced the rate of change. Students with higher GPAs in grade four experienced higher intrinsic motivation before the transition and a greater decrease in motivation after the transition. The results of the second model showed the BFLPE for the academic track. Students who moved to the academic track were more motivated at T1, but their motivation declined more after the transition. This effect remained significant and almost unchanged after controlling for students' GPA in grade five. Test scores did not play a significant role in the development of students' intrinsic motivation. 
Table 11

Results of the conditional latent growth curve models for students' intrinsic motivation

\begin{tabular}{|c|c|c|c|c|c|c|c|c|c|c|c|c|}
\hline & \multicolumn{4}{|l|}{ M1 } & \multicolumn{4}{|l|}{ M2 } & \multicolumn{4}{|l|}{ M3 } \\
\hline & \multicolumn{2}{|l|}{ Intercept } & \multicolumn{2}{|l|}{ Slope } & \multicolumn{2}{|l|}{ Intercept } & \multicolumn{2}{|l|}{ Slope } & \multicolumn{2}{|l|}{ Intercept } & \multicolumn{2}{|l|}{ Slope } \\
\hline & $B$ & $S E$ & $B$ & $S E$ & $B$ & $S E$ & $B$ & $S E$ & $B$ & $S E$ & $B$ & $S E$ \\
\hline Test Score Math T1 & -.002 & .003 & .002 & .003 & -.003 & .002 & .004 & .003 & -.005 & .002 & .004 & .003 \\
\hline Test Score Science T1 & .003 & .002 & -.001 & .003 & .002 & .002 & .004 & .003 & .002 & .002 & .001 & .003 \\
\hline GPA T1 & $.153 * * *$ & .033 & $-.146^{* *}$ & .052 & $.121^{* *}$ & .041 & $-.113^{*}$ & .046 & $.093 *$ & .043 & $-.141^{*}$ & .058 \\
\hline GPA T2 & & & & & & & & & $.100 * * *$ & .050 & .014 & .035 \\
\hline $\mathrm{R}^{2}$ & \multicolumn{2}{|c|}{$.037(.013)^{* *}$} & \multicolumn{2}{|c|}{$.065(.028)^{*}$} & \multicolumn{2}{|c|}{$.045(.014) * * *$} & \multicolumn{2}{|c|}{$.089(.025)^{* * *}$} & \multicolumn{2}{|c|}{$.063(.015)^{* * *}$} & \multicolumn{2}{|c|}{$.107(.029)^{* * *}$} \\
\hline
\end{tabular}

Note. Track: academic track (1), non-academic track (0). Unstandardized coefficients.

$* p<.05 . * * p<.01 . * * * p<.001$. 


\subsubsection{Mastery goals}

The findings of the LGCMs for mastery goals revealed that the best fit model was an unspecified model with equated factor loadings (intercept and slope) and residual variances across the school tracks $\left(X^{2}=5.09, d f=4, p>.05\right.$, RMSEA $=.016$, CFI $=.998$, TLI $\left.=.997\right)$. The slope loading $(0.28)$ to T2 revealed that 28 Prozent of the change in students' mastery goals occurred during the first half of the first year of secondary school.

The results of the conditional LGCMs (Table 12) revealed that the test score in math was negatively related to the initial level of students' mastery goals in grade four (M1 to M3). Furthermore, GPA was positively related to the intercept factor. The better students' GPAs were in grade four, the more oriented they were toward mastery goals before the transition and vice versa. School track was included in model 2. Academic track negatively influences the slope factor, indicating that students' mastery goals declined more when moving to the academic track than when moving to the non-academic track. This effect disappeared after controlling for the GPA in grade five. After controlling for GPA in grade five, school track influenced the initial level positively. There were no significant effects of test scores or GPAs on the rate of change in students' mastery goals. 
Table 12

Results of the conditional latent growth curve models for students' mastery goals

\begin{tabular}{|c|c|c|c|c|c|c|c|c|c|c|c|c|}
\hline & \multicolumn{4}{|l|}{ M1 } & \multicolumn{4}{|l|}{ M2 } & \multicolumn{4}{|l|}{ M3 } \\
\hline & \multicolumn{2}{|l|}{ Intercept } & \multicolumn{2}{|l|}{ Slope } & \multicolumn{2}{|c|}{ Intercept } & \multicolumn{2}{|l|}{ Slope } & \multicolumn{2}{|c|}{ Intercept } & \multicolumn{2}{|l|}{ Slope } \\
\hline & $B$ & $S E$ & $B$ & $S E$ & $B$ & $S E$ & $B$ & $S E$ & $B$ & $S E$ & $B$ & $S E$ \\
\hline Test Score Math T1 & $-.004 *$ & .001 & .002 & .002 & $-.004 *$ & .002 & .001 & .002 & $-.004 * *$ & .002 & .001 & .002 \\
\hline Test Score Science T1 & -.001 & .002 & -.004 & .002 & -.001 & .002 & -.004 & .002 & -.001 & .002 & -.004 & .002 \\
\hline GPA T1 & $.063 * *$ & .020 & -.003 & .037 & $.054 *$ & .025 & .035 & .038 & .029 & .025 & .037 & .045 \\
\hline GPA T2 & & & & & & & & & $.048 * *$ & .019 & .004 & .028 \\
\hline $\mathrm{R}^{2}$ & \multicolumn{2}{|c|}{$.037(.013)^{* *}$} & \multicolumn{2}{|c|}{$.065(.028)^{*}$} & \multicolumn{2}{|c|}{$.045(.014) * * *$} & \multicolumn{2}{|c|}{$.089(.025)^{* * *}$} & \multicolumn{2}{|c|}{$.063(.015)^{* * *}$} & \multicolumn{2}{|c|}{$.107(.029)^{* * *}$} \\
\hline
\end{tabular}

Note. Track: academic track (1), non-academic track (0). Unstandardized coefficients.

$* p<.05 . * * p<.01 . * * * p<.001$. 


\subsubsection{Performance-approach goals}

The results of the LGCMs indicated that the best fit model was an unspecified model with equated factor loadings (intercept and slope) and residual variances, but differing covariances across the school tracks $\left(X^{2}=18.97, d f=4, \mathrm{p}<.001, \mathrm{RMSEA}=.067, \mathrm{CFI}=.984\right.$, TLI $=.976)$. The model parameters revealed that 73.3 Prozent of the change in students' performance-approach goals occurred during the first half of the first year of secondary school.

The results of the conditional LGCMs (Table 13) indicated that school track did not influence the initial level or the rate of change of students's performance-approach goals. Effects of test scores were only found for math (M1 and M2) and German (M1-M3). GPA in grades four and five affected the intercept factor, revealing that the better students's school achievement was, the lower their performance-approach goals were and vice versa. There were no effects on the development of performance-approach goals.

\subsubsection{Performance-avoidance goals}

For performance-avoidance goals, the best fit model was an unspecified model with equated trajectories and residual variances across both repeated measures and groups $\left(X^{2}=\right.$ $8.71 d f=6, \mathrm{p}>.05, \mathrm{RMSEA}=.022, \mathrm{CFI}=.996, \mathrm{TLI}=.996)$. The model parameters showed that 55.5 percent of the change in students' performance-avoidance goals occurred during the first half of the first year of secondary school.

The results of the conditional LGCMs with school achievement and school track as predictors indicated that the school track did not influence the initial level or the rate of change in students' performance-avoidance goals (see Table 14). In contrast to the other examined variables, GPAs did not predict the intercept or slope factor of performance-avoidance goals. Only the test scores in math and German negatively predicted the intercept of performance-avoidance goals in grade four (M1 and M2). There were no significant effects on the development of this type of achievement goal. 
Table 13

Results of the conditional latent growth curve models for students' performance-approach goals

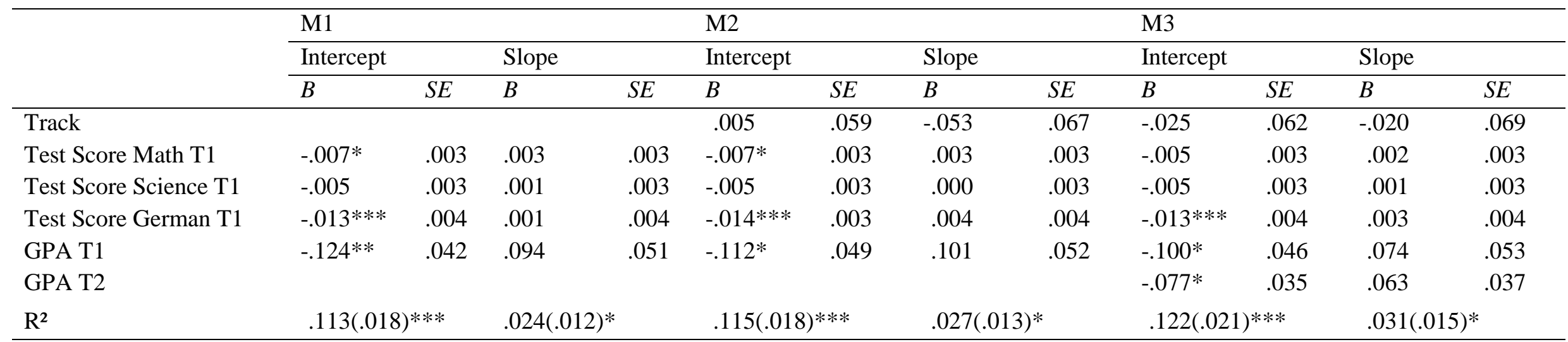

Note. Track: academic track (1), non-academic track (0). Unstandardized coefficients.

$* p<.05 . * * p<.01 . * * * p<.001$.

Table 14

Results of the conditional latent growth curve models for students' performance- avoidance goals

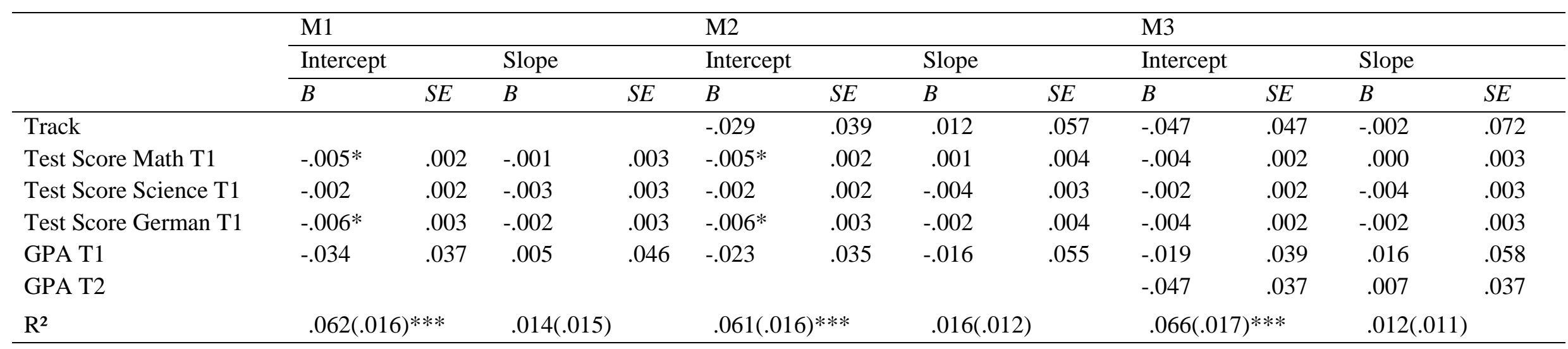

Note. Track: academic track (1), non-academic track (0). Unstandardized coefficients.

$* p<.05 . * * p<.01 . * * * p<.001$. 


\subsection{Discussion}

The present study examined the effect of between-school tracking not only on students's self-concept and intrinsic motivation but also on students' achievement goals. Previous literature frequently noted a contrast effect for students'self-concept and intrinsic motivation. Could this effect also be found for achievement goals? The authors predicted that social comparison processes should impact not only the development of students's self-concept and intrinsic motivation but also achievement goals because social comparisons are especially important in particular for performance goals. If the mechanism is correctly predicted, then the BFLPE should also be found for students' achievement goals.

The German school system is particularly useful for investigating the effects of tracking on achievement goals because the segregation of students into different school tracks after grade four is based partly on their academic abilities. The academic track is the most valued school track among the different school types, and the learning group is positively selected in terms of cognitive abilities. The current analyses confirmed that students who moved to the academic track scored approximately one standard deviation higher on standardized tests than students who moved to the non-academic track. It is reasonable to assume that the selfconcept and intrinsic motivation of students in non-academic tracks would be lower than selfconcept and intrinsic motivation in academic tracks due to the high visibility of the tracking structure and the differences in achievement between the two groups. The current study's results indicated that students in the non-academic track had a lower self-concept and intrinsic motivation than students in the academic track.

The research question referred to the tracking effect on students' achievement goals. Before analyzing this question, we replicated the findings of other studies (e.g., Marsh et al., 2001; Trautwein, Lüdtke, Marsh et al., 2006, Watermann et al., 2010). The BFLPE on students's self-concept and intrinsic motivation occurred after the transition to the academic track. Academic track students experienced more decline in self-concept and intrinsic motivation than non-academic track students. It was hypothesized that the BFLPE would also be found for achievement goals. The results of the conditional growth curve models revealed that the initial level of mastery goals at the end of grade four was higher for students who later moved to the academic track than for students who moved to the non-academic track. Furthermore, the school track negatively influenced the development of mastery goals after the transition (M2). Interestingly, no significant tracking effects were found on the development of mastery goals after controlling for students' GPA in grade five, although a non-significant trend to- 
ward a contrast effect was found in the academic track. Mastery goals declined more in the academic track than in the non-academic track, although this effect was not significant (M3).

There were no indications of a contrast effect for performance goals. The school track did not significantly affect the initial level or the development of performance-approach and performance-avoidance goals. Performance goals decreased both for students in the academic and the non-academic tracks. Furthermore, mastery goals did not increase in the nonacademic track. Thus, the school track in itself did not appear to impact the development of achievement goals (especially performance goals) at this age. It is plausible that the learning environment of secondary schools in general caused the decrease in motivation or achievement goals. Studies on the stage-environment fit theory (Eccles et al., 1993) indicated that changes in classroom variables contribute to the decline in students' motivation. These changes could have resulted in a developmental mismatch between early adolescents' needs and the classroom environment. On the one hand, this mismatch may have caused a decline in achievement goals as a motivational outcome. On the other hand, the decline in students' achievement goals could be a result of developmental changes independent of the learning environment. Future research should investigate this question in more depth.

The second research question referred to the mediating effect of classroom grades on the relationship between tracking and dependent variables such as self-concept and achievement goals. Unlike prior studies, grades were included in the analyses and it was assumed that the tracking effect may be mediated by grading practices. Does the BFLPE disappear after controlling for students' achievement after the transition (GPA)? The results of the analyses suggest that the contrast effect on students's self-concept declined but remained significant and apparent after controlling for students' GPA after the transition to secondary school in grade five. This result indicated that the predictive effect of individual achievement was partially mediated by teacher-assigned grades. Thus, the differential grading practices in the different school tracks appear to have partially affected the development of students'self-concept.

The results for intrinsic motivation revealed that after controlling for students' achievement after the transition, the BFLPE remained almost the same. This result indicated that, given comparable individual achievement, the school track still influenced the development of students' intrinsic motivation. The motivation of academic track students decreased more than the motivation of non-academic track students. Thus, the low motivation in the academic track does not appear to be a consequence of differential grading practices.

Interestingly, academic track students' intrinsic motivation declined much more quickly $(64 \%)$ than the intrinsic motivation of non-academic track students (38\%). GPA in grade 
five did not explain the BFLPE on students' intrinsic motivation, thus other factors must have caused this effect. The transition to the secondary school and its attendant changes seem to have impacted the development of intrinsic motivation, especially for the academic track students (stage-environment fit; Eccles et al., 1993).

The BFLPE on students' mastery goals disappeared after controlling for students' GPA in grade five. Thus, the differential grading practices in the different school tracks seem to have affected the development of mastery goals. Given comparable achievement, academic track students' mastery goals did not decline more than non-academic track students' mastery goals.

The present study replicates previous research on the effect of tracking on students' self-concept and intrinsic motivation and extends this research to include students' achievement goals. Although the results of the current study indicated that contrast effects do not play a significant role in the development of achievement goals (especially performance goals), further research on the development of achievement goals could focus on identifying the mediating variables that explain the decline in the different types of achievement goals. The current results suggest that achievement goals seem to be conceptually different from intrinsic motivation because no tracking effect was observed on students' achievement goals. However, achievement goals were much less affected by teacher-assigned grades than were students' self-concept and intrinsic motivation. Future research should identify the factors that influence the development of achievement goals to counteract this decline.

There are a few limitations that need to be considered. In the current study, students' self-concept, intrinsic motivation and achievement goals were measured domain-general, but not domain-specific for each school subject. Thus, it is possible that the development of the different variables and the tracking effects differs between the individual school subjects.

Furthermore, since the interval between the occasions was relatively large (approximately six months), we cannot state clearly when the BFLPE occurred. Other studies showed that the contrast effect occurs shortly after the transition to secondary school (e.g., Aust et al., 2010). It could be possible that there was a tracking effect on students' performance goals, but that this effect occurred shortly after the transition and disappeared in the following months. A micro genetic perspective with many occasions short time after the transition could solve this problem. Future research should keep this in mind when investigating tracking effects on different variables.

Overall, the present study revealed that the school track plays a significant role in the development of students' self-concept, intrinsic motivation, and mastery goals. However, the 
differential grading practices in the different school tracks mediated the tracking effects on students' mastery goals, and partially on students's self-concept. 


\section{$7 \quad$ Zusammenfassende Schlussdiskussion und Ausblick}

Im folgenden Kapitel werden zunächst die Forschungsfragen der vorliegenden Arbeit aus Kapitel 3.1 wieder aufgegriffen und die in Studie 1 bis 4 gefundenen Ergebnisse zusammengefasst (7.1), bevor in Abschnitt 7.2 die zentralen Ergebnisse integrativ diskutiert werden. Abschließend werden die Grenzen der Arbeit aufgezeigt und Fragen für zukünftige Forschung abgeleitet (7.3).

\subsection{Zusammenfassung der zentralen Ergebnisse}

Das Ziel der hier präsentierten Arbeit war es, Zielorientierungen am Grundschulübergang mit einer für die Bundesrepublik für die vierte Klassenstufe repräsentativen Stichprobe zu untersuchen. Im Rahmen von vier Studien sollten folgende Fragen beantwortet werden:

1. Lassen sich Zielorientierungen bereits am Ende der Grundschulzeit reliabel und valide messen und

2. in welchem Zusammenhang stehen Zielorientierungen mit schulischen Leistungen am Grundschulübergang?

3. Unterscheiden sich die Zusammenhänge, wenn curricular valide Testleistungen bzw. Ziffernnoten verwendet werden?

4. Wie entwickeln sich Zielorientierungen am Grundschulübergang und

5. lassen sich schulformspezifische Unterschiede feststellen?

6. In welchem Zusammenhang stehen Zielorientierungen mit schulischen Leistungen vor und nach dem Grundschulübergang?

7. Wirkt sich der Bezugsgruppenwechsel ähnlich wie beim Selbstkonzept und der intrinsischen Motivation auch bei den Zielorientierungen aus und

8. lässt sich der BFLPE durch die Kontrolle der schulischen Leistungen in Klassenstufe fünf erklären?

Die zentralen Ergebnisse zu Fragen 1, 2 und 3 beziehen sich auf die Befunde der in Kapitel 4 dargestellten Studien 1 und 2. Studie 3 (Kapitel 5) liefert Antworten auf die Fragen 4 bis 6 und Studie 4 (Kapitel 6) wird zur Beantwortung der Frage 7 und 8 herangezogen. 
1. Lassen sich Zielorientierungen bereits in der Grundschule reliabel und valide messen?

Die Analyse der Faktorstruktur von Zielorientierungen am Ende der Grundschulzeit in Klassenstufe vier mittels konfirmatorischer Faktorenanalysen zeigte die theoretisch postulierte 3-Faktorstruktur. Demnach lassen sich die drei Formen von Zielorientierungen (Lern-, Annäherungsleistungs- und Vermeidungsleistungsziele) bereits bei sehr jungen Schülern in der Grundschule faktoriell valide messen. Allerdings deuteten die Analysen zur Konstruktvalidität auf eine eingeschränkte Validität des eingesetzten Instruments (modifizierte Version des PALS) hin. Während die Validitätskoeffizienten für die Lernziele insbesondere im Hinblick auf das Fähigkeitsselbstkonzept und die intrinsische Motivation erwartungsgemäß ausfielen, zeigten Vermeidungsleistungsziele teilweise unerwartete Zusammenhangsmuster. So konnte ein geringer positiver Zusammenhang der Vermeidungsleistungsziele mit dem Selbstkonzept und der Motivation nachgewiesen werden. Annäherungsleistungsziele hingen sowohl mit intrinsischer Motivation als auch mit Leistungsangst gering positiv zusammen.

2. In welchem Zusammenhang stehen Zielorientierungen mit schulischen Leistungen am Grundschulübergang?

In Studie 1 zeigte sich allein für Annäherungsleistungsziele ein praktisch bedeutsamer Zusammenhang zu schulischen Leistungen. Hohe Annäherungsleistungsziele gingen mit schlechteren Schulleistungen einher. Anders als erwartet, waren Lernziele kaum prädiktiv für Schulleistungen und auch Vermeidungsleistungsziele standen in keinem signifikanten Verhältnis zu Testleistungen bzw. Schulnoten.

Da der Zusammenhang zwischen Annäherungsleistungszielen und Schulleistungen wider Erwarten negativ war und ausgeschlossen werden sollte, dass es sich bei diesem Ergebnis um einen für das eingesetzte Instrument spezifischen Befund handelt, wurde in Studie 2 anhand einer anderen Stichprobe und eines anderen Instruments zur Erfassung von Zielorientierungen (SELLMO) eine Replikation des unerwarteten Ergebnisses aus Studie 1 angestrebt. Auch in Studie 2 konnte ein negativer Zusammenhang zwischen Annäherungsleistungszielen und Schulleistungen nachgewiesen werden, allerdings verschwand dieser Zusammenhang für die Schulnoten nach Kontrolle der anderen Dimensionen der Zielorientierungen und der kognitiven Grundfähigkeit. Zudem hingen in Studie 2 Lernziele positiv mit der Leseleistung und 
Vermeidungsleistungsziele - bei bivariater Betrachtung - negativ mit Schulleistungen zusammen.

3. Unterscheiden sich die Zusammenhänge zwischen Zielorientierungen und schulischen Leistungen, wenn curricular valide Schulleistungstests bzw. Ziffernnoten verwendet werden?

Da es in Studie 2 nicht möglich war, an beiden untersuchten Schulen die Noten der Schüler zu erheben, basiert die Antwort dieser Frage auf den Ergebnissen der ersten Studie. Es hat sich gezeigt, dass Lernziele ein wenig enger mit den Noten und Annäherungsleistungsziele ein wenig enger mit der Mathematikleistung zusammenhingen. Die Unterschiede waren allerdings gering.

4. Wie entwickeln sich Zielorientierungen am Grundschulübergang?

Die Ergebnisse der Studie 3 belegen ein generelles Absinken aller drei Formen von Zielorientierungen von Klassenstufe vier bis sechs sowohl am Gymnasium als auch an den anderen Schulformen. Dabei zeigten Leistungsziele - insbesondere Annäherungsleistungsziele - einen Großteil der Veränderung (73,3 \% bzw. 55,5 \% bei Vermeidungsleistungszielen) am Grundschulübergang, zwischen dem ersten und dem zweiten Messzeitpunkt (T1: Ende Klassenstufe vier, T2: Mitte Klassenstufe fünf). Hingegen verzeichneten Lernziele lediglich 27,8 Prozent ihrer Veränderung während des ersten Halbjahres in Klassenstufe fünf.

5. Lassen sich schulformspezifische Unterschiede in der Entwicklung der Zielorientierungen finden?

Es ergaben sich lediglich geringe schulformspezifische Unterschiede in der Entwicklung der Zielorientierungen. Schüler, die auf ein Gymnasium wechselten, waren zu allen drei Messzeitpunkten etwas weniger leistungszielorientiert und ein wenig mehr lernzielorientiert am Ende der Grundschulzeit als Schüler, die auf eine der anderen Schulformen wechselten. Die Befunde - insbesondere der latenten Wachstumskurvenmodelle - deuten insgesamt darauf hin, dass sich die verschiedenen Formen von Zielorientierungen an den jeweiligen Schulformen ähnlich entwickeln. 
6. In welchem Zusammenhang stehen Zielorientierungen mit schulischen Leistungen vor und nach dem Übergang zu den verschiedenen Schulformen?

In der vorliegenden Arbeit wurden die jeweiligen Ergebnisse der einzelnen Schulformen miteinander verglichen. So wurden die Schüler der Haupt-, Real- und Gesamtschulen zu einer Gruppe zusammengefasst und mit den Schülern der Gymnasien verglichen. Die Ergebnisse des Cross-Lagged Panel Modells bestätigen die Hypothese eines positiven Zusammenhangs zwischen Lernzielen und Schulnoten am Ende der Grundschulzeit. Bei gleichzeitiger Kontrolle der Leistungsziele ging eine hohe Lernzielorientierung in der Mitte von Klassenstufe vier mit besseren Schulnoten am Ende der vierten Klassenstufe einher. Dieser positive Zusammenhang zeigte sich auch nach dem Grundschulübergang sowohl am Gymnasium als auch an den anderen Schulformen. Wie erwartet, bestand eine negative Verbindung zwischen Annäherungsleistungszielen und Schulnoten in der Grundschule. Diese Form der Zielorientierung ging demnach mit schlechteren schulischen Leistungen einher. Auch nach dem Grundschulübergang blieb dieser negative Zusammenhang bei Schülern, welche auf ein Gymnasium wechselten, bestehen. Vermeidungsleistungsziele erwiesen sich zu keinem Messzeitpunkt als bedeutsamer Prädiktor schulischer Leistungen an den jeweiligen Schulformen.

7. Beeinflusst die Schulform die Entwicklung der Zielorientierungen ähnlich wie es beim Fähigkeitsselbstkonzept und der intrinsischen Motivation der Fall ist? Lässt sich der Big-Fish-Little-Pond-Effekt (BFLPE) auch bei Zielorientierungen nachweisen?

Bevor dieser Frage in Studie 4 nachgegangen wurde, wurden die Ergebnisse der Untersuchung von Watermann et al. (2010) repliziert, so zeigten konditionale latente Wachstumskurvenmodelle den BFLPE nach dem Übergang von der Grundschule auf das Gymnasium sowohl für das Fähigkeitsselbstkonzept der Schüler als auch für die intrinsische Motivation. Entgegen den Erwartungen konnte kein signifikanter Kontrasteffekt auf die verschiedenen Leistungsziele und lediglich ein geringer BFLPE auf die Lernziele nachgewiesen werden.

8. Kann der BFLPE durch Kontrolle der schulischen Leistungen vor sowie nach dem Übergang erklärt werden? Verschwindet der BFLPE bei vergleichbaren Schulnoten in Klassenstufe fünf? 
Der negative Kontrasteffekt auf das Fähigkeitsselbstkonzept von Schülern, welche auf ein Gymnasium wechselten, verringerte sich deutlich nach zusätzlicher Kontrolle der Schulnoten nach dem Übergang. Dennoch verschwand dieser nicht, sondern blieb weiterhin signifikant. Es konnte eine partielle Mediation der Schulnoten in Klassenstufe fünf auf den BFLPE nachgewiesen werden. Im Gegensatz dazu änderte sich an der Größe des BFLPEs auf die intrinsische Motivation der Schüler nach zusätzlicher Kontrolle der Schulnoten in der fünften Klassenstufe kaum. Hingegen verschwand der negative Einfluss der Schulform auf die Entwicklung der Lernziele nach Kontrolle der Schulnoten in Klassenstufe fünf. 


\subsection{Integrative Diskussion}

Ziel der vorliegenden Arbeit war die Untersuchung von Zielorientierungen und deren Auswirkungen auf schulisches Lernen am Übergang von der Grundschule auf die weiterführende Schule. Die TIMS-Studie 2007 bot eine gute Gelegenheit, die an der Studie beteiligten Schüler auch nach der Erhebung in der vierten Klassenstufe zu begleiten und diese nach dem Grundschulübergang zu verschiedenen Konstrukten zu befragen. Der Übergang auf die weiterführende Schule stellt als ein kritisches Lebensereignis dar, da dieser mit zahlreichen Veränderungen einhergeht. Insbesondere im deutschsprachigen Raum sind keine Untersuchungen zur Übergangsbewältigung an einer repräsentativen Stichprobe bekannt, daher soll dem Konstrukt der Zielorientierung am Übergang zur weiterführenden Schule mehr Aufmerksamkeit geschenkt werden. Um die Forschungslücke zu schließen, bot sich die Untersuchung der motivationalen Bewältigung des Grundschulübergangs anhand der TIMSS-Übergangsstudie an. Da Zielorientierungen einen Teil der Motivation darstellen und sie sich in zahlreichen Studien als bedeutsam in Bezug auf schulisches Lernen erwiesen haben, diese jedoch bislang in nur unzureichendem Maße am Grundschulübergang in der Bundesrepublik untersucht wurden, wurde das Hauptaugenmerk der vorliegenden Untersuchung auf Zielorientierungen von Schülern der vierten bis sechsten Klassenstufe gelegt. Dabei stellen die vorgestellten Studien eine Erweiterung zu bisherigen Studien in zahlreicher Hinsicht dar. Zum einen wurden Zielorientierungen bei vergleichsweise jungen Schülern am Ende der Grundschulzeit an einer für die Bundesrepublik Deutschland repräsentativen Stichprobe erhoben (1), zum anderen wurden Zielorientierungen und deren Zusammenhang mit schulischen Leistungen im Längsschnitt über insgesamt drei Erhebungszeitpunkte erfasst, was die Betrachtung der Entwicklung von Zielorientierungen ermöglichte. Zudem wurden - anders als in vielen bisherigen Untersuchungen - zusätzlich zu Ziffernnoten objektive Testleistungen in Form von curricular validen Leistungstests in Klassenstufe vier erfasst, was die differenzielle Betrachtung der Zusammenhänge zwischen Zielorientierungen und schulischen Leistungen ermöglichte. Außerdem war aufgrund der Begleitung der Schüler nach dem Übergang auf die verschiedenen Schulformen eine schulformspezifische Betrachtung der Entwicklung von Zielorientierungen und deren Zusammenhang zu schulischen Leistungen möglich (2). Des Weiteren ermöglichte die Befragung von Schülern der verschiedenen Schulformen die Untersuchung von Bezugsgruppeneffekten zum einen auf Zielorientierungen und zum anderen auch auf andere im Rahmen der Untersuchung erhobenen motivationalen Merkmale (Fähigkeitsselbstkonzept und intrinsische 
Motivation) (3). Im Folgenden sollen die drei für die vorliegende Arbeit zentralen Aspekte in Bezug auf deren Ertrag, den sie für die Arbeit erbracht haben, diskutiert werden.

\section{(1) Untersuchung von Zielorientierungen am Grundschulübergang}

Ein zentraler Punkt der vorliegenden Arbeit war die Untersuchung von Zielorientierungen bereits in der Grundschule an einer für die Bundesrepublik Deutschland repräsentativen Stichprobe. Damit wird an bisherige Untersuchungen von Zielorientierungen in der Grundschule angeknüpft (für den deutschsprachigen Raum: Finsterwald, 2006; Schwinger \& Wild, 2006), welche sich allerdings zum einen auf homogene Stichproben und zum anderen im Falle von Schwinger und Wild auf die bereichsspezifische Erfassung von Zielorientierungen beschränkt haben. Bis dato ist keine deutschsprachige Untersuchung bekannt, welche sich dem Thema Zielorientierungen in der Grundschule an einer repräsentativen Stichprobe gewidmet hat. Somit stellte sich für die vorliegende Arbeit zuallererst die Frage, inwieweit sich Zielorientierungen bei so jungen Schülern reliabel und valide messen lassen. Ein weiterer Unterschied zu bisherigen Untersuchungen stellte die Erhebung curricular valider Testleistungen zusätzlich zu den Ziffernnoten dar. Anders als bei standardisierten Leistungstests wird die Notenvergabe als weniger objektiv bewertet, da nicht nur die tatsächlichen schulischen Leistungen der Schüler bei der Vergabe der Noten einfließen, sondern darüber hinaus auch Aspekte der Motivation sowie des Arbeits- und Lernverhaltens. Neben der Reliabilität und Konstruktvalidität von Zielorientierungen am Ende der Grundschulzeit war deren Zusammenhangsmuster zu schulischen Leistungen in Form von Ziffernnoten und Testleistungen eine wenig erforschte Frage. Die Zahl der Untersuchungen insbesondere im angloamerikanischen Raum zum Zusammenhang zwischen Zielorientierungen und Schulleistungen ist zwar umfangreich, allerdings bezieht sich nur ein geringer Teil der Studien auf Grundschüler. Zudem ist die Befundlage der Studien in dieser Altersgruppe inkonsistent.

Die Ergebnisse der vorliegenden Arbeit zu Zielorientierungen in der Grundschule belegen insgesamt die Reliabilität und in vielen Teilen Validität der modifizierten Kurzversion der PALS bei Schülern am Ende von Klassenstufe vier (Kapitel 4, Studie 1). So konnte zum einen die theoretisch angenommene faktorielle Struktur der drei Formen der Zielorientierungen und zum anderen die Reliabilität der Skalen bestätigt werden. Trotz der geringen Anzahl der Items (insbesondere für die Vermeidungsleistungsziele) kann die Reliabilität als zufriedenstellend bis sehr gut bewertet werden. Somit gliedert sich dieses Ergebnis in die Befunde 
der Metaanalyse von Hulleman et al. (2010) ein, wonach Zielorientierungen auch schon im Primarbereich reliabel gemessen werden können.

Allerdings deuten die Analysen zur Konstruktvalidität auf eine eingeschränkte Validität der Vermeidungsleistungsziele hin. Anders als erwartet, hängt diese Form der Zielorientierung am Ende der Grundschulzeit zum einen moderat positiv mit Lernzielen zusammen, zu anderen jedoch (noch) nicht mit Merkmalen eines ungünstig verlaufenden Lernprozesses (z.B. einem niedrigen Fähigkeitsselbstkonzept, einer niedrigen intrinsischen Motivation, einer erhöhten Leistungsangst). Die Ergebnisse deuten stattdessen vielmehr darauf hin, dass Annäherungsleistungsziele Ausdruck eines dysfunktionalen lernbezogenen Verhaltensmusters sind. So kann der positive Zusammenhang dieser mit der Leistungsangst als ein Indikator dafür interpretiert werden. Zudem zeigten Annäherungsleistungsziele einen praktisch bedeutsamen negativen Zusammenhang zu schulischen Leistungen. Hierbei handelt es sich um einen unerwarteten Befund, da Annäherungsleistungsziele in bisherigen Untersuchungen zumindest begrenzt positiv mit Schulleistungen zusammenhingen (siehe Metaanalyse von Hulleman et al., 2010). Die Lern- und Vermeidungsleistungsziele zeigten hingegen keinen praktisch bedeutsamen Zusammenhang zu den Noten bzw. Testleistungen in Studie 1, wobei die nicht signifikante Beziehung zwischen Vermeidungsleistungszielen und Schulleistungen in der geringen Validität dieser Form der Zielorientierung begründet liegen kann.

Da Studie 1 in einigen Punkten - insbesondere was das Zusammenhangsmuster der Zielorientierungen mit den schulischen Leistungen der Schüler betrifft - unerwartete Ergebnisse lieferte, sollte die gleiche Fragestellung in einer zweiten Studie an einer anderen Stichprobe und mittels eines anderen Instruments zur Erfassung von Zielorientierungen analysiert werden. Dazu wurden Schüler kurze Zeit nach dem Grundschulübergang befragt. Insgesamt deuten die Ergebnisse der Interkorrelationen der Zielorientierungen und der Zusammenhänge mit den Schulleistungen in der zweiten Studie auf eine befriedigendere Konstruktvalidität beim Einsatz der SELLMO. So konnte in Studie 2 eine negative Verbindung zwischen Vermeidungsleistungszielen und Schulleistungen bzw. eine positive Verbindung zwischen Lernzielen und der Leseleistung belegt werden. Jedoch lässt sich nicht eindeutig klären, worauf die von Studie 1 abweichenden Befunde zurückzuführen sind. So könnten zum einen das eingesetzte Instrument (Hulleman et al., 2010), zum anderen die Stichprobe oder die Schulleistungstests die Unterschiede in den Ergebnissen hervorgerufen haben. Welche Kriterien ausschlaggebend waren, lässt sich in der vorliegenden Arbeit nicht eindeutig klären. Dazu bedarf es weiterer Untersuchungen. 
Auch in Studie 2 konnte ein negativer Zusammenhang zwischen Annäherungsleistungszielen und Schulleistungen - insbesondere für die Testleistungen in Lesen und Mathematik - nachgewiesen werden. Bei den Schulnoten zeigte sich dieser Zusammenhang nach Kontrolle der anderen Dimensionen der Zielorientierung und der kognitiven Grundfähigkeit nicht mehr. Somit konnte in beiden Studien ein negativer Zusammenhang zwischen Annäherungsleistungszielen und Schulleistungen belegt werden. Jedoch kann aufgrund des Querschnittdesigns der Studien nicht geklärt werden, wie die Richtung des Zusammenhangs dabei aussieht. Denkbar wäre zum einen, dass hohe Annäherungsleistungsziele niedrigere schulische Leistungen bedingen (z.B. Köller, 1998), beispielsweise vermittelt über die Nutzung von bestimmten Lernstrategien. So könnte die verstärkte Nutzung von Oberflächenstrategien bzw. die verringerte Nutzung von Tiefenverarbeitungsstrategien dazu geführt haben, dass es zu niedrigeren Schulleistungen annäherungsleistungszielorientierter Schüler gekommen ist. Zum anderen kann der unerwartete Zusammenhang auch Folge der Lernbiographie des Schülers sein (Brophy, 2005). In diesem Falle würde man allerdings einen positiven Zusammenhang zwischen Annäherungsleistungszielen und Schulleistungen erwarten, da leistungsstarke Schüler auch höhere Erfolgserwartungen haben könnten und somit annäherungsleistungszielorientierter sein könnten. Nach Erhalt einer guten Note und dem Erleben der Leistungsüberlegenheit gegenüber anderen, könnte das Bedürfnis nach der Demonstration der eigenen Kompetenzen vor anderen bestärkt werden. Hat man eine gute Note bekommen und war somit besser als seine Klassenkameraden, könnte dies dazu führen, weiterhin besser sein zu wollen als diese. Da die Ergebnisse der Studien 1 und 2 jedoch keinen Hinweis darauf lieferten und eher im Gegenteil ein negativer Zusammenhang zu Schulleistungen gefunden wurde, sprechen die Ergebnisse für die Annahme, dass höhere Annäherungsleistungsziele zu niedrigeren schulischen Leistungen geführt haben, vice versa. Somit scheint Annäherungsleistungszielen eine die Kompetenzentwicklung hemmende Rolle in der Grundschule zu zukommen. Ein weiterer Indikator dafür stellt der Zusammenhang zu negativen Emotionen (Leistungsangst) dar. Emotionen haben sich in einschlägigen Untersuchungen als vermittelnde Variable zwischen Zielorientierungen und Schulleistungen erwiesen (Pekrun et al., 2009) und auch in der vorliegenden Arbeit war der negative Effekt der Annäherungsleistungsziele partiell durch die Leistungsangst vermittelt.

Der sehr schwache und meist nicht signifikante Effekt der Lernziele auf die Schulleistungen fügt sich in die Befundlage bisheriger Untersuchungen an Schülern dieser Altersgruppe ein (Bong, 2009; Finsterwald, 2006). Auch hier könnte die Nutzung von Lernstrategien zur Erklärung der Befunde herangezogen werden. Möglicherweise setzen Schüler am Ende der 
Grundschulzeit bzw. zu Beginn der Sekundarstufe I vermehrt Oberflächenstrategien ein (Hübner et al., 2010), sodass sich die positive Wirkung eines effektiven Einsatzes von Tiefenverarbeitungsstrategien auf Schulleistungen noch nicht zeigen kann. Eine andere Möglichkeit wäre, dass sich Lernziele erst in herausfordernderen Lernumwelten, wie sie es zumeist auf der weiterführenden Schule sind, positiv auf schulische Leistungen auswirken (Shim et al., 2008). Eine höhere Herausforderung verlangt wiederum ein höheres Maß an Eigenverantwortung ab, was dazu führen könnte, dass sich die positive Wirkung von Lernzielen erst unter diesen Bedingungen zeigt.

Da das Untersuchungsdesign der Studien 1 und 2 die Erfassung nicht nur der Ziffernnoten, sondern darüber hinaus auch curricular valider Testleistungen erlaubte, stellte eine zu untersuchende Frage die differenziellen Befunde bei der Verwendung von Noten und Testleistungen dar. In vielen bisherigen Untersuchungen zu Zielorientierungen wurden Ziffernnoten anstelle von curricular validen Testleistungen erfasst. Noten gelten jedoch als weniger objektiv (Südkamp \& Möller, 2009), da in die Notenvergabe über die Leistungen der Schüler hinaus weitere Aspekte (z.B. Motivation, Arbeits- und Lernverhalten) einfließen. In der vorliegenden Arbeit war es jedoch möglich, die unterschiedliche Bewertungspraxis zu berücksichtigen und Ergebnisse von Noten und Testleistungen an einer für die vierte Klassenstufe für die Bundesrepublik repräsentativen Stichprobe zu vergleichen. Die Befunde unterschieden sich nur gering zwischen Noten und Testleistungen, was allerdings nicht als ein Zeichen gleicher Zusammenhangsmuster zu den verschiedenen Formen von Zielorientierungen gedeutet werden sollte, da leider nur die Ergebnisse von Studie 1 verwendet werden konnten. Um genauere Aussagen über mögliche differenzielle Befunde bei der Verwendung von Noten und Testleistungen zu machen, bedarf es weiterer Forschung.

Die Ergebnisse der ersten beiden Studien belegen insgesamt, dass sich Zielorientierungen am Grundschulübergang faktoriell valide und reliabel messen lassen und dass Annäherungsleistungszielen am Grundschulübergang eine leistungshemmende Wirkung zukommt. Insgesamt deuten die Ergebnisse darauf hin, dass die lernpsychologische Bedeutung von Zielorientierungen bei Schülern dieser Altersgruppe teilweise anders erscheint als bei älteren Schülern oder Erwachsenen. 
(2) Zielorientierungen am Übergang zu verschiedenen Schulformen

Viele bisherige Studien, welche Zielorientierungen am Übergang von der Grundschule zur weiterführenden Schule untersucht haben, wurden oftmals im anglo-amerikanischen Raum durchgeführt. Da das Schulsystem dort allerdings nicht wie das bundesdeutsche Schulsystem gegliedert ist, war es in diesen Untersuchungen nicht möglich, Zielorientierungen an verschiedenen Schulformen zu analysieren. In der vorliegenden Arbeit konnte dieses Forschungsdesiderat behoben werden. Somit wurde die Entwicklung von Zielorientierungen erstmals an einer (für die vierte Klassenstufe) für die Bundesrepublik repräsentativen Stichprobe am Übergang zu verschiedenen Schulformen untersucht. Da sich die Lernumwelten an den verschiedenen Schulformen zum Teil deutlich voneinander unterscheiden und insbesondere das Gymnasium als kognitiv aktivierender und in einigen Bereichen herausfordernder als andere Schulformen beschrieben werden kann, stellte sich die Frage, ob differenzielle Entwicklungsmuster von Zielorientierungen an den verschiedenen Schulformen zu beobachten sind. Dazu wurden Schüler, welche auf ein Gymnasium wechselten, mit Schülern, welche auf eine der anderen Schulformen wechselten, verglichen (Kapitel 5, Studie 3).

Die Ergebnisse von Studie 3 belegen ein Absinken aller drei Formen von Zielorientierungen von Klassenstufe vier bis sechs unabhängig von der Schulform. Es ergaben sich lediglich geringe Mittelwertunterschiede zwischen Gymnasium und den anderen Schulformen. So waren Schüler, welche auf ein Gymnasium wechselten im Vergleich zu den anderen Schülern etwas weniger leistungszielorientiert zu allen drei Messzeitpunkten und etwas mehr lernzielorientiert am Ende der Grundschulzeit. Auch Köller (1998) kam in seiner Untersuchung von Zielorientierungen zu dem Ergebnis, dass Gymnasiasten weniger leistungszielorientiert sind als Schüler der anderen Schulformen. Allerdings untersuchte Köller Schüler der siebten Klassenstufe, was die Vergleichbarkeit der Ergebnisse erschwert.

Die Entwicklung der einzelnen Zielorientierungen wurde mit Hilfe latenter Wachstumskurvenmodelle untersucht. Dabei war es aufgrund der freien Schätzung der Zeitfunktion möglich, Aussagen über das relative Ausmaß der Veränderung innerhalb der definierten Zeitintervalle zu treffen. Ein zentrales Ergebnis der Untersuchung war, dass ein Großteil der Abnahme der Leistungsziele und hier insbesondere der Annäherungsleistungsziele (73,3\% der Abnahme zeigte sich zwischen Messzeitpunkt 1 und 2) am Grundschulübergang, also zwischen Klassenstufe vier und fünf stattfand. Eine vorsichtige Interpretation der Ergebnisse wäre, dass der Übergang von der Grundschule auf die weiterführende Schule bzw. die damit verbundenen Veränderungen die Entwicklung der Leistungsziele beeinflusst haben könnten. 
Es könnte argumentiert werden, dass sich der neue Kontext der weiterführenden Schule bereits nach kurzer Zeit vor allem auf solche leistungsthematischen Merkmale auswirkt, bei welchen soziale Vergleiche von großer Bedeutung sind. Da soziale Vergleichsprozesse insbesondere bei Leistungszielen eine wichtige Rolle spielen, könnte sich die veränderte Lernumwelt besonders schnell auf die Leistungsziele ausgewirkt haben. Bei Lernzielen zeigte sich hingegen eine weitaus geringere prozentuale Abnahme am Grundschulübergang (27,8 \%). Dies könnte darauf zurückgeführt werden, dass der intraindividuelle Vergleich der eigenen Leistungen bei lernzielorientierten Schülern von großer Bedeutung ist, sie sich demnach weniger mit den Leistungen ihrer Mitschüler vergleichen und sich die veränderte Lernumwelt weniger schnell auf die motivationale Ausrichtung dieser Schüler auswirkt. So konnten Studien zeigen, dass sich der Effekt des Übergangs in eine neue Schulform schneller auf das Fähigkeitsselbstkonzept auswirkt als beispielsweise auf die intrinsische Motivation der Schüler (Paulick et al., in preparation; Watermann et al., 2010).

Die Ergebnisse der latenten Wachstumskurvenmodelle belegten, dass sich das Entwicklungsmuster der jeweiligen Zielorientierungen nicht zwischen den einzelnen Schulformen unterscheidet. Somit scheint weniger die Schulform im Speziellen als vielmehr die weiterführende Schule im Generellen das Absinken aller drei Formen von Zielorientierungen beeinflusst zu haben. Die Ergebnisse von Studie 3 könnten demnach ein Indikator für die Vermutung sein, dass die Sekundarschule I eine Quelle der Motivationsabnahme und somit des Absinkens von Zielorientierungen als Teil der Motivation darstellt (Anderman \& Anderman, 1999; Anderman \& Midgley, 1997). Denkbar wäre, dass intrapersonale Veränderungen, welche mit der beginnenden Pubertät verbunden sind, das Absinken der Zielorientierungen bewirkt haben könnten. So verändern sich die Bedürfnisse der Schüler mit zunehmendem Alter. Das Bedürfnis nach Selbstbestimmung in Form von Autonomie, Kompetenzerleben und sozialer Eingebundenheit steigt (self-determination theory, Deci \& Ryan, 1985), wohingegen die Lernumwelt an der weiterführenden Schule diesen Bedürfnissen entgegensteht und oftmals Ausdruck gegensätzlicher Merkmale ist. Die Autonomie der Schüler wird durch ein höheres Maß an Strukturierung, Kontrolle, Regeln und Disziplin eingegrenzt. Der Wunsch nach Kompetenzerleben wird durch gesteigerte Leistungsanforderungen eingedämpft und die soziale Eingebundenheit der Schüler steht dem formellen und unpersönlicheren Schulklima an der weiterführenden Schule gegenüber. Dadurch kann es zu einer fehlenden Passung zwischen den veränderten Bedürfnissen der Schüler und der veränderten Lernumwelt der weiterführenden Schule gekommen sein, was wiederum zu einem Absinken der Motivation bzw. der Zielorientierungen geführt haben könnte (stage-environment-fit; Eccles et al., 1993). Da die 
Zielorientierungen sowohl am Gymnasium als auch an den anderen Schulformen abgesunken sind, scheint die Lernumwelt der Sekundarstufe I unabhängig von der besuchten Schulform mit den veränderten Schülerbedürfnissen zu kollidieren. Welche Merkmale des Lernkontextes im Speziellen zum Absinken der Zielorientierungen geführt haben, kann in der vorliegenden Arbeit jedoch nicht geklärt werden. Zur Beantwortung dieser Frage wäre ein Vergleich von Schülern des vierjährigen Grundschulsystems mit Schülern der sechsjährigen Grundschule vonnöten. Der Bedarf an zukünftiger Forschung dazu ist demnach nach wie vor gegeben.

Die Ergebnisse zur Entwicklung von Zielorientierungen fügen sich in die Reihe der Ergebnisse anderer Studien, welche die überarbeitete Version der PALS verwendeten (Bong, 2009; Finsterwald, 2006; Shim et al., 2008). Auch in diesen Untersuchungen konnte ein generelles Absinken aller Formen von Zielorientierungen nachgewiesen werden. Dies reiht sich in die Befundlage der von Hulleman et al. (2010) durchgeführten Metaanalyse ein und könnte als ein Zeichen dafür gedeutet werden, dass das Entwicklungsmuster ein für das eingesetzte Instrument typisches Muster darstellen könnte. Hulleman et al. zeigten, dass das jeweilige Instrument zur Erfassung der Zielorientierungen einen entscheidenden Einfluss auf die Ergebnisse nimmt. Inwieweit dies der Fall in der vorliegenden Untersuchung war, wäre in zukünftigen Untersuchungen zu prüfen.

Zusätzlich zur Entwicklung von Zielorientierungen war auch der Zusammenhang dieser mit schulischen Leistungen im Längsschnitt von Klassenstufe vier bis sechs Teil der vorliegenden Arbeit. So konnte zum einen das Zusammenhangsmuster mit Schulleistungen in der Grundschule und zum anderen auf den verschiedenen Schulformen der weiterführenden Schule (Gymnasium vs. andere Schulformen) betrachtet werden und mögliche schulformspezifische Unterschiede identifiziert werden. Ergebnis dabei war, dass sich Lernziele sowohl in der Grundschule als auch nach dem Übergang zu den verschiedenen Schulformen der Sekundarstufe I als leistungsförderlich erwiesen. Je höher die Lernzielorientierung der Schüler, umso besser ihre Schulnoten. Es konnten keine schulformspezifischen Unterschiede nachgewiesen werden, was darauf hin deutet, dass sich Lernziele unabhängig von der besuchten Schulform positiv auf schulische Leistungen auswirken. Die Unterschiede in den Lernumwelten der verschiedenen Schulformen scheinen dabei keine bedeutsame Rolle zu spielen. Die Vermutung, dass sich Lernziele insbesondere in kognitiv herausfordernden Lernumgebungen, wie dies am Gymnasium der Fall ist, positiv auswirken (Shim et al., 2008), konnte somit nicht bestätigt werden.

Weiterhin zeigten die Ergebnisse, dass sich Annäherungsleistungsziele nicht nur vor dem Grundschulübergang, sondern auch danach, zumindest am Gymnasium, negativ auf die 
Leistungsentwicklung auswirkten. Je höher die Annäherungsleistungsziele der Schüler, umso negativer die Notenentwicklung. Entgegen den Erwartungen verlor diese Form der Zielorientierung nach dem Grundschulübergang zum Gymnasium nicht ihre leistungshemmende Wirkung. In einer Lernumwelt, welche zum Teil als leistungsorientierter charakterisiert werden kann, sind Zielorientierungen, bei denen soziale Vergleiche und das Demonstrieren eigener Kompetenzen im Vordergrund stehen, besonders maladaptiv in Bezug auf schulische Leistungen. Wie auch schon von Middleton et al. (2004) vermutet, hängen Annäherungsleistungsziele negativ mit Schulleistungen bei sehr jungen Schülern zusammen. Wie die Richtung dabei aussieht, kann nicht eindeutig geklärt werden, da die jeweiligen Variablen kurze Zeit nacheinander erhoben wurden. Insgesamt kann allerdings bestätigt werden, dass Annäherungsleistungsziele mit niedrigeren schulischen Leistungen in der Grundschule bzw. zu Beginn der Sekundarstufe I zusammenhängen (Midgley et al., 2001) und dass dies insbesondere in kognitiv aktivierenden Lernumwelten mit hohen Leistungsstandards (Gymnasium) der Fall ist.

Auch hier kann der Einsatz von Lernstrategien zur Erklärung der Ergebnisse herangezogen werden. Leistungsziele wurden oftmals mit der Nutzung von Oberflächenstrategien in Verbindung gebracht (z.B. Bouffard et al., 1995; Midgley \& Urdan, 2001; Nolen, 1988; Köller, 1998; Pintrich \& De Groot, 1990). Am Gymnasium könnte die Nutzung dieser Strategien jedoch besonders leistungshemmend sein, da die hohen Leistungsanforderungen und Leistungsstandards die Nutzung von Tiefenverarbeitungsstrategien erfordern könnten. Oberflächenstrategien könnten sich somit am Gymnasium möglicherweise besonders negativ in Bezug auf schulische Leistungen auswirken.

Eine weitere mögliche mediierende Variable zwischen Annäherungsleistungszielen und Schulleistungen könnten Emotionen sein. So haben bisherige Untersuchungen zeigen können, dass Leistungsziele vermehrt mit negativen Emotionen wie beispielsweise Angst oder Sorgen in Verbindung stehen. Negative Emotionen wirken sich wiederum negativ auf schulische Leistungen aus (Pekrun et al., 2002). Somit könnte - vermittelt über bestimmte Emotionen - der Zusammenhang zwischen Annäherungsleistungszielen und niedrigen schulischen Leistungen erklärt werden. Tatsächlich deuten die Ergebnisse aus Studie 1 darauf hin: Annäherungsleistungsziele hingen am Ende der Grundschulzeit positiv mit Leistungsangst zusammen.

Auch wenn die gefundenen Effekte von kleiner Größenordnung waren, können sie als bedeutend interpretiert werden, da die Befunde stets unter Kontrolle der anderen Dimensionen 
der Zielorientierungen, der kognitiven Grundfähigkeit und des Geschlechts der Schüler erbracht wurden und unter diesen Konditionen auch kleine Effekt von praktischer Relevanz sind.

Insgesamt deuten die Ergebnisse von Studie 3 darauf hin, dass sich zum einen Lernziele positiv auf die Notenentwicklung nicht nur vor sondern auch nach dem Grundschulübergang auswirken und dass zum anderen Annäherungsleistungszielen eine leistungshemmende Wirkung in der Grundschule bzw. auch nach dem Übergang auf ein Gymnasium zukommt.

(3) Untersuchung von Bezugsgruppeneffekten

Die vorliegende Arbeit beschäftigte sich mit Zielorientierungen in der Grundschule bzw. der Entwicklung dieser am Grundschulübergang zu den verschiedenen Schulformen und dem Zusammenhang dieser mit schulischen Leistungen im Längsschnitt und nahm zusätzlich die Untersuchung von Bezugsgruppeneffekten auf Zielorientierungen in den Fokus (Studie 4). Studien zu Kontrast- bzw. Assimilationseffekten bezogen sich bislang ausschließlich u.a. auf das Fähigkeitsselbstkonzept, das Interesse oder die intrinsische Motivation der Schüler (z.B. Marsh et al., 2001; Trautwein, Lüdtke, Marsh et al., 2006). Seit geraumer Zeit wurde allerdings die Forderung nach einer Ausweitung der Forschung zu Bezugsgruppeneffekten auch auf andere Merkmale schulischen Lernens immer lauter. In Studie 4 wurde dieser Forderung zu diesem, insbesondere für die Übergangsforschung, relevanten Thema nachgegangen und eine Ausweitung der Untersuchung von Bezugsgruppeneffekten auf Zielorientierungen vorgenommen. Da Zielorientierungen einen Bestandteil der Motivation darstellen und bei ihnen soziale Vergleichsprozesse eine wichtige Rolle spielen, wurde davon ausgegangen, dass sich ein Bezugsgruppenwechsel ähnlich wie auf Selbstkonzept oder intrinsische Motivation der Schüler auch auf Zielorientierungen auswirken könnte. Dazu wurden in einem ersten Schritt die in der Studie von Watermann et al. (2010) nachgewiesenen Kontrasteffekte auf das Fähigkeitsselbstkonzept und die intrinsische Motivation nach dem Übergang auf das Gymnasium repliziert. Schüler, welche nach dem Grundschulübergang auf ein Gymnasium wechselten, hatten zum einen ein höheres Selbstkonzept und eine höhere intrinsische Motivation vor dem Übergang, zum anderen sanken ihr Selbstkonzept und ihre Motivation nach dem Übergang weitaus stärker als dies bei den anderen Schülern der Fall war. In einem zweiten Schritt wurden die Analysen der konditionalen Wachstumskurvenmodelle auch auf die Zielorientierungen ausgeweitet. Die Ergebnisse zeigten, dass Lernziele bei zukünftigen Gymnasiasten stärker ausgeprägt waren als bei den anderen Schülern und dass die Schulform einen signifikanten Effekt auf die Entwicklung der Lernziele nach dem Übergang auf die weiterführende Schule 
hat. Dieser Effekt verschwand allerdings nach Kontrolle der Schulnoten in Klassenstufe fünf. Tendenziell deuteten die Befunde darauf hin, dass Lernziele bei Gymnasiasten etwas stärker abnehmen als bei Schülern, die auf eine andere Schulform wechseln. Insgesamt sanken die Lernziele jedoch an allen Schulformen.

Bei den beiden Facetten der Leistungsziele, den Annäherungs- und Vermeidungsleistungszielen, zeigten sich keine Bezugsgruppeneffekte nach dem Wechsel auf die weiterführende Schule. Stattdessen konnte ein Absinken der Leistungsziele an allen Schulformen in gleicher Weise belegt werden. Diese Befunde deuten darauf hin, dass nicht die Schulform an sich die Entwicklung der Zielorientierungen beeinflusst hat, sondern dass die Lernumwelt der Sekundarstufe I generell das Absinken der Zielorientierungen verursacht haben könnte. Wie auch schon im vorangegangenen Abschnitt (2) diskutiert wurde, könnte eine fehlende Passung zwischen den veränderten Schülerbedürfnissen und der neuen Lernumwelt der weiterführenden Schule dafür verantwortlich sein, dass die Motivation bzw. die Zielorientierungen der Schüler abnahmen (stage-environment fit theory; Eccles et al., 1993).

Eine weitere Forschungsfrage, welche sich im Zusammenhang mit dem BFLPE stellte, bezog sich auf einen möglichen mediierenden Effekt von Schulnoten. Im Gegensatz zu einigen anderen Studien zum BFLPE wurde in der vorliegenden Arbeit nicht nur der Effekt von Testleistungen betrachtet, sondern auch der Effekt von Schulnoten. Trautwein, Lüdtke, Marsh et al. (2006) wiesen nach, dass Schulnoten die schulformspezifischen Unterschiede im Fähigkeitsselbstkonzept und der Motivation der Schüler erklären können. So waren in ihrer Untersuchung die Noten von Gymnasiasten besser als die von Haupt- bzw. Realschülern. Gymnasiasten hatten wiederum ein höheres Fähigkeitsselbstkonzept, was als eine Folge der besseren Noten gedeutet werden kann. Tatsächlich verschwand der Effekt der Schulform auf das Selbstkonzept nach Kontrolle der Schulnoten in der Studie von Trautwein, Lüdtke, Marsh et al. (2006). Auch in der vorliegenden Arbeit sollte der Effekt der Schulnoten auf den BFLPE untersucht werden. Dazu wurden nicht nur die schulischen Leistungen der Schüler in Form von Testleistungen und Ziffernnoten vor dem Übergang in die Analysen bezogen, sondern in einem weiteren Schritt auch die Noten nach dem Übergang zu den verschiedenen Schulformen der Sekundarstufe I. Die Ergebnisse der Analysen belegten zwar eine deutliche Abnahme des Bezugsgruppeneffekts auf das Fähigkeitsselbstkonzept der Schüler, jedoch blieb der BFLPE weiterhin signifikant und bedeutsam. Somit kann davon ausgegangen werden, dass der Effekt der Bezugsgruppe auf das Absinken des Selbstkonzepts bei Gymnasiasten zum Teil durch die Notenvergabe vermittelt wurde. Die Größe des BFLPEs auf die intrinsische Motivation veränderte sich nach Kontrolle der Schulnoten in Klassenstufe fünf hingegen nicht be- 
deutend. Auch bei vergleichbarer Schulleistung der Schüler an der weiterführenden Schule beeinflusst die Schulform die Entwicklung der Motivation: die Motivation von Gymnasiasten sank stärker als die der anderen Schüler. Demnach scheint die unterschiedliche Bewertungspraxis bei der Notenvergabe an den jeweiligen Schulformen keinen Einfluss auf die Motivationsentwicklung der Schüler genommen zu haben. Bei den Lernzielen zeigte sich hingegen, dass der negative Bezugsgruppeneffekt auf die Entwicklung der Lernziele nach Kontrolle der Schulnoten in Klassenstufe fünf nicht mehr signifikant war. Somit wurde dieser Effekt über die Schulnoten nach dem Übergang bzw. über die unterschiedliche Bewertungspraxis bei der Notenvergabe an den jeweiligen Schulformen vermittelt.

Insgesamt deuten die Ergebnisse von Studie 4 darauf hin, dass sich der Wechsel der Bezugsgruppe größtenteils nicht in der gleichen Form auf die Entwicklung der Zielorientierungen auswirkt wie es beim Fähigkeitsselbstkonzept und der intrinsischen Motivation der Fall ist. Somit scheinen sich Zielorientierungen konzeptuell vom Selbstkonzept und insbesondere von der intrinsischen Motivation zu unterscheiden, denn anders als vermutet, konnte kein - bzw. bei Lernzielen nur ein kleiner - BFLPE auf Zielorientierungen am Gymnasium nachgewiesen werden. Des Weiteren zeigten die Analysen, dass Zielorientierungen weniger durch Schulnoten beeinflusst wurden als Selbstkonzept oder Motivation. Stattdessen scheinen andere Faktoren eine bedeutsamere Rolle zu spielen. Da in der vorliegenden Arbeit nicht eindeutig geklärt werden kann, welche Faktoren die Entwicklung von Zielorientierung beeinflussen, wäre die Beantwortung dieser Frage Aufgabe zukünftiger Forschung. 


\subsection{Grenzen der Arbeit und Ansätze für die weitere Forschung}

Abschließend soll auf einige Grenzen der vorliegenden Arbeit hingewiesen werden. Zum einen erfolgte die Erfassung der Zielorientierungen bereichsübergreifend. Die Zielorientierungen wurden also gemeinsam für die einzelnen Schulfächer erhoben, sodass nicht ausgeschlossen werden kann, dass keine schulfachspezifischen Unterschiede zum einen in der Ausprägung der jeweiligen Zielorientierungen und zum anderen im Zusammenhangsmuster zwischen Zielorientierungen und schulischen Leistungen bestehen. Betrachtet man die bisherige Forschung zur Bereichsspezifität bzw. -generalität von Zielorientierungen, so ist die Forschungslage eher inkonsistent. Duda und Nicholls (1992) fanden in ihrer Studie, in welcher sie Zielorientierungen getrennt für Sport und die übrigen Schulfächer erhoben haben, ein klares Indiz für die Generalität von Zielorientierungen. Die Lernzielorientierung korrelierte mit $r$ $=.67$ zwischen beiden Domänen und die Leistungszielorientierung mit $r=.62$. Die anschließende Faktorenanalyse ergab zwar eine klare Trennung der Zielorientierungen, allerdings keine Differenzierung der Domänen. Ähnliche Ergebnisse fanden Stipek und Gralinski (1996) für die Klassenstufen drei bis sechs. In ihrer längsschnittlichen Untersuchung wurden Zielorientierungen für die Schulfächer Mathematik und Sozialunterricht erhoben. Die Korrelation je nach Messzeitpunkt zwischen den Lernzielen der Domänen lag bei $r=.62 / .69$ und zwischen den Leistungszielen bei $r=.69 / .79$. Die Faktorenanalyse ergab keine Trennung der Domänen, aber der Zielorientierungen. Auch die Untersuchung von Meece et al. (1988) hat eine hohe Konsistenz der Zielorientierungen über sechs verschiedene naturwissenschaftliche Lernbereiche nachgewiesen. Die Korrelationen der einzelnen Zielorientierungen lagen zwischen $r=.44$ und $r=.83$, woraus die Autoren resümierten: „The results indicate a reasonable degree of consistency and stabillity in children's gaol orientation (...) across the six learning acticities“" (Meece et al., 1988, S. 518). Hingegen zeigten neuere Studien (Bong, 2001; Sparfeldt et al., 2007) tendenziell eher eine Generalität von Leistungszielen und eine Bereichsspezifität von Lernzielen. Inwieweit dies auf Zielorientierungen am Grundschulübergang zutrifft, kann in der vorliegenden Arbeit nicht geklärt werden und könnte somit Gegenstand zukünftiger Forschung sein.

Des Weiteren muss an dieser Stelle angemerkt werden, dass die vorliegende Arbeit zwar Aufschluss über die Entwicklung von Zielorientierungen und deren Zusammenhang zu schulischen Leistungen am Grundschulübergang gibt, es allerdings nicht geklärt werden kann, worin die Veränderungen in den Zielorientierungen begründet liegen, ob diese übergangsoder aber entwicklungsbedingt sind. Um diese Frage befriedigend zu klären, wäre es notwen- 
dig, die Schüler der Übergangsstudie mit Schülern aus Bundesländern, in denen der Grundschulübergang erst nach Klassenstufe sechs stattfindet, zu vergleichen. Erst wenn die Gründe für das Absinken der Zielorientierungen am Grundschulübergang und mögliche vermittelnde Variablen identifiziert wurden, kann über praktische Implikationen nachgedacht werden. Aus diesem Grund können an dieser Stelle lediglich vorsichtige Überlegungen über eventuelle Implikationen für die Schulpraxis angestellt werden. So könnte beispielsweise einer möglichen fehlenden Passung zwischen Schülerbedürfnissen und den jeweiligen Lernumwelten der verschiedenen Schulformen der Sekundarstufe I entgegengewirkt werden, indem vermehrt auf die Bedürfnisse der Schüler nach Autonomie, Kompetenzerleben und sozialer Eingebundenheit eingegangen wird. Zudem wäre eine Förderung von Lernzielen sowohl vor als auch nach dem Grundschulübergang erstrebenswert, da sich diese auch in der vorliegenden Arbeit als leistungsförderlich erwiesen haben. Demgegenüber sollte die Lernumwelt der Sekundarstufe I bewusst einen (annäherungs-)leistungszielorientierten Fokus vermeiden, da sich insbesondere diese Form der Zielorientierung als leistungshemmend bei Schülern dieser Altersgruppe erwiesen hat.

Eine Stärke der vorliegenden Arbeit stellt die längsschnittliche Erfassung von Zielorientierungen am Grundschulübergang dar, jedoch wäre zum einen eine weitere Verfolgung der Schüler in den verschiedenen Schulformen interessant, um den Einfluss der Schulformen auf die Entwicklung der Zielorientierungen und deren Zusammenhang zu Schulleistungen auch zu einem späteren Zeitpunkt zu untersuchen und Implikationen abzuleiten. Zum anderen wäre eine mikrogenetische Betrachtung der Entwicklung von Zielorientierungen am Grundschulübergang ein interessanter Ansatz für zukünftige Forschung, um eventuelle Veränderungen, welche sehr kurze Zeit nach dem Schulwechsel stattgefunden haben, zu erfassen. Da die Abstände zwischen den Erhebungszeitpunkten relativ groß waren (ca. sechs Monate) kann nicht ausgeschlossen werden, dass sich die Befunde zwischen den Messzeitpunkten nicht von den in der vorliegenden Arbeit dargestellten Ergebnissen unterschieden hätten. Interessant wäre auch die qualitative Untersuchung von Zielorientierungen sowohl vor als auch nach dem Grundschulübergang, um so mögliche Einflussfaktoren für die Entwicklung von Zielorientierungen am Übergang und für die Bewältigung dessen zu identifizieren. 


\section{Zusammenfassung}

Zielorientierungen stellen einen großen Theoriekomplex im Bereich der Motivationsforschung dar, welchem in den letzten Jahrzehnten viel Aufmerksamkeit geschenkt wurde. Dabei zeigten Zielorientierungen häufig eine Verbindung zu verschiedenen motivationalen, emotionalen und kognitiven Merkmalen des schulischen Lernens (siehe Metanalyse von Hulleman et al., 2010). Ein Forschungsdesiderat in diesem Zusammenhang stellte jedoch die Untersuchung von Zielorientierungen an Schülern der Primarstufe dar. Die bis dato bekannten Studien fanden entweder nicht im deutschsprachigen Raum statt, bezogen sich lediglich auf eine kleine, homogene und nicht repräsentative Stichprobe oder nahmen eine domänenspezifische Betrachtung von Zielorientierungen vor. Das zentrale Ziel der vorliegenden Arbeit war es hingegen, Zielorientierungen an einer- im Falle der Primarstufe für die Bundesrepublik repräsentativen Stichprobe in einer für die zukünftige Laufbahn der Schüler sehr wichtigen Phase des Grundschulübergangs zu untersuchen. Dabei stellte sich zuallererst die Frage, ob sich Zielorientierungen bereits bei dieser Altersgruppe reliabel und valide messen lassen und in welchem Zusammenhang Zielorientierungen mit Schulleistungen am Ende der Primarschule stehen.

Der Übergang von der Grundschule auf die weiterführende Schule stellt für jeden Schüler ein bedeutsames und kritisches Lebensereignis dar, da es mit zahlreichen Veränderungen auf verschiedenen Ebenen einhergeht. Im internationalen Vergleich stellt dabei das bundesdeutsche Schulsystem mit seiner Dreigliedrigkeit eine Besonderheit dar. Mit dem Übergang auf eine der verschiedenen Schulformen und der damit verbundenen Leistungsdifferenzierung werden Schüler schon sehr früh selektiert und ihr Lebensweg damit in bedeutsamer Weise vorbestimmt. Im Zusammenhang mit dem Grundschulübergang wurden bereits zahlreiche Phänomene des schulischen Lernens untersucht, welche sich beispielsweise auf die Bewältigung des Übergangs bezogen (z.B. Finsterwald, 2006; Sirsch, 2000) oder auf die Entwicklung von verschiedenen motivationalen Schülermerkmalen (z.B. Aust et al, 2010; Valtin \& Wagner, 2004). In keiner bisherigen Untersuchung wurde allerdings der Entwicklung von Zielorientierungen beim Übergang auf die Sekundarstufe I bzw. die damit verbundenen potenziellen schulformspezifischen Unterschiede an einer repräsentativen Stichprobe betrachtet. Des Weiteren war in der vorliegenden Arbeit der Zusammenhang zwischen Zielorientierungen und schulischen Leistungen nicht nur am Ende der Grundschulzeit, sondern auch nach dem Grundschulübergang von großem Interesse. Dabei stellte sich zum einen die Frage, in welchem Zusammenhang Zielorientierungen mit Schulleistungen stehen und ob sich das Zu- 
sammenhangsmuster am Übergang von der Grundschule auf die weiterführende Schule verändert. Zum anderen sollten mögliche Unterschiede zwischen den Schulformen identifiziert werden.

Ein weiteres Forschungsdesiderat, welches die vorliegende Arbeit behob, war die Betrachtung von Bezugsgruppeneffekten bei Zielorientierungen. In der bisherigen Forschung zu den Effekten eines Bezugsgruppenwechsels wurde sich ausschließlich auf Schülermerkmale wie Fähigkeitsselbstkonzept, intrinsische Motivation, Interesse oder auch Bildungsaspirationen bezogen (z.B. Marsh et al., 2001; Trautwein, Lüdtke, Marsh et al., 2006). Hingegen stellte die Untersuchung von Kontrast- bzw. Assimilationseffekten auf Zielorientierungen eine Forschungslücke dar. Da soziale Vergleichsprozesse eine nicht unbedeutende Rolle auch bei Zielorientierungen spielen und in zahlreichen Untersuchungen ein Zusammenhang zwischen Zielorientierungen und beispielsweise Selbstkonzept oder Interesse nachgewiesen werden konnte, wurde in der vorliegenden Arbeit vermutet, dass sich auch bei Zielorientierungen Bezugsgruppeneffekte zeigen könnten.

Zur Beantwortung der verschiedenen Forschungsfragen wurden die Daten der Übergangsstudie analysiert, welche eine Erweiterung der TIMS-Studie 2007 darstellt. Dabei handelte es sich um eine groß angelegte Längsschnittstudie über vier Messzeitpunkte (einen vor und drei Messzeitpunkte nach dem Übergang), wobei sich in der vorliegenden Arbeit auf drei Erhebungszeitpunkte bezogen wird (Ende Klassenstufe vier, Mitte Klassenstufe fünf und Anfang Klassenstufe sechs).

Um die Frage der Reliabilität und Konstruktvalidität von Zielorientierungen und deren Zusammenhang zu schulischen Leistungen in der Grundschule zu beantworten, wurden im ersten Teil der Untersuchung (Studie 1) die Schüler der Primarstufe (Ende Klassenstufe vier) befragt $(N=5242)$. Die Ergebnisse der konfirmatorischen Faktorenanalysen bestätigten dabei die theoretisch postulierte 3-Faktorstruktur mit den Faktoren Lern-, Annäherungsleistungsund Vermeidungsleistungsziele. Insgesamt konnten die Zielorientierungen am Ende der Grundschulzeit reliabel und zum großen Teil valide gemessen werden. Obwohl die Anzahl der Items (insbesondere bei den Vermeidungsleistungszielen) relativ gering war, erreichten die Reliabilitäten zufriedenstellende bis sehr gute Werte. Jedoch deuteten die Ergebnisse auf eine eingeschränkte Konstruktvalidität des eingesetzten Instruments zur Erfassung der Zielorientierungen (modifizierte Version der PALS) in Bezug auf Vermeidungsleistungsziele hin. So ergab sich ein unerwartet positiver Zusammenhang zu Lernzielen, bzw. Selbstkonzept und Motivation. Ein weiterer Befund von Studie 1 war, dass Annäherungsleistungsziele negativ mit schulischen Leistungen zusammenhingen. Da dieser Befund unerwartet kam, wurde in 
einer weiteren Studie (Studie 2) eine Replikation der Ergebnisse anhand einer anderen Stichprobe und mittels eines anderen Instruments zur Erfassung von Zielorientierungen (SELLMO) angestrebt. Dazu wurden die Daten von $N=294$ Schülern eines Gymnasiums und einer Integrierten Gesamtschule kurze Zeit nach dem Übergang analysiert. Auch in Studie 2 konnte ein negativer Zusammenhang zwischen Annäherungsleistungszielen und schulischen Leistungen gezeigt werden.

Insgesamt sprechen die Befunde aus Studien 1 und 2 dafür, dass sich Zielorientierungen am Grundschulübergang reliabel messen lassen. Allerdings geben die unerwarteten und zugleich eindrucksvollen Ergebnisse zu Annäherungsleistungszielen Hinweise darauf, dass die lernpsychologische Bedeutung dieser Konstrukte bei dieser Altersgruppe teilweise anders zu sein scheint als bei älteren Schülern oder Erwachsenen.

In Studie 3 stand zum einen die Entwicklung von Zielorientierungen von Klassenstufe 4 bis 6 und zum anderen der Zusammenhang derer zu Schulleistungen an den verschiedenen Schulformen im Vordergrund. Dazu wurden die längsschnittlichen Daten der Übergangsstudie ( $N=1646)$ analysiert und Schüler, die auf ein Gymnasium wechselten, mit Schülern, die auf eine der anderen Schulformen wechselten, verglichen. Das Ergebnis der Wachstumskurvenmodelle war eine generelle Abnahme aller drei Formen von Zielorientierungen von Klassenstufe vier bis sechs an allen Schulformen- das Entwicklungsmuster unterschied sich nicht zwischen den jeweiligen Schulformen. Es ergaben sich lediglich geringe Mittelwertdifferenzen: zukünftige Gymnasiasten waren etwas weniger leistungszielorientiert zu allen drei Messzeitpunkten und etwas mehr lernzielorientiert in Klassenstufe vier. Interessanterweise erfolgte die Abnahme der Leistungsziele deutlich schneller nach dem Grundschulübergang als die Abnahme der Lernziele, was dafür sprechen könnte, dass Leistungsziele möglicherweise sensibler auf Veränderungen in der Lernumwelt als Lernziele reagieren. Das Ergebnis der CrossLagged Panel Modelle war, dass - unter Kontrolle der anderen Dimensionen der Zielorientierungen, des Geschlechts und der kognitiven Grundfähigkeit - Lernziele sowohl vor als auch nach dem Grundschulübergang positiv mit schulischen Leistungen an allen Schulformen zusammenhingen. Annäherungsleistungsziele zeigten hingegen einen negativen Zusammenhang zu Schulnoten in Klassenstufe vier und in Klassenstufe fünf am Gymnasium.

Insgesamt sprechen die Befunde von Studie 3 dafür, dass Lernziele am Grundschulübergang leistungsförderlich sind, anders als Annäherungsleistungsziele, welchen hingegen eine leistungshemmende Wirkung zum einen am Ende der Grundschulzeit und zum anderen zu Beginn der Sekundarstufe I am Gymnasium zukommt. 
In Studie 4 ging es dann um den Effekt der Schulform auf die Entwicklung von Zielorientierungen. Diesbezüglich stellte sich zum einen die Frage, ob sich der oftmals für das Fähigkeitsselbstkonzept und die intrinsische Motivation nachgewiesene Big-Fish-Little-PondEffekt (BFLPE; Marsh, 1987) auch bei Zielorientierungen zeigt. Zum anderen sollte untersucht werden, inwieweit die Schulnoten nach dem Bezugsgruppenwechsel zusätzlich zu den schulischen Leistungen am Ende der Grundschulzeit den BFLPE erklären können. Ergebnis der konditionalen Wachstumskurvenmodelle war, dass sich der BFLPE auf Selbstkonzept und Motivation nach dem Wechsel auf ein Gymnasium zeigte, Selbstkonzept und Motivation am Gymnasium also stärker sanken als an den anderen Schulformen. Dieser Effekt ließ sich auch bei Lernzielen, jedoch nicht bei Leistungszielen nachweisen. Außerdem zeigte sich, dass der BFLPE auf Selbstkonzept und Lernziele der Schüler teilweise über die Schulnoten nach dem Grundschulübergang vermittelt wurde.

Insgesamt sprechen die Befunde von Studie 4 dafür, dass es sich beim Konstrukt der Zielorientierungen - insbesondere bei Leistungszielen - um ein sich deutlich vom Selbstkonzept und von der intrinsischen Motivation abgrenzbares Konstrukt handelt und der Wechsel der Bezugsgruppe keinen bedeutsamen Einfluss auf diese Zielorientierungen ausübt.

Als zentrales Ergebnis der vorliegenden Arbeit lässt sich festhalten, dass sich zum einen Zielorientierungen bereits bei sehr jungen Schülern - am Ende der Grundschulzeit reliabel und zum großen Teil valide messen lassen, der Zusammenhang zu schulischen Leistungen jedoch darauf hinweist, dass die Bedeutung des Konstrukts der Zielorientierungen bei Schülern am Grundschulübergang von der älterer Schüler oder auch Erwachsener unterscheidet. Die leistungshemmende Wirkung von Annäherungsleistungszielen soll dabei besonders herausgestellt werden. Hier sollte insbesondere in der pädagogischen Praxis das Bewusstsein für die Wirkung dieser geweckt werden um deren leistungshemmenden Wirkung möglichst entgegenzuwirken. 


\section{Literaturverzeichnis}

Ames, C. (1984). Achievement attributions and self-instructions in competitive and individualistic goal structures. Journal of Educational Psychology, 76, 478-487.

Ames, C. (1992). Classroom: Goals, structure and motivation. Journal of Educational Psychology, 84, 261-271.

Ames, C., \& Ames, R. (1984). Systems of student and teacher motivation: Toward a qualitative definition. Journal of Educational Psychology, 76, 535-556.

Ames, C., \& Archer, J. (1988). Achievement goals in the classroom: Students' learning strategies and motivation processes. Journal of Educational Psychology, 80, 260-270.

Anderman, E. M., Austin, C. C., \& Johnson, D. M. (2002). The development of goal orientation. In A. Wigfield, \& J. S. Eccles, (Eds.), The Development of achievement motivation. A volume in the educational psychology series (pp. 197-220). San Diego, CA: Academic Press.

Anderman, E. M., \& Maehr, M. L. (1994). Motivation and schooling in the middle grades. Review of Educational Research, 64(2), 287-309.

Anderman, E. M., Maehr, M. L., \& Midgley, C. (1999). Declining motivation after the transition to middle school: Schools can make a difference. Journal of Research and Development in Education, 32, 131-147.

Anderman, E. M., \& Midgley, C. (1997). Changes in achievement goal orientations, perceived academic competence, and grades across the transition to middle-level schools. Contemporary Educational Psychology, 22, 269-298.

Anderman, E. M., \& Midgley, C. (2004). Changes in self-reported academic cheating across the transition from middle school to high school. Contemporary Educational Psychology, 29, 499-517.

Anderman, L. H., \& Anderman, E. M. (1999). Social predictors of changes in students' achievement goal orientations. Contemporary Educational Psychology, 25, 21-37.

Aust, K., Watermann, R., \& Grube, D. (2009). Konsequenzen von Leistungsgruppierungen auf die Entwicklungsverläufe des allgemeinen und fachspezifischen Fähigkeitsselbstkonzepts nach dem Übergang in die Sekundarstufe. Zeitschrift für Erziehungswissenschaft, 12 (Sonderheft), 328-351.

Aust, K., Watermann, R., \& Grube, D. (2010). Selbstkonzeptentwicklung und Zielorientierungen nach dem Übergang von der Grundschule in die weiterführende Schule. Zeitschrift für Pädagogische Psychologie, 24(2), 95-109. 
Baeriswyl, F., Trautwein, U., Wandeler, C., \& Lüdtke, O. (2009). Testleistung, Noten, Übertrittsempfehlung oder Übertrittsgutachten: Vorhersagekraft unterschiedlicher Elemente von Übertrittsverfahren am Ende der Grundschule für die Schulleistung am Ende der Sekundarstufe I. Zeitschrift für Erziehungswissenschaft, 12 (Sonderheft) (S. 353-372). Wiesbaden: VS Verlag für Sozialwissenschaften.

Bandalos, D. L., Finney, S. J., \& Geske, J. A. (2003). A model of statistics performance based on achievement goal theory. Journal of Educational Psychology, 95, 604-616.

Barron, K. E., \& Harackiewicz, J. M. (2001). Achievement goals and optimal motivation: Testing multiple goal models. Journal of Personality and Social Psychology, 80, 706722 .

Baumert, J., Roeder, M. P., Gruehn, S., Heyn, S., Köller, O., \& Rimmele, R. et al. (1996). Bildungsverläufe und psychosoziale Entwicklung im Jugendalter (BIJU). In K.-P. Treumann, G. Neubauer, R. Möller \& J. Abel (Hrsg.), Methoden und Anwendungen empirischer pädagogischer Forschung (S. 170-180). Münster: Waxmann.

Baumert, J., Trautwein, U., \& Artelt, C. (2003). Schulumwelten- institutionelle Bedingungen des Lehrens und Lernens. In J. Baumert, C. Arteilt, E. Klieme, M. Neubrand, M. Prinzel, U. Schiefele, W. Schneider, K.-J. Tillmann \& M. Weiss (Hrsg.), PISA 2000. Ein differenzierter Blick auf die Länder der Bundesrepublik Deutschland (S. 261-331). Opladen: Leske+Budrich.

Becker, M., Gresch, C., Baumert, J., Watermann, R., Schnitger, D., \& Maaz, K. (2010). Durchführung, Daten und Methoden. In K. Maaz, J. Baumert, C. Gresch, N. McElvany (Hrsg.), Der Übergang von der Grundschule in die weiterführende SchuleLeistungsgerechtigkeit und regionale, soziale und ethnisch-kulturelle Disparitäten: Zusammenfassung der zentralen Befunde (S. 107-121). Bonn, Berlin: BMBF.

Beelmann, W. (2000). Entwicklungsrisiken und -chancen bei der Bewältigung normativer sozialer Übergänge im Kindesalter. In C. Leyendecker \& T. Horstmann (Hrsg.), Große Pläne für kleine Leute (S. 71-77). München: Ernst Reinhardt.

Bellenberg, G. (2005). Wege durch die Schule - Zum Zusammenhang zwischen institutionalisierten Bildungswegen und individuellen Bildungsverläufen im deutschen Schulsystem. In Bildungsforschung (Band 2),

URL:http://www.bildungsforschung.org/index.php/bildungsforschung/article/viewFile $/ 15 / 13$.

Berlyne, D. E. (1978). Curiosity and learning. Motivation and Emotion, 2, 97-175. 
Bollen, K. A., \& Curran, P. J. (2006). Latent curve models: A structural equation perspective. Hoboken, NJ: Wiley.

Bong, M. (2001). Between- and within- domain relations of academic motivation among middle and high school students: Self-efficacy, task-value and achievement goals. Journal of Educational Psychology, 1, 23-34.

Bong, M. (2009). Age-related differences in achievement goal differentiation. Journal of Educational Psychology, 101(4), 879-896.

Bonsen, M., Lintorf, K. A., Bos, W., \& Frey, K. (2008). TIMSS 2007: Mathematische und naturwissenschaftliche Kompetenzen von Grundschulkindern in Deutschland im internationalen Vergleich. In W. Bos, M. Bonsen, J. Baumert, M. Prenzel, C. Selter \& G. Walther (Hrsg.), TIMSS: Dokumentation der Erhebungsinstrumente zur Trends in International Mathematics and Science Study (S. 19-48). Münster: Waxmann.

Bos, W., Bonsen, M., Baumert, J., Prenzel, M., Selter, C., \& Walther, G. (Hrsg.). (2008). Mathematische und naturwissenschaftliche Kompetenz von Grundschülern in Deutschland im internationalen Vergleich. Münster: Waxmann.

Bos, W., \& Pietsch, M. (2005). KESS 4. Kompetenzen und Einstellungen von Schülerinnen und Schülern - Jahrgangsstufe 4. Hamburg: Behörde für Bildung und Sport.

Bos, W., Valtin, R., Lankes, E.-M., Schwippert, K., Voss, A., \& Badel, I. et al. (2004). Lesekompetenzen am Ende der vierten Jahrgangsstufe in einigen Ländern der Bundesrepublik Deutschland im nationalen und internationalen Vergleich. In W. Bos, E.-M. Lankes, M. Prenzel, K. Schwippert, R. Valtin \& G. Walther (Hrsg.), IGLU: Einige Länder der Bundesrepublik Deutschland im nationalen und internationalen Vergleich (S. 49-92). Münster: Waxmann.

Bouffard, T., Boisvert, J., Vezeau, C., \& Larouche, C. (1995). The impact of goal orientation on self-regulation and performance among college students. British Journal of Educational Psychology, 65, 317-329.

Brandtstädter, J. (2007). Entwicklungspsychologie der Lebensspanne: Leitvorstellungen und paradigmatische Orientierungen. In J. Brandtstädter \& U. Lindenberger (Hrsg.), Entwicklungspsychologie der Lebensspanne. Ein Lehrbuch (S. 34-66). Stuttgart: Kohlhammer.

Brett, J. F., \& VandeWalle, D. (1999). Goal orientation and goal content as predictors of performance in a training program. Journal of Applied Psychology, 84, 863-873.

Brophy, J. (2005). Goal theorists should move on from performance goals. Educational Psychologist, 40(3), 167-176. 
Buff, A. (1991). Schulische Selektion und Selbstkonzeptentwicklung. In R. Pekrun \& H. Fend (Hrsg.), Schule und Persönlichkeitsentwicklung: Ein Resümee der Längsschnittforschung (S. 100-114). Stuttgart: Enke.

Butler, R., \& Neuman, O. (1995). Effects of task and ego-achievement goals on help-seeking behaviors and attitudes. Journal of Educational Psychology, 87, 261-271.

Chiu, D., Beru, Y., Watley, E., Wubu, S., Simon, E., Kessinger, R., Rivera, A., et al. (2008). Influences of math tracking on seventh-grade students' self-beliefs and social comparisons. Journal of Educational Research, 102, 125-135.

Church, M. A., Elliot, A. J., \& Gable, S. (2001). Perceptions of classroom context, achievement goals, and achievement outcomes. Journal of Educational Psychology, 93, 4354.

Cialdini, R. B., Borden, R. J., Thorne, A., Walker, M. R., Freeman, S., \& Sloan, L. R. (1976). Basking in reflected glory: Three (football) field studies. Journal of Personality and Social Psychology, 34, 366-375.

Cohen, J. (1988). Statistical power analysis for the behavioral sciences $\left(2^{\text {nd }}\right.$ ed.). New Jersey: Lawrence Erlbaum.

Daniels, L. M., Haynes, T. L., Stupnisky, R. H., Perry, R. P., Newall, N. E., \& Pekrun, R. (2008). Individual differences in achievement goals: A longitudinal study of cognitive, emotional, and achievement outcomes. Contemporary Educational Psychology, 33, 584-608.

Daniels, Z. (2008). Entwicklung schulischer Interessen im Jugendalter. Münster: Waxmann.

Deci, E. L., \& Ryan, R. M. (1985). Intrinsic motivation and self-determination in human behavior. New York: Plenum Publishing Co.

Deci, E. L., Vallerand, R. J., Pelletier, L. G., \& Ryan, R. M. (1991). Motivation and education: The self-determination perspective. Educational Psychologist, 26, 325-346.

Diseth, A., \& Kobbeltvedt, T. (2010). A mediation analysis of achievement motives, goals, learning strategies, and academic achievement. British Journal of Educational Psychology, 80, 671-687.

Duda, L. J., \& Nicholls, J. G. (1992). Dimensions of achievement motivation in schoolwork and sport. Journal of Educational Psychology, 84, 290-299.

Duncan, T. E., Duncan, S. C., \& Strycker, L. A. (2006). An introduction to latent variable growth curve modeling ( $2^{\text {nd }}$ ed.). Mahwah: Erlbaum.

Dweck, C. S. (1986). Motivational processes affecting learning. American Psychologist, 41, 1040-1048. 
Dweck, C. S. (2000). Self-theories: Their role in motivation, personality, and development. Philadelphia, PA: Psychology Press.

Dweck, C. S., \& Leggett, E. L. (1988). A social- cognitive approach to motivation and personality. Psychological Review, 95, 256-273.

Eccles, J. S., Lord, S. E., Roeser, R. W., Barber, B. L., \& Jozefowicz, D. M. (1997). The association of school transitions in early adolescence with developmental trajectories through high school. In J.L. Maggs \& K. Hurrelmann (Eds.), Health risks and developmental transitions during adolescence (pp. 283-320). New York: Cambridge University Press.

Eccles, J. S., \& Midgley, C. (1989). Stage-environment fit: Developmentally appropriate classrooms for young adolescents. In Ames, C. \& Ames, R. (Eds.), Research on motivation in education (pp. 139-181). New York: Academic Press.

Eccles, J. S., \& Midgley, C. (1990). Changes in academic motivation and self-perceptions during early adolescence. In R. Montemayor, G. R. Adams, \& T.P. Gullotta (Eds.), Advances in adolescent development: From childhood to adolescence (pp. 134-155). Newbury Park: Sage.

Eccles, J. S., Midgley, C., Miller Buchanan, C., Reuman, D., Flanagan, C., \& Mac Iver, D. (1993). Development during adolescence: The impact of stage-environment fit on young adolescents' experiences in schools and in families. American Psychologist, 48, 90-101.

Elben, C. E., Lohaus, A., Ball, J., \& Klein-Heßling, J. (2003). Der Wechsel von der Grundschule zur weiterführenden Schule: Differentielle Effekte auf die psychische Anpassung. Psychologie in Erziehung und Unterricht, 50, 331-341.

Elliot, A. J. (1997). Integrating the „classic“ and „contemporary“ approaches to achievement motivation: A hierarchical model of approach and avoidance achievement motivation. In M.L. Maehr, \& P.R. Pintrich (Eds.), Advances in motivation and achievement, vol.10 (pp. 143-179). Greenwich, CT: JAI.

Elliot, A. J. (1999). Approach and avoidance motivation and achievement goals. Educational Psychologist, 34, 169-189.

Elliot, A. J., \& Church, M. A. (1997). A Hierarchical Model of Approach and Achievement Motivation. Journal of Personality and Social Psychology, 72, 218-232.

Elliot, A. J., \& Harackiewicz, J. M. (1996). Approach and avoidance goals and intrinsic motivation: A mediational analysis. Journal of Personality and Social Psychology, 70, 461-475. 
Elliot, A. J., \& McGregor, H. A. (1999). Test anxiety and the hierarchical model of approach and avoidance achievement motivation. Journal of Personality and Social Psychology, $76,628-644$.

Elliot, A. J., \& McGregor, H. (2001). A 2 x 2 achievement goal framework. Journal of Personality and Social Psychology, 80, 501-519.

Elliot, A. J., McGregor, H. A., \& Gable, S. (1999). Achievement goals, study strategies and exam performance: A mediational analysis. Journal of Educational Psychology, 91, 549-563.

Elliot, A. J., Murayama, K., \& Pekrun, R. (2011). A 3 X 2 achievement goal model. Journal of Educational Psychology, 103, 632-648.

Elliot, A. J., \& Sheldon, K. M. (1998). Avoidance goals and the personality-illness relationship. Journal of Personality and Social Psychology, 75, 1282-1299.

Elliot, A. J., \& Thrash, T. M. (2001). Achievement goals and the hierarchical model of achievement motivation. Educational Psychology Review, 13, 139-156.

Fenollar, P., Román, S., \& Cuestas, P. J. (2007). University students' academic performance: An integrative conceptual framework and empirical analysis. British Journal of Educational Psychology, 77, 873-891.

Elliot, A. J., Shell, M. M., Bouas Henry, K., \& Maier, M. A. (2005). Achievement goals, performance contingencies, and performance attainment: An experimental test. Journal of Educational Psychology, 97(4), 630-640.

Filipp, S. H. (1995). Ein allgemeines Modell für die Analyse kritischer Lebensereignisse. In S. H. Filipp, (Hrsg.), Kritische Lebensereignisse (S. 3-52). München: Psychologie Verlags Union.

Finney, S. J., Pieper, S. L., \& Barron, K. E. (2004). Examining the psychometric properties of the Achievement Goals Questionnaire in a general academic context. Educational and Psychological Measurement, 64, 365-382.

Finsterwald, M. (2006). Motivation und Schulübergang. Bedingungsfaktoren eines erfolgreichen Übergangs auf weiterführende Schulen. Berlin: Logos Verlag.

Finsterwald, M., Ziegler, A., \& Dresel, M. (2009). Individuelle Zielorientierung und wahrgenommene Klassenzielstruktur im Grundschulalter. Zeitschrift für Entwicklungspsychologie und Pädagogische Psychologie, 41(3), 143-152.

Graham, J. W., Cumsille, P. E., \& Elek-Fisk, E. (2003). Methods for handling missing data. In J. A. Schinka, W. F. Velicer, \& I. B. Weiner (Eds.), Handbook of psychology: Research methods in psychology (Vol. 2, pp. 87-114). New York: John Wiley \& Sons. 
Grant, H., \& Dweck, C. S. (2003). Clarifying achievement goals and their impact. Journal of Personality and Social Psychology, 85, 541-553.

Greene, B. A., \& Miller, R. B. (1996). Influences on achievement: Goals, perceived ability, and cognitive engagement. Contemporary Educational Psychology, 21, 181-192.

Greene, B. A., Miller, R. B., Crowson, H. M., Duke, B. L., \& Akey, K. L. (2004). Predicting high school students' cognitive engagement and achievement: Contributions of classroom perceptions and motivation. Contemporary Educational Psychology, 29, 462482.

Harackiewicz, J. M., Barron, K. E., Carter, S. M., Lehto, A. T., \& Elliot, A. J. (1997). Predictors and consequences of achievement goals in the college classroom: Maintaining interest and making the grade. Journal of Personality and Social Psychology, 73, 12841295.

Harackiewicz, J. M., Barron, K. E., \& Elliot, A. J. (1998). Rethinking achievement goals: When are they adaptive for college students and why? Educational Psychologist, 33, $1-21$.

Harackiewicz, J. M., Barron, K. E., Pintrich, P. R., Elliot, A. J., \& Thrash, T. M. (2002). Revision of achievement goal theory: Necessary and illuminating. Journal of Educational Psychology, 3, 638-645.

Harackiewicz, J. M., Barron, K. E., Tauer, J. M., Carter, S. M., \& Elliot, A. J. (2000). Shortterm and long-term consequences of achievement goals: Predicting interest and performance over time. Journal of Educational Psychology, 92, 316-330.

Harackiewicz, J. M., Barron, K. E., Tauer, J. M., \& Elliot, A. J. (2002). Prediction success in college: A longitudinal study of achievement goals and ability measures as predictors of interest and performance from freshman year through graduation. Journal of Educational Psychology, 94, 562-575.

Harackiewicz, J. M., Durik, A. M., Barron, K. E., Linnenbrink, E. A., \& Tauer, J. M. (2008). The Role of achievement goals in the development of interest: Reciprocal relations between achievement goals, interest, and performance. Journal of Educational Psychology, 100, 105-122.

Harazd, B., \& Schürer, S. (2006). Veränderungen der Schulfreude von der Grundschule zur weiterführenden Schule. In A. Schründer-Lenzen (Hrsg.), Risikofaktoren kindlicher Entwicklung. Migration, leistungsbezogene Emotionen und der Übergang von der Grundschulzeit zu den weiterführenden Schulen (S. 208-222). Wiesbaden: VS Verlag. 
Hasselhorn, M., \& Labuhn, A. (2008). Metakognition und selbstreguliertes Lernen. In Schneider, W. \& Hasselhorn, M. (Hrsg.), Handbuch Pädagogische Psychologie (S. 28-37). Göttingen: Hogrefe.

Heller, K. A., \& Perleth, C. (2000). Kognitiver Fähigkeitstest für 4. bis 12. Klassen, Revision. Göttingen: Beltz.

Helmke, A. (1992). Selbstvertrauen und schulische Leistungen. Göttingen: Hogrefe.

Helmke, A. (2009). Unterrichtsqualität und Lehrerprofessionalität: Diagnose, Evaluation und Verbesserung des Unterrichts. Seelze: Klett Kallmeyer.

Hodapp, V., Laux, L., \& Spielberger, C. D. (1982). Theorie und Messung der emotionalen und kognitiven Komponente der Prüfungsangst. Zeitschrift für Differentielle und Diagnostische Psychologie, 3, 169-184.

Honaker, J., King, G., \& Blackwell, M. (2006). Amelia Software Web Site. Available online at hppt://gking.harvard.edu/amelia.

Hu, L., \& Bentler, P. M. (1999). Cutoff criteria for fit indexes in covariance structure analysis: conventional criteria versus new alternatives. Structural Equation Modeling, 6(1), $1-55$.

Hübner, S., Nückles, M., \& Renkl, A. (2010). Writing learning journals: Instructional support to overcome learning-strategy deficits. Learning and Instruction, 20, 18-29.

Hulleman, C. S., Durik, A. M., Schweigert, S. A., \& Harackiewicz, J. M. (2008). Task values, achievement goals, and interest: An integrative analysis. Journal of Educational Psychology, 100, 398-416.

Hulleman, C. S., Schrager, S. M., Bodmann, S. M., \& Harackiewicz, J. M. (2010). A metaanalytic review of achievement goal measures: Different labels for the same constructs or different constructs with similar labels? Psychological Bulletin, 136, 422-449.

Ireson, J., \& Hallam, S. (2001). Ability grouping in education. London: Sage.

Jonkmann, K., Maaz, K., McElvany, N., \& Baumert, J. (2010). Die Elternentscheidung beim Übergang in die Sekundarstufe I - Eine theoretische Adaption und empirische Überprüfung des Erwartungs-Wert-Modells. In K. Maaz, J. Baumert, C. Gresch \& N. McElvany (Hrsg.), Der Übergang von der Grundschule in die weiterführende Schule Leistungsgerechtigkeit und regionale, soziale und ethnisch-kulturelle Disparitäten. (S. 253-282). Bonn, Berlin: BMBF.

Juvonen, J., Le, V., Kaganoff, T., Augustine, C., \& Constant, L. (2004). Focus on the wonder years: Challenges facing the American middle school. Santa Monica, CA: RAND Corporation. 
Kaplan, A., \& Maehr, M. (1999). Achievement goals and student well-being. Contemporary Educational Psychology, 24, 330-358.

Karabenick, S. A. (2004). Perceived achievement goal structure and college student help seeking. Journal of Educational Psychology, 96, 569-581.

Kelly, J. G., Ryan, A. M., Altman, B. E., \& Stelzner, S. P. (1993). Understanding and changing social systems: An ecological view. In E. Seidman \& J. Rappaport (Eds.), Handbook of community psychology. New York: Plenum Press.

Kenny, D. A. (1975). Cross-lagged panel correlation: A test for spuriousness. Psychological Bulletin, 82, 887-903.

Köller, O. (1998). Zielorientierungen und schulisches Lernen. Berlin: Waxmann.

Köller, O. (2004). Konsequenzen von Leistungsgruppierungen. Münster: Waxmann.

Köller, O. (2005). Gesamtschule- Erweiterung statt Alternative. In K. S. Cortina, J. Baumert, K. U. Leschinsky, U. Mayer \& L. Trommer (Hrsg.), Das Bildungswesen in der Bundesrepublik Deutschland (2. Aufl., S. 458-486). Reinbek bei Hamburg: Rewohlt- Taschenbuch-Verlag.

Köller, O., \& Baumert, J. (1998). Ein deutsches Instrument zur Erfassung von Zielorientierungen bei Schülerinnen und Schülern. Diagnostica, 44, 173-181.

Köller, O., Schnabel, K. U., \& Baumert, J. (2000). Der Einfluß der Leistungsstärke von Schulen auf das fachspezifische Selbstkonzept der Begabung und das Interesse. Zeitschrift für Entwicklungspsychologie und Pädagogische Psychologie, 32, 70-80.

Köller, O., Trautwein, U., Lüdtke, O., \& Baumert, J. (2006). Zum Zusammenspiel von schulischer Leistung, Selbstkonzept und Interesse in der gymnasialen Oberstufe. Zeitschrift für Pädagogische Psychologie, 20, 27-39.

Kramer, R.-T., Helsper, W., Thiersch, S., \& Ziems, C. (2009). Selektion und Schulkarriere. Kindliche Orientierungsrahmen beim Übergang in die Sekundarstufe I. Wiesbaden: VS Verlag für Sozialwissenschaften.

Kropf, M., Gresch, C., \& Maaz, K. (2010). Überblick über die rechtlichen Regelungen des Übergangs in den beteiligten Ländern. In K. Maaz, J. Baumert, C. Gresch \& N. McElvany (Hrsg.), Der Übergang von der Grundschule in die weiterführende Schule Leistungsgerechtigkeit und regionale, soziale und ethnisch-kulturelle Disparitäten. (S. 399-429). Bonn, Berlin: BMBF.

Kunter, M., \& Baumert, J. (2006). Linking TIMSS to research on learning and instruction: A re-analysis of the German TIMSS and TIMSS video data. In S. J. Howie, \& T. Plomp (Eds.), Contexts of learning mathematics and science: Lessons learned from TIMSS (pp. 335-351). London: Routledge. 
Kunter, M., Schümer, G., Artelt, C., Baumert, J., Klieme, E., Neubrand, M., Prenzel, M., et al. (2002). PISA 2000- Dokumentation der Erhebungsinstrumente. Berlin: Max-PlanckInstitut für Bildungsforschung.

Kurtz, T., Watermann, R., Klingebiel, F., \& Szczesny, M. (2010). Das emotionale Erleben des bevorstehenden Grundschulübergangs und die Rolle der elterlichen Unterstützung. In K. Maaz, J. Baumert, C. Gresch \& N. McElvany (Hrsg.), Der Übergang von der Grundschule in die weiterführende Schule Leistungsgerechtigkeit und regionale, soziale und ethnisch-kulturelle Disparitäten. Bonn, Berlin: BMBF.

Lau, S., \& Nie, Y. (2008). Interplay between personal goals and classroom goal structures in predicting student outcomes: A multilevel analysis of person-context interactions. Journal of Educational Psychology, 100, 15-29.

Lee, F. K., Sheldon, K. M., \& Turban, D. B. (2003). Personality and the goal-striving process: The influence of achievement goal patterns, goal level and mental focus on performance and enjoyment. Journal of Applied Psychology, 88, 256-265.

Lemos, M. S. (1999). Students' goals and self-regulation in the classroom. International Journal of Educational Research, 31, 471-485.

Leschinsky, A. (2005). Der institutionelle Rahmen des Bildungswesens. In K. S. Cortina, J. Baumert, K. U. Leschinsky, U. Mayer, \& L. Trommer (Hrsg.), Das Bildungswesen in der Bundesrepublik Deutschland (2. Aufl., S. 148-213). Reinbek bei Hamburg:

Rewohlt- Taschenbuch-Verlag.

Liegmann, A., \& Lumer, B. (2004). Zukunft oder Zumutung? Zum Übergang von der Grundschule zur Hauptschule. In E. Schumacher (Hrsg.), Übergänge in Bildung und Ausbildung-Gesellschaftliche, subjektive und pädagogische Relevanzen (S. 103-124). Bad Heilbronn/ OBB.: Klinkhardt.

Linnenbrink, E. A. (2005). The dilemma of performance-approach goals: The use of multiple goal contexts to promote students' motivation and learning. Journal of Educational Psychology, 97, 197-213.

Linnenbrink, E. A., \& Pintrich, P. R. (2002). Achievement goal theory and affect: An asymmetrical bidirectional model. Educational Psychologist, 37, 69-78.

Linnenbrink, L., \& Pintrich, P. R. (2000). Multiple pathways to learning and achievement: The role of goal orientation in fostering adaptive motivation, affect and cognition. In C. Sansone, \& J.M. Harackiewicz, (Eds.), Intrinsic and extrinsic motivation: The search for optimal motivation and performance (pp. 196-227). New York: Academic. 
Linnenbrink-Garcia, L., Tyson, D. F., \& Patall, E. A. (2008). When are achievement goal orientations beneficial for academic achievement? A closer look at main effects and moderating factors. International Review of Social Psychology, 21, 19-70.

Litman, J. A., \& Spielberger, C. D. (2003). Measuring epistemic curiosity and its diversive and specific components. Journal of Personality Assessment, 80, 75-86.

Little, R. J. A., \& Rubin, D. B. (2002). Statistical analysis with missing data (2 ${ }^{\text {nd }}$ ed.). New York: John Wiley.

Maaz, K., Baumert, J., Gresch, C., \& McElvany, N. (2010). Der Übergang von der Grundschule in die weiterführende Schule. Leistungsgerechtigkeit und regionale, soziale und ethnisch-kulturelle Disparitäten. Bonn, Berlin: BMBF.

Maaz, K., Neumann, M., Trautwein, U., Wendt, W., Lehmann, R., \& Baumert, J. (2008). Der Übergang von der Grundschule in die weiterführende Schule. Die Rolle von Schülerund Klassenmerkmalen beim Einschätzen der individuellen Lernkompetenz durch die Lehrkräfte. Schweizerische Zeitschrift für Bildungswissenschaften, 30, 519-548.

Maaz, K., Trautwein, U., Lüdtke, O., \& Baumert, J. (2008). Educational transitions and differential learning environments: How explicit between-school tracking contributes to social inequality in educational outcomes. Child Development Perspectives, 2, 99-106.

Maehr, M. L., \& Midgley, C. (1991). Enhancing student motivation: A schoolwide approach. Educational Psychologist, 26, 399-427.

Marsh, H. W. (1984). Self-concept: The application of a frame of reference model to explain paradoxical results. Australian Journal of Education, 28, 165-181.

Marsh, H. W. (1986). Verbal and math self-concept: An internal/external frame of reference model. American Educational Research Journal, 23, 129-149.

Marsh, H. W. (1987). The big-fish-little-pond effect on academic self-concept. Journal of Educational Psychology, 79, 280-295.

Marsh, H. W. (1990). A multidimensional hierarchical self-concept: Theoretical and empirical justification. Educational Psychology Review, 2, 77-171.

Marsh, H. W., \& Craven, R. G. (1997). Academic self-concept: Beyond the dustbowl. In G. D. Phye (Ed.), Handbook of classroom assessment (pp. 131-198). San Diego, CA: Academic Press.

Marsh, H. W., Craven, R. G., \& Debus, R. (1991). Self-concepts of young children 5 to 8 years of age: Measurement and multidimensional structure. Journal of Educational Psychology, 83, 377-392. 
Marsh, H. W., \& Hau, K. T. (2003). Big fish little pond effect on academic self-concept: A cross-cultural (26 country) test of the negative effects of academically selective schools. American Psychologist, 58, 364-376.

Marsh, H. W., \& O’Mara, A. J. (2010). Long-term total negative effects of school-average ability on diverse educational outcomes. Zeitschrift für Pädagogische Psychologie, 24, $51-72$.

Marsh, H. W., Köller, O., \& Baumert, J. (2001). Reunification of East and West German school systems: Longitudinal multilevel modelling study of the big-fish-little-pond effect on academic self-concept. American Educational Research Journal, 38, 321-350.

Marsh, H. W., Kong, C. K., \& Hau, K. (2000). Longitudinal multilevel models of the big-fishlittle-pond effect on academic self-concept: Counterbalancing contrast and reflectedglory effects in Hong Kong schools. Journal of Personality and Social Psychology, $78(2), 337-349$.

Marsh, H. W., Trautwein, U., Lüdtke, O., Köller, O., \& Baumert, J. (2005). Academic selfconcept, interest, grades and standardized test scores: Reciprocal effects models of causal ordering. Child Development, 76, 397-416.

Marsh, H. W., \& Yeung, A. S. (1997). Causal effects of academic self-concept on academic achievement: Structural equation models of longitudinal data. Journal of Educational Psychology, 89, 41-54.

McKinney, A. P. (2003). Goal Orientation: A Test of Competing Models. Virginia Polytechnic Institute and State University.

Meece, J. L., Blumenfeld, P. C., \& Hoyle, R. H. (1988). Students`́ goal orientations and cognitive engagement in classroom activities. Journal of Educational Psychology, 80, 514-523.

Meredith, W., \& Tisak, J. (1990). Latent curve analysis. Psychometrika, 55, 107-122.

Middleton, M. J., Kaplan, A., \& Midgley, C. (2004). The change in middle school students' achievement goals in mathematics over time. Social Psychology of Education, 7, 289311.

Middleton, M. J., \& Midgley, C. (1997). Avoiding the demonstration of lack of ability: An under explored aspect of goal theory. Journal of Educational Psychology, 89, 710718.

Midgley, C., Anderman, E., \& Hicks, L. (1995). Differences between elementary and middle school teachers and students: A goal theory approach. Journal of Early Adolescence, $15,90-113$. 
Midgley, C., Feldlaufer, H., \& Eccles, J. S. (1989). Change in teacher efficacy and student self-and task-related beliefs in mathematics during the transition to junior high school. Journal of Educational Psychology, 81(2), 247-258.

Midgley, C., Kaplan, A., \& Middleton, M. (2001). Performance-approach goals: Good for what, for whom, under what circumstances, and at what cost? Journal of Educational Psychology, 93(1), 77-86.

Midgley, C., Kaplan, A., Middleton, M., Maehr, M. L., Urdan, T., Anderman, L. H., Anderman, E., et al. (1998). The development and validation of scales assessing students' achievement goal orientations. Contemporary Educational Psychology, 23, 113-131.

Midgley, C., \& Maehr, M. L. (1993). Patterns of Adaptive Learning Survey. Ann Arbor, MI: University of Michigan.

Midgley, C., Maehr, M. L., Hruda, L. Z., Anderman, E., Anderman, L., Freeman, K. E., Gheen, M., et al. (2000). Manual for the patterns of adaptive learning scales. Michigan: University of Michigan.

Midgley, C., \& Urdan, T. (1995). Predictors of middle school students' use of selfhandicapping strategies. Journal of Early Adolescence, 26, 389-411.

Midgley, C., \& Urdan, T. (2001). Academic self-handicapping and achievement goals: A further examination. Contemporary Educational Psychology, 26, 61-75.

Miller, R. B., Behrens, J. T., Greene, B. A., \& Newman, D. (1993). Goals and perceived ability: Impact on student valuing, self-regulation, and persistence. Contemporary Educational Psychology, 18, 2-14.

Moosbrugger, H., \& Kelava, A. (2008). Testtheorie und Fragebogenkonstruktion. Heidelberg: Springer Medizin Verlag.

Mullis, I. V. S., Martin, M. O., Ruddock, G. J., O’Sullivan, C. Y., Arora, A., \& Erberber, E. (2005). TIMSS 2007 assessment frameworks. Chestnut Hill, MA: TIMSS \& PIRLS International Study Center, Boston College.

Muthén, L. K., \& Muthén, B. O. (1998-2010). Mplus 6.1 [Computer software]. Los Angeles, CA: Muthén \& Muthén. http://statmodel.com.

Muthén, L. K., \& Muthén, B. O. (1998-2011). Mplus 6.11 [Computer software]. Los Angeles, CA: Muthén \& Muthén. http://statmodel.com.

Nauck, J., \& Otte, R. (1980). Diagnostischer Test Deutsch (DTD). Göttingen: Hogrefe.

Nicholls, J. G. (1978). The development of the concepts of effort and ability, perception of academic attainment, and the understanding that difficult tasks require more ability. Child Development, 49, 800-814. 
Nicholls, J. G. (1984). Achievement motivation: Conceptions of ability, subjective experience, task choice and performance. Psychological Review, 91, 328-346.

Nicholls, J. G. (1989). The competitive ethos and democratic education. Cambridge, MA: Harvard University Press.

Nicholls, J. G. (1990). What is ability and why are we mindful of it? A developmental perspective. In R. Sternberg \& J. Kolligian (Eds.), Competence considered (pp. 11-40). New Haven, CT: Yale University Press.

Nicholls, J. G. (1992). Students as educational theorists. In D. H. Schunk, \& J. L. Meece, (Eds.), Students perceptions in the classroom (pp. 267-287). Hillsdale, NJ: Erlbaum.

Nicholls, J. G., Patashnick, M., \& Nolen, S. (1985). Adolescents' theories of education. Journal of Educational Psychology, 77, 683-692.

Nolen, S. B. (1988). Reasons for studying: Motivational orientations and study strategies. Cognition and Instruction, 5, 269-287.

van Ophuysen, S. (2006). Zur Problematik der Schulformempfehlungen nach der Grundschulzeit und ihrer prognostischen Qualität. In W. Bos et al. (Hrsg.), Jahrbuch der Schulentwicklung (Bd. 14, S. 49-81). Weinheim/ München: Juventa.

van Ophuysen, S. (2008). Zur Veränderung der Schulfreude von Klasse 4 bis 7. Eine Längsschnittanalyse schulformspezifischer Effekte von Ferien und Grundschulübergang. Zeitschrift für Pädagogische Psychologie, 22, 293-306.

Pajares, F., Britner, S., \& Valiante, G. (2000). Relation between achievement goals and selfbeliefs of middle school students in writing and science. Contemporary Educational Psychology, 25, 406-422.

Pajares, F., \& Valiante, G. (2001). Gender differences in writing motivation and achievement of middle school students: A function of gender orientation? Contemporary Educational Psychology, 26(3), 366-381.

Paulick, I., Watermann, R., \& Nückles, M. (2011a). Achievement goals and school achievement: The transition to different school tracks in secondary school. Manuscript submitted for publication.

Paulick, I., Watermann, R., \& Nückles, M. (2011b). Effects of tracking on students'selfconcept, intrinsic motivation and achievement goals during the transition to secondary school. Manuscript in preparation.

Paulick, I., Watermann, R., \& Nückles, M. (2011c). Zielorientierungen und schulisches Lernen am Grundschulübergang. Manuscript submitted for publication. 
Pekrun, R., Elliot, A. J., \& Maier, M. A. (2006). Achievement goals and discrete achievement emotions: A theoretical model and prospective test. Journal of Educational Psychology, 98, 583-597.

Pekrun, R., Elliot, A. J., \& Maier, M. A. (2009). Achievement goals and achievement emotions: Testing a model of their joint relations with academic performance. Journal of Educational Psychology, 101, 115-135.

Pekrun, R., Goetz, T., Titz, W., \& Perry, P. P. (2002). Academic emotions in students' selfregulated learning and achievement: A program of qualitative and quantitative research. Educational Psychologist, 37, 91-105.

Pintrich, P. R. (1999). The role of motivation in promoting and sustaining self-regulated learning. International Journal of Educational Research, 31, 459-470.

Pintrich, P. R. (2000a). An achievement goal theory perspective on issues in motivation terminology, theory, and reserach. Contemporary Educational Psychology, 25(1), 92 104.

Pintrich, P. R. (2000b). The role of goal orientation in self-regulated learning. In M. Boekaerts, P.R. Pintrich, \& M. Zeidner (Eds.), Handbook of self-regulation (pp. 451-502). San Diego, CA: Academic.

Pintrich, P. R., \& De Groot, E. V. (1990). Motivational and self-regulated learning components of classroom academic performance. Journal of Educational Psychology, 82, 3340.

Preckel, F., \& Brüll, M. (2010). The benefit of being a big fish in a big pond: Contrast and assimilation effects on academic self-concept. Learning and Individual Differences, $20,522-531$.

Rawsthorne, L. J., \& Elliot, A. J. (1999). Achievement goals and intrinsic motivation: A meta-analytic review. Personality and Social Psychology Review, 3, 326-344.

Roeser, R. W., Midgley, C., \& Urdan, T. C. (1996). Perceptions of the school psychological environment and early adolescents' psychological and behavioral functioning in school: The mediating role of goals and belonging. Journal of Educational Psychology, 88(3), 408-422.

Ross, M. E., Shannon, D. M., Salisbury-Glennon, J. D., \& Guarino, A. (2002). The pattern of adaptive learning survey: A comparison across grade levels. Educational and Psychological Measurement, 62, 483-497.

Rubin, R. B. (1987). Multiple imputation for nonresponse in surveys. New York: Wiley. 
Ruble, D. N., \& Frey, K. S. (1991). Changing patterns of comparative behavior as skills are acquired: A functional model of self-evaluation. In J. Suls, \& T. A. Wills, (Eds.), Social comparison: Contemporary theory and research (pp. 79-113). Hillsdale, NJ: Erlbaum.

Ryan, A. M., Patrick, H., \& Shim, S. (2005). Differential profiles of students identified by their teacher as having avoidant, appropriate, or dependent help-seeking tendencies in the classroom. Journal of Educational Psychology, 97, 275-285.

Ryan, A. M., Pintrich, P. R., \& Midgley, C. (2001). Avoiding seeking help in the classroom: Who and why? Educational Psychology Review, 13, 93-114.

Schiefele, U. (2009). Motivation. In E. Wild \& J. Möller (Hrsg.), Pädagogische Psychologie. Heidelberg: Springer.

Schöne, C. (2007). Zielorientierung und Bezugsnormpräferenzen in Lern- und Leistungssituationen. Unveröffentlichte Dissertation, Justus-Liebig-Universität Gießen.

Schunk, D. H., Pintrich, P. R., \& Meece, J. L. (2008). Motivation in education: Theory, research, and applications ( $3^{\text {rd }}$ ed.). Upper Saddle River, New Jersey: Pearson/Merrill Prectice Hall.

Schwarzer, R., Lange, B., \& Jerusalem, M. (1982). Selbstkonzeptentwicklung nach einem Bezugsgruppenwechsel. Zeitschrift für Entwicklungspsychologie und Pädagogische Psychologie, 14, 125-140.

Schwinger, M., \& Wild, E. (2006). Die Entwicklung von Zielorientierungen im Fach Mathematik von der 3. bis 5. Jahrgangsstufe. Zeitschrift für Pädagogische Psychologie, 20(4), 269-278.

Senko, C., \& Harackiewicz, J. M. (2005). Regulation of achievement goals: The role of competence feedback. Journal of Educational Psychology, 97, 320-336.

Shim, S. S., Ryan, A. M., \& Anderson, C. J. (2008). Achievement goals and achievement during early adolescence: Examining time-varying predictors and outcome variables in growth-curve analysis. Journal of Educational Psychology, 100(3), 655-671.

Sideridis, G. D. (2005). Goal orientation, academic achievement, and depression: Evidence in favor of a revised goal theory framework. Journal of Educational Psychology, 97, 366-375.

Sirsch, U. (2000). Probleme beim Schulwechsel. Die subjektive Bedeutung des bevorstehenden Wechsels von der Grundschule in die weiterführenden Schule. Münster: Waxmann. 
Sirsch, U. (2003). The impending transition from primary to secondary school: Challenge or threat? International Journal of Behavioral Development, 27, 385-395.

Skaalvik, E. M. (1997). Self-enhancing and self-defeating ego orientation: Relations with task and avoidance orientation, achievement, self-perceptions, and anxiety. Journal of Educational Psychology, 89, 71-81.

Skaalvik, E. M., \& Skaalvik, S. (2002). Internal and external frames of reference for academic self-concept. 37, 233-244.

Sparfeldt, J. R., Buch, S. R., Wirthwein, L., \& Rost, D. H. (2007). Zielorientierungen: Zur Relevanz der Schulfächer. Zeitschrift für Entwicklungspsychologie und Pädagogische Psychologie, 39, 165-176.

Spinath, B., \& Schöne, C. (2003). Ziele als Bedingungen von Motivation am Beispiel der Skalen zur Erfassung der Lern- und Leistungsmotivation (SELLMO). In J.

Stiensmeier-Pelster \& F. Rheinberg (Hrsg.), Diagnostik von Motivation und Selbstkonzept (Tests und Trends, Jahrbuch der pädagogisch-psychologischen Diagnostik. N. F., Band 2) (S. 29-40). Göttingen: Hogrefe.

Spinath, B., Stiensmeier-Pelster, J., Schöne, C., \& Dickhäuser, O. (2002). Die Skalen zur Erfassung der Lern- und Leistungsmotivation (SELLMO). Göttingen: Hogrefe.

Ständige Konferenz der Kultusminister der Länder in der Bundesrepublik Deutschland (2010). Das Bildungswesen in der Bundesrepublik Deutschland 2009. Darstellung der Kompetenzen, Strukturen und bildungspolitischen Entwicklungen für den Informationsaustausch in Europa. Bonn, KMK.

Stipek, D., \& Gralinski, J. H. (1996). Children's beliefs about intelligence and school performance. Journal of Educational Psychology, 88, 397-407.

Südkamp, A., \& Möller, J. (2009). Referenzgruppeneffekt im Simulierten Klassenraum. Direkte und indirekte Einschätzungen von Schülerleistungen. Zeitschrift für Pädagogische Psychologie, 23(3-4), 161-174.

Tanaka, A., \& Yamauchi, H. (2001). A model for achievement motives, goal orientations, intrinsic interest, and academic achievement. Psychological Reports, 88, 123-135.

Tippelt, R. (2000). Der schwierige Übergang vom Bildungs- in das BeschäftigungssystemNotwendigkeit und Möglichkeit zur Weiterbildung. In C. Harteis, H. Heid \& S. Kraft (Hrsg.), Kompendium Weiterbildung. Aspekte und Perspektiven betrieblicher Personal- und Organisationsentwicklung (S. 69-79). Opladen: Leske+Budrich. 
Tippelt, R. (2004). Geleitwort. In E. Schumacher (Hrsg.), Übergänge in Bildung und Ausbildung-Gesellschaftliche, subjektive und pädagogische Relevanzen (S. 7-17). Bad Heilbronn/ OBB.: Klinkhardt.

Trautwein, U., Köller, O., Lüdtke, O., \& Baumert, J. (2005). Student tracking and the powerful effects of opt-in courses on self-concept: Reflected-glory effects do exist after all. In H. W. Marsh, R. Craven \& D. M. McInerney (Eds.), New frontiers for self research (pp. 307-327). Greenwich, CT: IAP.

Trautwein, U., \& Lüdtke, O. (2005). The big fish little pond effect: Future research questions and educational implications. Zeitschrift für Pädagogische Psychologie, 19, 137-140.

Trautwein, U., Lüdtke, O., Köller, O., \& Baumert, J. (2006). Self-esteem, academic selfconcept, and achievement: How the learning environment moderates the dynamics of self-concept. Journal of Personality and Social Psychology, 90, 334-349.

Trautwein, U., Lüdtke, O., Marsh, H. W., Köller, O., \& Baumert, J. (2006). Tracking, grading, and student motivation: Using group composition and status to predict selfconcept and interest in ninth-grade mathematics. Journal of Educational Psychology, 98, 788-806.

Trautwein, U., Lüdtke, O., Marsh, H. W., \& Nagy, G. (2009). Within-school social comparison: How students perceive the standing of their class predicts academic self-concept. Journal of Educational Psychology, 101, 853-866.

Urdan, T. (2004). Predictors of academic self-handicapping and achievement: Examining achievement goals, classroom goal structures, and culture. Journal of Educational Psychology, 96, 251-264.

Utman, C. H. (1997). Performance effects of motivational state: A meta-analysis. Personality and Social Psychology Review, 1, 170-182.

Valtin, R., \& Wagner, C. (2004). Der Übergang in die Sekundarstufe I: Psychische Kosten der externen Leistungsdifferenzierung. Psychologie in Erziehung und Unterricht, 51, 5268.

Vansteenkiste, M., Simons, J., Lens, W., Soenens, B., Matos, L., \& Lacante, M. (2004). Less is sometimes more: Goal content matters. Journal of Educational Psychology, 96, 755764.

Watermann, R., Klingebiel, F., \& Kurtz, T. (2010). Die motivationale Bewältigung des Grundschulübergangs aus Schüler- und Elternsicht. In K. Maaz, J. Baumert, C. Gresch \& N. McElvany (Hrsg.), Der Übergang von der Grundschule in die weiterführende 
Schule Leistungsgerechtigkeit und regionale, soziale und ethnisch-kulturelle Disparitäten. (S. 355-383). Bonn, Berlin: BMBF.

Weinert, F. E., \& Stefanek, J. (1997). Entwicklung vor, während und nach der Grundschulzeit. Ergebnisse aus dem SCHOLASTIK-Projekt. In F. E. Weinert \& A. Helmke (Hrsg.), Entwicklung im Grundschulalter (S. 423-451). Weinheim: Beltz, PsychologieVerlagsUnion.

Weitzel, C. (2004). Bruch, Brücke, Chance- oder nur ein nutzloses historisches Relikt? Übergänge nach dem 4. Schuljahr. In L. Denner \& E. Schuhmacher (Hrsg.), Übergänge im Elementar- und Primarbereich reflektieren und gestalten. Beiträge zu einer grundlegenden Bildung (S. 106-119). Bad Heilbronn/ OBB.: Klinkhardt.

Wentzel, K. R. (1993). Motivation and achievement in early adolescence: The role of multiple classroom goals. Journal of Early Adolescence, 13, 4-20.

Wigfield, A., \& Eccles, J. S. (1992). The development of achievement task values: A theoretical analysis. Developmental Review, 12, 265-310.

Wigfield, A., Eccles, J. S., MacIver, D., Reuman, D. A., \& Midgley, C. (1991). Transitions during early adolescence: Changes in children's domain- specific self-perceptions and general self-esteem across the transition to Junior High School. Developmental Psychology, 27(4), 552-565.

Wild, E., \& Möller, J. (2009). Pädagogische Psychologie. Berlin: Springer.

Wolters, C. A. (2004). Advancing achievement goal theory: Using goal structures and goal orientations to predict students' motivation, cognition, and achievement. Journal of Educational Psychology, 2, 236-250.

Wolters, C., Yu, S., \& Pintrich, P. R. (1996). The relation between goal orientation and students' motivational beliefs and self-regulated learning. Learning and Individual Differences, 8, 211-238.

Wu, M. L., Adams, R. J., \& Wilson, M. R. (1998). ACER ConQuest: Generalized item response modelling software. Melbourne: Acer.

Yeo, G., Loft, S., Xiao, T., \& Kiewitz, C. (2009). Goal orientations and performance: Differential relationships across levels of analysis and as a function of task demands. Journal of Applied Psychology, 3, 710-726.

Yu, C. (2002). Evaluating cutoff criteria of model fit indices for latent variable models with binary and continuous outcomes. University of California, Los Angeles, CA.

Zeinz, H., \& Köller, O. (2006). Noten, soziale Vergleiche und Selbstkonzepte in der Grundschule. In A. Schründer-Lenzen (Hrsg.), Risikofaktoren kindlicher Entwicklung. Mig- 
ration, Leistungsangst und Schulübergang (S. 177-190). Wiesbaden: VS Verlag für Sozialwissenschaften.

Zimmerman, B. J. (1998). Academic studying and the development of personal skill: A selfregulatory perspective. Educational Psychologist, 33, 73-86.

Zimmerman, B. J., \& Campillo, M. (2003). Motivating self-regulated problem solvers. In J.E Davidson \& R. Sternberg (Eds.), The psychology of problem solving (pp. 233-263). New York: Cambridge University Press. 
Anhang A

\section{Itemübersicht und Ergebnisse der konfirmatorischen Faktorenanalyse (standardisierte Faktorladungen)}

Items

Faktor-

ladungen

Es ist mir wichtig,...

Lernziele

...so viel wie möglich zu lernen.

...viele neue Fähigkeiten zu erwerben.

...dass ich den Unterrichtsstoff gründlich verstehe.

...dass ich meine Fähigkeiten verbessere.

Annäherungsleistungsziele

...dass ich mehr weiß als die anderen.

...dass der Lehrer mich für einen der besten Schüler hält.

...dass ich als einziger die richtige Antwort weiß.

...dass ich mehr Aufgaben richtig habe als meine Klassenkameraden.

\section{Vermeidungsleistungsziele}

...mich vor den anderen Schülern nicht zu blamieren.

...dass mich die anderen nicht für blöd halten.

Anmerkungen. Alle Koeffizienten sind statistisch signifikant $(p<.001)$. 
Anhang B

\section{Itemsübersicht Studie 1: Intrinsische Motivation, akademisches Selbstkon- zept und Leistungsangst}

\section{Items}

\section{Intrinsische Motivation (Berlyne, 1978; Litman \& Spielberger, 2003)}

Wie geht es dir beim Lernen innerhalb und außerhalb der Schule?

Lernen macht mir Spaß.

Ich bin immer ganz neugierig, wenn ich Neues lernen kann.

Neues zu lernen, ist oft aufregend.

Wenn ich etwas noch nicht richtig verstanden habe, nutze ich auch die Zeit nach der Schule.

Etwas Neues zu verstehen, gehört für mich zu den wichtigsten Dingen.

Manchmal bin ich richtig gierig, weiter lernen zu können.

Wenn ich gerade richtig bei der Sache bin, mag ich gar nicht aufhören.

Manchmal bin ich beim Lernen völlig versunken.

\section{Akademisches Selbstkonzept (Marsh, 1990; vgl. Kunter et al., 2002)}

Wie geht es dir beim Lernen in den Fächern?

In den meisten Schulfächern lerne ich schnell.

In den meisten Schulfächern schneide ich in Klassenarbeiten/Schularbeiten gut ab. Ich bin in den meisten Schulfächern gut.

\section{Leistungsangst (emotionality: vgl. Hodapp et al., 1982; worry: vgl. Helmke, 1992)}

Wie geht es dir bei den Klassenarbeiten/Schularbeiten? Bitte denk einmal an die letzten Klassenarbeiten/Schularbeiten in Deutsch oder Mathematik. Kam Folgendes bei dir vor?

Ich war am ganzen Körper verkrampft. (emotionality)

Ich war so nervös, dass ich kaum noch arbeiten konnte. (emotionality)

Das Herz schlug mir bis zum Hals. (emotionality)

Ich fühlte mich verwirrt und durcheinander. (emotionality)

Ich dachte daran, was meine Eltern von mir erwarten. (worry)

Ich dachte daran, was die anderen tun. (worry)

Ich dachte daran, was ich alles nicht kann. (worry)

Meine Gedanken schweiften von der Arbeit ab. (worry)

Ich dachte an Sachen, die mit der Arbeit gar nichts zu tun hatten. (worry) 


\section{Übersicht der eingesetzten SELLMO-Items}

\section{Items}

In der Schule geht es mir darum,...

Lernziele

... etwas Interessantes zu lernen.

... später knifflige Probleme lösen zu können.

... dass das Gelernte für mich Sinn ergibt.

... neue Ideen zu bekommen.

... so viel wie möglich zu lernen.

... komplizierte Inhalte zu verstehen.

... zum Nachdenken angeregt zu werden.

... ein tiefes Verständnis für die Inhalte zu erwerben.

\section{Annäherungsleistungsziele}

... Arbeiten besser zu schaffen als andere.

... bessere Noten oder Beurteilungen zu bekommen als andere.

... zu zeigen, dass ich bei einer Sache gut bin.

... das, was ich kann und weiß, auch zu zeigen

... dass andere denken, dass ich klug bin.

... dass die anderen merken, dass ich in Tests und Prüfungen gut abschneide.

... zu zeigen, dass ich die Inhalte beherrsche.

\section{Vermeidungsleistungsziele}

... keine falschen Antworten auf Fragen der Lehrer zu geben.

... dass niemand merkt, wenn ich etwas nicht verstehe.

... nicht zu zeigen, wenn mir eine Aufgabe schwerer fällt als den anderen.

... zu verbergen, wenn ich weniger weiß als andere.

... mich nicht zu blamieren (z.B. durch falsche Ergebnisse oder dumme Fragen).

... nicht zu zeigen, falls ich weniger schlau bin als andere.

... nicht durch dumme Fragen aufzufallen.

... dass andere Schüler nicht denken, ich sei dumm. 